\title{
Erbabgeordnete in Japan - Entstehung und Zukunft eines Rekru- tierungspfades
}

\author{
Dissertation \\ zur Erlangung des sozialwissenschaftlichen Doktorgrades \\ der Sozialwissenschaftlichen Fakultät der Georg-August-Universität Göttingen \\ vorgelegt von \\ Kai-F. Donau \\ aus Aurich
}

Göttingen 2005 
Erbabgeordnete - Entstehung und Zukunft eines Rekrutierungspfades

1. Gutachter:

Prof. Dr. Peter Lösche

2. Gutachter:

Prof. Dr. Manfred Pohl

Tag der mündlichen Prüfung: 25.07.2006 


\section{Inhaltsverzeichnis}

$1 \quad$ Einleitung und Fragestellung ..................................................................................

$1.1 \quad$ Politische Rekrutierung ..................................................................................... 12

1.1.1 Das Konzept der Politischen Klasse................................................................. 12

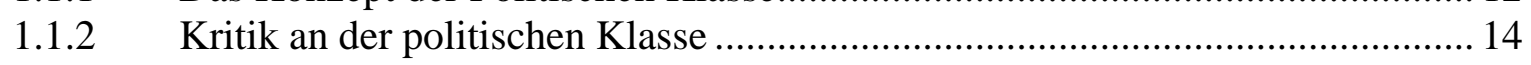

1.1.3 Politische Professionalisierung ………………………................................. 15

1.1.4 Die politische Klasse und die Opportunitätsstruktur............................................ 16

1.1.5 Rekrutierungs- und Karrierepfade..................................................................... 17

$1.2 \quad$ Anwendung auf die Fragestellung..................................................................... 21

$1.3 \quad$ Forschungsstand und Methode …………………….............................................. 23

1.3.1 Untersuchungen zu Erbabgeordneten.............................................................. 23

1.3.2 Leitfadeninterviews mit Abgeordneten ........................................................ 24

1.3.3 Auswahl der Interviewpartner ....................................................................... 26

1.3.4 Durchführung der Interviews und Auswertung................................................... 29

2 Erbabgeordnete: Entstehung und Karrierepfade............................................................. 32

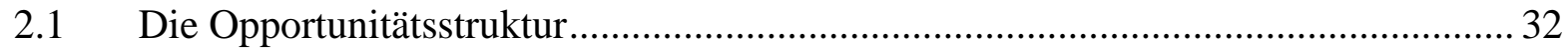

2.1.1 Das 1955er-System ……………………………………………………..... 32

2.1.1.1 Die „Eineinhalb-Parteien-Herrschaft“ der LDP ................................................. 32

2.1.1.2 Iron Triangle: Das eiserne Dreieck ................................................................. 35

2.1.2 Die Staatsstruktur ............................................................................................ 39

2.1.3 Rolle und Struktur des Parlaments.................................................................. 40

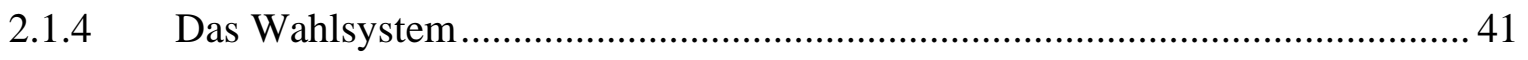

2.1.4.1 Chûsenkyokusei: Das Wahlsystem mittelgroßer Wahlkreise ............................ 41

2.1.4.2 Kôenkai: persönliche Unterstützerorganisationen der Kandidaten ................... 44

2.1.5 Parteien und Interessenverbände ..................................................................... 47

2.1.5.1 Parteien in Japan....................................................................................... 47

2.1.5.2 Zoku giin: Vermittler organisierter Interessen .................................................. 49

2.1.5.3 Habatsu: Die innerparteilichen Machtgruppen .................................................. 50

2.1.6 Die Politikfinanzierung .................................................................................. 53

2.2 Zusammenfassung: Die Opportunitätsstruktur des 1955er-Systems ..............................55

2.3 Der Rekrutierungspfad des Erbabgeordneten ............................................................. 56

2.3.1 Zur sozialen Herkunft japanischer Abgeordneter ................................................. 56

2.3.1.1 Berufsgruppen ..................................................................................... 56

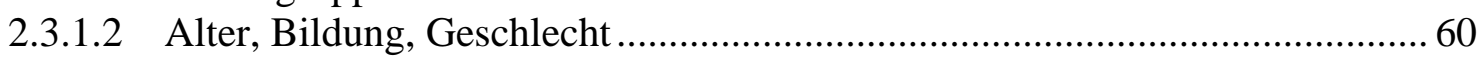

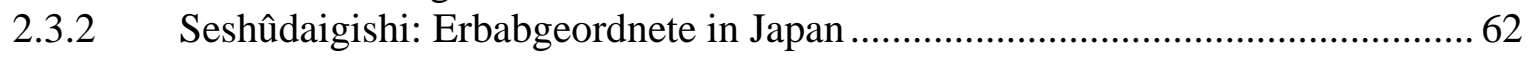

2.3.2.1 Definition und Zahl ........................................................................................62 62

2.3.2.2 Politikerfamilien und Dynastien.........................................................................6 67

2.3.2.3 Karrierewege von Erbabgeordneten ................................................................... 69

2.4 Angebot und Nachfrage: Der Rekrutierungsprozess.................................................. 73

2.4.1 Das Selbstbild der Erbabgeordneten ……………………………………….... 73

2.4.2 Die demand-Faktoren.................................................................................. 74

2.4.2.1 Auswahlmechanismen in den Parteien....................................................... 74

2.4.2.2 Die kôenkai als Selektoren ........................................................................ 78

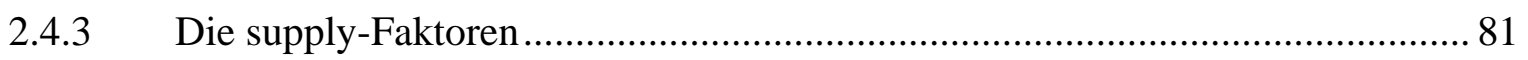

2.4.3.1 Sanban: Drei Dinge, um gewählt zu werden..................................................... 81

2.4.3.2 Ressourcen und Fähigkeiten.............................................................................. 82

2.4.3.3 Motivationen und Anreize.............................................................................. 86

2.4.3.4 Verpflichtung zum Amt: Das japanische Familiensystem................................ 87

2.5 The Outcome: Vorteile für Erbabgeordnete im 1955er-System?................................. 91 
3 Politische Reformen und Erbfolge

3.1 Seiji-kaikaku: Die Reformierung des politischen Systems ...................................... 94

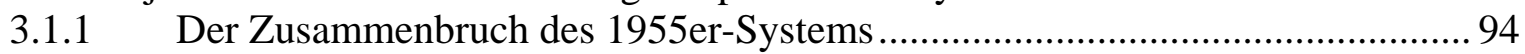

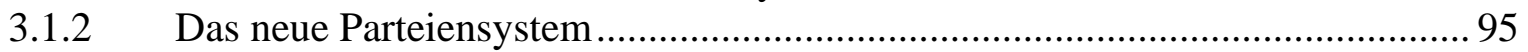

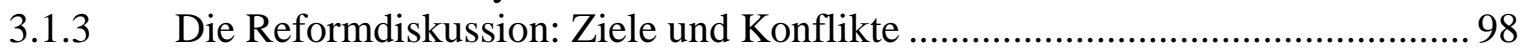

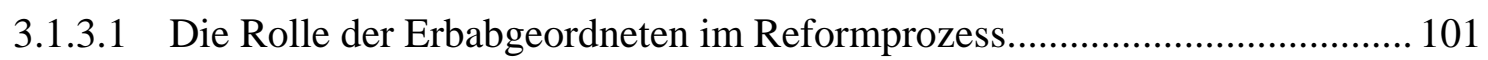

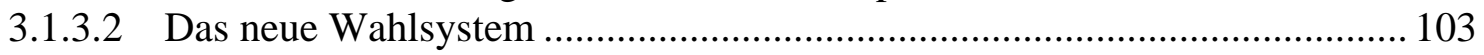

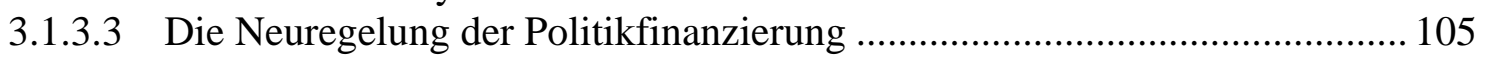

3.2 Die Auswirkungen der politischen Reformen ........................................................ 107

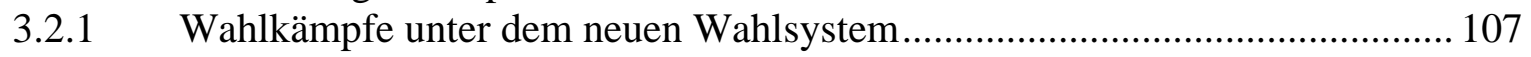

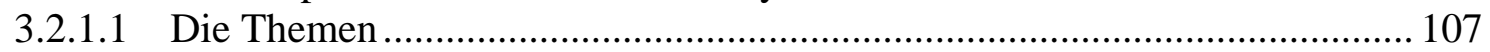

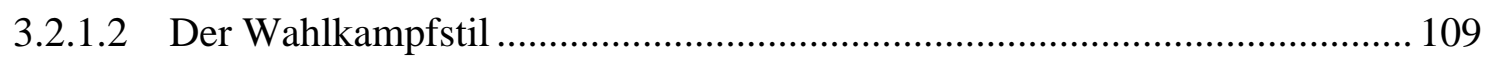

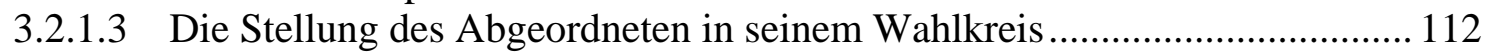

3.2.1.4 Wahlkampfkosten und Korruptionsvorfälle................................................... 113

3.2.2 Soziale Hintergründe japanischer Abgeordneter nach 1993 ............................ 116

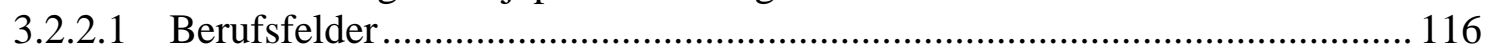

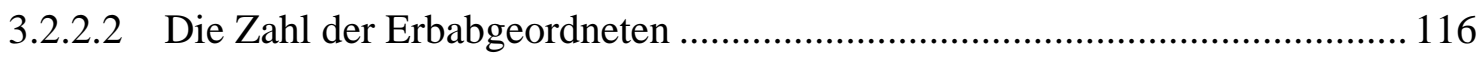

3.3 Die Veränderungen auf der Nachfrageseite ......................................................... 121

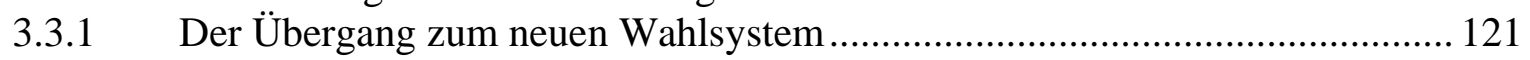

3.3.2 Vorwahlen und „öffentliche Ausschreibungen“- Neue Formen der

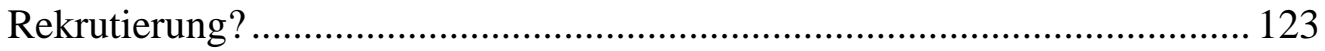

3.3.3 Machtverschiebungen bei den Selektoren..................................................... 129

3.3.3.1 Zwischen Faktion und Parteizentrale ............................................................ 129

3.3.3.2 Die Rolle der kôenkai im Rekrutierungsprozess............................................ 132

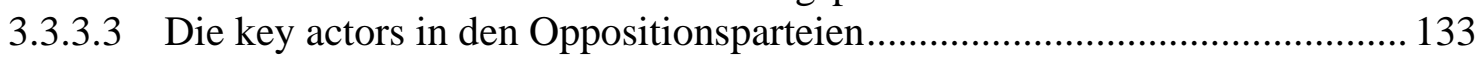

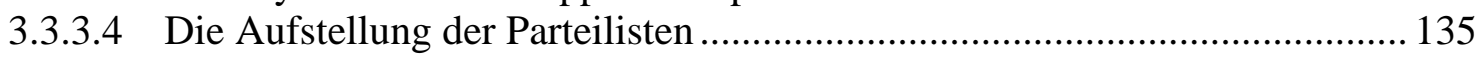

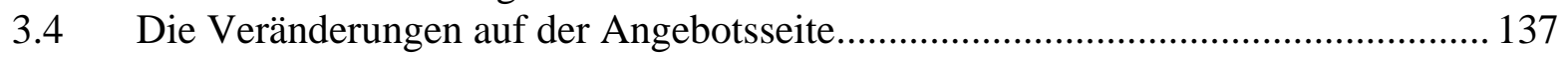

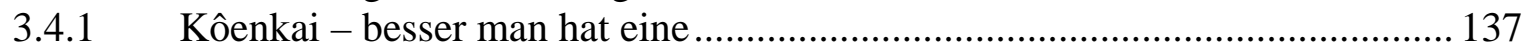

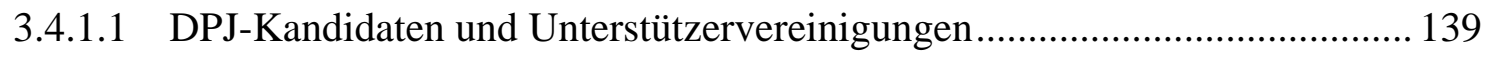

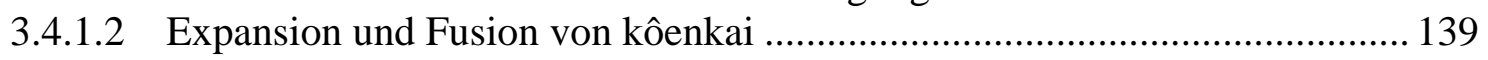

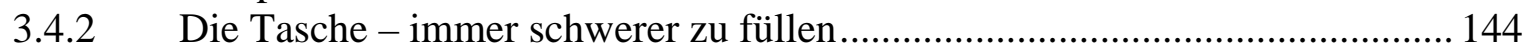

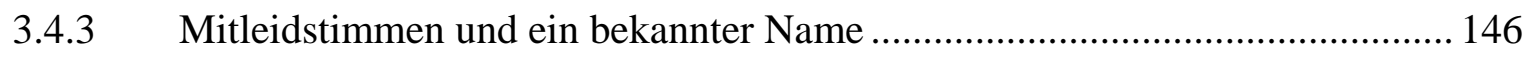

3.4.4 Der Junge vor dem Tempeltor - Das Hineinwachsen in einen Beruf ............... 148

3.5 Zusammenfassung: Veränderungen auf der Angebots- und Nachfrageseite ............ 151

4 Die Zukunft der Erbfolge in der japanischen Politik .................................................. 153

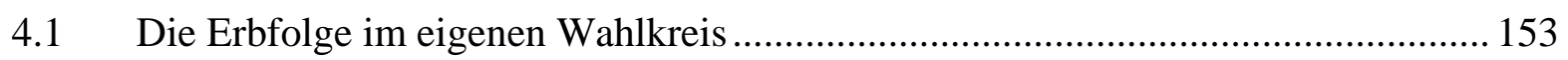

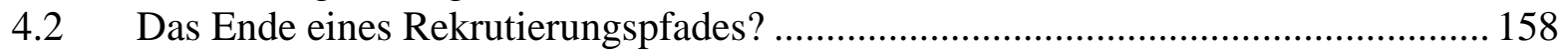

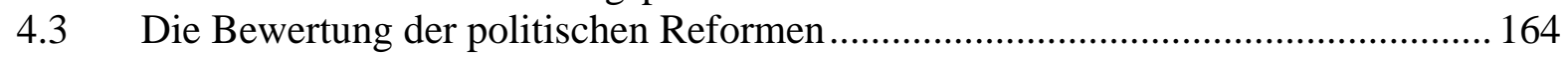

4.3.1 Unvollendet und uneffektiv - Kritik an den politischen Reformen ................... 164

4.3.2 Wer wird Politiker? - Probleme bei der Kandidatenrekrutierung..................... 166

4.4 Die Wahlen 2005: Trends in der Kandidatenaufstellung ........................................ 171

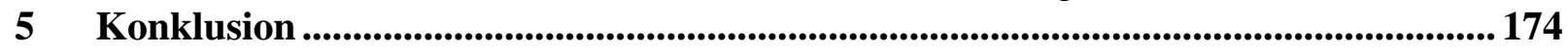

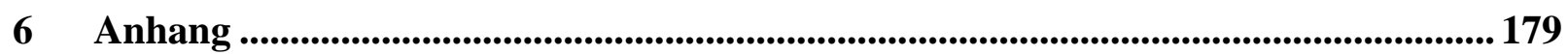

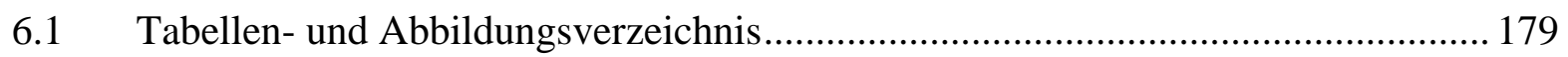

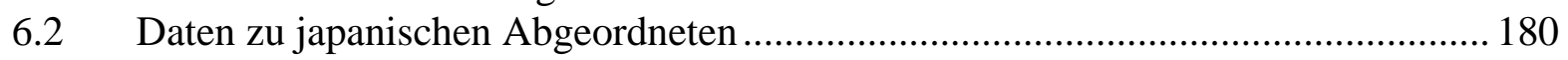

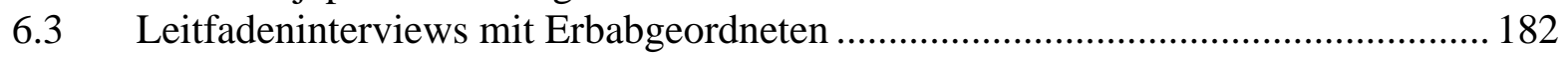

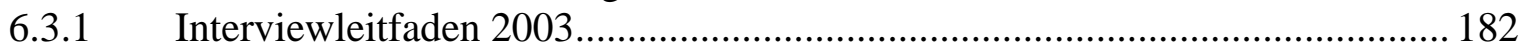

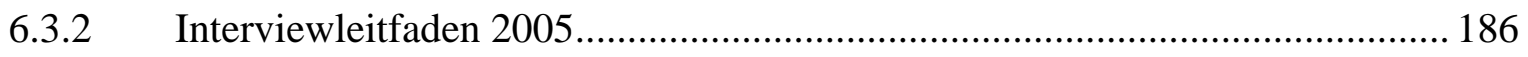

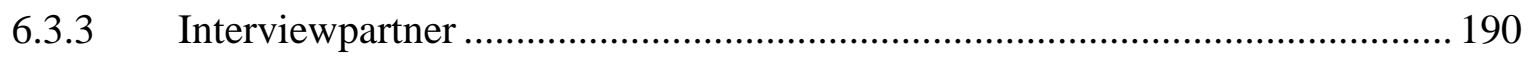


6.3.4 Beispielinterview mit Ôno Yoshinori am 21.4.2003...................................... 192

6.3.5 Beispielinterview mit Funada Hajime am 04.03.2005 ..................................... 197

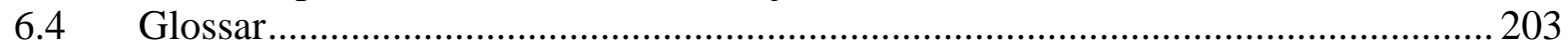

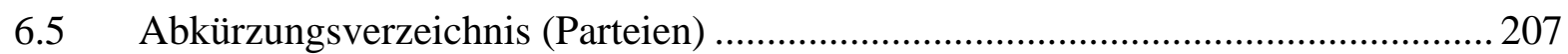

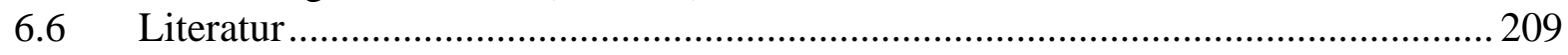

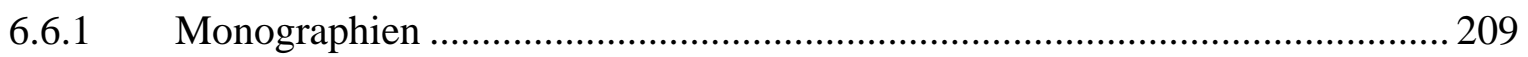

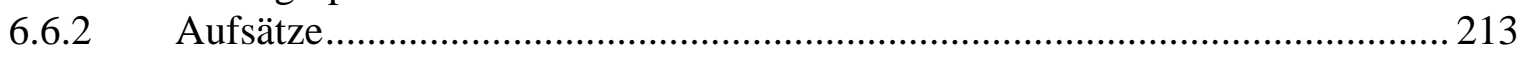

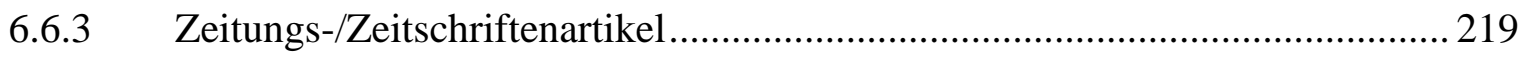

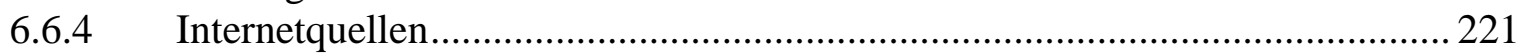

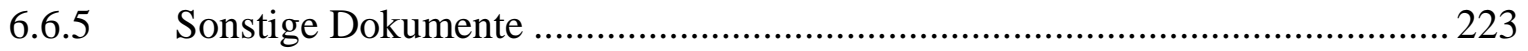

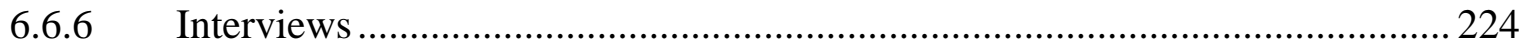




\section{Anmerkungen}

Alle japanischen Namen werden wie im Japanischen, d.h. in der Reihenfolge Familienname, Vorname wiedergegeben.

Der Wechselkurs für 100 Yen betrug zum Zeitpunkt der durchgeführten Interviews (20032005) etwa 80 Cent.

Im Anhang befindet sich ein Glossar mit einer Übersicht der wichtigsten japanischen Begriffe. 


\section{Einleitung und Fragestellung}

„The recruitment process represents one of the basic functions of every political system which has long raised normative concerns about the consequences of the recruitment process for political careers, the social diversity of legislative elites, and the democratic distribution of power within parties. " 1

Die Rekrutierung von Kandidaten für die nationalen Parlamente spielt eine zentrale Rolle in Demokratien. Hier entscheidet sich, welche Personen eine Chance bekommen, für einen Parlamentssitz zu kandidieren und eventuell ein Mandat auszuüben. Einige von ihnen werden später vielleicht einen Posten in der Regierung übernehmen oder sogar Regierungschef werden. Da ein politisches System letztlich auch von der Qualität seiner politischen Elite bzw. Klasse lebt, ist die politische Rekrutierung also von grundlegender Bedeutung: „Denn so, wie ein Konzern sich unter gutem bzw. schlechtem Management verschieden entwickelt, hängt auch die Wohlfahrt eines Gemeinwesens von der Qualität seiner Politiker ab. “²

Für die Wähler spielt die politische Rekrutierung ebenfalls eine große Rolle. Sie können bei einer Wahl nur dann ihre Zustimmung oder Ablehnung bekunden, wenn sie Alternativen haben, aus denen sie wählen können: „[...] Recruitment is a mediating phenomenon in democratic politics, connecting the mass public to governmental institutions. “3 Schließlich ist die Kandidatenauswahl auch für die Parteien selbst wichtig. Im Wahlkampf zählt nicht allein das politische Programm, sondern auch die Attraktivität der Kandidaten.

Je nach Wahlsystem, Herkunft oder Partei haben Parlamentarier jedoch unterschiedliche Fähigkeiten und Erfahrungen, die sie im Laufe ihrer Karriere gesammelt haben. Diese beeinflussen wiederum ihre späteren Entscheidungen und prägen ihre politischen Strategien. Einige Abgeordnete mussten sich auf einer „Ochsentour“ in der Partei hocharbeiten, andere hingegen sind als „politische Unternehmer“ darauf angewiesen, selbst für eine Wahlkampforganisation und die Wahlkampffinanzierung zu sorgen.

In einem politischen System bilden sich also bestimmte Rekrutierungs- und Karrieremuster heraus. Ein besonderer Karrierepfad, der sich in Japan etabliert hat, ist der des Erbabgeordne-

\footnotetext{
${ }^{1}$ Norris, Pippa: Introduction: theories of recruitment, in: Norris, Pippa: Passages to power: legislative recruitment in advanced democracies, Cambridge University Press, Cambridge 1997, S. 3.

${ }^{2}$ Patzelt, Werner J: Abgeordnete und ihr Beruf: Interviews - Umfrage - Analysen, Akad. Verlag, Berlin 1995, S. 283.

${ }^{3}$ Fowler, Linda L.: Theories of Recruitment in Comparative Perspective, Unveröff. Mskr., IPSA, Berlin 1994, S. 24.
} 
ten. Unter den 19 Mitgliedern des japanischen Kabinetts (Frühjahr 2005) befinden sich 12 Politiker, deren Väter oder Großväter bereits Abgeordnete oder sogar Regierungschefs gewesen waren. Der amtierende Ministerpräsident Koizumi Junichirô ist ebenfalls ein so genannter Erbabgeordneter. Als Erbabgeordnete werden in Japan Politiker bezeichnet, die in zweiter, dritter oder sogar vierter Generation als Abgeordnete tätig sind und häufig Wahlkreise vertreten, für die schon ihre Väter und Großväter ein Mandat ausgeübt haben. Freilich gibt es auch in anderen westlichen Demokratien Politikerdynastien, wie etwa die Bushs oder die Kennedys in den USA. In Deutschland kann man, zumindest auf regionaler Ebene, mit den Familien Goppel und Strauß ebenfalls einige Beispiele finden. Dennoch „lassen sich familiäre Beziehungen unter den Abgeordneten des japanischen Parlaments in einem Ausmaß nachweisen, wie sie wohl in modernen Demokratien westlicher Prägung selten zu finden sind.“4

Etwa 28,5\% der japanischen Unterhausabgeordneten ${ }^{5}$ kommen aus Politikerfamilien. Diese hohe Zahl von Erbabgeordneten, nicht nur auf nationaler, sondern auch auf lokaler Ebene, zeigt, dass die Erbfolge bei der politischen Rekrutierung in Japan eine große Rolle spielt: „,...] So läßt sich aufgrund der hohen Zahl der Zweitgenerationspolitiker v.a. ab den 1970er Jahren von der Herausbildung einer politischen ,Klasse' sprechen, in der sich die politische Elite Japans durch die Weitervererbung von Mandaten und die Eheschließung zwischen Politikerfamilien selbst reproduziert. “6 ${ }^{6}$, wie die Wissenschaftlerin Verena Blechinger bemerkt.

Die Praxis der Erbfolge wurde in der Vergangenheit häufig kritisiert: Die japanische Politik stünde nur Angehörigen bestimmter Bevölkerungsschichten offen, wobei bestimmte Bildungsund Berufsstrukturen, vor allem aber wirtschaftliche Aspekte und persönliche Verbindungen eine große Rolle spielen würden. Die Erbabgeordneten wurden als Teil einer modernen Oligarchie bezeichnet, als „Angehörige der Oberschicht in einer parlamentarischen Demokratie“7. Sie galten als Symbol eines intransparenten, korruptionsanfälligen politischen Systems. So wurden häufig demokratietheoretische Bedenken geäußert: „Succession by inheritance violates basic notions of democracy. The public and press tend to see the election of second-generation

\footnotetext{
${ }^{4}$ Blechinger, Verena: Politische Korruption in Japan, Institut für Asienkunde, Hamburg 1998, S. 101.

${ }^{5}$ Vgl.: Saitô, Keiko: Seikanyôran, Vol. 20, No. 47, Seisakujihô Ai Pi, Tôkyô 2002, Anhang S. 2. (Saitô, Keiko: Führer durch Politik und Bürokratie.)

${ }^{6}$ Blechinger, Verena: Politik und Familienbande - „Erbabgeordnete“ im japanischen Parlament, in:

Nachrichten der Gesellschaft für Natur- und Völkerkunde Ostasiens, Nr. 159/160, 1997, S. 85.

${ }^{7}$ Vgl.: Moroi, Kaoru: Seshûkeiei-no kenkyû, Bungeishunjû, Nr. 10/1992, S. 309. (Moroi, Kaoru: Eine Untersuchung zu „Erbhöfen“.)
} 
candidates as virtually guaranteed, offering the electorate no significant choices and threatening the legitimacy of Japanese democracy." 8

$\mathrm{Zu}$ der Frage, wie es zu dieser für westliche Demokratien ungewöhnlich hohen Zahl von Erbabgeordneten gekommen ist, gibt es eine ganze Reihe von Untersuchungen. ${ }^{9}$ Diese Studien beschäftigen sich jedoch in der Regel mit der Situation unter dem so genannten „1955erSystem“, der Zeit zwischen 1955 und 1993. Sie weisen zumeist auf den Zusammenhang zwischen der Erbfolge und den politischen Rahmenbedingungen, vor allem dem Wahlsystem, hin. Unter dem für das Unterhaus geltenden „System mittelgroßer Wahlkreise“ wurden in einem Wahlkreis bis zu fünf Abgeordnete gewählt, wobei der Wähler nur eine, nicht übertragbare, Stimme hatte (SNTV-MMD, Single-nontransferable vote - multi-member districts). Das bedeutete, dass auch Kandidaten aus der selben Partei gegeneinander antraten, was kostenintensive, personalisierte Wahlkämpfe zur Folge hatte.

Erbabgeordnete waren ein Phänomen, das man überwiegend in der von 1955 bis 1993 regierenden Liberaldemokratischen Partei (LDP) beobachten konnte. An die Person des Kandidaten gebundene Unterstützerorganisationen (kôenkai) und mangelnde lokale Parteistrukturen begünstigten eine „Erbfolge“ ebenso wie die starken informellen Netzwerke in der LDP, die so genannten habatsu, die mitunter einen großen Einfluss auf die Aufstellung der offiziellen Kandidaten nahmen. Kandidaten, die eine Erbfolge antraten, konnten außerdem zumeist größere Ressourcen vorweisen als ihre Konkurrenten. Sie verfügten in der Regel mit der Unterstützerorganisation ihrer Vorgänger über eine feste Basisorganisation, über ausreichende finanzielle Mittel und Verbindungen zu entsprechenden Sponsoren sowie über einen bekannten Namen, der in den stark personalisierten Wahlkämpfen von großer Bedeutung war.

Mitte der 1990er Jahre befand sich Japan in einem Umbruch. Die langjährige Regierungspartei LDP wurde kurzzeitig in die Opposition verbannt und im Parteiensystem kam es zu zahlreichen Parteiabspaltungen und -neugründungen. Eine Vielzahl von politischen Reformen wurde beschlossen und durchgeführt, die zu einem großen Teil das Wahlsystem und die Parteienfinanzierung betrafen. Die Mehrerwahlkreise wurden abgeschafft, stattdessen wird unter dem neuen Wahlsystem ein Teil der Abgeordneten in Einerwahlkreisen (Mehrheitswahl), ein anderer Teil der Abgeordneten über Parteilisten (Verhältniswahl) bestimmt. Auch eine staatliche Parteienfinanzierung wurde eingeführt.

Die Hoffnungen, die in diese Reformen gesetzt wurden, waren groß. Das neue Wahlsystem sollte die kostspieligen Wahlkämpfe eindämmen, die thematische und programmatische Ausei-

\footnotetext{
${ }^{8}$ Ishibashi, Michihiro; Reed, Steven R.: Second-generation diet members and democracy in Japan, in: Asian Survey, Vol. 32/1992, No. 4, S. 366.

${ }^{9}$ Vgl. die im Anhang aufgeführten Untersuchungen von Ichikawa Taiichi, Verena Blechinger und Ishibashi/Reed.
} 
nandersetzung zwischen den Parteien fördern und der starken Personalisierung in den Wahlkämpfen entgegenwirken. Durch die Einführung von Einerwahlkreisen entfiel die Notwendigkeit, mehrere Kandidaten pro Wahlkreis aufzustellen. Dadurch, so glaubten viele, würden die offiziellen Parteiorganisationen gegenüber den persönlichen Unterstützerorganisationen der Kandidaten gestärkt.

Damit betreffen die Reformen auch den Rekrutierungspfad des Erbabgeordneten. Denn nicht zuletzt gab es die Hoffnung, durch die Änderung des Wahlsystems und der Politikfinanzierung mehr Transparenz bei der politischen Rekrutierung zu schaffen und damit die Chancen für Kandidaten zu erhöhen, die keine Erbfolge antreten können. Anstelle der persönlichen Unterstützerorganisationen oder innerparteilicher Machtgruppen, die eine Erbfolge begünstigen, könnten nun die offiziellen Parteiorganisationen bzw. die Parteimitglieder einen stärkeren Einfluss auf die Kandidatenauswahl nehmen. Allerdings gab es auch erhebliche Zweifel am Erfolg der politischen Reformen im Zusammenhang mit der Erbfolge in der japanischen Politik. Einigen Wissenschaftlern erschien fraglich, ob sich durch das neue Wahlsystem eine substantielle Änderung ergeben würde. ${ }^{10}$

Es stellt sich also die Frage, inwieweit sich die Reformen des politischen Systems in Japan auf den Karrierepfad des Erbabgeordneten ausgewirkt haben. Haben die Veränderungen der institutionellen Rahmenbedingungen die Erfolgschancen von Erbpolitikern eingeschränkt? Haben sich die Chancen von Kandidaten ohne entsprechenden familiären Hintergrund verbessert? Haben die offiziellen Parteiorganisationen bei der Kandidatenrekrutierung an Einfluss gewonnen? Gibt es noch Möglichkeiten, den Karrierepfad des Erbabgeordneten einzuschlagen? Über welche Motivation verfügen Kandidaten aus Politikerfamilien, unter den aktuellen Bedingungen eine Erbfolge anzutreten?

Schließlich soll eine Prognose gewagt werden, ob der Rekrutierungspfad des Erbabgeordneten überhaupt noch zukunftsfähig ist und weiterhin erhalten bleibt.

Zu den Folgen der politischen Reformen gibt es zwar zahlreiche Untersuchungen, die sich mit dem Wahlsystem, den Veränderungen im Parteiensystem oder der Politikfinanzierung beschäftigen. Die Auswirkungen auf die Karrierewege von Abgeordneten und speziell auf den Rekrutierungspfad des Erbabgeordneten werden jedoch zumeist nur am Rande erwähnt. Ziel der vorliegenden Arbeit ist es, diese Forschungslücke zu schließen.

Aufgrund der schmalen Literaturbasis sollen qualitative Interviews, die der Verfasser mit japanischen Unterhausabgeordneten geführt hat, einen wesentlichen Teil der Materialbasis bil-

\footnotetext{
${ }^{10}$ Vgl.: Blechinger, Verena: Politik und Familienbande, a.a.O., S. 85.
} 
den. Im Frühjahr 2003 und Frühjahr 2005 wurden Erbabgeordnete zu den Veränderungen der politischen Rekrutierung und der Zukunftsfähigkeit der Erbfolge in der japanischen Demokratie befragt.

Die Analyse dieses Karrierepfades erfolgt mithilfe eines Konzepts, das Anfang der 1990er Jahre von den Politikwissenschaftlern Pippa Norris, Jonis Lovenduski ${ }^{11}$ und Linda Fowler ${ }^{12}$ entwickelt wurde. Der Rekrutierungsprozess wird in diesem Modell als „politischer Marktplatz“ begriffen, der von Angebot und Nachfrage bestimmt wird. Eingebettet in die institutionellen Rahmenbedingungen wird die politische Rekrutierung zum einen von Nachfragefaktoren, d.h. den Selektoren in Parteien, Interessengruppen oder Wahlkampforganisationen, zum anderen von Angebotsfaktoren, d.h. den individuellen Ressourcen und Motivationen der Kandidaten beeinflusst.

Da in den meisten Demokratien das nationale Parlament als das eigentliche Karriereziel für Berufspolitiker gilt, soll der Fokus der Analyse auf den Unterhausabgeordneten der beiden größten japanischen Parteien, der LDP und der Demokratischen Partei Japans (DPJ) liegen. Das japanische Parlament besteht aus zwei Kammern, dem Oberhaus (sangiin) und dem Unterhaus (shûgiin). Das Oberhaus verfügt jedoch im Vergleich zum Unterhaus über weitaus geringere Einflussmöglichkeiten. In beiden Kammern sind zwar Erbabgeordneten in großer Zahl zu finden, ihr relativer Anteil ist jedoch im Unterhaus (2002: 28,5\%) wesentlich höher als im Oberhaus (2002: 15,5\%). Hinzu kommt, dass es bei den Wahlsystemen der beiden Kammern einige Unterschiede gibt. Doch gerade das Wahlsystem des Unterhauses, das bis 1996 in Kraft war, wurde mitverantwortlich gemacht für den ausgeprägten Klientelismus in der japanischen Politik und nicht zuletzt für die hohe Zahl von Erbabgeordneten.

\footnotetext{
${ }^{11}$ Norris, Pippa; Lovenduski, Joni: Political Recruitment. Gender, race and class in the British parliament, Cambridge University Press, Cambridge 1995.

${ }^{12}$ Fowler, Linda: A.a.O.
} 


\subsection{Politische Rekrutierung}

Bevor das von Norris und Lovenduski entwickelte Modell zur Analyse politischer Rekrutierung vorgestellt wird, soll zunächst ein für das Verständnis dieses Konzepts zentraler Begriff erläutert werden. Es handelt sich um das Konzept der „Politischen Klasse“, zu der es viel Literatur und umfangreiche Studien gibt. Die folgende Darstellung basiert vor allem auf den Untersuchungen zur politischen Klasse, die im Rahmen des Zentrums für Europa- und NordamerikaStudien (ZENS) an der Universität Göttingen entstanden sind.

\subsubsection{Das Konzept der Politischen Klasse}

Wie in den meisten anderen westlichen Demokratien hat auch die japanische Politik eine Professionalisierung hinter sich, durch die eine „politische Klasse“ entstanden ist. Der Begriff der politischen Klasse, der auf den Italiener Gaetano Mosca zurückgeht, wird in Deutschland noch nicht allzu lang gebraucht. Dabei hatte die politische Klasse zunächst noch eine überwiegend negative Konnotation und wurde als Begriff eher diffamierend von den Medien oder enttäuschten Parteimitgliedern benutzt. ${ }^{13}$

Der Begriff der politischen Klasse lehnt sich stark an Max Webers Definition moderner Berufspolitiker an, die nicht mehr allein „für die Politik“, sondern auch „von der Politik“ leben: „,Von’ der Politik als Beruf lebt, wer danach strebt, daraus eine dauernde Einnahmequelle zu machen. “14 Dem Berufspolitiker geht es nicht mehr allein um Macht oder den Dienst am Gemeinwohl, er hat die Politik zu seinem Beruf gemacht. Allerdings verstand Weber die Entwicklung der Berufspolitiker als eine Entwicklung zum Parteibürokraten, wie er seinerzeit in Deutschland entstand.

Der Politikwissenschaftler Klaus von Beyme wendet den Begriff der politischen Klasse ebenfalls vor allem auf den Parteienstaat an und bezeichnet die politische Klasse als spezifisches Merkmal der Parteiendemokratie. Er benutzt diesen Begriff vor allem, um eine Abgrenzung zur „politischen Elite“ zu erreichen. Während die politische Elite sich dadurch definiert, dass ihre Vertreter wichtige Entscheidungen treffen und damit die „parlamentarischen Spitzen der Parlamentshierarchie“, aber auch Vertreter von Interessengruppen oder hohe Verwaltungsbeamte umfasst, zählen zur politischen Klasse beispielsweise auch die Hinterbänkler im Parlament, die nicht zwangsläufig an Schlüsselentscheidungen teilhaben. Sie zeichnen sich durch die Siche-

\footnotetext{
${ }^{13}$ Vgl.: Beyme, Klaus von: Der Führungsanspruch der politischen Klasse, in: Bude, Heinz; Schleissing, Stephan: Junge Eliten: Selbständigkeit als Beruf, Kohlhammer, Stuttgart 1997, S. 39.

${ }^{14}$ Weber, Max: Politik als Beruf [1919], Reclam, Stuttgart 1992, S. 16.
} 
rung ihrer Einkommen und ihrer Karrieren mit Hilfe ihres Mandats aus: „Die politische Klasse handelt selbstreferentiell und sichert ihre Position. “15

Grundsätzlich stellt von Beyme der politischen Steuerung durch die politische Elite die Selbsterhaltung der politischen Klasse gegenüber. Dabei gibt es natürlich Überschneidungen. Der Fraktionsvorsitzende einer bestimmten Partei lässt sich ebenso zur politischen Elite zählen wie auch zur politischen Klasse, da er mit der Politik seinen Lebensunterhalt verdient. ${ }^{16}$

Ein Kritikpunkt an von Beymes Definition der politischen Klasse ist, dass dieser die politische Klasse zu eng mit dem Parteienstaat verknüpft hat und es danach beispielsweise in den USA keine politische Klasse geben kann. Borchert rät daher, den Fokus einer Analyse auf das nationale Parlament zu legen, „aufgrund seiner großen Bedeutung für politische Karrieren in allen Ländern und weil es jenes Nadelöhr bildet, durch das nahezu jede institutionelle Reform, welche die politischen Karrierebedingungen verändern könnte, passieren muss. “17 Aus diesem Grund werden in der vorliegenden Arbeit die Abgeordneten auf der nationalen Ebene, genauer gesagt die Abgeordneten des japanischen Unterhauses, betrachtet.

Dennoch muss beachtet werden, dass zur politischen Klasse ein wesentlich größerer Personenkreis gezählt werden kann. Aufgrund der bereits angesprochenen Begriffsproblematik gibt es höchst unterschiedliche Zahlen zur Größe der politischen Klasse in einem bestimmten Land. Borchert und Golsch zählen zur politischen Klasse neben den Parlamentariern auch Regierungsmitglieder, hauptamtliche Parteifunktionäre, Mitarbeiter von Abgeordneten und Fraktionen oder sogar einen Teil der politischen Beamten und politisch ernannten Richter. ${ }^{18}$ Hier sehen Derichs und Kerbo, die die historische Professionalisierung in Japan untersucht haben, ein Problem: „Wenn wir sie alle dazuzählen, und außerdem noch alle Staatsbediensteten, die irgendwie an der politischen Entscheidungsfindung und -umsetzung beteiligt sind, haben wir am Ende 800.000 Mitglieder der politischen Klasse allein aus den Ministerien [...]. Offensichtlich wäre eine Definition der politischen Klasse Japans, die all diese Personen umfaßt, unrealistisch; doch wo sollte man die Grenze ziehen und wie?“19

Es gibt bei der politischen Klasse also einige Abgrenzungsprobleme, die auch Borchert und Golsch sehen. Auf diese Problematik kann im Rahmen der vorliegenden Arbeit nicht näher eingegangen werden. Es lässt sich jedoch ein gemeinsames Merkmal für die moderne politi-

\footnotetext{
${ }^{15}$ Beyme, Klaus von: Der Führungsanspruch der politischen Klasse, a.a.O., S. 43.

${ }^{16}$ Vgl.: Ebd., S. 42.

${ }^{17}$ Borchert, Jens: Politik als Beruf: Die politische Klasse in westlichen Demokratien, in: Borchert, Jens (Hg.): Politik als Beruf. Die politische Klasse in westlichen Demokratien, Leske+Budrich, Opladen 1999, S. 11.

${ }^{18}$ Vgl.: Borchert, Jens; Golsch, Lutz: Die politische Klasse in westlichen Demokratien: Rekrutierung, Karriereinteressen und institutioneller Wandel, in: Politische Vierteljahresschrift, Vol. 35/1995 S. 6111f.

${ }^{19}$ Derichs, Claudia; Kerbo, Harold: Japan. Politische Karriere zwischen Ministerialbürokratie und Erbhöfen, in: Borchert, Jens (Hg.): Politik als Beruf. Die politische Klasse in westlichen Demokratien. Reihe Europa- und Nordamerika-Studien Band 5, Leske + Budrich, Opladen 1999, S. 291.
} 
sche Klasse in repräsentativen Demokratien herausarbeiten, „das kollektive Interesse an einem stetigen und verlässlichen Einkommen aus der politischen Tätigkeit, an realistischen Chancen der Karrieresicherung sowie an Aufstiegsmöglichkeiten. “20 Das heißt, die politische Klasse zeichnet sich sowohl durch ihr kollektives Bewusstsein und ihr kollektives Handeln als auch durch ihr materielles Eigeninteresse aus. Unterschiede in Ideologie, Parteizugehörigkeit und sozialer Herkunft treten dabei in den Hintergrund. Politiker werden Akteure mit eigenen Partikularinteressen, sozusagen ihre eigene Interessengruppe. ${ }^{21}$

\subsubsection{Kritik an der politischen Klasse}

Gerade dieser Aspekt der politischen Klasse wird häufig kritisiert. In Hinblick auf andere Berufsfelder kann man von der politischen Klasse nämlich sagen: „Niemand sonst hat so viel Einfluß auf die Ausgestaltung des eigenen Umfeldes. “22 Da die politische Klasse als kollektiver Akteur mit kollektiven Interessen, beispielsweise einem „Selbsterhaltungsinteresse“, auftritt, wird sie versuchen, die politischen Rahmenbedingungen oder Opportunitätsstrukturen so zu gestalten, dass ihre Karrieren gesichert sind. ${ }^{23}$

Daher hat die politische Klasse in vielen Teilen der Bevölkerung ein negatives Image: Berufspolitiker gelten als abgehoben und haben sich, so der Vorwurf, von den Wählern zu weit entfernt. Vor allem die Tatsache, dass die politische Klasse ihre Rahmenbedingungen so stark beeinflussen kann, ist Anlass zur Kritik. Hans Herbert von Arnim etwa spricht davon, dass die Eigeninteressen der politischen Klasse „alle Innovationsbestrebungen zu ersticken drohen. “24

Auch wenn diese Kritik nicht selten überzogen wirkt, macht sie doch deutlich, dass bei politischen Reformen eine Wechselwirkung zwischen den institutionellen Veränderungen und dem Eigeninteresse der Politiker besteht. Zum einen finden Reformen statt, wenn es ein Eigeninteresse wie etwa Einkommen oder Karrieresicherung der politischen Klasse gibt, die institutionellen Rahmenbedingungen (z.B. die Höhe der Diäten) zu verändern. Zum anderen können Reformen unter großem äußeren Druck stattfinden, besonders in schweren Krisen des politischen Systems. „Historisch sind jedoch die Reformen der zweiten Art weitaus weniger erfolgreich gewesen als jene, die entweder durch das Eigeninteresse der politischen Klasse motiviert waren oder aber sich doch wenigstens in ein solches Interesse übersetzen ließen. “25

\footnotetext{
${ }^{20}$ Borchert, Jens: Politik als Beruf, a.a.O., S. 9.

${ }^{21}$ Vgl.: Borchert, Jens; Golsch, Lutz: Die politische Klasse in westlichen Demokratien, a.a.O., S. 615.

${ }^{22}$ Ebd., S. 615.

${ }^{23}$ Vgl.: Beyme, Klaus von: Der Begriff der politischen Klasse - eine neue Dimension der Elitenforschung? In: Politische Vierteljahresschrift, Jg. 33, Heft 1/1993, S. 29.

${ }^{24}$ Arnim, Hans Herbert von: Fetter Bauch regiert nicht gern. Die politische Klasse - selbstbezogen und abgehoben, Knaur, München 1999, S. 19.

${ }^{25}$ Borchert, Jens: Politik als Beruf, a.a.O., S. 32.
} 
Ziel der Arbeit ist es zwar nicht, die Reformdiskussion der 1990er Jahre in Japan und die Rolle der Erbabgeordneten in diesem Reformprozess zu analysieren. Dennoch darf bei der der Betrachtung der Veränderungen im Wahlsystem bzw. der Politikfinanzierung dieser Gesichtspunkt nicht vergessen werden.

\subsubsection{Politische Professionalisierung}

Den Typus des Berufspolitikers sieht der Göttinger Politologe Jens Borchert als Folge eines Professionalisierungsprozesses, der je nach Land unterschiedlich verläuft. In einer Studie hat er Professionalisierungsmuster in den USA und Deutschland miteinander verglichen. ${ }^{26}$ Für eine Professionalisierung gibt es demnach strukturelle Vorraussetzungen. Dazu zählt zum einen ein verlässliches Einkommen, von dem der Berufspolitiker leben kann und das zumindest ansatzweise über eine Attraktivität im Vergleich zu anderen Berufen verfügt. Eine andere Vorraussetzung ist die „realistische Chance, über längere Zeit eine Tätigkeit in der Politik behalten zu können. “27 Diese Chancen können sowohl in relativ sicheren Mandaten (z.B. Listenplätzen) bestehen als auch in Auffangpositionen, in die der Berufspolitiker wechseln kann, wenn er sein Mandat verliert.

Als Drittes werden die Aufstiegsmöglichkeiten genannt, die vorhanden sein müssen. Dabei wird davon ausgegangen, dass ein beruflicher Ehrgeiz vorhanden ist, auf der politischen Karriereleiter aufzusteigen, sei es innerhalb oder zwischen verschiedenen Institutionen: ${ }^{28}$ „Zu einer dauerhaften politischen Professionalisierung und der Herausbildung einer politischen Klassedie notwendigen Vorraussetzungen sind hier die gleichen - kommt es historisch dann, wenn eine wachsende Zahl von Menschen sich unter den gegebenen Rahmenbedingungen entscheidet, Politik zu ihrem Beruf zu machen, für längere Zeit dort zu verbleiben und dabei die Rahmenbedingungen ihren Bedürfnissen anzupassen. “29

Von Beyme spricht von drei Indikatoren, die eine politische Professionalisierung anzeigen: ${ }^{30}$

o Die Zahl der Jahre im Beruf vor Erreichung des ersten wichtigen politischen Amtes.

o Die berufliche Nebentätigkeiten neben den politischen Ämtern.

o Die Bereitschaft zur Rückkehr in den Ausgangsberuf.

Der letzte Punkt ist nicht ohne Bedeutung. Von Beyme merkt an, „dass die Arbeit als Anwalt, wie sie sich Barzel, Ehmke oder Lambsdorff noch als Option offenhielten, selbst für Juristen heute nicht mehr sehr üblich ist. Üblicher ist es, Auffangpositionen für die Abgänger zu

\footnotetext{
${ }^{26}$ Vgl.: Borchert, Jens: Pathways to Professionalism: Institutional Context and the Emergence of a Political Class in the U.S. and Germany, Unveröff. Mskr., APSA, Washington, D.C. 1997, S. 3.

${ }^{27}$ Borchert, Jens: Politik als Beruf, a.a.O., S. 15.

${ }^{28}$ Vgl.: Ebd., S. 15.

${ }^{29}$ Ebd., S. 15.

${ }^{30}$ Vgl.: Beyme, Klaus von: Die politische Klasse im Parteienstaat, 2. Aufl., Suhrkamp, Franfurt/M. 1995, S. 120
} 
schaffen. “31 Wenn es, wie am Fall Japan noch gezeigt wird, wenig Auffangpositionen beim Ausscheiden aus dem Amt gibt, geht es weniger um die Bereitschaft als um die Möglichkeit zur Rückkehr in den Ausgangsberuf, und man spricht dann von einer mangelnden sozialen Mobilität.

\subsubsection{Die politische Klasse und die Opportunitätsstruktur}

Die politische Professionalisierung kann sowohl auf einer Makro- wie auch auf einer Mikroebene betrachtet werden: Die Makroebene beschreibt die historische Professionalisierung, die Mikroebene die Professionalisierung(smuster) der einzelnen Abgeordneten. ${ }^{32}$ Zwei Forschungsperspektiven stehen sich dabei gegenüber: der historisch-institutionelle Ansatz und der individuell-longitudinale Ansatz.

Der historisch-institutionelle Ansatz stellt vor allem die Legislativen und deren strukturelle Veränderungen in den Mittelpunkt. Ziel ist die Analyse von Professionalisierungsprozessen, beispielsweise die Entwicklung vom Typus des Amateurpolitikers oder des Honoratioren zum Berufspolitiker. Der zweite Ansatz dagegen richtet seinen Fokus auf den einzelnen Politiker. Individuelle Karriereverläufe werden zu Mustern (pattern) verdichtet, ausgehend von der Annahme, „dass es in einem politischen System nicht beliebig viele verschiedene Aufstiegswege gibt, sondern dass diese durch den gesellschaftlichen und institutionellen Rahmen beschränkt sind. "33

Borchert und Golsch üben Kritik vor allem an einer fehlenden Verknüpfung dieser beiden Ansätze. Die beiden Ansätze müssten zusammengeführt werden, um die Schwächen der jeweils anderen Forschung auszugleichen. Die Analyse einzelner Karriereverläufe und der persönlichen Ambitionen der Berufspolitiker sei mit den gesellschaftlichen und institutionellen Rahmenbedingungen verknüpft. ${ }^{34}$

Diese Rahmenbedingungen, auch Opportunitätsstruktur genannt, umfassen bei Borchert sechs Bereiche: die Staatsstruktur, die Rolle des Parlaments, die Parlamentsstruktur, das Wahlsystem, die Rolle von Parteien und Verbänden und die Politikfinanzierung. Diese Opportunitätsstruktur prägt auch den jeweiligen Berufspolitikertypus. Der politische Unternehmer („Entrepreneur“) und der Parteipolitiker („Hinterbänkler“) bilden dabei Idealtypen. ${ }^{35}$

Die Opportunitätsstrukturen beeinflussen die politische Klasse sowohl individuell als auch kollektiv, d.h. sowohl den Karrierepfad des einzelnen Politikers als auch die Karrieremuster in

\footnotetext{
${ }^{31}$ Beyme, Klaus von: Die politische Klasse im Parteienstaat, a.a.O., S. $120 \mathrm{f}$.

${ }^{32}$ Vgl.: Borchert, Jens: Politik als Beruf, a.a.O., S. 16-17.

${ }^{33}$ Borchert, Jens; Golsch, Lutz: Die politische Klasse in westlichen Demokratien, a.a.O., S. 616-617.

${ }^{34}$ Vgl.: Ebd., S. 617.

${ }^{35}$ Vgl.: Borchert, Jens: Politik als Beruf, a.a.O., S. 20-23.
} 
einem politischen System. Die politische Klasse wiederum kann ihrerseits bis zu einem gewissen Grad die Rahmenbedingungen des politischen Systems beeinflussen. Gerade auf nationaler Ebene ist es möglich, auf die eigenen Opportunitätsstrukturen wie etwa die Politikfinanzierung Einfluss zu nehmen. ${ }^{36}$

\subsubsection{Rekrutierungs- und Karrierepfade}

Bei der Betrachtung von politischen Karrieren steht daher folgende Frage im Zentrum des Interesses, „[...] Who, how and why people become politicians, and the consequences for parties, legislatures and representatives. “37 Grundlegende Fragestellung bei der Analyse politischer Rekrutierung ist also, wer Kandidat bzw. Abgeordneter wird, wie und warum dies passiert. Dabei gibt es das bereits erwähnte Problem, dass so genannte macro-level theories der Analyse des institutionellen oder gesellschaftlichen Kontextes und micro-level theories der Analyse von Motivationen oder Strategien einzelner Abgeordneter häufig nebeneinander stehen. Eine Verbindung zwischen diesen Ansätzen wurde selten hergestellt, obwohl keine Forschungsrichtung allein befriedigende Antworten auf die Fragen des Rekrutierungsprozesses geben konnte. ${ }^{38}$

Unter maßgeblichem Einfluss der Politikwissenschaftler Pippa Norris, Joni Lovenduski und Linda Fowler ist ein Konzept zur Analyse politischer Rekrutierung entstanden, das diese Forschungstraditionen miteinander verbindet und die Interaktion zwischen den Kandidaten und den institutionellen Rahmenbedingungen betont. Die Rekrutierung von Kandidaten, der „critical step as individuals move from lower levels into parliamentary careers”39, wird dabei als ein von Angebot und Nachfrage bestimmter „politischer Markplatz“ begriffen: Die demand side, d.h. der Rekrutierungsprozess innerhalb der Parteien, wird mit der supply side zusammengeführt, die die individuellen Motivationsfaktoren und Ressourcen der Kandidaten analysiert. Die Analyse politischer Rekrutierung wird also im Wesentlichen von zwei Fragen geleitet: Wer wählt (die Kandidaten) aus und wie? Wer wird gewählt und warum? ${ }^{40}$

Analytisch unterscheidet Norris vier Ebenen: ${ }^{41}$ Die erste Ebene umfasst die institutionellen Rahmenbedingungen bzw. Opportunitätsstruktur, die auch als broad context bezeichnet werden. Dazu gehören beispielsweise das Wahlsystem oder rechtliche Bestimmungen zur Kandidatur, „, which structure candidate opportunities in the political market-place “42.

\footnotetext{
${ }^{36}$ Vgl.: Borchert, Jens: Pathways to Professionalism, a.a.O., S. 18-23.

${ }^{37}$ Norris, Pippa: Through the Eye of the Eeedle: Comparative Recruitment in Western Democracies, Unveröff. Mskr., APSA, Washington 1993, S. 1.

${ }^{38}$ Vgl.: Fowler, Linda: A.a.O., S. 2.

${ }^{39}$ Pippa, Norris: Introduction: theories of recruitment, a.a.O., S. 1.

${ }^{40}$ Vgl.: Norris, Pippa; Lovenduski, Joni: Political Recruitment, a.a.O., S. 2ff.; Norris, Pippa: Through the Eye of the Needle: Comparative Political Recruitment in Western Democracies, a.a.O., S. 2.

${ }^{41}$ Vgl.: Pippa, Norris: Introduction: theories of recruitment, a.a.O., S. 1.

${ }^{42}$ Pippa, Norris: Introduction: theories of recruitment, a.a.O., S. 1.
} 
Auf einer zweiten Ebene wird der Rekrutierungsprozess an sich untersucht. Zunächst werden die formellen und informellen Regeln betrachtet, die z.B. in den Parteien zur Bestimmung von Kandidaten gelten. So kann es beispielsweise Vorschriften von staatlicher Seite zur Auswahl von Kandidaten geben. Diese allein stellen allerdings selten eine Erklärung für Rekrutierungsmuster dar. ${ }^{43}$

Diese zweite Ebene wird deshalb in zwei weitere Ebenen unterteilt. Auf der demand-side werden gewissermaßen die „Nachfragefaktoren“ untersucht. Diese werden im wesentlichen von den Selektoren oder gatekeepers bestimmt. Selektoren können sowohl Mitglieder lokaler Parteiorganisationen sein, die den Kandidaten in ihrem Wahlkreises bestimmen, wie auch Vertreter von Interessenverbänden oder die Parteiführung. Zunächst geht es darum, die Schlüsselakteure (key actors) im Rekrutierungsprozess zu bestimmen, wobei es durchaus parteiinterne Konflikte zwischen mehreren Akteuren geben kann, die gleichzeitig den Anspruch auf die Kandidatenbestimmung erheben. In einem zweiten Schritt werden die Rekrutierungsmechanismen analysiert, die in einer Partei, Wahlkampforganisation oder dergleichen existieren. ${ }^{44}$ Es macht jedoch Sinn, die Betrachtung der formellen und informellen Regeln in die Analyse der Nachfrageseite zu integrieren, da die formellen und informellen Bestimmungen zur Kandidatenauswahl z.B. eng mit den key actors zusammenhängen können.

Je nach Partei oder politischem System bestimmen unterschiedliche Selektoren und Mechanismen den Rekrutierungsprozess. Nach Pippa Norris lassen sich jedoch grundsätzlich vier Typen der Rekrutierung unterscheiden:

o Bei der informell-zentralisierten Rekrutierung findet die Auswahl von Kandidaten durch Aushandlungen zwischen den Partei-Eliten und rivalisierenden innerparteilichen Gruppen (factions) ${ }^{45}$ auf zentraler Ebene statt. Diese Gruppen, z.B. Honoratioren oder innerparteiliche Machtgruppen wie die japanischen habatsu, auf die später noch eingegangen wird, bestimmen die Auswahl und Aufstellung der offiziellen Kandidaten, die eventuell auch finanziell von diesen Gruppen unterstützt werden.

o Im Fall einer informell-lokalisierten Rekrutierung existieren kaum allgemeingültige Mechanismen für die Auswahl von Kandidaten. Die Auswahl wird von lokalen Organisationen vorgenommen. Hier kann es in einem politischen System eine ganze Reihe unterschiedlicher Auswahlmechanismen geben.

\footnotetext{
${ }^{43}$ Vgl.: Norris, Pippa: Through the Eye of the Needle, a.a.O., S. 8.

${ }^{44}$ Vgl.: Ebd., S. $16 f f$.

${ }^{45}$ Hier wird das englische Wort faction verwendet. Im Folgenden soll für den Fall Japan vor allem das japanische Wort habatsu verwendet werden, um Verwechslungen des in der deutschen Literatur hin und wieder benutzten Begriffs „Faktion“ mit dem Begriff „Fraktion“ zu vermeiden.
} 
o Dagegen sind bei der formell-lokalisierten Rekrutierung verbindliche Regeln zur Kandidatenauswahl vorhanden. Die Auswahl wird aber von lokalen Organisationen vorgenommen. Als Beispiel für diesen Typus nennt Norris Pippa die deutschen Sozialdemokraten.

o Den vierten Typus bildet die formell-zentralisierte Rekrutierung. Hier werden die Kandidaten von den Parteiführern oder von den faction leaders bestimmt. Es existieren gesetzliche Bestimmungen, die den Partei-Eliten diese Funktion zuschreiben.

Die Liberaldemokratische Partei Japans ordnet Pippa Norris in diesem Modell der formellzentralisierten Rekrutierung zu. Inwieweit diese Einordnung, die sich auf die Kandidatenrekrutierung der LDP unter dem 1955er-System Wahlsystem bezieht, zutrifft, wird später noch kurz zu erörtern sein. Zunächst soll diese Einordnung nur der Verdeutlichung des Modells dienen.

\section{Abbildung 1: Typen politischer Rekrutierung}

\begin{tabular}{|c|c|c|}
\hline & $\begin{array}{l}\text { centralised } \\
\text { decisions }\end{array}$ & $\begin{array}{l}\text { localised } \\
\text { decisions }\end{array}$ \\
\hline $\begin{array}{l}\text { formal } \\
\text { rules }\end{array}$ & $\begin{array}{l}\text { Dutch VVD } \\
\text { French PC } \\
\text { Italian PCI } \\
\text { Japan LDP }\end{array}$ & $\begin{array}{l}\text { British Conservatives } \\
\text { German SPD } \\
\text { Swedish SDP } \\
\text { Dutch PvdA }\end{array}$ \\
\hline $\begin{array}{l}\text { informal } \\
\text { rules }\end{array}$ & $\begin{array}{l}\text { Italian DC } \\
\text { Italian PSI } \\
\text { French UDP }\end{array}$ & $\begin{array}{l}\text { US Democrats } \\
\text { Canadian Liberals }\end{array}$ \\
\hline
\end{tabular}

Nach: Norris, Pippa: Through the Eye of the Needle: Comparative Political Recruitment in Western Democracies, Unveröff. Mskr., APSA, Washington 1993, S. 35.

Zur demand side zählen nicht nur die Erwartungen der Selektoren in den Parteien. Auch die Erwartungen der Wähler an die Kandidaten müssen berücksichtigt werden. Mitunter haben sie, etwa bei der informell-lokalisierten oder, unter bestimmten Umständen, bei der formelllokalisierten Rekrutierung einen direkten Einfluss auf die Auswahl der Kandidaten. ${ }^{46}$

Die individuellen Faktoren, die bei der politischen Rekrutierung eine Rolle spielen, werden auf der vierten Ebene, der supply side, analysiert. Hier wird untersucht, über welche Motivation und Ressourcen die einzelnen Kandidaten verfügen. Zu den Ressourcen zählen nicht nur die

\footnotetext{
${ }^{46}$ Vgl.: Norris, Pippa: Through the Eye of the Needle, a.a.O., S. 17-22; Fowler, Linda: A.a.O., S. 25.
} 
finanziellen Mittel, sondern auch die vorhandenen Netzwerke, die politischen Erfahrungen oder die Zeit, die ein Kandidat investieren kann. Die Bedeutung der einzelnen Ressourcen unterscheidet sich je nach politischem System oder Partei. ${ }^{47}$

Abbildung 2: Rekrutierungsmodell nach Norris

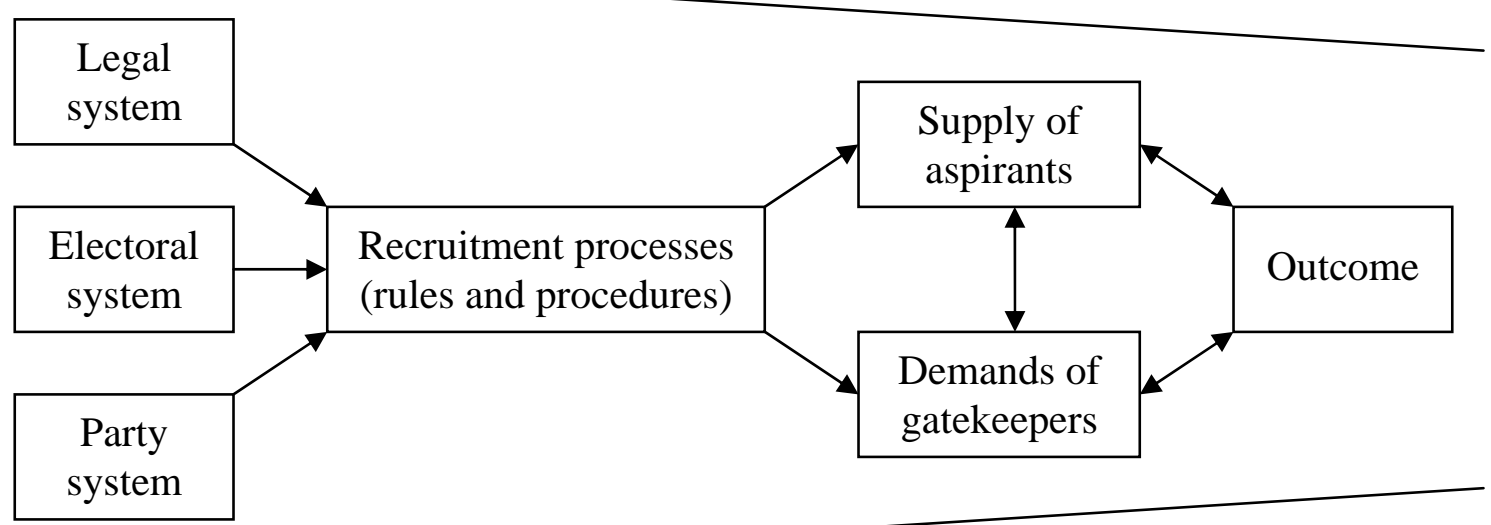

Nach: Norris, Pippa: Introduction: theories of recruitment, in: Norris, Pippa: Passages to power: legislative recruitment in advanced democracies, Cambridge University Press, Cambridge 1997, S. 4.

Zum Bereich der Motivation kann man sowohl Machtstreben und finanziellen Gewinn als auch ideologische Ziele oder Loyalitäten gegenüber bestimmten Gruppen zählen. Im Fall Japans ist, wie noch gezeigt wird, auch die Verpflichtung gegenüber der Familie oder einer Interessengruppe ein wichtiger Motivationsfaktor. Hinzu kommen die beruflichen Anreize, die eine politische Karriere bietet. Das Fortkommen auf etablierten Karrierepfaden kann für einige Gruppen von Kandidaten, beispielsweise aufgrund vorhandener Netzwerke, einfacher sein als für andere und somit eine wichtige Motivation darstellen zu kandidieren. Die vier Ebenen, der broad context, der recruitment process, die demand- und die supply side, bilden einen Trichter, einen „funnel of causality“, durch den die Personen gelangen müssen, um für ein Amt kandidieren zu können.

\footnotetext{
${ }^{47}$ Vgl.: Norris, Pippa: Through the Eye of the Needle, a.a.O., S. 23-24.
} 


\subsection{Anwendung auf die Fragestellung}

Der Vorteil des Modells von Norris und Lovenduski liegt darin, dass der Abgeordnete nicht nur unter dem Aspekt gesellschaftlicher Interessenkonstellationen oder politischer Machtverhältnisse betrachtet wird, sondern auch unter dem Aspekt des Eigeninteresses: „We see candidates for public offices as imbedded in social and institutional contexts that inhibit or encourage their political ambitions, but we also recognize the deliberate choices that bring them into the arena or keep them on the sidelines. In effect, they are both products of a particular political milieu and purposive actors. " ${ }^{48}$ Nicht nur die institutionellen Rahmenbedingungen sind bei der Rekrutierung von Bedeutung, sondern auch die persönlichen Ressourcen und Motivationen des einzelnen Kandidaten.

Dieses Modell erscheint deshalb gut geeignet, veränderte Rekrutierungsbedingungen und ihre Auswirkungen auf bestimmte Karrieremuster zu analysieren. Da es ursprünglich entwickelt wurde, um Rekrutierungsprozesse in unterschiedlichen politischen Systemen zu analysieren, lässt es sich problemlos auf den Fall Japan anwenden und auf die in der Einleitung genannte Fragestellung, wie sich die politischen Reformen in Japan auf den Rekrutierungspfad des Erbabgeordneten ausgewirkt haben.

Zunächst soll die Entstehung dieses speziellen Karrierepfades unter dem so genannten 1955er-System analysiert werden. Unter welchen Bedingungen konnte sich der Rekrutierungspfad des Erbabgeordneten etablieren? Mit Hilfe des genannten Modells soll erklärt werden, warum Erbabgeordnete unter dem 1955er-System Vorteile auf der Angebots- bzw. Nachfrageseite gegenüber anderen Kandidaten hatten und sie deshalb im japanischen Unterhaus so zahlreich vertreten waren. Hierbei ist es wichtig, den Begriff „Erbabgeordneter“ genauer zu betrachten und zu definieren (s. 2.2.2). In diesem Teil der Arbeit kann auf eine große Zahl von Studien und Publikationen zu diesem Thema zurückgegriffen werden.

Der Fokus der Analyse liegt jedoch auf der Entwicklung dieses Rekrutierungspfades nach den politischen Reformen Mitte der 1990er Jahre. Da es hierzu, wie bereits erwähnt, relativ wenig Material gibt, basiert dieser Teil der Untersuchung vor allem auf den Leitfadeninterviews, die mit Erbabgeordneten geführt wurden.

Ausgehend von den veränderten institutionellen Rahmenbedingungen wird der Rekrutierungspfad des Erbabgeordneten zuerst auf der demand-side analysiert. Welche Veränderungen hat es bei der Kandidatenrekrutierung gegeben? Wie werden in japanischen Parteien, in diesem Fall in der LDP und DPJ, die Kandidaten derzeit ausgewählt? Wer sind die Selektoren? Hat es Machtverschiebungen gegeben?

\footnotetext{
${ }^{48}$ Fowler, Linda: A.a.O., S. 5.
} 
Anschließend werden die Veränderungen des Rekrutierungsprozesses auf der supply side untersucht. Gibt es heute noch Gründe für Abgeordnete, den gleichen Beruf wie ihre Eltern auszuüben bzw. deren Wahlkreis zu übernehmen? Über welche Ressourcen und Motivationen verfügen Erbpolitiker unter den aktuellen Bedingungen, und welche Vorteile haben sie gegenüber ihren Konkurrenten? Schließlich soll eine Prognose über die Zukunftsfähigkeit dieses Rekrutierungsweges gewagt werden: Bleibt der Karrierepfad des Erbabgeordneten weiterhin erhalten?

Anhand dieses speziellen Karrieremusters kann außerdem geprüft werden, ob Veränderungen der institutionellen Rahmenbedingungen bzw. auf der demand side ausreichen, Rekrutierungsprozesse transparenter zu gestalten. Haben die veränderten Rekrutierungsmechanismen dazu geführt, dass die Politik einem größeren Personenkreis offen steht? Oder wurde nicht vielmehr die Bedeutung der supply side, also die im Verhältnis zu anderen Kandidaten immer noch sehr gute Ressourcenausstattung von Erbabgeordneten, unterschätzt, so dass die Reformen unter diesem Aspekt keine Veränderungen mit sich gebracht haben? 


\subsection{Forschungsstand und Methode}

\subsubsection{Untersuchungen zu Erbabgeordneten}

Für die Zeit von 1955 bis 1993, des so genannten 1955er-Systems, gibt es eine ganze Reihe von Studien zum Thema Erbabgeordnete. Da das Phänomen der Erbpolitiker vor allem seit den 1960er und 1970er Jahren zugenommen hat, stammen die meisten Studien aus den 1980er und frühen 1990er Jahren. Eine der wichtigsten Studien ist „seshûdaigishi-no kenkyû“ („Forschung zu Erbabgeordneten“) von Ichikawa Taiichi aus dem Jahr 1990. ${ }^{49}$ In Deutschland hat sich vor allem Verena Blechinger mit dem Thema der Erbpolitiker unter dem Gesichtspunkt der politischen Korruption beschäftigt. Von dem Japanologen Patrick Köllner stammen zahlreiche Untersuchungen zur informellen Politik wie etwa den kôenkai und den habatsu, die wie bereits erwähnt einen großen Einfluss auf die Kandidatenrekrutierung haben.

Zu den aktuellen Veränderungen des politischen Systems in Japan gibt es viele Studien, die sich mit dem Reformprozess und seinen Folgen beschäftigen. Dabei bieten die politischen Reformen in Japan Mitte der 1990er Jahre eine gute Möglichkeit, bestehende Theorien auf ihre Gültigkeit hin zu überprüfen, wie Köllner bemerkt. ${ }^{50}$ In diesem Sinne ist die Relevanz des Themas nicht auf Japan begrenzt. Allerdings spielen die Auswirkungen der politischen Reformen auf die politische Rekrutierung und den Karrierepfad der Erbabgeordneten in den bereits existierenden Studien nur eine marginale Rolle.

Abgesehen von einigen kürzeren Analysen zu den Folgen der Reformen für die Kandidatenauswahl in den einzelnen Parteien, in deren Rahmen auch die Erbfolge erwähnt wird, gibt es kaum umfangreichere Untersuchungen zu diesem Thema. Hier besteht ein Forschungsdefizit, das durch diese Arbeit teilweise aufgearbeitet werden kann.

Eine Ausnahme stellt die Untersuchung von Park Cheol Hee dar, der die Veränderungen der Wahlkampfführung in Tôkyôter Wahlkreisen untersucht hat und dabei auch auf die politische Rekrutierung eingeht. ${ }^{51}$ Zwar gibt es in dieser Untersuchung einige Anmerkungen zu Erbabgeordneten, der Fokus dieser Untersuchung liegt jedoch auf den Wahlkamfpstrategien der Abgeordneten und konzentriert sich auf die lokale und regionale Ebene.

\footnotetext{
${ }^{49}$ Ichikawa Taiichi: Seshûdaigishi-no kenkyû. Nihon keizai shinbunsha. Tôkyô 1990. (Ichikawa Taiichi: Forschung zu Erbabgeordneten.)

${ }^{50}$ Vgl.: Köllner, Patrick: Drei Jahre nach den politischen Reformen - Eine Zwischenbilanz. Teil 1: Das Wahlgesetz und seine Auswirkungen, in: Japan aktuell. Wirtschaft. Politik. Gesellschaft, Heft April/1998, S. 169.

${ }^{51}$ Vgl.: Park Cheol Hee: Daigishi-no tsukurikata. Shosenkyoku-no senkyo-senryaku, Bunshushinsho, Tôkyô 2000. (Park Cheol-Hee: Wie Abgeordnete gemacht werden. Wahlkampfstrategien in den Einerwahlkreisen.)
} 


\subsubsection{Leitfadeninterviews mit Abgeordneten}

Da die vorhandene Literatur die Auswirkungen der politischen Reformen auf die Erbabgeordneten nur am Rande behandelt und es kaum neuere Untersuchungen zu diesem Thema gibt, wurden qualitative Interviews mit japanischen Unterhausabgeordneten durchgeführt. Diese Interviews entstanden im Rahmen von zwei Forschungsaufenthalten 2003 und 2005, die jeweils durch DAAD-Kurzzeit-Stipendien finanziert wurden. Bei den Befragungen handelte es sich um Experteninterviews mit Leitfaden, deren Grundlage folgende Forschungsfragen waren:

Wie wirken sich die veränderten politischen Rahmenbedingungen auf den Rekrutierungspfad des Erbabgeordneten aus? Welche Veränderungen auf der Nachfrage- bzw. der Angebotsseite hat es gegeben? Ist seitens der befragten Erbabgeordneten eine Erbfolge geplant? Wie wird die Zukunft der Erbfolge von den Abgeordneten eingeschätzt?

Im Fall der vorliegenden Fragestellung boten sich qualitative Interviews an, da vor allem die subjektiven Sichtweisen und Motivationen der sozialen Akteure erforscht werden sollten. Darüber hinaus eignen sich qualitative Interviews besonders bei Untersuchungen von relativ unerforschten Bereichen. ${ }^{52}$ Da das Ziel einer Befragung Analyse und Vergleich der Inhalte sind, wurden halbstandardisierte Interviews bzw. Leitfadeninterviews geführt. Dabei wird ein Fragebogen als Leitfaden verwendet, dessen vorformulierte Fragen sich in Schlüsselfragen und in Eventualfragen, die je nach Interviewverlauf gestellt und variiert werden, gliedern.

Der Vorteil dieser Methode ist, dass sich trotz offener Antwortkategorien teilstrukturierte Befragungen durchführen lassen, die eine Vergleichbarkeit der Interviews gewährleisten. Die Abfolge der Fragen lässt sich jedoch sehr flexibel gestalten; sich im Gespräch ergebende Themen können aufgenommen und vertieft werden. ${ }^{53}$ Somit bildet diese Befragungsmethode einen guten Kompromiss zwischen der erwünschten Flexibilität und der Vergleichbarkeit der Interviews. Uwe Flick begründet die Tatsache, dass Leitfaden-Interviews eine wachsende Aufmerksamkeit erfahren, wie folgt: „Diese Aufmerksamkeit ist von der Erwartung bestimmt, dass in der relativ offenen Gestaltung der Interviewsituation die Sichtweisen des befragten Subjekts eher zur Geltung kommen als in den standardisierten Interviews oder Fragebögen [...]. “54

Im Fall der vorliegenden Untersuchung hat eine mündliche Befragung also einige Vorteile gegenüber einer Befragung per Fragebogen, bei der der Verlauf zwangsläufig standardisiert ist.

\footnotetext{
${ }^{52}$ Vgl.: Flick, Uwe; Kardoff, Ernst von; Steinke, Ines: Was ist qualitative Forschung? Einleitung und Überblick, in: Flick, Uwe; von Kardoff, Ernst; Steinke, Ines (Hg.): Qualitative Forschung. Ein Handbuch, Rowohlt, Reinbek 2000, S. 25; Flick, Uwe: Qualitative Forschung. Theorie, Methoden, Anwendung in Psychologie und Sozialwissenschaften, 4. Auflage, Rowohlt Verlag, Hamburg 1999.

${ }^{53}$ Vgl.. Atteslander, Peter: Methoden der empirischen Sozialforschung, 8. bearb. Auflage, de Gruyter, Berlin 1995, S. 161-163.

${ }^{54}$ Flick, Uwe: Qualitative Sozialforschung. Eine Einführung, Rowohlt Verlag, Hamburg 2002, S. 117.
} 
Hinzu kommt, dass die Ausfallquote, d.h. die Zahl der nicht zurückgeschickten Fragebögen, kaum vorherzusehen und schwer zu kontrollieren wäre. ${ }^{55}$

Aus den oben genannten Forschungsfragen wurde ein Fragebogen entwickelt, der sich im Anhang befindet. Dieser Fragebogen gliedert sich in fünf Teile. Der erste Teil besteht aus der Einstiegfrage nach dem persönlichen Einstieg in die Politik und der Frage nach dem Druck von Außen (Partei, Familie etc.). Der zweite Teil behandelt die Situation der Erbabgeordneten unter dem 1955er-System und die Gründe für die hohe Zahl von Erbabgeordneten. Im dritten Teil sollten die Erbabgeordneten die Veränderungen nach den politischen Reformen erläutern. Im vierten Teil wurden die Erbabgeordneten nach der eigenen Nachfolgeregelung befragt, bzw. nach den Chancen, ihren eigenen Wahlkreis weitergeben zu können. Zum Abschluss des Interviews sollten sich die Politiker schließlich allgemein zu den Zukunftschancen dieses Rekrutierungspfades äußern.

Der Fragebogen der zweiten Befragung im Frühjahr 2005 wurde leicht verändert und verbessert. So gab es zwei Kategorien, die zwei Jahre zuvor nicht berücksichtigt worden waren und sich erst aus der Befragung 2003 ergaben. Dabei handelte es sich zum einen um die Bedeutung der Erziehung, des „Hineinwachsens in die Politik“, für die Entscheidung, in die Politik zu gehen. Zum anderen wurde von vielen Abgeordneten das Problem der Abwanderung von „potentials“, von qualifizierten Kandidaten aus der LDP in andere Oppositionsparteien angesprochen. Diese Aspekte wurden daher bei den Fragen im Frühjahr 2005 stärker thematisiert, ein Fragekomplex über die Probleme bei der Kandidatenrekrutierung zusätzlich in den Fragebogen aufgenommen. Auf die einzelnen Kategorien wird aber später noch genauer eingegangen.

Der Einsatz qualitativer Forschung, also in diesem Fall qualitativer Interviews, ist in diesem Bereich nicht ungewöhnlich. Werner J. Patzelt beispielsweise führte die 1995 veröffentlichte Studie „Abgeordnete und ihr Beruf“ durch. 54 Abgeordnete auf Landes-, Bundes- und Europaebene wurden in qualitativen Interviews unter anderem zu ihrem Amtsverständnis, ihrer Parlaments- und Wahlkreisarbeit und zur politischen Rekrutierung befragt. Die Frage nach der Rekrutierung von Abgeordneten wurde, ähnlich wie in der vorliegenden Arbeit, auch unter dem Aspekt untersucht, aus welchen Motiven sich Abgeordnete entschließen, die Politik zu ihrem Beruf zu machen. ${ }^{56}$

Auch Park Cheol Hee befragte im Rahmen seiner bereits erwähnten Studie einige Politiker und betrachtete die Auswirkungen der Reformen in Tôkyôter Wahlkreisen. ${ }^{57}$ Die Untersuchung behandelt zwar vor allem die Wahlkampfstrategien und Prozesse im Wahlkreis selbst. Auf po-

\footnotetext{
${ }^{55}$ Vgl.: Patzelt, Werner J.: A.a.O., S. 283.

${ }^{56}$ Vgl.: Ebd., S. 265 ff.

${ }^{57}$ Vgl.: Park, Cheol Hee: a.a.O., S. $204 f$.
} 
litische Rekrutierung wird an einigen Stellen jedoch ebenfalls eingegangen. Für die Anwendung qualitativer Interviews in der Forschung über Abgeordnete gibt es also sowohl in Deutschland als auch in Japan Beispiele.

Ellis S. Krauss und Robert Pekkanen, die in einer Untersuchung zu den Auswirkungen der politischen Reformen dieselbe Methode benutzen, kritisieren, dass (qualitative) Interviews so zurückhaltend eingesetzt werden: „Part of the problem has been, that much of the theory of how electoral systems affect politics is based on explicit or implicit assumptions about the incentives of politicians and parties, yet few if any studies have actually asked the politicians themselves whether and how their behavior has changed and why." 58

\subsubsection{Auswahl der Interviewpartner}

Bei qualitativen Interviews werden die Interviewpartner nicht nach Repräsentativität ausgesucht, sondern danach, ob sie als „typischer Fall“ gelten können (theoretical sampling). Allerdings sollte der Interviewer auch für die von den eigenen Vorstellungen abweichenden Fälle offen sein. ${ }^{59}$ Gerade unter den Abgeordneten im japanischen Parlament gibt es sehr viele verschiedene „Typen“ von Erbabgeordneten. Dieser Tatsache musste bei der Auswahl Rechnung getragen werden.

Als Interviewpartner wurden Abgeordnete ausgewählt, deren Familien mindestens in zweiter Generation in der nationalen Politik aktiv sind und die über ein Mandat im Unterhaus verfügen. Da sich die Fragestellung vor allem auf Veränderungen der politischen Strukturen bezieht, wurden vor allem Erbabgeordnete befragt, die schon vor den politischen Reformen in der Politik tätig waren (als Abgeordneter, Sekretär etc.).

Ein weiteres Auswahlkriterium war, dass die ausgewählten Erbabgeordneten über ein Direktmandat in das Parlament eingezogen waren, da sich die Fragen vor allem auf die Veränderungen in den Wahlkreisen und die Form der Nachfolgeregelung bezogen. Die Fragen der Interviews konzentrierten sich also auf die Verhältnisse in den Direktwahlkreisen.

Da das Phänomen der Erbabgeordneten vor allem ein Phänomen der Liberaldemokratischen Partei unter dem 1955er-System war und auch heute noch die meisten Erbabgeordneten in der LDP bzw. in deren Nachfolgeparteien zu finden sind, wurde die Auswahl der Interviewpartner unter den Erbabgeordneten der LDP bzw. der teilweise aus der LDP hervorgegangenen DPJ als größte Oppositionspartei getroffen.

\footnotetext{
${ }^{58}$ Krauss, Ellis S.: Pekkanen, Robert: Explaining Party Adaption to Electoral Reform. The Discreet Charme of the LDP? In: Journal of Japanese Studies, Vol. 30, No. 1/2004, S. 9.

${ }^{59}$ Vgl.: Lamnek, Siegfried: Qualitative Sozialforschung. Bd. 2: Methoden und Techniken, Psychologie Verlags Union, München 1989, S. 93.
} 
In der folgenden Tabelle sind die 14 Interviewpartner, darunter 12 Erbabgeordnete im engeren Sinne (s.u.), aufgeführt, die 2003 und 2005 befragt wurden. Zu jener Zeit befanden sich 136 Erbabgeordnete im japanischen Unterhaus, das heißt, dass 9\% der Erbabgeordneten befragt wurden. Im Anhang findet sich eine gesonderte Aufstellung der Abgeordneten mit biographischen Angaben.

\section{Abbildung 3: Liste der befragten Abgeordneten}

\begin{tabular}{|c|c|c|c|c|}
\hline & Name & Partei & Wahlkreis & Interview \\
\hline 1 & Ôno Yoshinori & LDP & Kagawa 3 & 2003 \\
\hline 2 & Matsuno Yorihisa & DPJ & Kumamoto 1 & 2003 \\
\hline 3 & Watanabe Shû & DPJ & Shizuoka 6 & 2003 \\
\hline 4 & Usui Hideo & LDP & Chiba 1 & 2003 \\
\hline 5 & Itô Shintarô & LDP & Miyagi 4 & 2003 \\
\hline 6 & Hamada Yasukazu & LDP & Chiba 12 & 2005 \\
\hline 7 & Harada Yoshitsugu & LDP & Shizuoka 2 & 2005 \\
\hline 8 & Hori Kôsuke & LDP & Saga 3 & 2005 \\
\hline 9 & Funada Hajime & LDP & Tochigi 1 & 2005 \\
\hline 10 & Kosaka Kenji & LDP & Nagano 1 & 2005 \\
\hline 11 & Kômura Masahiko & LDP & Yamaguchi 1 & 2005 \\
\hline 12 & Ishiba Shigeru & LDP & Tottori 1 & 2005 \\
\hline 13 & Terata Manabu & $D P J$ & Akita 1 & 2005 \\
\hline 14 & Usui Shôichi & $L D P$ & Präfekturparlament & 2005 \\
\hline
\end{tabular}

Zwei Interviewpartner, der Abgeordnete Terata Manabu und der Präfekturabgeordnete Usui Shoichi, die im Frühjahr 2005 befragt wurden, stellten besondere Fälle dar. Terata Manabu hatte im Gegensatz zu den anderen Kandidaten keinen Verwandten, der vor ihm auf nationaler Ebene tätig war - sein Vater war Gouverneur der Präfektur Akita. Deshalb kann man ihn nur als Erbabgeordneten im weitesten Sinn bezeichnen. ${ }^{60}$ Auch sein Alter, der Abgeordnete Terata

\footnotetext{
${ }^{60}$ Auf die Begriffsproblematik wird später noch genauer eingegangen.
} 
war zum Zeitpunkt der Befragung erst 28 Jahre alt, unterschied ihn von den anderen befragten Politikern. Dennoch stellte Terata Manabu einen interessanten Fall bzw. Interviewpartner dar, da er sich in seiner Partei, der Demokratischen Partei, mit dem Generationswechsel und politischer Rekrutierung beschäftigt.

Auch Usui Shôichi ist kein Erbabgeordneten im engeren Sinn, da er als Abgeordneter des Parlaments der Präfektur Chiba nur auf regionaler Ebene tätig ist. Da er aber für das Parlament kandidierte, als sein Vater noch Abgeordneter der Unterhauses war, ist er ebenfalls ein interessanter Fall. Hier lässt sich ein Generationswechsel sehr gut beobachten.

Die in Frage kommenden Abgeordneten wurden mit Hilfe einer Liste von Erbabgeordneten in dem Nachschlagewerk Seikan Yôran ${ }^{61}$ sowie den dort angegebenen biographischen Daten ausgewählt. Über E-Mail und Fax wurde Kontakt zu den Abgeordneten aufgenommen. Einige Interviews wurden durch Bekannte, die teilweise selbst in der Politik aktiv sind, vermittelt.

\section{Abbildung 4: Wahlkreise der befragten Abgeordneten}

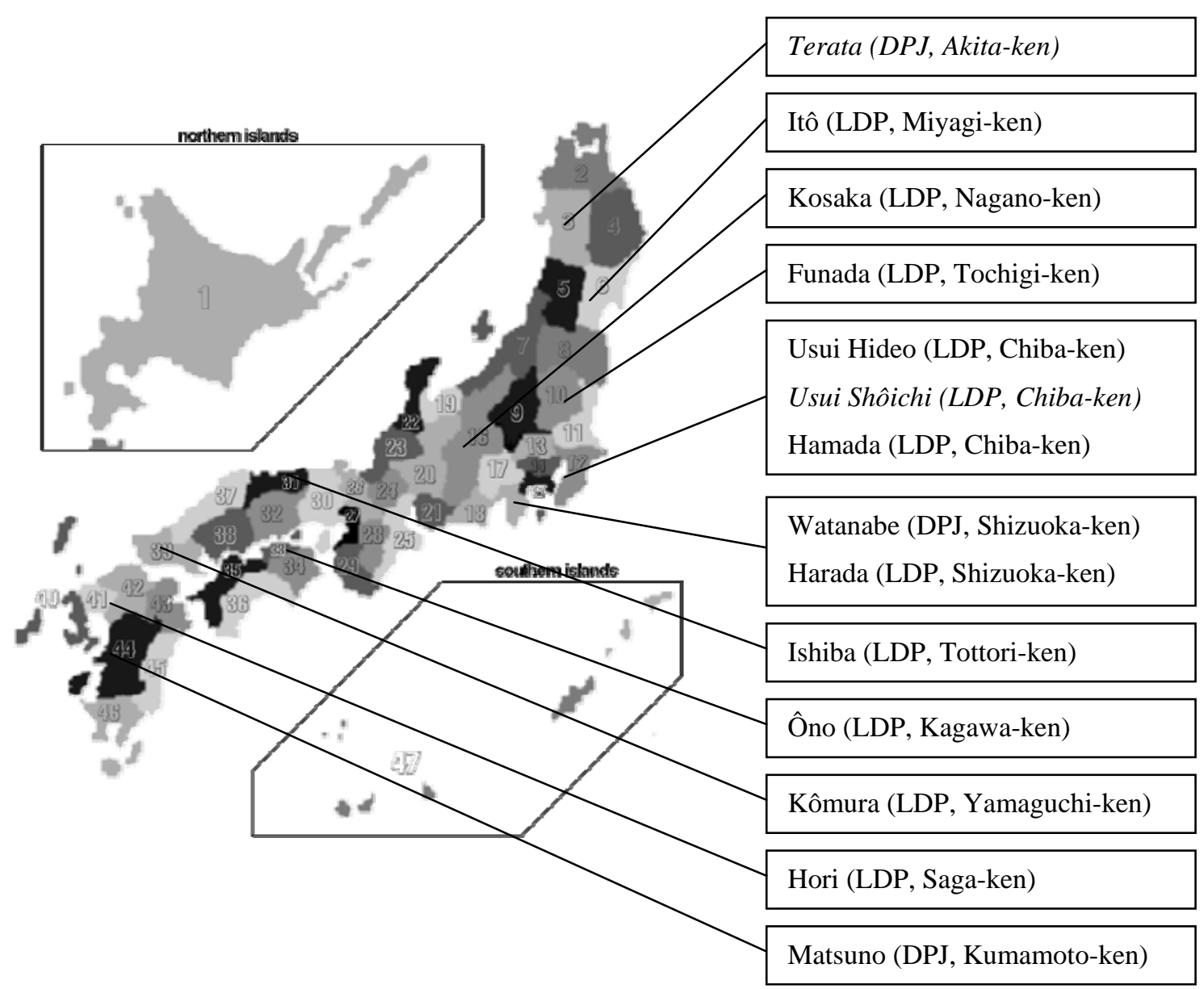

${ }^{61}$ Saitô, Keiko: Seikanyôran, Vol. 20, No. 47, a.a.O., Sonderbeilage, S. 2 ff. 
Die Frage nach den Gründen für Zu- oder Absagen ist von nicht unerheblicher Bedeutung. Eine wichtige Rolle spielte sicherlich, ob sich die jeweiligen Abgeordneten selbst als Erbabgeordnete definieren oder nicht. Aber selbst bei den befragten Politikern fanden sich höchst unterschiedliche Selbstbilder, wie später noch gezeigt wird. Das Alter dagegen spielte keine Rolle, da, mit Ausnahmen, Interviewpartner ausgesucht wurden, die bereits älter waren und über längere Erfahrungen in der Politik verfügten. Auch die Position der Abgeordneten in ihrer Partei oder der Regierung waren wohl kein Grund für Zu- oder Absagen, da sich unter den Interviewpartnern sowohl so genannte „Hinterbänkler“ befanden als auch Führungspersonen der LDP oder der Regierung. Bezeichnend ist allerdings, dass fast alle der befragten Politiker über internationale Erfahrungen verfügten. Einige waren vor ihrer politischen Karriere längere Zeit im Ausland gewesen, etwa als Angestellte einer japanischen Firma. Andere hatten durch ein politisches Amt als Minister etc. zahlreiche internationale Kontakte.

Die Wahlkreise der befragten Abgeordneten liegen in verschiedenen Regionen Japans, die vor allem ländlich geprägt sind (Miyagi, Kagawa, Shizuoka, Kumamoto) oder zum Einzugsgebiet der Metropole Tôkyô gehörten (Chiba). Insofern kann die Auswahl als typisch bezeichnet werden, da die Erbfolge, wie noch gezeigt wird, während des 1955er- Systems vor allem ein Phänomen der ländlichen oder suburbanen Wahlkreise war. Diese waren nämlich zumeist auch LDP-Hochburgen.

Eine weitere Variante der qualitativen Befragung ist das Experteninterview. Dabei ist der Befragte Experte auf einem bestimmten Feld, in diesem Fall also für politische Rekrutierung. ${ }^{62}$ Neben den Leitfadeninterviews mit japanischen Erbabgeordneten wurden zwei Interviews mit Shiratori Rei, Professor für Politik und Leiter des Politikforschungsinstituts in Tôkyô, und Ishikawa Akimasa, Sprecher der Wahlkampfzentrale (senkyo taisaku honbu) der LDP, geführt. Diese beiden Interviews dienten jedoch primär zum Verständnis des gesammelten Materials und dem Sammeln von Zusatzinformationen. Sie sind deshalb nicht gesondert aufgeführt.

\subsubsection{Durchführung der Interviews und Auswertung}

Die Gespräche dauerten in der Regel etwa 45 Minuten. Die Interviews wurden bis auf das Interview mit Usui Hideo, das in seinem Haus in Chiba stattfand, in den Abgeordnetenbüros in Tôkyô durchgeführt und mit einem Tonband aufgenommen (Datenerhebung), anschließend transkribiert und ins Deutsche übersetzt (Datenerfassung). Schließlich wurden die Rohfassungen noch einmal mit den Tonbandaufzeichnungen abgeglichen.

Bei den geführten Interviews spielte die Alltagssituation, wie es häufig der Fall ist, keine Rol-

\footnotetext{
${ }^{62}$ Vgl.: Flick, Uwe: Qualitative Forschung. Theorie, Methoden, Anwendung in Psychologie und Sozialwissenschaften, a.a.O., S. 109-110.
} 
le, da Politiker in der Regel an Interviewsituationen gewöhnt sind. Drei der Befragten hatten außerdem vor ihrer politischen Karriere als Journalisten gearbeitet. An dieser Stelle sollte man deshalb berücksichtigen, dass sich die Befragten in Interviews möglichst gut darstellen möchten, da für Berufspolitiker eine positive Selbstdarstellung und Präsentation nach außen von großer Bedeutung ist.

Wenn sich beispielsweise ein Interviewpartner in einem Gespräch klar für politische Reformen und transparente Auswahlmechanismen ausspricht, muss das nicht zwangsläufig bedeuten, dass er diese Meinung auch innerhalb der Partei so deutlich vertritt. Dieser wichtige Punkt muss bei der Auswertung berücksichtigt werden. Wie offen sich die Interviewpartner gegenüber einem japanischen Wissenschaftler geäußert hätten, ist eine andere Frage, der aber hier nicht weiter nachgegangen werden kann. Werner J. Patzelt, der sich ähnlicher Kritik ausgesetzt sah, bemerkte dazu, dass es „sowieso keine sinnvolle Alternative dazu [gibt], Parlamentarier persönlich zu befragen, wenn man etwas über ihr Amtsverständnis und über die Prozesse ihrer persönlichen Meinungs- oder Willensbildung erfahren will: nur sie können darüber authentisch Auskunft geben. “63

Die Interviews wurden bei der Übersetzung sprachlich etwas geglättet, vor allem um verbale Vieldeutigkeiten von Redewendungen etc. besser verständlich zu machen. Regionale sprachliche Besonderheiten, Dialekte etc. lassen sich in der Übersetzung dagegen nur schwer darstellen und sind deshalb nicht erfasst. Dennoch wurde versucht, die besondere Klangfarbe der Interviews zu erhalten. ${ }^{64}$ Die Interviews wurden auch nicht, wie es häufig der Fall ist, anonymisiert. Aufgrund ihrer exponierten Stellung wären die Interviewten auch bei einer Anonymisierung leicht zu finden. Hinzu kommt, dass einige Antworten der befragten Abgeordneten erst durch die biographischen Hintergründe verständlich werden, die im Anhang aufgeführt sind.

Ebenfalls musste bedacht werden, dass es bei der Übersetzung, trotz der Hilfe von Muttersprachlern, bei Fachtermini, Sprichwörtern etc. erhebliche kulturelle Bedeutungsunterschiede und verbale Vieldeutigkeiten gibt. Bestes Beispiel ist wahrscheinlich der Begriff des Erbabgeordneten selbst.

Zwar wird unter 2.3 eine Definition vorgenommen, die der Definition in der japanischen Fachliteratur entspricht (bzw. aus der japanischen Fachliteratur übernommen wurde). Dennoch lehnten einige Abgeordnete den Begriff des Erbabgeordneten für sich ab, obwohl sie den Wahlkreis bzw. die Basisorganisation ihres Vorgängers übernommen hatten und somit eindeutig der Definition des Erbabgeordneten entsprachen. Dies wurde auch als Grund für einige Absagen

\footnotetext{
${ }^{63}$ Patzelt, Werner J.: a.a.O., S. 13.

${ }^{64}$ Vgl.: Ebd., S. 332.
} 
genannt. Die Definition des Wortes „Erbabgeordneter“ wird an anderer Stelle noch genauer besprochen.

Für die Bestimmung der Auswertungskategorien gibt es verschiedene Möglichkeiten. Eine Möglichkeit ist, die Kategorien aus dem Material heraus zu entwickeln (induktiv). Man kann die Kategorien aber auch außerhalb des Materials, z.B. aus dem Interviewleitfaden direkt gewinnen (deduktiv). ${ }^{65}$ Im Fall der vorliegenden Arbeit wurden die Kategorien aus dem Leitfaden übernommen, wie es Udo Kuckartz empfiehlt: „Wenn man schon mit einem Leitfaden arbeitet, bietet es sich an, Kategorien in direkter Anlehnung an diesen zu definieren. “66

Während der Auswertung des Materials tauchten auch Themen auf, die im Leitfaden (2003) nicht bedacht wurden, wie etwa das Selbstbild der Erbabgeordneten und die Abwanderung von Kandidaten von der LDP zur DPJ. Da diese Aspekte für die Fragestellung sehr wichtig erschienen, wurden sie ebenfalls als Kategorien übernommen. Hier zeigte sich der Vorteil der Offenheit halbstandardisierter Interviews. Das Codieren des Materials erfolgte mit Hilfe von winMax Pro 2000®, einem Textanalyse-System für die Sozialwissenschaften.

Im Folgenden sollen für die Darstellung der Ergebnisse die Textstellen in den Interviews als illustrierende Zitate benutzt werden. Die Anordnung der Interviewpassagen richtet sich in einer solchen Arbeit vor allem nach einer analytisch sinnvollen Gliederung. Auch sind zu kurze und verstümmelte Textpassagen kaum nützlich für die Analyse. Außerdem kommt es vor, dass einzelne Textstücke für verschiedene Kategorien von Bedeutung sind.

Im Anhang finden sich die biographischen Besonderheiten der befragten Abgeordneten, die bei der Auswertung wichtige Zusatzinformationen darstellen. ${ }^{67}$ Zwei Interviews (Ôno Yoshinori, Funada Hajime) sind als Beispiele in voller Länge im Anhang aufgeführt.

\footnotetext{
${ }^{65}$ Vgl.: Kuckartz, Udo: Computergestützte Analyse qualitativer Daten: Eine Einführung in Methoden und Arbeitstechniken, Westdeutscher Verlag, Opladen 1999, S. $204 f$.

${ }^{66}$ Ebd., S. 209.

${ }^{67}$ Vgl.. Lamnek, Siegfried: A.a.O., S. 99, 105.
} 


\section{Erbabgeordnete: Entstehung und Karrierepfade}

\subsection{Die Opportunitätsstruktur}

Die Entstehung des Rekrutierungspfades des Erbabgeordneten in Japan ist wie bereits erwähnt eng mit dem politischen System in der Zeit von 1955 bis 1993 verbunden. Dieser Zeitraum wird in Japan als 1955er-System bezeichnet. Das Verständnis dieser Zeit und die Darstellung einiger Grundbegriffe sind Voraussetzung für die Analyse des Phänomens der Erbabgeordneten. Im Folgenden sollen nun die institutionellen Rahmenbedingungen des 1955erSystems dargestellt werden, soweit sie für die Analyse der politischen Rekrutierung von Bedeutung sind, um dann anschließend anhand einer Analyse der Angebots- und Nachfrage die hohe Zahl von Erbabgeordneten zu erklären.

\subsubsection{Das 1955er-System}

\subsubsection{Die „Eineinhalb-Parteien-Herrschaft“ der LDP}

Das Zeitalter des 1955er-Systems begann mit dem Zusammenschluss der Liberalen Partei und der Demokratischen Partei. Dieser Zusammenschluss war eine Reaktion auf die Vereinigung des lange gespaltenen linken und rechten Flügels der japanischen Sozialisten zur SPJ (Sozialistische Partei Japans). So entstand eine Art „Eineinhalb-Parteien-System“ “68 in dem die LDP praktisch allein regierte und die SPJ die größte Oppositionspartei darstellte. Dieses 38 Jahre währende System garantierte auf der einen Seite die für den wirtschaftlichen Aufstieg Japans wichtige Stabilität, erwies sich aber andererseits als äußerst korruptionsanfällig. ${ }^{69}$ Kritik wurde dabei nicht nur an der Alleinherrschaft der LDP geübt, sondern auch an der SPJ, die in den Augen ihrer Kritiker keine wirksame Opposition darstellte. ${ }^{70}$

Die Nachkriegszeit bis Mitte der 1950er Jahre war in Japan von heftigen ideologischen Auseinandersetzungen, Massenprotesten und einem „chaotischen“ Mehrparteiensystem geprägt gewesen. ${ }^{71}$ Eine SPJ-Regierung war mit ihrem marxistischen Programm gescheitert und die kommunistische Partei hatte sich durch gewalttätige Massenkundgebungen diskreditiert. Diese

\footnotetext{
${ }^{68}$ Bruns, Gabriele: Die Japanische Demokratie. Kieler Schriften zur politischen Wissenschaft 10, Frankfurt/M., 1999, S. 79.

„Eineinhalb-Parteiensystem“ wird bei Bruns definiert als System, „, in dem eine dominante Partei stets die Regierungsmacht innehat, während zwar andere Parteien existieren, aber zu keinem Zeitpunkt in der Lage sind, die Regierungsverantwortung zu übernehmen. “

${ }^{69}$ Vgl.: Okada, Kenji: Seiji Seido no Shikumi, (The mechanism of the political system), Tokyo 1999, S. $130 \mathrm{ff}$.

${ }^{70}$ Bruns, Gabriele: A.a.O., S. 93-110.

${ }^{71}$ Vgl.: Ishida, Takeshi; Krauss, Ellis S.: Democracy in Japan: Issues and Questions, in: Ishida, Takeshi; Krauss, Ellis S. (Hg.): Democracy in Japan, 3.Auflage, University of Pittsburgh Press, Pittsburgh 1994, S. 24.
} 
dienten als willkommener Anlass, kommunistische Aktivisten zu verfolgen. Aus diesen Gründen kam den konservativen Kräften die Führungsrolle Japans zu. ${ }^{72}$

Die Anfang der 1950er Jahre von Ministerpräsident Yoshida Shigeru entwickelte hoshu honryû (conservative policy line) basierte auf der Zusammenarbeit zwischen konservativen Kräften und der Bürokratie. Yoshida hatte eine Reihe von ehemaligen Angehörigen der Ministerialbürokratie für seine Partei angeworben, die bald in Regierungspositionen gelangten. ${ }^{73}$

Ein weiteres Element dieser Politik bildeten die Interessenverbände. Wegen des Mangels an lokalen Parteiorganisationen bemühten sich viele konservative Politiker in ihren Wahlkreisen um die Unterstützung z.B. landwirtschaftlicher Genossenschaften. Auf nationaler Ebene war die LDP eng mit dem big business verflochten. ${ }^{74}$ Diese Verbindung aus Wirtschaft (Interessenverbänden), Bürokratie und Politik bildete das so genannte Iron Triangle, das im nächsten Abschnitt beschrieben wird.

Im Rahmen des National Income-Doubling Plan wurden sämtliche Kräfte des Landes auf den wirtschaftlichen Aufbau Japans konzentriert. Auseinandersetzungen mit der Opposition wurden durch die Aufnahme und Einbindung oppositioneller Forderungen entschärft. Infolge des wirtschaftlichen Aufschwungs bildete sich bald eine wirtschaftlich und sozial stabile „New Middle Mass-Gesellschaft“ heraus, die die Basis für die LDP und ihre Politik darstellte. ${ }^{75}$ Das enorme Wirtschaftswachstum brachte jedoch auch Probleme wie Umweltverschmutzung, Landflucht oder Überbevölkerung der Städte mit sich. Vor allem lokal agierende Bürgerbewegungen nahmen sich der neuen politischen Themen an. ${ }^{76}$ „The 1960 s and 1970 s saw a more general broadening of interest from narrowly defined goals of economic growth to a greater concern with quality of life - not just of the environment but in Japanese society as well. "77 Diese zunehmende Pluralisierung spiegelte sich auch in der LDP wieder. Als Reaktion auf neue politische Gruppen versuchte die LDP sich zu öffnen. Sie fand sich allerdings bald in der Situation, zu vielen, oft widersprüchlichen, Interessen gerecht werden zu müssen: „The LDP has become a friend of everybody, but a special friend of nobody. “78.

\footnotetext{
${ }^{72}$ Muramatsu, Michio; Krauss, Ellis S.: The Conservative Policy Line and the Development of Patterned Pluralism, in: Yamamura Kozo; Yasuba Yasukichi (Hg.): The Political Economy of Japan. Volume 1. The Domestic Transformation, Stanford University Press, Stanford 1987, S. 519-520.

${ }^{73}$ Vgl.: Muramatsu, Michio; Krauss, Ellis S.: A.a.O., S. 520-522.

${ }^{74}$ Diese Konglomerate entsprachen den zaibatsu der Vorkriegszeit, die von der Besatzungsmacht weitgehend zerschlagen wurde, sich aber nun neu gruppierten und deren Herzstück oft Großbanken waren. Vgl.: Beasley, W.G.: The Rise of Modern Japan, St. Martin’s Press, New York 1990, S. 245.

${ }^{75}$ Vgl.: Muramatsu, Michio; Krauss, Ellis S.: A.a.O., S. 523-526.

${ }^{76}$ MacDougall, Terry E.: Democracy and Local Government in Postwar Japan, in: Ishida, Takeshi; Krauss, Ellis S. (Hg.): Democracy in Japan, 3.Auflage, University of Pittsburgh Press, Pittsburgh 1994, S. 151.

77 Campbell, John Creighton: Democracy and Bureaucracy in Japan, in: Ishida, Takeshi; Krauss, Ellis S. (Hg.): Democracy in Japan, 3.Auflage, University of Pittsburgh Press, Pittsburgh 1994, S. 124.

${ }^{78}$ Zitiert in: Blechinger, Verena: Politik und Familienbande, a.a.O., S. 179.
} 
Die SPJ bekam diese Entwicklung ebenfalls zu spüren: Neue Oppositionsparteien etablierten sich, wie etwa die religiöse Kômeitô (Clean Government Party - CGP). Die Kômeitô entstand 1961 als politischer Arm der buddhistischen Laiensekte Sôka Gakkai. Die 1930 von Makiguchi Tsunesaburô gegründete Bewegung geht auf die relativ nationalistische Sekte Nichiren Shôshû zurück und wurde während des Krieges von der Militärregierung verfolgt. ${ }^{79}$ Sie galt während des japanischen Wirtschaftsbooms nach dem 2. Weltkrieg als Stimme der „Zukurzgekommenen“ und weist sehr geschlossene und relative strikte Gruppenstrukturen auf. Ihre Missionierungsmethoden werden mitunter als sehr aggressiv beschrieben. Unter ihrem Präsidenten Ikeda Daisaku, der immer noch als eine Art Übervater gilt, wuchs die Sekte von 70.000 Mitgliedern im Jahr 1953 auf 7 Mio. Mitglieder im Jahr 1969. Das entsprach zum damaligen Zeitpunkt etwa 7\% der Bevölkerung. ${ }^{80}$ Heute hat die Sekte nach eigenen Angaben über 12 Mio. Mitglieder in 190 Staaten, davon 10 Mio. in Japan (, was ca. 8,5\% der Bevölkerung entsprechen würde).

Die Anhängerschaft der Kômeitô galt bisher als formal schlechter gebildet als die anderer Parteien. Hingegen war der Bildungsstand der KPJ-Wähler in den 1970er Jahren, vermutlich aufgrund der großen Anhängerschaft unter Akademikern, von allen Parteien am höchsten. ${ }^{81}$

Gemäß dem Ziel der Sôka Gakkai, Politik und Buddhismus zu vereinen (ôbutsu-myôgô), propagierte die Kômeitô einen „Menschlichen Sozialismus“ (ningensei shakaishugi). Auch gegen die Korruption der etablierten Parteien zog die „Partei für eine saubere Politik“ in den Wahlkampf. $^{82}$

Die SPJ büßte in dieser Zeit einen erheblichen Teil ihrer Parlamentssitze ein und die Entstehung einer zweiten gemäßigten sozialistischen Partei (DSP - Demokratisch-Sozialistische Partei) dokumentierte darüber hinaus die Zersplitterung der japanischen Gewerkschaften in einen radikaleren (Sôhyô, SPJ-nah) und einen gemäßigteren Flügel (Dômei, DSP-nah), der vor allem aus Unternehmensgewerkschaften der großen Konzerne bestand. ${ }^{83}$

Die so genannten third parties ${ }^{84}$ konnten erstmals 1967 mehr als 10\% der Stimmen erringen und ihr Stimmenanteil pendelte sich nach 1976 bei 20 bis 30\% ein, so dass man für die Zeit ab den 1970er Jahren kaum noch von einem Eineinhalb-Parteien-System im eigentlichen Sinn sprechen kann. „The JSP's share might have declined, but with the addition of various third parties to its share, the total opposition vote and parliamentary strength reached virtual parity with the LDP. Although this change made the LDP's steering of the Diet more difficult, the

\footnotetext{
${ }^{79}$ Vgl.: White, James W.: The Sokagakkai and Mass Society, Stanford University Press, Stanford 1970, S. 1-3; 41.

${ }^{80}$ Vgl.: Ebd., S. 20-41.

${ }^{81}$ Vgl.: Ebd., S. 141.

${ }^{82}$ Vgl.: Ebd., S. 126-131.

${ }^{83}$ Vgl.: Seifert, Wolfgang: Gewerkschaften in der japanischen Politik von 1970 bis 1990 . Der dritte Partner? Westdeutscher Verlag, Opladen 1997, S. 84.

${ }^{84}$ Masumi, Junnosuke: Contemporary Politics in Japan, University of California Press, Berkeley 1995, S. 402.
} 
opposition parties were not in a position to coalesce and demand control of the government. “ 85 Die Oppositionsparteien wurden trotz ihrer relativen Schwäche in den politischen Entscheidungsprozess eingebunden. Ihre Zustimmung wurde durch Zugeständnisse und Beteiligung an wichtigen Gesetzesvorhaben gesichert. ${ }^{86}$ Die Opposition konnte wichtige Gesetzesvorhaben zwar nicht verhindern, aber zumindest lange herauszögern: „, [...] Though the opposition parties could never muster enough strength to oust the government, they were masters in harassing it. " 87

\subsubsection{Iron Triangle: Das eiserne Dreieck}

Die 38 Jahre der LDP-Regierung hatten natürlich auch Folgen für das politische und wirtschaftliche System Japans. „Die Einparteienherrschaft, die sich über die lange Regierungszeit der LDP erstreckte, führte zu einer Struktur, in der Politik, Bürokratie und Geld miteinander verwuchsen [...] “88 Diese Verflechtung wird als Iron Triangle oder kangyôsei, bestehend aus Bürokratie, Wirtschaft und Politik, bezeichnet und galt als Symbol des 1955er-Systems.

Die LDP wurde auf nationaler Ebene hauptsächlich vom Dachverband der japanischen Wirtschaft, dem Keidanren, dem Ärzteverband und der Union der Landwirtschaftlichen Genossenschaften (Nôkyô) unterstützt. ${ }^{89}$ So ist es kein Zufall, dass die LDP stets eine protektionistische Agrarpolitik verfolgte und in den ländlichen Räumen ihre treuesten Anhänger fand. Die Landwirtschaftlichen Genossenschaften sind weit mehr als nur Interessenverbände, sie bilden einen wichtigen Teil der Infrastruktur in den ländlichen Gebieten. Schließlich spielen die Nôkyô auch für die Mobilisierung von (konservativen) Wählern eine wichtige Rolle. ${ }^{90}$ Freilich waren auch die anderen Parteien auf die Hilfe von Verbänden und Organisationen angewiesen, die während der Wahlkämpfe bei der politischen Finanzierung und der Stimmenwerbung behilflich waren.

Innerhalb der LDP gab es ab den 1970er Jahren so genannte zoku giin, erfahrene Abgeordnete, die „Spezialisten“ für bestimmte Fachgebiete waren und von den verschiedenen Interessenverbänden als Ansprechpartner und Vermittler genutzt wurden. Viele Interessenverbände und Unternehmen änderten durch die bereits genannte Pluralisierung der Interessen in der LDP ihr Spendenverhalten und gaben auf nationaler Ebene ihr Geld nicht mehr der Parteizentrale, sondern direkt an die zoku giin und einzelne Parteiführer. In den Wahlkreisen erwarteten die Vertreter der lokalen Wirtschaft konkrete Gegenleistungen für ihre Unterstützung, wie etwa die

\footnotetext{
${ }^{85}$ Masumi, Junnosuke: A.a.O., S. 402.

${ }^{86}$ Vgl.: Schlesinger, Jacob M.: Shadow Shoguns. The Rise and Fall of Japan's Postwar Political Machine, $2^{\text {nd }}$ Edition, Standford University Press, Stanford 1999, S. 129.

${ }^{87}$ Ebd., S. 129.

${ }^{88}$ Okada, Kenji: A.a.O., S. 130.

${ }^{89}$ Vgl.: Ebd., S. 132-133.

${ }^{90}$ Vgl.: Krauss, Ellis S.: Politics and the Policymaking Process, in: Ishida, Takeshi; Krauss, Ellis S. (Hg.): Democracy in Japan, 3.Auflage, University of Pittsburgh Press, Pittsburgh 1994, S. 55.
} 
Vermittlung öffentlicher Aufträge. So entwickelte sich eine give-and-take-Mentalität bei politischen Spenden, die vor allem an ihrem direkten Nutzen gemessen wurden. ${ }^{91}$

\section{Abbildung 5: Japanische Parteien und organisierte Interessen}

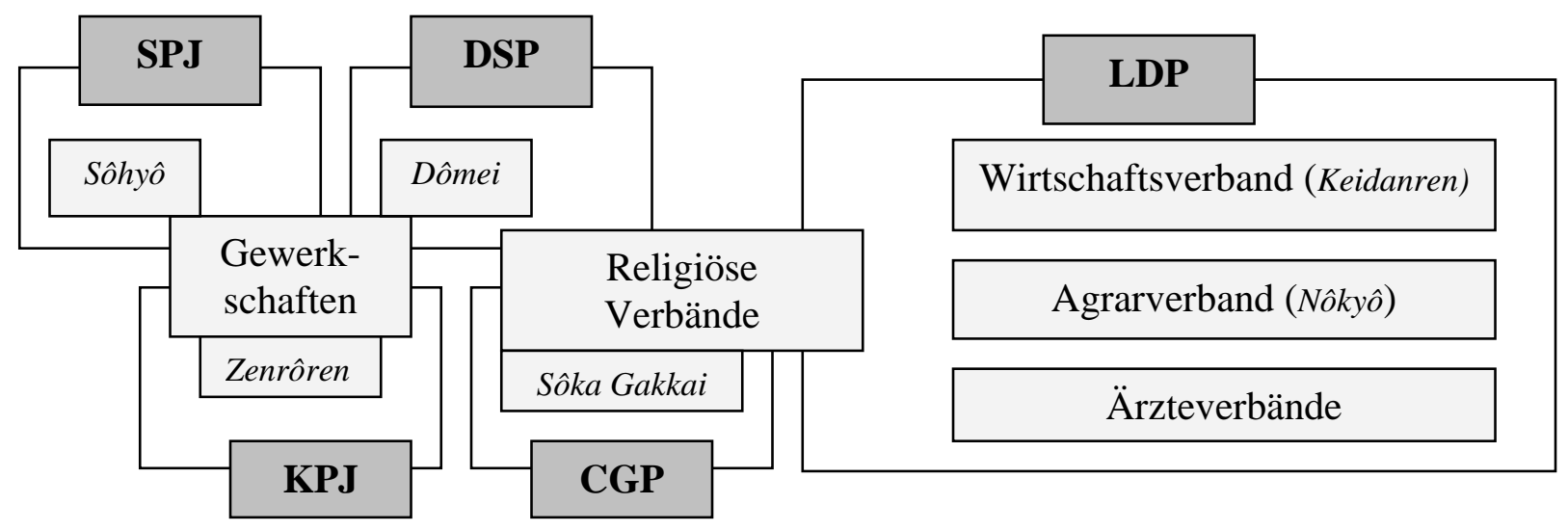

Nach: Okada, Kenji: Seijiseido-no Shikumi, Kabushikikaisha Nashime-sha, Tôkyô 1999, S. 132. (Okada, Kenji: Mechanismen des politischen Systems, Tôkyô 1999.)

Den dritten Teil des Iron Triangle bildete die Ministerialbürokratie, „[...] die den für das japanische Wirtschaftswachstum nach dem Zweiten Weltkrieg ausschlaggebenden Grundkonsens zwischen staatlichen Organen und Großindustrie herbeigeführt und damit letztlich auch das Fundament für die langjährige Einparteien-Herrschaft der LDP gelegt hat. “92 Ein Grund für die zunächst starke Stellung der Ministerialbürokratie findet sich in der Situation der Nachkriegszeit, als nach der Besetzung Japans die Bürokratie die einzige Konstante staatlicher Führung darstellte. Gegen viele Politiker der Vorkriegszeit waren Berufsverbote verhängt worden und in den politisch turbulenten 1950er Jahren fehlte darüber hinaus ein politisches Gegengewicht. Auch die LDP-Regierungen waren recht instabil. In 38 Jahren LDP-Regierung gab es immerhin 15 Kabinette. ${ }^{93}$ Die Gründe hierfür waren häufig innerparteiliche Machtkämpfe (s. 2.1.5).

Da politisch motivierte Neubesetzungen, wie sie bei einem Regierungswechsel häufig stattfinden, entfielen und sich die Rekrutierung des Personals auf wenige Elite-Universitäten ${ }^{94}$ kon-

\footnotetext{
${ }^{91}$ Vgl.: Blechinger, Verena: Politische Korruption in Japan, a.a.O., S. 179-181; Pohl, Manfred: Die politische Kultur Japans, in: Barloewen, Constantin von; Werhahn-Mees, Kai (Hg.): Japan und der Western. Band 3: Politik, Kultur und Gesellschaft, Frankfurt/M. 1986, S. 21. (Zur Situation in den Wahlkreisen s. 4.3. Das Wahlsystem)

${ }^{92}$ Zitiert nach: Blechinger, Verena: Politische Korruption in Japan, a.a.O., S. 186.

${ }^{93}$ Vgl.: Kagaya, Katsuo: Asahi Kîwâdo. Bessatsu Seiji - Kempô, Tôkyô 2000, S. 169-183.

${ }^{94}$ V.a. die staatlichen Universitäten Tôkyô und Kyôto, sowie die Privatuniversitäten Waseda und Keiô.
} 
zentrierte, galt die japanische Bürokratie als sehr homogen. ${ }^{95}$ Ein Großteil der Abgeordneten und Wirtschaftsführer hatte ebenfalls an einer der führenden Universitäten studiert, so dass Netzwerke (gakubatsu) zwischen Absolventen der gleichen Universität entstanden.

So existierte eine Reihe von formellen und informellen Beziehungen zwischen Bürokratie und Politik im Iron Triangle. Gesetzesvorlagen, die vom Kabinett in das Parlament eingebracht wurden, waren in der Regel vorher in den Ministerien ausgearbeitet worden. ${ }^{96}$ Bis in die 1970er Jahre wurde die Bürokratie als so übermächtig betrachtet, dass man sagte, die Parlamentsausschüsse seien lediglich „Sprecher der Verwaltungsabteilungen“997.

\section{Abbildung 6: Das Iron Triangle}

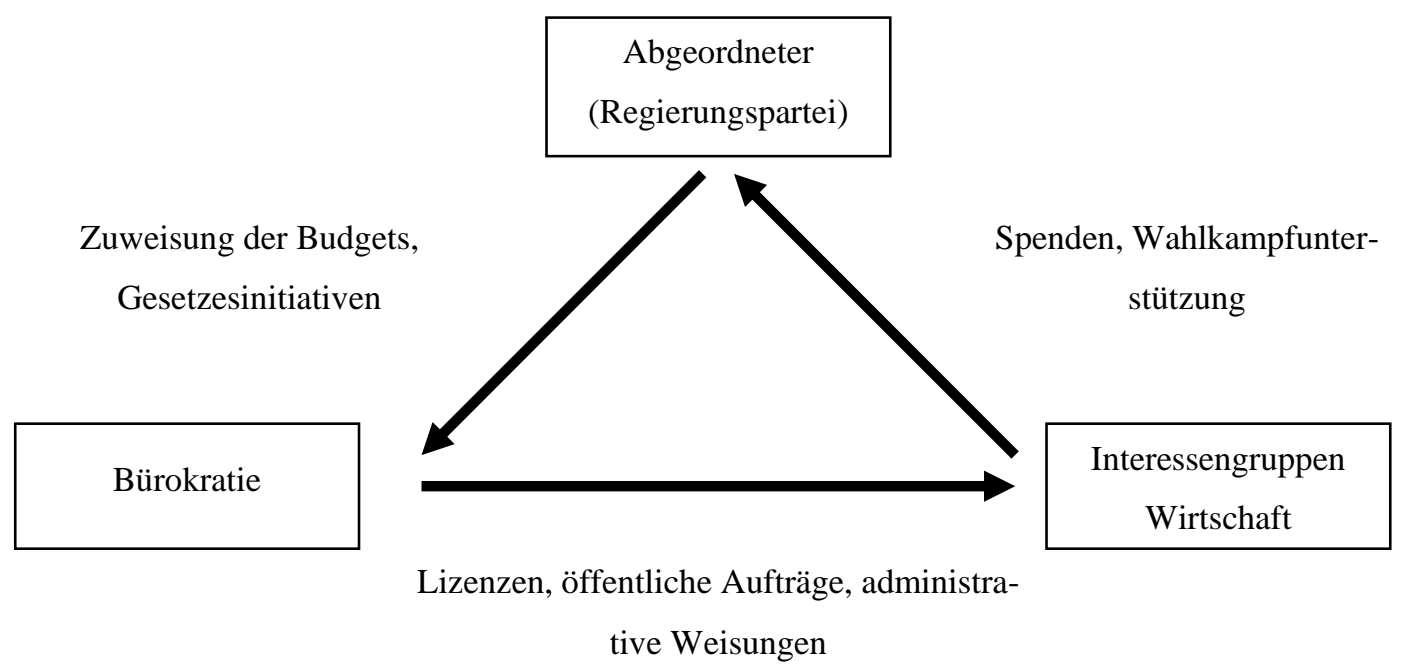

Nach: Okada, Kenji: Seijiseido-no Shikumi, Kabushikikaisha Nashime-sha, Tôkyô 1999, S. 133.

(Okada, Kenji: Mechanismen des politischen Systems, Tôkyô 1999.)

Die Beziehungen zwischen Bürokratie und Wirtschaft schlugen sich vor allem in den so genannten „Administrativen Weisungen“ (gyôsei shidô) nieder. Ein Großteil der Gesetzgebung beschränkte sich in Japan auf Rahmengesetze, die der administrativen Bürokratie einen breiten Spielraum ließen. Einzelnen Unternehmen wurden in vertraulichen Gesprächen bestimmte Auslegungen und Verhaltensweisen „nahegelegt““ ${ }^{98}$ Gleichzeitig gab es eine sehr restriktive Lizenzvergabe, die Unternehmen mit guten Kontakten zu den Ministerien nutzten, um Konkurrenz fernzuhalten. ${ }^{99}$ Als Gegenleistung für ihr Wohlverhalten wurden die zuständigen Beamten

\footnotetext{
${ }^{95}$ Vgl.: Pohl, Manfred: Die politische Kultur Japans, a.a.O., S. 24.

${ }^{96}$ Vgl.: Blechinger, Verena: Politische Korruption in Japan, S. 199.

${ }^{97}$ Pohl, Manfred: Die politische Kultur Japans, a.a.O., S. 25.

${ }^{98}$ Vgl.: Blechinger, Verena: Politische Korruption in Japan, a.a.O., S. $204 \mathrm{ff}$.

${ }^{99}$ Vgl.: Ebd., S. 208f.
} 
in teure Restaurants oder zu Golfwochenenden eingeladen. Größter Anreiz für die Beamten war jedoch eine mögliche zweite Karriere als amakudari. ${ }^{100}$

Viele japanische Beamte gingen nach ihrer Karriere, die oft schon mit 55 Jahren endete, in die Politik oder als amakudari („Vom Himmel Gestiegene“) in die Wirtschaft. Durch ihre Fachkenntnisse und guten Beziehungen zu ihren ehemaligen Ministerien waren sie in der Wirtschaft sehr begehrt. Hier lag natürlich auch eine gewisse Korruptionsgefahr: Eine zweite Karriere war vor allem für diejenigen Beamten aussichtsreich, die während ihrer aktiven Zeit mit den Unternehmen entsprechend kooperiert hatten. ${ }^{101}$

Die amakudari galten als Verbindungsglied zwischen Bürokratie und Wirtschaft und somit als „basic mechanism for Japan’s iron triangle“102. Allerdings gibt es durchaus unterschiedliche Meinungen darüber, wie groß der Einfluss dieser ehemaligen Spitzenbeamten auf das politische System Japans tatsächlich war.

Ab Mitte der 1970er Jahre war die Stellung der Ministerialbürokratie nicht mehr so dominant: „, The bureaucracy is still a major and powerful actor in policymaking, but it now must share the stage with a number of other influential actors. “ ${ }^{103}$ Die bereits genannten zoku giin bildeten zunehmend ein Gegengewicht zur Ministerialbürokratie. Sie waren auf der einen Seite als Vermittler für organisierte Interessen tätig, auf der anderen Seite wurden ihre Beziehungen von den Ministerien genutzt, um Stellen in der Wirtschaft für ausscheidende Beamte zu vermitteln. Die einzelnen Politiker profitierten dabei nicht nur durch Vermittlung von Aufträgen in ihre Wahlkreise, sondern auch von den „Vermittlungshonoraren“, die ein Unternehmen als Spenden an die Wahlkampforganisationen zahlte. „Das Iron Triangle der wechselseitig verflochtenen Beziehungen der Akteure im politischen Prozess stellt Politik in Japan als ein Austauschverhältnis dar, in dem Macht und Einfluß gegen politische Spenden einerseits oder lukrative Ruhestandsposten andererseits zur Wahrung bestimmter Interessen eingesetzt werden. “104

Dieses System trug nicht unwesentlich zum wirtschaftlichen Aufstieg und zur sozialen Stabilität Japans bei. Es war aber andererseits sehr korruptionsanfällig, so dass diese vom „Geld gesteuerte Politik“ (kinken seiji) schon ab den 1970er Jahre als zunehmende Belastung empfunden wurde. ${ }^{105}$

\footnotetext{
${ }^{100}$ Vgl.: Ebd., S. 209.

${ }^{101}$ Vgl.: Kunio, Yoshihara: Sogo Shôsha. The vanguard of the Japanese economy, Oxford, 1982, S. 286.

102 Colignon, Richard; Usui, Chikako: The resilience of Japan's iron triangle, in: Asian Survey, Vol. 41, No. 5/2001, S. 866.

${ }^{103}$ Maramatsu, Michio; Krauss, Ellis S.: A.a.O., S. 540.

104 Blechinger, Verena: Politische Korruption in Japan, a.a.O., S. 217.

${ }^{105}$ Vgl.: Pempel, T.J.: Japan's Creative Conservatism. Continuity under Challenge, in: Castles, Francis G.: The Comparative History of Public Policy, 1989, S. $156 f$.
} 


\subsubsection{Die Staatsstruktur}

Japan ist ein sehr zentralistisch geprägter Staat. Sowohl die Verwaltung, als auch die Wirtschaft und das kulturelle Leben sind auf die Hauptstadt Tôkyô ausgerichtet. Unterhalb der nationalen Ebene gibt es mit den Präfekturen, Großstadtregionen und der Insel Hokkaidô eine regionale Ebene (todôfuken), die 47 Einheiten umfasst. Den lokalen Institutionen ist zwar eine ganze Reihe von Aufgaben übertragen worden, aber es existieren in Japan keine mit Deutschland vergleichbaren föderalistischen Elemente. Vielmehr sind die unteren regionalen und lokalen Ebenen von der nationalen Ebene politisch und finanziell abhängig. ${ }^{106}$ Masumi Junnosuke meint dazu: „[...] It is virtually impossible for a local government to operate without relying on subsidies. “107

Die Gouverneure der Präfekturen verfügen über eine vergleichsweise starke Position. Sie werden getrennt von den Abgeordneten der regionalen Parlamente direkt gewählt. Letztere hatten unter dem 1955er-System nur geringe Einflussmöglichkeiten. Zwar war ein Misstrauensvotum gegen den Gouverneur möglich, „weichere“ Druckmittel wie ein Budgetrecht fehlten hingegen. Erst ab Mitte der 1960er Jahre wuchs die Bedeutung der regionalen und lokalen Ebenen. Mit der bereits genannten Pluralisierung der japanischen Gesellschaft begann das so genannte „Zeitalter des Lokalismus“. Es gab eine ganze Reihe von Bürgerbewegungen mit lokalen Wurzeln. Die Handlungsmöglichkeiten auf der lokalen Ebene sollten daher nicht unterschätzt werden. Vor allem ist die Beteiligung der Bürger auf lokaler Ebene vergleichsweise hoch, wozu auch einige Elemente direkter Demokratie beitragen. ${ }^{108}$ Auf die Details soll hier nicht weiter eingegangen werden, aber: „[...] 'Localism' (jimoto shugi), identification with locality and policy innovation and local interests, is a free-floating resource that can be mobilized for policy innovation and may in fact be necessary for its successful implementation.”109 Dieser Lokalismus bildet gewissermaßen ein Gegengewicht zum relativ zentralistischen japanischen Staat.

Für die Parteipolitik wurde die lokale Ebene zwar erst sehr spät entdeckt, entwickelte sich aber schnell zu einem Rekrutierungsfeld für nationale Politik. Für die Unterhausabgeordneten war die lokale Ebene schon aufgrund des Wahlsystems von großer Bedeutung (s.u.). Hier mussten sie mit ihren lokalen Organisationen Stimmen sammeln, wobei sie auch auf die Unter-

\footnotetext{
${ }^{106}$ Vgl.: Pohl, Manfred: Demokratie auf Japanisch, in: Wehling, Hans Georg: Japan. Kohlhammer Taschenbücher, Bd. 1071, Stuttgart 1985. S. 97ff.; Pempel, T.J.: Prerequisites for Democracy: Political and Social Instititutions, in: Ishida, Takeshi; Krauss, Ellis S. (Hg.): Democracy in Japan, 3.Auflage, University of Pittsburgh Press, Pittsburgh 1994, S. 31.

${ }^{107}$ Masumi, Junnosuke: Contemporary Politics in Japan, a.a.O., S. 263.

${ }^{108}$ Vgl.: Hanai, Hirotada: Seiji-no shikumi, Dayamondo-sha, Tôkyô 1996, S. 194 ff.; Okada, Kenji: A.a.O., S. 173-185; MacDougall, Terry E.: A.a.O., S. 158-166.

${ }^{109}$ MacDougall, Terry E.: Democracy and Local Government in Japan, a.a.O., S. 154.
} 
stützung von Lokalpolitikern oder lokalen Organisationen angewiesen waren, deren Stellung dadurch aufgewertet wurde. ${ }^{110}$

Die japanische Staatsstruktur führte ebenfalls dazu, dass das nationale Parlament als das eigentliche Karriereziel japanischer Politiker galt, wo die Ressourcen verteilt wurden. Attraktive Posten ober- oder unterhalb dieser Ebene waren gar nicht (auf supranationaler Ebene) oder nur begrenzt (auf regionaler Ebene) vorhanden. Die regionale oder lokale Ebene diente lediglich als Einstieg oder Durchgangsstation für den Weg in die nationale Politik. Die Bedeutung der regionalen Parlamente als Rekrutierungsfeld für Unterhausabgeordnete war zwar sehr groß, (finanziell) gleichwertige Auffangpositionen für gescheiterte Unterhausabgeordnete boten sie aber nicht. Ämtersukzessionen wie etwa die Wahrnehmung eines Mandats auf regionaler und nationaler Ebene zur gleichen Zeit waren gesetzlich verboten. Die Rückkehr in die „Provinz“ nach einer verlorenen Unterhauswahl gestaltete sich somit als schwierig und war darüber hinaus wenig attraktiv. Die meisten Akteure unterhalb der nationalen Ebene waren oft nur im Nebenberuf als Politiker tätig. ${ }^{111}$ Der Wechsel zwischen den einzelnen Ebenen war also möglich, wurde meist aber nur in eine Richtung vollzogen. Eine Ausnahme bilden allenfalls die Gouverneursposten in den Präfekturen. ${ }^{112}$

\subsubsection{Rolle und Struktur des Parlaments}

Das japanische Parlament besteht aus zwei Kammern, dem Unterhaus (shûgiin) mit Abgeordneten und dem Oberhaus (eigentlich „Beraterkammer“, sangiin). Wie der Name Beraterkammer schon andeutet, kommt dem japanischen Oberhaus laut Verfassung eine beratende Funktion zu, und es verfügt über wesentlich weniger Einflussmöglichkeiten als das Unterhaus. Zwar muss das Oberhaus Gesetzesvorlagen zustimmen, ein Gesetz kann aber bei Ablehnung durch das Oberhaus und erfolglosen Verhandlungen im Vermittlungsausschuss durch Zweidrittelmehrheit im Unterhaus verabschiedet werden. ${ }^{113}$

Die LDP verfügte während des 1955er-Systems lange Zeit auch im Oberhaus über eine absolute Mehrheit. So konnten die im Unterhaus verabschiedeten Gesetze das Oberhaus problemlos passieren. Dieses „Schattendasein“ des japanischen Oberhauses führte daher zur Forderung nach einer Abschaffung dieser Kammer. Auch die eigentlich vorgesehene Funktion einer Beraterkammer, in der partei- und regionenübergreifend beraten werden sollte, war für viele Kriti-

\footnotetext{
${ }^{110}$ Vgl.: Bruns, Gabriele: A.a.O., S. 76.

${ }^{111}$ Vgl.: Derichs, Claudia; Kerbo Harold: A.a.O., S. 291; Okada, Kenji: A.a.O., S. 181.

112 Vgl.: Pohl, Manfred: Demokratie auf Japanisch, a.a.O., S. 99.

${ }^{113}$ Vgl.: Bruns Gabriele: A.a.O., S. 68.
} 
ker nicht mehr erkennbar. ${ }^{114}$ Spätestens seit 1989, als die LDP ihre Oberhausmehrheit verlor, wurde die „Beraterkammer“ als parteipolitisches Instrument genutzt. ${ }^{115}$

Ein hohes Prestige bot vor allem die Zugehörigkeit zu einem wichtigen Ausschuss des Unterhauses wie etwa dem Haushaltsausschuss. Bis 1993 gab es im Unterhaus etwa 20 Ausschüsse, die in „ständige Ausschüsse“ und „Sonderausschüsse“ unterteilt waren. ${ }^{116}$ Zwar hatte sich der Gesetzgebungsprozess, wie oben beschrieben, in die Ministerien verlagert. Dennoch stellten die Ausschüsse gerade für Oppositionspolitiker eine Möglichkeit dar, Kontakt mit den Verbindungsbüros der Ministerialbürokratie zu halten. Hinzu kam, dass die LDP trotz Parlamentsmehrheit nicht die erforderliche Zahl von Abgeordneten besaß, um in allen Ausschüssen die Mehrheit zu stellen. Hier konnten Oppositionspolitiker mit Boykotten oder Verzögerungstaktiken die LDP zu Zugeständnissen zwingen und für eine gute Presse sorgen.

Wie in Deutschland gab es auch in Japan ab einer bestimmten Anzahl von Abgeordneten einen Fraktionsstatus für eine Partei. Die Zugehörigkeit zu einer Fraktion gab den Abgeordneten neben dem Zugang zu Ressourcen der Fraktion auch Sonderrechte wie etwa das Befragungsrecht. Diese konnten gerade für Oppositionspolitiker sehr werbewirksam sein, denn „die ,Verhöre' durch Abgeordnete, bei dem sich schon mancher Minister blamiert hat, werden im Fernsehen übertragen, und alle Tageszeitungen bringen die Befragungen im Wortlaut. “117

Insgesamt lässt sich sagen, dass die Zugehörigkeit zum Unterhaus einem japanischen Politiker im Vergleich zum Oberhaus mehr Karrierechancen und politischen Einfluss bot. Sämtliche Kabinettsmitglieder mussten laut Verfassung einem der beiden Häuser angehören, zumeist wurden die Kabinettsposten jedoch an Unterhausmitglieder vergeben. ${ }^{118}$ Das Oberhaus gilt dagegen eher als Ausweichposition für verdiente oder ausgeschiedene Unterhausabgeordnete.

\subsubsection{Das Wahlsystem}

\subsubsection{Chûsenkyokusei: Das Wahlsystem mittelgroßer Wahlkreise}

Das japanische Unterhauswahlsystem, das bis 1994 galt, wurde 1947 eingeführt. Es handelte sich um das so genannte chûsenkyokusei, das „Wahlsystem mittelgroßer Wahlkreise“, wie es zeitweise auch in Taiwan und Südkorea angewandt wurde. Die korrekte Bezeichnung ist eigentlich SNTV-MMD (single non-transferable vote - multimember districts). ${ }^{119}$

\footnotetext{
${ }^{114}$ Vgl.: Pohl, Manfred: Demokratie auf Japanisch, a.a.O., S. 88.

${ }^{115}$ Vgl.: Bruns Gabriele: A.a.O., S. 69.

Das 1983 eingeführte Wahlsystem mit 100 Listenplätzen und 152 Direktmandaten unterschied sich von dem des Unterhauses und bildete das Vorbild für die Reform des Wahlsystems 1994.

${ }^{116}$ Vgl.: Yamaguchi, Kazuto: Das parlamentarische System Japans. Wissenschaftliche Dienste des Deutschen Bundestages Nr. 128, Bonn 1998, S. 7.

${ }^{117}$ Pohl, Manfred: Demokratie auf Japanisch, a.a.O., S. 87.

${ }^{118}$ Krauss, Ellis S.: Politics and the Policymaking Process, A.a.O., S. 40.

${ }^{119}$ Vgl.: Klein, Axel: Das Wahlsystem als Reformobjekt. Eine Untersuchung zu Entstehung und
} 
Unter diesem Wahlsystem hatte jeder Wähler eine Stimme, die er einem Kandidaten in seinem Wahlkreis geben konnte. Allerdings erhielten, je nach Wahlkreisgröße, mehrere Kandidaten ein Mandat. Das heißt, in einem 5er-Wahlkreis zogen die fünf Kandidaten mit den meisten Stimmen ins Parlament ein. ${ }^{120} 1992$ wurden die 511 Mandate des Unterhauses in 129 Wahlkreisen vergeben, wobei die durchschnittliche Wahlkreisgröße bei 3,96 lag. ${ }^{121}$

Um eine Mehrheit im Unterhaus zu gewinnen, musste eine Partei also im Schnitt mindestens zwei Kandidaten pro Wahlkreis aufstellen. Für die Kandidaten bedeutete das, dass sie gegen Konkurrenten der eigenen Partei antraten. Da der Wähler nur eine, unübertragbare, Stimme hatte, kämpften mehrere Politiker einer Partei sozusagen um dieselbe Stimme. In Japan wurde für dieses Phänomen das Wort dôshi uchi benutzt, der „Kampf unter Gleichen“. ${ }^{122}$

Die Parteiidentifikation in Japan war nicht allzu sehr ausgeprägt. Die Zahl der Japaner, die in Umfragen angaben, keine Partei zu unterstützen stieg von 25,6\% 1960 auf 35,9\% 1980. Interessanterweise lag diese Zahl höher, je jünger und gebildeter die Befragten waren. ${ }^{123}$

Folglich war die Zahl der Stammwähler relativ gering. Nur 17\% der japanischen Wähler gaben bei jeder Ober- und Unterhauswahl von 1980 bis 1992 einem LDP-Kandidaten ihre Stimme. In ländlichen Gebieten waren es immerhin 25,5\%. Dennoch verfügten die einzelnen Parteien über einen harten Kern von Wählern in bestimmten Schichten oder Regionen. So waren vor allem Bauern und Kleinunternehmer eine „typische“ Wählerbasis für die LDP. Der Wahlkreiszuschnitt begünstigte die eher „traditionell orientierten“ ländlichen Wahlkreise. In den städtischen Ballungszentren lag die erforderliche Stimmenanzahl für einen Mandatsgewinn bis zu dreimal so hoch. Dies stellte einen großen Vorteil für die LDP dar, die ihre Hochburgen vor allem in den ländlich geprägten Gegenden hatte. Ab den 1970er Jahren erreichte die LDP aber auch zunehmend die Bevölkerung der Vorstädte. ${ }^{124}$

Die LDP konnte mit relativ geringer Stimmenzahl eine Mehrheit im Unterhaus erreichen (in den Jahren von 1970 bis 1990 durchschnittlich 36,3\% der abgegebenen Stimmen). ${ }^{125}$ Das hatte, abgesehen vom Wahlkreiszuschnitt, vor allem damit zu tun, dass eine Partei beispielsweise in einem 5er-Wahlkreis besser abschnitt, wenn sie mit relativ wenigen Stimmen den Dritt- bis

\footnotetext{
Auswirkung politischer Erneuerungsversuche am Beispiel Japan. Bonner Japanforschungen Bd. 18, Bier'sche Verlagsanstalt, Bonn 1998, S. 49.

${ }^{120}$ Allerdings gab es eine Mindeststimmen-Anzahl, die ein Kandidat erreichen musste.

${ }^{121}$ Vgl.: Klein, Axel: A.a.O., S. 49-51.

1992 gab es acht 2er, 39 3er, 34 4er, 46 5er und zwei 6er Wahlkreise.

${ }^{122}$ Vgl.: Katô, Shûjirô: Seiji-no shikumi. Nihon-ha kô natte iru, PHP kenkyûsho, Tôkyô 1995, S. 80. (Katô, Shûjirô: Mechanismen der Politik. So ist Japan.)

${ }^{123}$ Vgl.: Masumi, Junnosuke: Contemporary Politics in Japan, a.a.O., S. 386.

${ }^{124}$ Vgl.: Bouisseau, Jean-Marie: Organizing One's Support Base under the SNTV: The Case of Japanese Koenkai, in: Grofman, Bernard et.al. (Hg.): Elections in Japan, Korea and Taiwan under the Single Non-Transferable Vote, The University of Michigan Press 1999, S. 89ff.; Blechinger, Verena: Politische Korruption in Japan, a.a.O., S. 128-129; Bruns, Gabriele: A.a.O., S. 197.

${ }^{125}$ Vgl.: Boisseau, Jean-Marie: A.a.O., S. 89.
} 
Fünftplazierten stellte, als wenn nur einer ihrer Kandidaten eine sehr hohe Stimmenzahl erreichte. In der Regel reichten einem Kandidaten 20 bis 25\% der Stimmen, um sicher ein Mandat zu erringen. ${ }^{126}$

Aber auch wenn es möglich war, mit einer relativ geringen Stimmenanzahl über die hinteren Plätze ins Parlament einzuziehen - 1993 erreichte ein Drittel der Abgeordneten nur 10\% der abgegebenen Stimmen in ihrem Wahlkreis - mussten die Kandidaten bis zum letzten Tag um die Wähler kämpfen, da der Einzug ins Parlament ja vor allem vom Abschneiden der Konkurrenten abhing. ${ }^{127}$ Eine äußerst kleine Veränderung der Stimmenzahl konnte die Wahl entscheiden. Als Beispiel nennt Masumi die Unterhauswahl von 1976, bei der der Stimmenunterschied zwischen dem letztplazierten Gewinner und dem bestplazierten Verlierer in 68 der 130 Wahlkreise weniger als $1 \%$ betrug. In 42 Wahlkreisen entschieden sogar weniger als 0,5\% der Stimmen über den Einzug ins Parlament. Das wirkte sich natürlich auch auf die Sitzverteilung im Unterhaus aus: „Thus, if the LDP had received just 1 percent less or the opposition had gained 1 percent more of the vote, the LDP would have had 20 fewer seats; if the LDP had obtained a mere 1 percent more or the opposition parties 1 percent less, the number of LDP seats would have increased by 22."128

Für die Parteien stellte sich daher das Problem der „richtigen“ Nominierung. Stellte etwa die LDP zu wenig Kandidaten auf, war ihre Mehrheit in Gefahr. Stellte die Partei zu viele Kandidaten auf, verteilten sich die Stimmen der konservativen Wählerschaft auf zu viele Kandidaten. So konnte es z.B. passieren, dass durch eine falsche Strategie (Übernominierung) sowohl der Mandatsträger als auch ein neu aufgestellter Kandidat aus der selben Partei scheiterten. Unter diesem tomodaore („,Gemeinsam untergehen“) litt die SPJ in den 1970er Jahren und ging daher zu der Strategie über, weniger Kandidaten aufzustellen. Auch die anderen Oppositionsparteien stellten oft nur einen Kandidaten pro Wahlkreis auf, so dass während des 1955er-Systems keine Oppositionspartei allein die LDP-Herrschaft gefährden konnte. ${ }^{129}$

Da sich die Größe der potenziellen Wählerschaft möglicherweise bestimmen ließ, nicht aber die Verteilung auf die einzelnen Kandidaten, gab es natürlich auch in der LDP hohe „Fehlerquoten“, die mit der Größe des Wahlkreises anstieg. Auf die Kandidatenbestimmung wird bei

\footnotetext{
${ }^{126}$ Vgl.: Pempel, T.J.: Prerequisites for Democracy: Political and Social Institutions, a.a.O., S. 29.

${ }^{127}$ Vgl.: Iwanaga, Kazuki: Democracy and Electoral Systems in Japan. A Comparative Perspective, Center for Pacific Asia Studies at Stockholm University, Stockholm 1995, S. 16; Blechinger, Verena: Politische Korruption in Japan, a.a.O., S. 131; Boisseau, Jean-Marie: A.a.O., S. 97.

${ }^{128}$ Masumi Junnosuke: Contemporary Politics in Japan, a.a.O., S. 406.

${ }^{129}$ Vgl.: Reed, Steven R.; Bolland, John M.: The Fragmentation Effect of SNTV in Japan, in: Grofman, Bernard et.al. (Hg.): Elections in Japan, Korea and Taiwan under the Single Non-Transferable Vote, The University of Michigan Press 1999, S. 212-218.
} 
den demand-Faktoren noch genauer eingegangen, dennoch lässt sich festhalten, dass bei der Kandidatenaufstellung die innerparteilichen Machtcliquen (habatsu) eine entscheidende Rolle spielten. Da diese aus den Parlamentsabgeordneten bestanden, die in ihrem Wahlkreis Konkurrenz natürlich vermeiden wollten, stellte eher die Unternominierung ein Problem dar. Alles in allem konnte die LDP ihre Fehlerquoten durch ihre Dominanz aber gut verkraften. ${ }^{130}$

Da die Kandidaten größerer Parteien in Konkurrenz mit Kandidaten aus der eigenen Partei standen, war es kaum möglich, einen Wahlkampf auf der Basis politischer Programme zu führen. Ideologische Auseinandersetzungen zwischen Kandidaten der selben Partei hätten schließlich auch dem Image der Partei als „Ganzem“ geschadet und wären wohl von der Parteizentrale auch nicht hingenommen worden. „In order to divide the votes of many party loyalists efficiently, parties must devise an incentive-compatible scheme for grouping party voters with party candidates - a way for candidates to enhance their individual reputations without detracting from the party's overall appeal to voters. "131

Daher wurden wurden seitens der Partei „partikularistische Programme” in den Wahlkreisen unterstützt, für die der jeweilige Kandidat verantwortlich zeichnen konnte und nichts anderes als pork barrel politics darstellten. Der Abgeordnete wurde zum Agenten seines Wahlkreises, der von Tôkyô „Wohltaten“ wie Subventionen oder öffentliche Aufträge in den Wahlkreis lenkte. Dabei hatten LDP-Abgeordneten als Angehörige der Regierungspartei unter den bereits genannten Mechanismen des 1955er-Systems gewisse Vorteile gegenüber Kandidaten der Opposition. $^{132}$

\subsubsection{Kôenkai: persönliche Unterstützerorganisationen der Kandidaten}

Im Wahlkreis selbst waren die Kandidaten auf sich allein gestellt. Gerade die LDP verfügte nicht über die lokale Infrastruktur, einzelne Kandidaten zu unterstützen. Darüber hinaus wäre es für eine lokale Parteiorganisation auch sehr schwer gewesen, den Wahlkampf mehrerer Kandidaten, die gegeneinander antreten, zu organisieren. Dieses Problem hatten alle Parteien, die mehr als einen Kandidaten pro Wahlkreis aufstellten.

Die Abgeordneten waren daher auf den Unterhalt eigener Organisationen in den Wahlkreisen angewiesen, so genannter kôenkai. Seit den frühen 1950er Jahren bauten vor allem konservative Politiker, auch als Folge des sozialen Wandels, diese Organisationen auf: „Land reform, then high-speed growth and mass migration from the countryside, shattered the rural networks

\footnotetext{
${ }^{130}$ Vgl.: Cox, Gary W.; Niou, Emerson: Seat Bonuses under the Single Non-Transferable Vote System: Evidence from Japan and Taiwan, in: Grofman, Bernard et.al. (Hg.): Elections in Japan, Korea and Taiwan under the Single Non-Transferable Vote, The University of Michigan Press 1999, S. 355 ff.; Iwanaga, Kazuki: A.a.O., S. $14 f$.

${ }^{131}$ Cox, Gary W.; McCall Rosenbluth, Frances: The structural determinants of electoral cohesiveness:

England, Japan, and the United States, in: Cowhey, Peter F.; McCubbins, Matthew D.: Structure and Policy in Japan and the U.S., Cambridge University Press, Cambridge 1995, S. 19-80.

${ }^{132}$ Vgl.: Katô, Shûjirô: A.a.O., S. 80; Cox; Rosenbluth: A.a.O., S. 47
} 
of notables. In the megapolis, new channels of political communication had to established to reach millions of uprooted people who had lost their traditional allegiances." 133

Es wird geschätzt, dass in den 1980er Jahren etwa 15 Millionen Wähler, 15 bis 18\% der Wählerschaft, Mitglieder in einer kôenkai waren. Verena Blechinger unterscheidet zwischen so genannten „Spenden-kôenkai“ und „Wahl-kôenkai“ (senkyo-kôenkai). ${ }^{134}$ Während die Erstgenannten dazu dienten, sowohl auf lokaler Ebene als auch auf nationaler Ebene „Mitgliedsbeiträge“ und „Spenden“ von Unternehmen zu sammeln, waren die Wahl-kôenkai die lokale (Wähler-)Basis des Kandidaten.

Herzstück der kôenkai waren zumeist die Sekretäre des Kandidaten. Durchschnittlich 16 feste Mitarbeiter beschäftigte ein Abgeordneter für seine Wahlkreisarbeit. Auch Verwandtschaftsverhältnisse spielten in den kôenkai eine wichtige Rolle. Die Führungspositionen in den kôenkai wurden nicht selten von Verwandten des Abgeordneten besetzt. Unterhalb der Führungsebene gab es bis zu 1.000 so genannte Freiwillige mit bestimmten Funktionen (sewanin). Die Basis bildeten etwa 10.000 registrierte Mitglieder und noch einmal die doppelte Zahl von Sympathisanten (shôkaisha). Zu den sewanin gehörten ebenfalls Freunde und Verwandte des Abgeordneten, die als Multiplikatoren ihrerseits Freunde und Bekannte ansprachen oder örtliche Honoratioren und Unternehmer, die als Meinungsführer einen gewissen Einfluss hatten. ${ }^{135}$ Als Prototyp der kôenkai galt die Unterstützerorganisation des früheren Ministerpräsidenten Tanaka Kakuei, die Etsuzankai, die 313 Ortsgruppen und 95.000 Mitglieder umfasst haben soll. ${ }^{136}$

An dieser Stelle sollte man allerdings hinzufügen, dass oft nur die Hälfte der mit einer kôenkai „verbundenen“ Wähler auch tatsächlich diesen Kandidaten wählte. Boisseau führt dies darauf zurück, dass die Mitgliederzahlen von den Helfern der kôenkai häufig künstlich aufgeblasen wurde. Personen, die angesprochen wurden, den Kandidaten zu unterstützen, wurden sogleich als „Mitglied“ geführt, waren de facto aber keine verlässlichen Wähler. ${ }^{137}$

Eine kôenkai stellte ein Mosaik von Gruppen dar. Häufig waren in die kôenkai eines Unterhausabgeordneten diverse Interessengruppen oder die Unterstützerorganisationen lokaler Politiker eingebunden. Somit waren die persönlichen Unterstützerorganisationen eine Art „Schirmorganisation““138. Darunter befanden sich auch Gruppen, in denen sich die lokalen Unternehmer trafen und Kontakte knüpften, außerdem Frauengruppen (Hausfrauenvereinigungen in der

\footnotetext{
${ }^{133}$ Boisseau, Jean-Marie: A.a.O., S. 99.

${ }^{134}$ Vgl.: Ebd., S. 99f.; Blechinger, Verena: Politische Korruption in Japan, a.a.O., S. 162

${ }^{135}$ Vgl. : Boisseau, Jean-Marie : A.a.O., S. 102-104 ; Katô, Shûjirô: A.a.O., S. 88; Fukui, Haruhiro ; Fukai, Shigeko N. : Pork Barrel Politics, Networks, and local economic Development in Contemporary Japan, in: Asian Survey, Vol. 36, No. 3/1996, S. 282.

${ }^{136}$ Vgl.: Woodall, Brian: Japan under construction: corruption, politics and public work, University of California Press, Berkeley 1996, S. 89.

${ }^{137}$ Vgl.: Boisseau, Jean-Marie: A.a.O., S. 104.

${ }^{138}$ Vgl.: Ebd., S. 89.
} 
Nachbarschaft), Jugendgruppen oder Hobbygruppen. Überhaupt nahmen Freizeitaktivitäten einen großen Teil der kôenkai-Aktivitäten ein. Zwar mussten die kôenkai-Mitglieder aus rechtlichen Gründen Unkostenbeiträge für Getränke oder Ausflüge zahlen, aber diese Beiträge deckten selten die tatsächlichen Ausgaben. ${ }^{139}$

Japan wird häufig als „Geschenkkultur“ bezeichnet. Von den Abgeordneten wurde erwartet, dass sie bei Hochzeiten und Beerdigungen (Geld-)Geschenke gaben. Bei diesen so genannten kankonsôsai (Die Feste zum Erreichen des Erwachsenenalters, Hochzeit und Beerdigung) kamen einige Abgeordneten auf 500 Hochzeiten und 200 Beerdigungen pro Jahr, was Ausgaben von bis zu 30 Mio. Yen bedeutete. ${ }^{140}$

Zwischen dem Kandidaten und den Mitgliedern seiner kôenkai bestand mitunter eine starke persönliche Bindung. Einige Abgeordnete boten über ihre Mitarbeiter sogar Steuerberatungen an. Oft wird angeführt, dass in Japan persönliche Beziehungen und Pragmatismus als cultural features diese Art von Klientelismus verstärken. Loyalitäten, die sich auf Fürsorge und Unterstützung gründen, spielten dabei eine Rolle. Nicht selten werden Vergleiche zwischen den Abgeordneten und den daimyô (Fürsten) der japanischen Feudalzeit gezogen. ${ }^{141}$

Fukui und Fukai erwähnen im Zusammenhang mit den kôenkai die so genannten electoral keiretsu (Linien): „, This is a coalition formed for, and on the basis of mutual benefits between a Diet member and local politicians. “142 Die Bedeutung partikularistischer Politik wurde bereits erwähnt und wird häufig mit dem zentralistischen Staatsaufbau in Zusammenhang gebracht. Während der Parlamentsabgeordnete für die Lokalpolitiker also eine wichtige Verbindung nach Tôkyô darstellte, mobilisierten die örtlichen Politiker z.B. mit Hilfe ihrer Unterstützerorganisationen Wähler für den Abgeordneten. Umgekehrt kam es auch vor, dass Unterhausabgeordnete ihre kôenkai den Lokalpolitikern zur Verfügung stellten. ${ }^{143}$ Beispielsweise nutzte der Sohn des in den Interviews befragten Unterhausabgeordneten Usui die Wahlkampforganisation seines Vaters während des Wahlkampfes für das Regionalparlament 2003.

Ende der 1980er Jahre verfügten die Kandidaten fast aller Parteien über kôenkai. Da die Parteiführung der LDP den Ausbau der kôenkai seit Ende der 1950er Jahre nicht verhindern konnte und wohl auch nicht wollte, überlegte man verschiedene Möglichkeiten, sie in die regionalen Parteistrukturen zu integrieren. Masumi merkt jedoch an: „But were Diet members really going to hand over to the prefectural federations their kôenkai membership rosters, which were the

\footnotetext{
${ }^{139}$ Vgl.: Ramseyer, Mark J.; MacCall Rosenbluth, Frances: Japan’s Political Marketplace, Harvard 1993, S. 24.

${ }^{140}$ Vgl. : Boisseau, Jean-Marie: A.a.O., S. 104-109 ; Katô, Shûjirô: A.a.O., S. 88.

${ }^{141}$ Vgl. : Boisseau, Jean-Marie: A.a.O., S. 117 ; Katô, Shûjirô: A.a.O., S. 88 ; Blechinger, Verena: Politische Korruption in Japan, a.a.O., S. 87.

${ }^{142}$ Fukui, Haruhiro ; Fukai, Shigeko N. : Pork Barrel Politics, Networks, and local economic Development in Contemporary Japan, a.a.O., S. 280.

${ }^{143}$ Vgl. : Ebd., S. 280 ff.
} 
creation of their money and sweat?" ${ }^{144}$ So bildeten die persönlichen Unterstützerorganisationen den Ersatz für die mangelhaften lokalen Parteistrukturen und wurden zum Rückgrat der Wählermobilisierung nicht nur in der LDP.

Grundsätzlich waren nämlich auch die Kandidaten der Opposition den Zwängen japanischer Wahlkämpfe unterworfen. ${ }^{145}$ Das erkannte beispielsweise der LDP-Ministerpräsident Tanaka Kakuei, der diese Tatsache nutzte, um Oppositionspolitiker finanziell zu beeinflussen: „Yet Tanaka understood that, beneath the rhetoric, opposition politicians were still politicians with certain basic exigencies, from providing constituents favors to raising campaign funds. “146 Ausnahmen gab es lediglich bei der KPJ, die über ausgeprägte lokale Strukturen verfügte und bei der Kômeitô, in der die Sekte Sôka Gakkai und ihre Organisationen diese Aufgaben übernahmen.

Der Unterhalt solcher Organisationen war für den einzelnen Abgeordneten sehr teuer. Die kôenkai werden daher in der Literatur oft als „geldfressende Würmer“ und „Kanäle für Wählerbestechung“ kritisiert. ${ }^{147}$ Einige Abgeordnete gaben bis zu 10 Mio. Euro in einem Wahlkampf aus. So wurden die Wahlkämpfe zu so genannten „Service-Schlachten“, in denen die Wähler mit kleinen Geschenken regelrecht gekauft wurden. Der Geldmitteleinsatz in KPJ und Kômeitô hielt sich wegen der lokal gut ausgeprägte Organisationen zumeist in Grenzen. Darüber hinaus mussten sie oft nur einen Kandidaten pro Wahlkreis unterstützt. ${ }^{148}$

Häufige Neuwahlen und die Konkurrenz durch „Parteifreunde“ führten dazu, dass die Kandidaten auch außerhalb der offiziellen Wahlkampfzeit in „Wartestellung“ waren und sozusagen ständig Wahlkampf führten. Dazu trugen ebenfalls die sehr strengen Gesetze zur Wahlkampfführung bei, wie etwa das Verbot von Haustürbesuchen zur Stimmenwerbung für einen bestimmten Kandidaten oder stark reglementierte Werbung in den Medien. Diese Restriktionen erwiesen sich als kontraproduktiv, verlegten die Wahlkampftätigkeiten in die Vorwahlkampfzeit und verstärkten auf diese Weise die Rolle der kôenkai. ${ }^{149}$

\subsubsection{Parteien und Interessenverbände}

\subsubsection{Parteien in Japan}

Auf den ersten Blick ähnelten die japanischen Parteien in ihrer Struktur den europäischen Parteien. Die LDP verfügte 1988 über 5,8 Millionen Mitglieder, doch waren nach Schätzungen nur 50.000 von ihnen wirklich aktiv. Der größte Teil der aktiven Mitglieder bekleidete selbst

\footnotetext{
${ }^{144}$ Masumi, Junnosuke: Contemporary Politics in Japan, a.a.O., S. 237.

${ }^{145}$ Vgl.: Boisseau, Jean-Marie: A.a.O., S. 102 ; 108.

${ }^{146}$ Schlesinger, Jacob M.: A.a.O., S. 127.

${ }^{147}$ Vgl.: Katô, Shûjirô: A.a.O., S. 88 .

${ }^{148}$ Vgl.: Klein, Axel: A.a.O., S. 99-100.

${ }^{149}$ Vgl.: Boisseau, Jean-Marie: A.a.O., S. 94ff.
} 
Posten in der regionalen und lokalen Politik. Viele japanische Parteien wiesen Mängel in ihrer Organisationsstruktur auf, vor allem aber die Liberaldemokratische Partei. Diese verfügte nur über eine sehr schmale Parteibasis und schwach ausgeprägte lokale Strukturen. Diese Aussage trifft allerdings nur zu, wenn man die formale Organisation betrachtet und die kôenkai als informelle Elemente zunächst einmal außer Acht lässt. Der Austausch zwischen lokaler und nationaler Politik war nur sehr schwach ausgeprägt und lief im Wesentlichen über die Abgeordneten des Unterhauses. Die zweimal jährlich stattfindenden Parteitage der LDP, offiziell immerhin das höchste Organ der Partei, waren bis ins letzte Detail vorgeplant und boten selten politische Diskussionen. Auch die meisten lokalen und regionalen Parteiorganisationen existierten nur formal. Tatsächlich stützte sich die LDP in den Wahlkreisen auf lokale Netzwerke wie die kôenkai ihrer Abgeordneten, örtliche Honoratioren oder landwirtschaftliche Kooperativen, die ihre Mitglieder während der Wahlen mobilisierten. ${ }^{150}$

Die SPJ wies zwar größere organisatorische und ideologische Gemeinsamkeiten mit europäischen Parteien auf als die LDP, aber auch sie war auf eine geliehene Basis angewiesen, die im Wesentlichen aus den Gewerkschaften bestand. Allerdings hatte die SPJ unter der Zersplitterung der Gewerkschaften in einen radikaleren und einen gemäßigten Flügel zu leiden, was schließlich zur Abspaltung der DSP führte. Die KPJ verfügte über eine wesentlich besser ausgebildete politische Infrastruktur. Die Kommunisten konnten sich sowohl auf einige Gewerkschaftsverbände stützen als auch auf eine lokal gut ausgeprägte Parteiorganisation.

Auch die Parteiorganisation der Kômeitô war auf lokaler Ebene vergleichsweise gut gefestigt. Allerdings wird häufig darauf verwiesen, dass der Erfolg dieser Partei vor allem auf den Kommunikationsstrukturen der Sôka Gakkai basierte. ${ }^{151}$ Als Beispiel wäre hier die Tageszeitung der Sôka Gakkai, die Seikyô Shinbun, zu nennen, die in den 1970er Jahren eine Auflage von 3 Mio. Exemplaren erreichte. Hrebenar weist darauf hin, dass die Organisation der Kômeitô teilweise etwas undurchsichtig war und auch Kômeitô-Politiker über eigene kôenkai verfügten. Offiziell trennte sich die Partei 1970 von der Sekte. Dennoch blieben die Organisationen eng miteinander verwoben. ${ }^{152}$

Beide Parteien, KPJ und Kômeitô, waren sehr zentralistisch und mitunter autoritär geprägt. Durch ihre ideologische Ausrichtung und relativ gute Organisationsstruktur verfügten die Oppositionsparteien über eine feste und leicht zu mobilisierende Wählerbasis. Ihre Attraktivität auf andere Bevölkerungsgruppen war allerdings sehr beschränkt. ${ }^{153}$ Das zeigen auch die Mit-

\footnotetext{
${ }^{150}$ Vgl.: Rothacher, Albrecht: The Japanese Power Elite, St. Martins Press, New York 1993, S. 27-29.

${ }^{151}$ Vgl.: White, James W.: The Sokagakkai and Mass Society, a.a.O., S. 138.

${ }^{152}$ Vgl.: Hrebenar, Ronald J.: The Komeito Returns: The Party of „Buddhist Democracy“, in: Hrebenar, Ronald J: Japan's new party system, Westview Press, Oxford 2000, S. 183.

${ }^{153}$ Vgl.: Hartmann, Jürgen: Politik und Gesellschaft in Japan, USA und Westeuropa: Ein einführender
} 
gliederzahlen: In den 1980er Jahren waren nur 10\% der Kômeitô-Mitglieder nicht in der Sôka Gakkai. ${ }^{154}$

Innerhalb der LDP gab es bedingt durch die mangelhaft ausgeprägte Parteiorganisation auf den unteren Ebenen relativ wenig Möglichkeiten, mit Hilfe einer typischen Parteikarriere in eine Position auf nationaler Ebene zu gelangen. Einige Oppositionsparteien wie die KPJ boten dagegen durch ihre ausgeprägten Organisationsstrukturen hier wesentlich mehr Möglichkeiten. Kômeitô und SPJ verfügten mit einer Sekte bzw. den Gewerkschaften über typische Rekrutierungsfelder für ihre Abgeordneten (s. 5.1.). Auch gab es in den Parteiapparaten der Oppositionsparteien und deren Vorfeldorganisationen theoretisch mehr Auffangpositionen für Parlamentarier. Zwar war die LDP auf nationaler Ebene sehr eng mit Interessengruppen, besonders aus der Wirtschaft, verbunden, doch diese Verbindungen waren zumeist auf den finanziellen Austausch beschränkt.

\subsubsection{Zoku giin: Vermittler organisierter Interessen}

Eine wichtige Rolle innerhalb der LDP nahm das so genannte Policy Affairs Research Council (PARC - seimu chôsakai) ein. Nach Artikel 40 der LDP-Statuten, mussten sämtliche Gesetzesvorlagen vom PARC genehmigt werden, bevor sie an das Kabinett und später an das Parlament weitergegeben werden konnten. Neben dem Vorsitzenden des PARC, seinem Stellvertreter und einem Exekutivkomitees bildeten 17 ständige Ausschüsse das Herzstück des PARC, die den Aufgabenfeldern der Ministerien entsprachen. ${ }^{155}$

Das System der PARC-Komitees ermöglichte es den LDP-Abgeordneten, sich in bestimmten Politikfeldern zu etablieren: „[...] To distribute to its incumbent candidates effective property rights over aspects of policy making (through cabinet posts and the Policy Affairs Research Council [PARC] committee assignments) thus giving each candidate the opportunity to build his own reputation with voters on policy making." 156 Fördergelder aus Tôkyô für die Infrastruktur wurden in den Wahlkreisen der Vermittlungsarbeit des Abgeordneten innerhalb der Partei-Ausschüsse zugeschrieben. Daher war eine Zugehörigkeit zu den Komitees von fundamentaler Bedeutung für die Abgeordneten. Auf diese Weise blieb auch die Mitgliedschaft in der LDP für den einzelnen Abgeordneten attraktiv. ${ }^{157}$

In der Regel gehörte ein Abgeordneter vier Komitees an, zwei davon entsprachen im Aufgabenbereich seiner Tätigkeit in den Parlamentsausschüssen. Allerdings erforderte die Mitarbeit in einem dieser Komitees inoffiziell, dass der Abgeordnete bereits eine volle Wahlperiode im

\footnotetext{
Vergleich, Campus Verlag, Frankfurt/M. 1983, S. 120-125.

${ }^{154}$ Vgl.: Hrebenar, Ronald J.: The Komeito Returns: The Party of „Buddhist Democracy“, a.a.O., S. 181.

${ }^{155}$ Vgl.: Hanai, Hirotada: A.a.O., S. 93-94; Cox; McCall Rosenbluth: A.a.O, S. 49.

${ }^{156}$ Cox, Gary W.; McCall Rosenbluth, Frances: A.a.O, S. 36.

${ }^{157}$ Vgl.: Ebd., S. 36.
} 
Parlament saß. Innerhalb dieser Komitees fand auch die enge Zusammenarbeit mit der Ministerialbürokratie statt. Abgeordnete, die mindestens drei oder vier Wahlperioden in diesen Komitees tätig waren, bildeten so genannte zoku (policy tribes). ${ }^{158}$

Wie bereits erwähnt, stellten diese Abgeordneten als zoku giin mit ihrem Expertenwissen nicht nur ein Gegengewicht zu der Bürokratie dar, sie waren auch als Vermittler zwischen Bürokratie und Wirtschaft attraktiv. Die von der LDP besetzten Vorsitzenden der Parlamentsauschüsse waren in der Regel solche zoku giin. Für einen Abgeordneten bedeutete die Tätigkeit als zoku giin natürlich eine erhebliche Steigerung seines Einflusses, seiner Kontakte zu Bürokratie und Wirtschaft und damit seiner verfügbaren Ressourcen. ${ }^{159}$ Für die Verteilung von (Regierungs-)Posten waren jedoch die so genannten habatsu von Bedeutung.

\subsubsection{Habatsu: Die innerparteilichen Machtgruppen}

Eines der bedeutendsten Ämter innerhalb der LDP ist das Amt des Parteivorsitzenden und damit des Kandidaten für den Posten des Ministerpräsidenten. Zur Zeit der LDP-Dominanz entsprach die Wahl des Parteivorsitzenden de facto der Wahl des Ministerpräsidenten. Diese Wahl wurde zwar offiziell vom Parteitag vorgenommen, tatsächlich waren es aber die innerparteilichen Machtgruppen, die so genannten habatsu, die die Entscheidungen hinter verschlossenen Türen trafen. ${ }^{160}$

Das Wort habatsu wird oft mit „Faktion“ (abgeleitet vom englischen faction) übersetzt und bezeichnet innerparteiliche Gruppen, die im Bereich der informellen Politik angesiedelt sind. Im Gegensatz zu Richtungen oder Flügeln einer Partei verfügen sie über einen höheren Organisationsgrad. Sie sind auf gemeinsame Ziele verpflichtet, zu denen sowohl inhaltliche Anliegen als auch das bloße Streben nach Macht zählen können. ${ }^{161}$

Als Ursache für die Entstehung von habatsu wird im Fall der LDP zunächst auf die Vereinigung der Liberalen Partei und der Demokratischen Partei verwiesen, deren alten Loyalitätsbeziehungen zumindest die Anfangszeit der LDP überdauerten. Darüber hinaus boten die habatsu „ihren“ Kandidaten im Wahlkampf gegen Konkurrenten der eigenen Partei Unterstützung. Da Kandidaten für den Parteivorsitz von mindestens 30 Abgeordneten nominiert werden mussten, wird auch hier ein Grund für den Aufbau der habatsu gesehen. In der Anfangszeit der LDP gab

\footnotetext{
${ }^{158}$ Vgl.: Cox, Gary W.; McCall Rosenbluth, Frances: A.a.O, S. 50.

${ }^{159}$ Vgl.: Blechinger, Verena: Politische Korruption in Japan, a.a.O., S. 179; Hanai, Hirotada: A.a.O., S. 94.

${ }^{160}$ Vgl. Bruns, Gabriele: A.a.O., S. 84.

${ }^{161}$ Vgl.: Köllner, Patrick: Faktionalismus in politischen Parteien: Der japanische Fall aus konzeptioneller und komparativer Perspektive, in: Seifert, Wolfgang; Weber, Claudia (Hg.): Japan im Vergleich, Iudicum, München 2002, S. 281-288.
} 
es durchaus auch einige ideologische Unterschiede zwischen den habatsu, diese spielten aber eine wesentlich geringere Rolle als bei Richtungskämpfen etwa in der SPJ. ${ }^{162}$

Als weitere Ursache für die Entstehung der habatsu wird die Ausrichtung der japanischen Gesellschaft auf vertikale Gruppen genannt. Diese werden durch so genannte sempai(Ranghöherer) kôhai-(Rangniedrigerer) Beziehungen geprägt, bei denen vor allem Seniorität eine wichtige Rolle spielt und die ein einfaches und stabiles aber auch sehr starres System hervorbringen. ${ }^{163}$

Bemerkenswert an der Struktur vertikaler Gruppen in Japan ist aber, dass die Beziehungen zwischen dem Untergebenen und seinem direkten Vorgesetzten stärker ausgeprägt sind als unter den Angehörigen einer Stufe. Diese Beziehungen werden als „oyabun-(Elternteil) kobun(Kind) Beziehungen“ oder „Patron-Klient-Beziehungen“ bezeichnet. Der oyabun ist weit mehr als nur ein Ranghöherer (sempai), er bietet dem kobun Schutz und Hilfe und kann als Gegenleistung Loyalität und Unterstützung in Machtkämpfen erwarten. ${ }^{164}$

Um in der Gruppenhierarchie aufzusteigen, braucht ein Gruppenmitglied Unterstützung von Rangniedrigeren. Hat er eine große Zahl von eigenen kobun um sich geschart, kann es auch zu einem Bruch mit seinem eigenen oyabun kommen, zu einem Umsturz oder einem Gruppenaustritt. Je stärker also Rangniedrigere geworden sind, desto instabiler wird die Gruppe. Die habatsu in den japanischen Parteien hatten unter ähnlichen Problemen zu leiden. Es kam zu Führungskämpfen und Gruppenaustritten, die sogar zur Bildung einer neuen Partei führen konnten. $^{165}$

Die Struktur der habatsu entsprach im Wesentlichen denen der oben genannten vertikalen Gruppen. Die Patron-Klient-Beziehungen in den habatsu waren zwar sehr kollegial geprägt, dennoch waren die Bindungen des kobun an den oyabun von fundamentaler Bedeutung. Die oyabun, bzw. sempai, nahmen sich der jüngeren Abgeordneten an und unterstützten sie. ${ }^{166}$ Als Gegenleistung brachten die Abgeordneten dem Faktionsführer Loyalität und Dankbarkeit entgegen und waren seine „Schachfiguren“ (shogi-no koma) für parteiinterne Machtkämpfe.

Der Aufstieg innerhalb einer habatsu erfolgte zum einen nach messbaren Kriterien wie der Wiederwahlrate, der Dauer der Faktions-Zugehörigkeit oder den „geleisteten Diensten“, d.h. finanzielle Zuwendungen an einen gemeinsamen „Pool“ zur Unterstützung junger Abgeordne-

\footnotetext{
${ }^{162}$ Vgl.: Ebd., S. 292-297.

${ }^{163}$ Vgl.: Nakane, Chie: Die Stuktur der japanischen Gesellschaft, Suhrkamp, Frankfurt/M. 1992, S. 40-52.

${ }^{164}$ Vgl.: Ebd., S. 64-69.

${ }^{165}$ Vgl.: Ebd., S. 65-80.

${ }^{166}$ Vgl.: Blechinger, Verena: Politische Korruption in Japan, a.a.O., S. 97-98; Köllner, Patrick: Faktionalismus in politischen Parteien, a.a.O., S. 296.
} 
ter. Zum anderen spielten die politische Arbeit und der Einfluss in den Ausschüssen eine wichtige Rolle. Nicht zuletzt waren natürlich auch die persönlichen Beziehungen zu den anderen habatsu-Mitgliedern von Bedeutung. ${ }^{167}$

Der Einfluss einer habatsu richtete sich vor allem nach der Zahl ihrer Mitglieder und den Ressourcen (Spenden, Posten), über die sie verfügte. Erfolgreich unterstützte „Newcomer“, die Aufnahme von unabhängigen Abgeordneten oder sogar das Abwerben von Mitgliedern anderer Gruppen konnten das Kräfteverhältnis innerhalb kürzester Zeit verändern. ${ }^{168}$

Die habatsu waren stark institutionalisiert und verfügten über eine eigene Organisation, die den Aufbau der Zentralpartei widerspiegelten. Das Wirken der habatsu, das sich in der Regel auf die zentrale Ebene der Partei beschränkte, war daher auch relativ transparent. Über jeden LDP-Kandidaten war bekannt, zu welcher habatsu er zählte. ${ }^{169}$

Anfang der 1990er Jahre gab es fünf bis sechs große habatsu innerhalb der LDP, von denen die Takeshita-ha, die Miyazawa-ha und die Mitsuzuka-ha mit bis zu 100 Abgeordneten die „Hauptstrom-Faktionen“ darstellten, und einige so genannte „Nebenstromfaktionen“. Die großen habatsu konnten in der Regel drei bis vier Ministerposten beanspruchen, die kleineren höchstens einen. Je nach Größe wurden die habatsu auch von Interessenverbänden wie dem Keidanren unterstützt. ${ }^{170}$ Der Posten des Ministerpräsidenten war allerdings nicht zwangsläufig der einflussreichste Posten. Einige habatsu-Führer verstanden es, als „graue Eminenzen“ aus dem Hintergrund Partei und Regierung zu steuern. ${ }^{171}$

Auch in der SPJ und der DSP existierten habatsu, die jedoch nie den Institutionalisierungsgrad wie in der LDP erreichten. In der SPJ gab es vor allem ideologische Gründe für die innerparteiliche Fragmentierung. In der zentralistisch geprägten KPJ existierten keine habatsu im eigentlichen Sinn. ${ }^{172}$ Auch in der Kômeitô scheinen Faktionen nur eine untergeordnete Rolle gespielt zu haben. Für Außenstehende waren parteiinterne Richtungskämpfe allerdings sowieso schwer erkennbar. Es sollen aber etwa vier faktionsähnliche Gruppen existiert haben. ${ }^{173}$

Häufig werden die habatsu als feudalistisch-patriarchalische Strukturen in der japanischen Politik betrachtet. Für die Parteien konnten die habatsu aber durchaus eine positive Rolle spie-

\footnotetext{
${ }^{167}$ Vgl.: Rothacher, Albrecht: A.a.O., S. 17.

${ }^{168}$ Vgl.: Rothacher, Albrecht: A.a.O., S. 21.

${ }^{169}$ Vgl.: Köllner, Patrick: Faktionalismus in politischen Parteien, a.a.O., S. 295-297.

${ }^{170}$ Vgl.: Rothacher, Albrecht: A.a.O., S. 21.

${ }^{171}$ Vgl.: Blechinger Verena: Politische Korruption in Japan, a.a.O., S. 99.

${ }^{172}$ Vgl.: Köllner, Patrick: Faktionalismus in politischen Parteien, a.a.O., S. 290.

${ }^{173}$ Vgl.: Hrebenar, Ronald J.: The Komeito Returns: The Party of „Buddhist Democracy“, a.a.O., S. 185.

Eine Auseinandersetzung gab es dagegen zwischen Partei und Sekte, als die Sôka Gakkai eine Kooperation mit der KPJ ankündigte, um gemeinsam für den Weltfrieden zu kämpfen, während die Kômeito zur gleichen Zeit einen antikommunistischen Kurs einschlug.
} 
len, etwa als Transmissionsriemen für Aushandlungsprozesse. ${ }^{174}$ Je nach Partei übernahmen die habatsu auch die Funktion, Minderheiteninteressen in den politischen Prozess einzubringen. Klassische Funktion blieb jedoch die Verteilung von Macht und Posten innerhalb der Partei. Schattenseiten der habatsu waren die Korruptionsanfälligkeit und die Machtkämpfe, die das Image der LDP schwer beschädigten.

Für die Abgeordneten war die Funktion der habatsu recht klar. Wer sich einer starken habatsu angeschlossen hatten, erhielt dort Wahlkampfmittel und wurde mit Posten in Partei und Regierung versorgt. ${ }^{175}$ Somit war die Zugehörigkeit zu einer Faktion und die Bindung an einen einflussreichen oyabun für die Karriere(planung) eines japanischen (LDP-) Politikers von größter Bedeutung.

\subsubsection{Die Politikfinanzierung}

Die Politikfinanzierung in Japan bot unter dem 1955er-System ständig Anlass zur Kritik. Eine intransparente Spendenpraxis, hohe Wahlkampfkosten und Skandale wie die LockheedAffäre führten dazu, dass die japanische Politik als äußerst korrupt galt.

Die Einnahmenstrukturen der einzelnen Parteien unterschieden sich erheblich. Die LDP war vor allem auf Spenden aus der Wirtschaft und von verschiedenen Interessenverbänden angewiesen. So gingen 1984 90\% der Spenden aus der Wirtschaft, etwa 4,5 Milliarden Yen, an die Liberaldemokraten. Während SPJ und DSP hauptsächlich von den Gewerkschaften unterstützt wurden, finanzierten sich KPJ und Kômeitô auf zentraler Ebene vor allem durch ihre Unternehmungen, etwa im Fall der KPJ durch die Parteizeitung Akahata (Rote Fahne). ${ }^{176}$

Um die Spenden an die LDP zu bündeln, wurde die kokumin kyôkai (Staatsbürgervereinigung, ab 1975 kokumin seiji kyôkai) gegründet. Ein großer Teil der Spenden ging jedoch direkt an die einzelnen Faktionen. Masumi weist darauf hin, dass zwischen 1955 und 1962 etwa 47\% der Spenden über die Zentralpartei an die habatsu gingen. Das wirkte sich auch auf die innerparteilichen Machtverhältnisse aus: „Thus, a large amount of funds flowed into the factions from sources other than party headquarters. The amount of funds that did not appear in the official record - probably primarily factional or individual funds - cannot be determined. [...] In fact, the factions' prosperity may have been correlated negatively with the flow of funds to party headquarters." 177

\footnotetext{
${ }^{174}$ Vgl.: Köllner, Patrick: Faktionalismus in politischen Parteien, a.a.O., S. 287-300; Nakane, Chie: A.a.O., S. 81. ${ }^{175}$ Vgl.: Kasawara, Morio: Asahi kiwado. Bessatsu seiji, Tokyo 1997, S. 62.

${ }^{176}$ Vgl.: Klein, Axel: A.a.O., S. 98-100; Blechinger: Auf dem Weg zu „sauberer Politik“ und transparenten Strukturen? in: Borchert, Jens; Leitner, Sigrid; Stolz, Klaus (Red.): Politische Korruption. Jahrbuch für Europa- und Nordamerika-Studien 3, Leske+Budrich, Opladen 2000, S. 155-156.

${ }^{177}$ Masumi, Junnosuke: Contemporary politics in Japan, a.a.O., S. 224.
} 
Durch den Unterhalt der kôenkai und die Wahlkampfführung waren Wahlkämpfe für die einzelnen Politiker äußerst kostenintensiv. Die Ausgaben eines Abgeordneten lagen im Schnitt bei 100 Mio. Yen jährlich. In Wahljahren stiegen die Ausgaben bis auf das Dreifache an. Bis 1994 existierte eine Ausgabengrenze für Wahlkampfaktivitäten von 15 bis 20 Mio. Yen, an die sich die meisten Politiker aus Angst vor Sanktionen hielten. Dafür wurden aber die Aktivitäten unmittelbar vor den Wahlkämpfen ausgeweitet. ${ }^{178}$

Da es im 1955er-System keine öffentliche Parteienfinanzierung gab, standen die Politiker vor dem Problem, ständig Geld besorgen zu müssen, um die immensen Kosten ihrer Wahlkreisaktivitäten zu decken. Nach einer Studie von 1987 machten Abgeordnetenbezüge 14,9\% der Einnahmen aus, Zuwendungen von Partei und habatsu 8,2\%. Hinzu kamen Einnahmen aus Aktiengewinnen, Immobilienspekulationen oder Krediten. Den größten Posten nahmen die Erlöse aus Fund Raising Partys (16\%) und politischen Spenden (43\%) ein. ${ }^{179}$

Regelungen zu politischen Spenden waren im „Gesetz zur Regelung politischer Gelder“ (seiji shikin kiseihô) festgelegt, das allerdings erhebliche Lücken aufwies. Spendenobergrenzen wurden zumeist durch den Aufbau mehrerer (Spenden-)kôenkai umgangen. So entwickelten sich regelrechte Spendennetzwerke einzelner Politiker mit bis zu 30 Organisationen! Ein weiteres Schlupfloch bot der Verkauf von Eintrittskarten für Fund-Raising-Partys oder Unternehmensspenden, die zerstückelt wie monatliche Gehälter überwiesen wurden. ${ }^{180}$

Amtsinhaber hatten es gegenüber ihren Herausforderern einfacher, ihre Wahlkämpfe zu finanzieren, wenn diese nicht gerade über ein großes Vermögen verfügten. Zum einen wurden Abgeordnete bei Bedarf von ihren habatsu unterstützt, zum anderen waren sie für Unternehmensspenden attraktiver. Das galt gerade dann, wenn sie als zoku giin über einen gewissen Einfluss innerhalb der Ausschüsse verfügten. Allerdings entsprachen die Beziehungen zwischen organisierten Spendern und Politikern oft regelrechten „Dienstleistungsverträgen“. Viele japanische Politiker agierten daher in den Grauzonen der Parteienfinanzierung. ${ }^{181}$

\footnotetext{
${ }^{178}$ Blechinger, Verena: Auf dem Weg zu ,sauberer Politik“ und transparenten Strukturen? Korruption und Selbstreinigung in der japanischen Politik, a.a.O., S. 126-153.

${ }^{179}$ Vgl.: Ebd., S. 153-156.

${ }^{180}$ Vgl.: Blechinger: Politische Korruption in Japan, a.a.O., S. 225-233.

${ }^{181}$ Vgl.: Blechinger: Auf dem Weg zu „,sauberer Politik“ und transparenten Strukturen? Korruption und Selbstreinigung in der japanischen Politik, a.a.O., S. 156-157.; Rothacher, Albrecht: A.a.O., S. 112-113.
} 


\subsection{Zusammenfassung: Die Opportunitätsstruktur des 1955er-Systems}

Die Auswirkungen der Opportunitätsstruktur auf die Abgeordneten und deren Karriereverläufe lässt sich wie folgt zusammenfassen.

- Die Opportunitätsstruktur brachte vor allem in der LDP einen Politikertypus hervor, der dem des „politischen Unternehmers“ sehr nahe kommt. Die Bedingungen des Wahlsystems, die zentralistische Staatsstruktur und die mangelnden lokalen Strukturen der Parteien förderten die Personalisierung der Wahlkämpfe und einen durch traditionelle Elemente verstärkten Klientelismus. Die Rolle des Abgeordneten wird daher oft mit dem eines „Patrons in einem klientelistischen System“ ${ }^{182}$ verglichen.

- Eine besondere Rolle spielten die persönlichen Unterstützerorganisationen der Kandidaten: „The kôenkai may be said to take the place of the local party organization or the temporarily mobilized volunteer organization of American elections but to bear some resemblance to the vote mobilization to the old-fashioned political machine." 183

- Auf der zentralen Ebene waren die Abgeordneten fest in die Machtstrukturen der Parteien eingebunden. Innerhalb der LDP waren Karrierewege und Aufstiegsmechanismen durch die habatsu und im Parlament durch die Zugehörigkeit zu bestimmten zoku stark vorgegeben. Von der LDP sprach man als „faction- and kôenkai-based mass party“184.

- Der hohe Finanzbedarf japanischer Politiker und eine fehlende staatliche Politikfinanzierung machten die Abgeordneten abhängig von Interessenverbänden und der Wirtschaft. In der LDP führte dies zur Entstehung des Iron Triangle, in dem der Politiker Vermittler zwischen organisierten Interessen und Bürokratie war.

- Auffangpositionen außerhalb des Parlaments waren nur in sehr begrenztem Umfang vorhanden. Die mangelnden Parteistrukturen boten kaum Möglichkeiten nach einer verlorenen Wahl in Parteiämter zu wechseln. Fehlende Möglichkeiten einer Ämtersukzession behinderten, von einigen Ausnahmen abgesehen, die Rückkehr auf die regionale Ebene. Auffangpositionen auf supranationalen Ebenen standen ebenfalls nicht zur Verfügung und führten zu einer relativ geringen „sozialen Mobilität“.

Diese Bedingungen brachten gewisse Karrieremuster hervor. Sie lassen sich an den sozialen Hintergründen japanischer Unterhausabgeordneter ablesen wie beispielsweise typischen Rekrutierungsfeldern, Bildungs- und Altersstrukturen.

\footnotetext{
${ }^{182}$ Blechinger, Verena: Politik und Familienbande, a.a.O., S. 78.

${ }^{183}$ Krauss, Ellis S.: Politics and the Policymaking Process, a.a.O., S. 44.

${ }^{184}$ Masumi, Junnosuke: Contemporary Politics in Japan, a.a.O., S. 239.
} 


\subsection{Der Rekrutierungspfad des Erbabgeordneten}

\subsubsection{Zur sozialen Herkunft japanischer Abgeordneter}

Betrachtet man die soziale Herkunft japanischer Abgeordneter, die Tätigkeiten, die sie vor ihrer Wahl ins japanische Unterhaus ausübten, kann man bestimmte prä-professionelle Rekrutierungsfelder und Karrieremuster herausarbeiten. Diese unterschieden sich erwartungsgemäß je nach Partei. Mit Fokus auf LDP und SPJ, als die beiden größten Parteien während des 1955erSystems, kann man vor allem vier typische Berufsfelder nennen, aus denen sich die Abgeordneten rekrutierten. Als Beispiel sollen hier die Abgeordneten des Unterhauses von 1990 dienen. $^{185}$

\subsubsection{Berufsgruppen}

Die größte Gruppe innerhalb der japanischen Parlamentsabgeordneten auf nationaler Ebene bildeten ehemalige Lokal- und Regionalpolitiker. Etwa 25\% der japanischen Unterhausabgeordneten 1990 waren aus den regionalen Parlamenten oder von einem Gouverneursposten in das Unterhaus gewechselt. Sie zählten damit zu den typischen Laufbahnpolitikern, die sich aus der Lokal- über die Regionalpolitik hochgearbeitet hatten. Der Anteil dieser Abgeordneten war in allen Parteien etwa gleich groß. 66 der 275 LDP-Abgeordneten waren vor ihrem Einstieg in die nationale Politik auf regionaler Ebene tätig gewesen. Diese Regionalpolitiker verfügten in der Regel bereits über eigene Wahlkampforganisationen und lokale Netzwerke und hatten daher gute Chancen, ins Parlament gewählt zu werden. Der Anteil der Abgeordneten, die direkt aus der Lokalpolitik in das Unterhaus gewählt wurden, war dagegen vergleichsweise gering. ${ }^{186}$

Erfahrungen in der lokalen und regionalen Politik besaßen für die Politiker aller Parteien eine große Bedeutung. Es darf auch nicht vergessen werden, dass gerade Oppositionparteien wie die kômeitô zunächst auf lokaler Ebene in den urbanen Gebieten erfolgreich waren: „Effective participation in local office also tended to what the opposition's appetite for national power and generally contributed to its development of moderate and realistic topics. “187

Ein anderes typisches Rekrutierungsfeld für die LDP waren die Beamten der Ministerialbürokratie. Sie stellten 1990 knapp ein Viertel der LDP-Abgeordneten im Unterhaus. Grund für die hohe Zahl von ehemaligen Ministerialbeamten war das frühe Pensionsalter japanischer Beamter. Ab einer bestimmten Karrierestufe konnte nur noch ein kleiner Teil der Ministerialbeamten befördert werden. Wer bei der Auswahl nicht berücksichtigt wurde, beendete in der Re-

\footnotetext{
${ }^{185}$ S. Anhang.

${ }^{186}$ Vgl.: Yomiuri Shinbun: Gekihen-no seijisentaku. 89san 90shûgiinsen tettei bunseki, Yomiurishinbun-sha, Tôkyô 1990, S. 202 ff. (Yomiuri Shinbun: PolitischeAuswahl im Umbruch. Analyse der Wahlen 1989 zum Oberhaus und 1990 zum Unterhaus.); Derichs, Claudia; Kerbo, Harold: A.a.O., S. 293.

${ }^{187}$ McDougall, Terry E.: A.a.O., S. 160.
} 
gel mit etwa 50 bis 55 Jahren seine Karriere im Ministerium und ging in den Ruhestand. Um aber die Zeit bis zum Erhalt der Pension von 65 Jahren zu überbrücken, begannen viele von ihnen eine zweite Karriere in der Wirtschaft oder in der Politik. ${ }^{188}$

Zwar gab es eine Frist von zwei Jahren, während der Beamte nicht in den Unternehmen arbeiten durften, mit denen sie während ihrer Dienstzeit zu tun hatten. Sie wurden aber in der Regel von staatlicher Seite in lukrative Positionen vermittelt. Von 100 börsennotierten Firmen beschäftigten im Jahr 199167 Unternehmen ehemalige Spitzenbeamte. Vor allem in der Baubranche und in der Stahlindustrie fanden viele Beamte einen zweiten Job. ${ }^{189}$

Für ehemalige Ministerialbeamte, die in die Privatwirtschaft wechseln, wird häufig der Begriff amakudari („Vom Himmel Herabgestiegener“) benutzt. Chalmers Johnson hingegen wendet diese Begriff auch auf Beamte an, die nach ihrer Pensionierung in öffentliche Firmen (public companies) oder in die Politik gehen. ${ }^{190}$ Colignon und Usui ordnen diesen beiden Karrierepfaden jedoch eigene Bezeichnungen zu:

\section{Abbildung 7: Karrierepfade ehemaliger Ministerialbeamter}

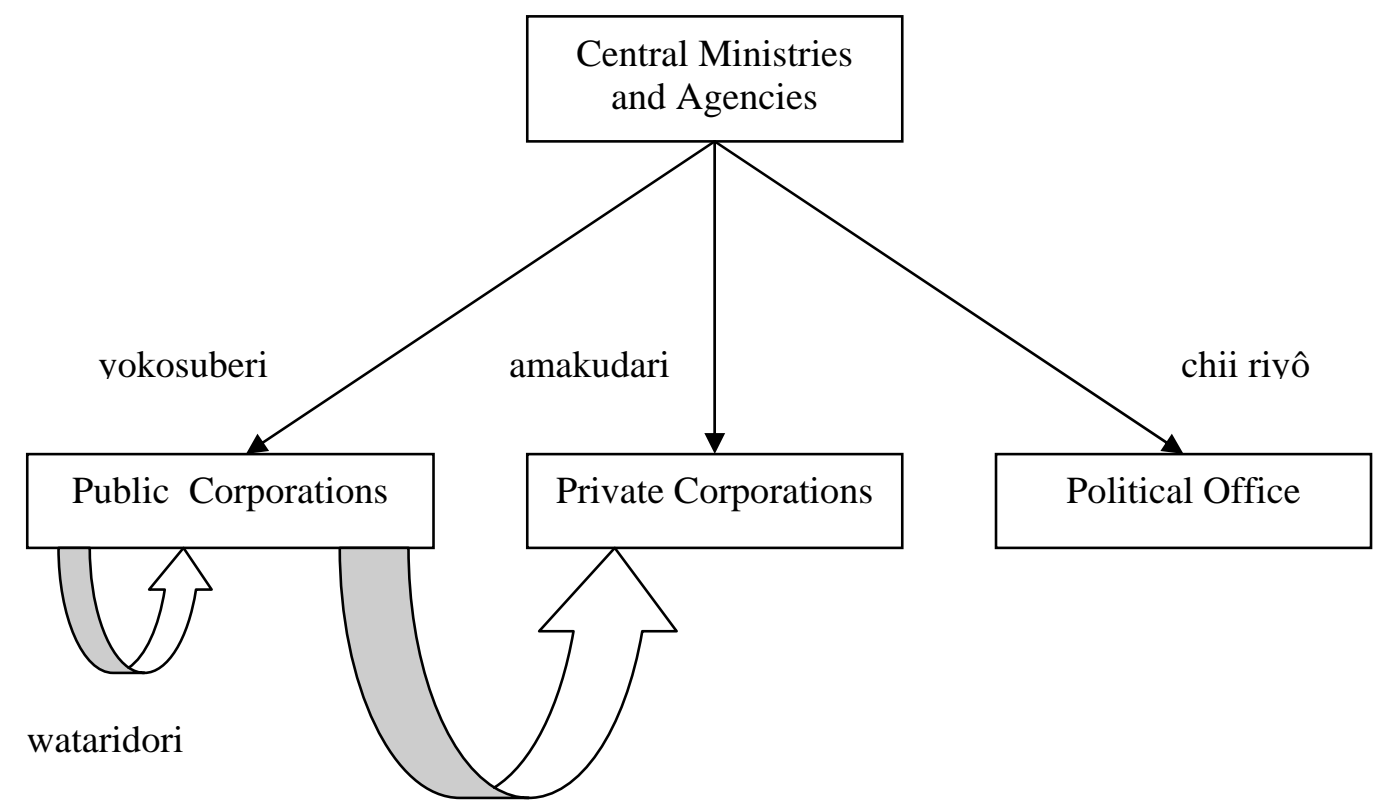

Nach: Colignon, Richard; Usui, Chikako: The resilience of Japan’s iron triangle, in: Asian Survey, Vol. 41, No. 5/2001, S.

\footnotetext{
${ }^{188}$ Vgl.: Blechinger, Verena: Politische Korruption in Japan, a.a.O., S. 202.

${ }^{189}$ Vgl.: Schaede, Ulrike: The “Old Boy” Network and Government-Business Relationships in Japan, in: Journal of Japanese Studies, Vol. 21, No. 2/1995, S. 296-307.

${ }^{190}$ Vgl.: Colignon, Richard; Usui, Chikako: A.a.O., S. 868.
} 
Gemäß diesem Schema gibt es Spitzenbeamte, die als chii riyô („die Position nutzen“) in die Politik gehen, als amakudari in private Unternehmen oder als yokosuberi (,zur Seite ausweichen“) in ein öffentliches Unternehmen. Dabei kann es durchaus Wechsel innerhalb beziehungsweise zwischen den einzelnen Bereichen geben (wataridori).

Der gute Kontakte zu Spitzenpolitikern der langjährigen Regierungspartei LDP und den zoku giin, von denen viele selbst einmal hohe Beamte gewesen waren, erleichterte vielen ehemaligen Beamten eine Kandidatur und eine Karriere in der Politik. Außerdem konnten sie Kontakte zu ihren ehemaligen Ministerien nutzen, um sich selbst als zoku giin zu profilieren. Diese Netzwerke von ehemaligen und noch aktiven Ministerialbeamten ermöglichten einen schnellen Aufstieg innerhalb der LDP-Fraktion. In den japanischen Regierungen waren, wie bereits erwähnt, viele ehemalige Bürokraten. Die engen personellen Verknüpfungen zwischen Ministerialbürokratie und LDP werden auch an der Tatsache deutlich, dass über 90\% der ehemaligen Beamten im Unterhaus für die LDP ihr Mandat ausübten. ${ }^{191}$

Die dritte große Berufsgruppe, vor allem innerhalb der LDP, waren die persönlichen Sekretäre von Abgeordneten (ebenfalls etwa 25\% der LDP-Abgeordneten). Sie verfügten zum einen über Erfahrungen mit der Wahlkreisarbeit und der Führung der Wahlkreisorganisationen, zum anderen über gute Beziehungen in die nationale Politik. Bei einem Rücktritt ihres Abgeordneten hatten die Sekretäre oft die Chance, dessen Wahlkreis zu übernehmen.

Ein gutes Beispiel ist der ehemalige Ministerpräsident Kaifu Toshiki (1989-1991). Kaifu wurde nach seinem Universitätsabschluss Sekretär eines Abgeordneten, dessen Wahlkreis er 1960 im Alter von nur 29 Jahren übernahm. So gelangte er recht früh in verschiedene hohe Regierungsämter und war für japanische Verhältnisse jung, als er mit 58 Jahren japanischer Ministerpräsident wurde. ${ }^{192}$

Ein weiteres wichtiges Rekrutierungsfeld vor allem für die Oppositionsparteien waren die mit den Parteien verbundenen Interessengruppen wie etwa Gewerkschaften. 41 der 136 SPJAbgeordneten waren ehemalige Gewerkschaftsfunktionäre. Wie in anderen westlichen Demokratien waren die Sozialisten und Kommunisten eng mit Gewerkschaftsverbänden verknüpft. In einigen Fällen bestand eine große personelle Abhängigkeit der Parteien von den Gewerkschaften. Allerdings wurden auf diese Weise auch die Richtungskämpfe der Gewerkschaften in die Partei weiter getragen.

In der buddhistischen Partei Kômeitô gab es viele Abgeordnete, die aus mit der Partei verbundenen Gruppen, zumeist Einrichtungen der Sekte Sôka Gakkai, stammten. Auch der Anteil

\footnotetext{
${ }^{191}$ Vgl.: Yomiruri Shinbun: Gekihen-no seijisentaku, a.a.O., S. 202ff.; Blechinger, Verena: Politische Korruption in Japan, a.a.O., S. 202.

${ }^{192}$ Vgl.: Kagaya, Katsuo: A.a.O., S. 182.
} 
der Parlamentarier, die vorher in der Partei selbst beschäftigt waren, lag vergleichsweise hoch. Etwa ein Drittel der Kômeitô-Abgeordneten rekrutierte sich aus diesen beiden Berufsfeldern. ${ }^{193}$ Neben den genannten Parteien gab es 1990 noch eine Reihe kleinerer politischer Gruppierungen im Parlament. 21 der 512 Abgeordneten waren parteilos. Unter ihnen gab es relativ viele Politiker aus der Regionalpolitik und einige Beamte. ${ }^{194}$

Insgesamt 70\% der japanischen Unterhausabgeordneten rekrutierten sich aus diesen vier Gruppen. Selbstverständlich verliefen nicht alle Karrieren so „linear“. Untersuchungen beziehen häufig nur die zuletzt ausgeübte Tätigkeit ein. Viele ehemalige Sekretäre von Abgeordneten gingen beispielsweise zunächst in die Regionalpolitik, wo sie politische Erfahrungen sammelten und eine Basis aufbauten, bevor sie für das Unterhaus kandidierten. Dennoch lässt sich sagen, dass es zumindest bei den Beamten und den Regionalpolitikern kaum Überschneidungen gab: „High-level bureaucrats expand their spheres of influence by scattering subsidies from the top of the lobbying network and pork barrel apparatus, using these spheres as a base of electoral support to become Diet members. Locally elected officials nurture their electoral base and become Diet members by scrambling for subsidies at the base of the pork barrel apparatus. "195

Wegen der schlecht ausgeprägten lokalen Organisationsstrukturen spielten die Parteiorganisationen zumindest in LDP und SPJ als typisches Rekrutierungsfeld nur eine untergeordnete Rolle. Von 411 Abgeordneten, die für diese beiden Parteien 1990 im Unterhaus saßen, waren nur acht Abgeordnete von Parteiposten ins Parlament gewechselt.

Die restlichen Abgeordneten verteilten sich auf verschiedene Berufe, von denen Anwälte (28 Abgeordnete) die größte Gruppe stellten. Innerhalb der LDP rekrutierten sich verhältnismäßig viele Abgeordnete aus Selbstständigen und Angestellten. ${ }^{196}$ Allerdings muss hinzugefügt werden, dass der Anteil der Rechtsanwälte im japanischen Unterhaus im Unterschied zu anderen westlichen Demokratien doch relativ gering war. ${ }^{197}$

Für die Zeit des 1955er-Systems lassen sich im japanischen Unterhaus also je nach Partei bestimmte prä-professionelle Berufs- und Rekrutierungsfelder identifizieren. Innerhalb der LDP gab es sogar je nach Faktion gewisse Unterschiede. In einigen habatsu waren bestimmte Be-

\footnotetext{
${ }^{193}$ Vgl.: Yomiruri Shinbun: Gekihen-no seijisentaku, a.a.O., S. $202 f f$.

${ }^{194}$ In einigen Fällen befanden sich unter den Abgeordneten auch so genannte tarento („Talente“). Dabei handelte es sich um Prominente aus Medien, Sport oder Kultur, die aufgrund ihrer Popularität gewählt wurden und danach oft in die Fraktionen der Parteien aufgenommen wurden.

Vgl.: Kerbo, Harold R.: Who rules Japan? The inner circle of economic and political power, Praeger Publishers, London 1995, S. 105; Wada, Hideki: Nagatachô “seishinbunseki” hôkoku, Shôgakukanbunko, Tôkyô 2002, S. 12. (Wada, Hideki: Eine psychologische Analyse von Nagatachô.)

195 Masumi, Junnosuke: Contemporary Politics in Japan, a.a.O., S. 205.

${ }^{196}$ Vgl.: Yomiruri Shinbun: Gekihen-no seijisentaku, a.a.O., S. $202 \mathrm{ff}$.

${ }^{197}$ Vgl.: Krauss, Ellis S.: Politics and Policymaking, a.a.O., S. 41.
} 
rufsgruppen stärker vertreten als in anderen. So erreichten die ehemaligen Ministerialbeamten in einigen Gruppen einen Anteil von über 50\%. ${ }^{198}$

\subsubsection{Alter, Bildung, Geschlecht}

Betrachtet man die Biographien japanischer Unterhausabgeordneter, gibt es weitere übereinstimmende Charakteristika etwa bei Bildung und Alter. Ein Blick auf den Bildungshintergrund japanischer Abgeordneter zeigt, dass die politische Klasse Japans über eine recht homogene Bildungsstruktur verfügte. Nicht nur der größte Teil der Ministerialbeamten, auch die Mehrheit der Abgeordneten hatte an den Eliteuniversitäten Japans studiert, zu denen vor allem die staatlichen Universitäten Tôkyô (Tôdai) und Kyôto sowie die privaten Universitäten Waseda und Keiô gehören. ${ }^{199}$ Fast die Hälfte der Unterhausabgeordneten hatte an einer der großen Eliteuniversitäten Japans studiert. Besonders häufig sind ehemalige Studenten der Universität von Tôkyô unter den Parlamentariern anzutreffen ${ }^{200}$ Bis heute sind die Absolventen dieser Hochschulen geradezu prädestiniert für eine Karriere in Politik und Wirtschaft. Schnell bildeten sich Netzwerke von Studienkollegen, so genannte gakubatsu, heraus: „So kann sich z.B. ein junger Politiker, der an der Tôdai studiert hat, bei Problemen zu Beginn seiner Karriere an eine Reihe von sempai innerhalb der Partei wenden und aufgrund der gemeinsamen Zugehörigkeit zur Gruppe der ehemaligen Tôdai-Studenten mit Rat und Hilfe rechnen. “201

Unter den Ministerpräsidenten Japans war der Anteil von Absolventen dieser Eliteuniversitäten dementsprechend hoch. Nicht zuletzt deshalb, weil ein Großteil von ihnen aus der Ministerialbürokratie stammte, die sich ja vorwiegend aus der Tôdai rekrutierte. Eine berühmte Ausnahme bildete lediglich Ministerpräsident Tanaka Kakuei, der trotz seiner einfachen Herkunft zu einem der mächtigsten Politiker der japanischen Nachkriegszeit aufstieg. ${ }^{202}$

Ein weiteres Kennzeichen des japanischen Abgeordneten ist das relativ hohe Durchschnittsalter. Viele japanische Abgeordnete kamen erst spät in die Politik, wenn sie bereits eine andere berufliche Laufbahn hinter sich hatten. Als Beispiel können hier die Ministerialbeamten dienen, die während ihrer aktiven Laufbahn nicht für politische Ämter kandidieren durften. Das bedeutete, dass sie erst nach ihrer Pensionierung in die Politik wechseln konnten, wenn sie ihre Beamten-Karriere nicht früher beenden wollten. Dann allerdings hätten sie keine Möglichkeit mehr gehabt, bei einer Wahlniederlage in ihren alten Beruf zurückzukehren. ${ }^{203}$

\footnotetext{
${ }^{198}$ Vgl.: Masumi, Junnosuke: Contemporary Politics in Japan, a.a.O., S. 215.

${ }^{199}$ Vgl.: Koh, Byung Chol: Japan’s Administrative Elite, University of California Press, Berkeley 1989, S. 86ff.; Ishida, Hiroshi: Social Mobility in Contemporary Japan, Macmillan Press, Basingstoke 1993, S. 154.

${ }^{200}$ S. Anhang.

${ }^{201}$ Blechinger, Verena: Politische Korruption in Japan, a.a.O., S. 120.

${ }^{202}$ Vgl.: Kerbo, Harold R.: A.a.O., S. 102.

${ }^{203}$ Vgl.: Klein, Axel: A.a.O., S. 50; Derichs, Claudia; Kerbo, Harold: A.a.O., S. 295.
} 
Das hohe Einstiegsalter zeigt sich bei einer Analyse der Abgeordneten, die 1990 neu ins Unterhaus einzogen. Von den 1990 neu ins Parlament gewählten 133 Abgeordneten waren 47 bereits über 50 Jahre alt. 18 Abgeordnete waren sogar über 60 Jahre alt. Die bereits beschriebenen Aufstiegsmechanismen innerhalb der LDP-Fraktion, die sich unter anderem nach Seniorität bzw. Dauer der Parlamentszugehörigkeit richteten, führten daher zu einem hohen Durchschnittsalter unter japanischen Spitzenpolitikern. Wechselte beispielsweise ein ehemaliger Ministerialbeamter mit 55 Jahren ins Parlament, so war er nach den für eine Spitzenposition erforderlichen vier bis fünf Wiederwahlen fast 70 Jahre alt. ${ }^{204}$ Stockwin nennt sogar sieben Wiederwahlraten als „Wendepunkt“ für japanische Abgeordnete. Oberhalb dieser Schwelle erhielt fast jeder LDP-Abgeordnete einen Posten in der Regierung. ${ }^{205}$

Die Rolle der Frauen in der japanischen Politik ist dagegen als gering einzuschätzen. Zwar gab es einige berühmte Ausnahmen wie die ehemalige SPJ-Vorsitzende Doi Takako: „Es war die Vorsitzende der oppositionellen Sozialdemokratischen Partei, die Ende der 1980er Jahre für den sogenannten ,Madonna-Boom' in Japan sorgte und Hoffnungen auf eine stärkere Beteiligung von Frauen in der Politik weckte. Der Boom verging genauso wie ein Modeboom. Was blieb, war die häufige Rekrutierung von Parlamentsmitgliedern (vorwiegend männlich), die als ,Erbabgeordnete’ in die Politik zogen. “206 Bis heute finden sich unter den Unterhausabgeordneten relativ wenig Frauen. Zwar stieg die Quote der weiblichen Kandidaten bei Unterhauswahlen von durchschnittlich 3\% in den 1980er Jahren ${ }^{207}$ auf fast 14,5\% bei der Wahl 2003 an, als 149 Frauen kandidierten. Die Chancen, gewählt zu werden, sind für Frauen (27\%) jedoch geringer als für Männer (51\%) (vgl. 3.2.2.2).

Allerdings gab es wiederum je nach Partei große Unterschiede. So versuchte vor allem die KPJ den Anteil der Frauen unter den Abgeordneten ihrer Fraktion zu erhöhen. ${ }^{208}$ Japan gilt jedoch nach wie vor als Männergesellschaft, weder in der Ministerialbürokratie noch in den Führungspositionen der Wirtschaft ist der Frauenanteil besonders hoch. ${ }^{209}$ Dieser Trend setzte sich gerade in der von paternalistischen Systemen gekennzeichneten LDP fort.

\footnotetext{
${ }^{204}$ Im Schnitt gab es alle zwei Jahre Unterhauswahlen in Japan.

${ }^{205}$ Stockwin, J.A.A.: Parties, Politicians and the Political System, in: Stockwin, J.A.A.: Dynamic and Immobilist Politics in Japan, University Press of Hawaii, Honolulu 1989, S. 40

${ }^{206}$ Derichs, Claudia; Thompson, Mark: Dynastien und politische Führerinnen in Asien. Project Discussion Paper No. 1/2003, Universität Duisburg Essen und Universität Erlangen-Nürnberg, 2003, S. 16f.

${ }^{207}$ Vgl.: Koh, Byung Chol: A.a.O., S. 99 ff.; Blechinger, Verena: Politik und Familienbande, a.a.O., S. 73.

${ }^{208}$ Vgl.: Krauss, Ellis S.: Politics and Policymakin, a.a.O., S. 41.

${ }^{209}$ Vgl.: Yoshino, Takashi; Imamura, Osamu: Nihon: Yoyaku hajimatta josei-no seiji-shinshutsu, in: Yoshino, Takashi; Imamura, Osamu,;Tanitô Etsushi (Hrsg.): Dare-ga seijika-ni naru-no-ka, Waseda Daigaku Shuppan-bu, Tôkyô 2001, S. 147-177. (Yoshino, Takashi, Imamura, Osamu: Japan: Allmählich hat das Vorrücken der Frauen in der Politik begonnen, in: Yoshino, Takashi; Imamura, Osamu; Tanitô Etsushi (Hrsg.): Wer wird Politiker?)
} 


\subsubsection{Seshûdaigishi: Erbabgeordnete in Japan}

\subsubsection{Definition und Zahl}

Die Rekrutierungs- und Karrierepfade japanischer Abgeordneter sind jedoch nicht allein durch bestimmte Berufsgruppen, einen weitgehend homogenen Bildungshintergrund oder ein hohes Einstiegsalter gekennzeichnet. Es gab und gibt, vor allem in der LDP, eine große Zahl von Abgeordneten, die in einem verwandtschaftlichen Verhältnis zueinander stehen. Auch in Deutschland oder den USA existieren Politikerfamilien, aber dass ein Viertel der japanischen Unterhausabgeordneten 1990 bereits in zweiter oder höherer Generation ein Mandat ausübte, ist im internationalen Vergleich außergewöhnlich.

Für diese Abgeordneten gibt es in Japan eine ganze Reihe von Begriffen. Einige bezeichnen die Generation, in der sie ihr Mandat ausüben, beispielsweise „Politiker der Zweiten Generation“, „Politiker der dritten Generation“ (niseigiin, sanseigiin) usw., andere bezeichnen die Herkunft aus einer politischen Familie (seiji kazoku). Am umfassendsten ist jedoch der eigentliche Terminus des „Erbabgeordneten“ (seshûdaigishi). Der in der deutschen Literatur manchmal anzutreffende Begriff „Erbpolitiker“ schließt auch die Politiker mit ein, die bereits in höherer Generation in der Politik tätig sind, aber noch kein Mandat (auf nationaler Ebene) ausüben.

Allerdings bemerkte Ichikawa Taiichi in seiner „Studie zu Erbabgeordneten“, dass dieser mit dem Wort „Erben“ verbundene Begriff die Assoziation weckt, der beschriebene Politiker sei ein direkter Nachkomme eines Abgeordneten und habe dessen Wahlkreis bzw. Basisorganisation übernommen. Ichikawas Definition von Erbabgeordneten geht jedoch sehr viel weiter. Sie umschließt genauso Parlamentarier, die Neffen, Geschwister, Enkel oder auch Adoptivsöhne von ehemaligen Abgeordneten sind. ${ }^{210}$

Adoptionen sind in japanischen Familien nicht ungewöhnlich. Kurtz spricht in diesem Zusammenhang von zwei Formen der Adoption: „First, a family without a male heir may adopt a child or young man and then promote his career through the adopted family's network and connections. Secondly, an individual may be legally adopted by a prominent family after he becomes their son in law, becoming a yoshi [Adoptivsohn - d.A.], with all rights of legal inheritance.”211 Für diese Studie ist jedoch vor allem die zweite Form der Adoption von Bedeutung.

Nach der Untersuchung von Verena Blechinger befanden sich 1996 unter den 99 Erbabgeordneten der LDP immerhin fünf Schwiegersöhne und vier Adoptivsöhne. Außerdem gab es 17 Enkel von ehemaligen Abgeordneten im Parlament, die bereits die dritte Generation von Abge-

\footnotetext{
${ }^{210}$ Ichikawa, Taiichi: A.a.O., S. 2-7.

${ }^{211}$ Vgl.: Kurtz, Donn M.: First Families in Japan, Mexico and the United States: 1946-2000, in: Comparative Sociology, Bd. 42, Nr. 5 / 2001, S. 271.
} 
ordneten darstellten. ${ }^{212}$ In seiner Studie von 1990 verzeichnet Ichikawa drei Fälle, bei denen das Mandat vom Onkel an den Neffen weitergegeben wurde. Außerdem weist Ichikawa darauf hin, dass in Politikerfamilien „nicht nur eine Kette weitergeführt wird, sondern sie sich auch zur Seite hin ausbreiten. “213 Somit zählen auch die Geschwister eines Abgeordneten, die ein Mandat auf nationaler Ebene in zweiter oder höherer Generation ausüben, zu den Erbabgeordneten. Allerdings kamen die 1990 gezählten 145 Erbabgeordneten aus 141 Politikerfamilien, d.h. es gab nur wenige Familien, die mehr als ein Familienmitglied im nationalen Parlament hatten. ${ }^{214}$

Abbildung 8: Japanische Erbabgeordnete 1994 nach Verwandtschaftsgrad

\begin{tabular}{|c|c|c|c|c|c|}
\hline & $\begin{array}{l}\text { Unterhaus- } \\
\text { abgeordneten }\end{array}$ & $\begin{array}{l}\text { Oberhaus- } \\
\text { abgeordneten }\end{array}$ & $\begin{array}{l}\text { (Premier-) } \\
\text { Ministern }\end{array}$ & Ministerinnen & Gesamt \\
\hline Söhne von & 39 & 7 & $29(3)$ & 2 & 72 \\
\hline $\begin{array}{l}\text { Schwiegersöhne } \\
\text { von }\end{array}$ & 3 & - & $4(2)$ & - & 7 \\
\hline Adoptivsöhne von & 1 & - & $5(3)$ & - & 6 \\
\hline Töchter von & - & - & $1(1)$ & - & 1 \\
\hline $\begin{array}{l}\text { Enkel (Enkelin) } \\
\text { von }\end{array}$ & 8 & $3(1)$ & $3(1)$ & - & 14 \\
\hline Gesamt & 51 & 10 & 42 (10) & 2 & - \\
\hline
\end{tabular}

(In den Klammern die in der Zahl enthaltenen Nachkommen von Premierministern.)

Nach: Blechinger, Verena: Politische Korruption in Japan, Institut für Asienkunde, Hamburg 1998, S.106.

Diese Definition führt zu einem weiteren wichtigen Punkt. Nicht alle Erbabgeordneten treten direkt die Nachfolge ihres Vorgängers an und „erben“ dessen Wahlkreis, wie die eigentliche Wortbedeutung vermuten lässt: „Wenn man das Wort „Erben“ benutzt, entsteht leicht der Eindruck, dass irgendjemand aus der Familie sofort den Eltern nachfolgt. Schaut man sich tatsächlich die Daten an, ist die Zahl der im 39. Parlament [1990 - d. A.] gewählten Erbabgeordneten, die nach dem Rücktritt der ersten Generation wie beispielsweise des Vaters direkt die Nachfolge antraten, nur halb so groß (53,1\%). “215 Auch nach der Studie von Ishibashi und Reed übernahmen nur etwa 50\% der Erbabgeordneten ihr Mandat direkt von ihrem Vorgän-

\footnotetext{
${ }^{212}$ Vgl.: Blechinger, Verena: Politik und Familienbande, a.a.O., S. 76.

${ }^{213}$ Vgl.: Ichikawa, Taiichi: A.a.O., S. 22.

${ }^{214}$ Vgl.: Ebd., S. 14.

${ }^{215}$ Ichikawa, Taiichi: A.a.O., S. 11.
} 
ger. ${ }^{216}$ Mitunter konnte nämlich zwischen Rücktritt und Nachfolge ein mehr oder weniger großer Zeitraum liegen. Nach Tanaka übernahmen allerdings 75\% der Erbabgeordneten in irgendeiner Art und Weise eine kôenkai von einem Familienmitglied. Dabei scheinen die Zahlen zumindest in den 1990er Jahren konstant geblieben zu sein. ${ }^{217}$

Außerdem übernahmen die zu den Erbabgeordneten gezählten Politiker nicht zwangsläufig den Wahlkreis ihrer Eltern. So kam es vor, dass die Abgeordneten einer anderen Partei angehörten als ihre Väter und Großväter. Ichikawa nennt in seiner Studie einige solcher Beispiele. Diese Fälle betrafen häufig, aber nicht ausschließlich, Abgeordnete, deren Vorgänger bereits vor dem Zweiten Weltkrieg ein Mandat ausübten. Nach Wahlkreisneueinteilungen und Neugründungen der Parteien waren die Nachfolger somit in anderen Wahlkreisen und Parteien tätig. Aber auch bei Erbabgeordneten, deren Vorgänger in der Nachkriegszeit ins Parlament gelangten, kam es vor, dass Zweitgenerationspolitiker in einem anderen Wahlkreis antraten. ${ }^{218}$

Tanaka Makiko, die Tochter des ehemaligen Ministerpräsidenten Tanaka Kakuei ist hierfür ein gutes Beispiel. ${ }^{219}$ Nach dem Zusammenbruch des 1955er-Systems und diversen Abspaltungen von der LDP schlossen sich viele Erbabgeordnete den neuen konservativen Parteien an und waren damit in einer anderen Partei tätig als ihre Vorgänger. Der in den Interviews befragte Abgeordnete Matsuno Yorihisa stellte solch einen Fall dar.

Die Definition der beiden Indikatoren für eine Erbfolge, „Verwandtschaft“ und „Mandat“ bzw. „öffentliches Amt“, ist also von großer Bedeutung und wird auch von Donn M. Kurtz in seiner vergleichenden Studie über Regierungs-/Staatschefs in Japan, Mexico und den USA thematisiert. Beide Indikatoren werden allerdings von Kurtz sehr weit gefasst. Während zum „öffentlichen Amt“ nicht nur die Abgeordneten des Ober- und Unterhauses, sondern „all governmental and party positions at any level of the political system as well as flag rank military officers“ ${ }^{220}$ gezählt werden, umfassen die Verwandten neben Eltern, Großeltern und Geschwistern auch Onkel, Großonkel (und -tanten) und sogar Cousins zweiten Grades. Die Definition des Verwandtschaftsverhältnisses entspricht zwar im wesentlichen der von Ichikawa Taiichi oder Verena Blechinger. Die Definition des Mandats bzw. öffentlichen Amtes ist allerdings für diese Untersuchung zu weit gefasst. Die Zahl der Abgeordneten des japanischen Unterhauses,

\footnotetext{
${ }^{216}$ Vgl.: Ishibashi, Michihiro; Reed, Steven R.: A.a.O., S. 368.

${ }^{217}$ Vgl.: Tanaka, Aiji: Nihon, in: Yoshino, Takashi; Imamura, Osamu; Tanitô, Etsushi (Hrsg.): Dare-ga Seijika-ni naru-no-ka - Kôhoshaerabi-no Kokusaihikaku, Waseda-Daigaku Shuppanbu, Tôkyô 2001, S. 21. (Tanaka, Aiji: Japan, in: Yoshino, Takashi et.al.: Wer wird Politiker? Ein internationaler Vergleich der Kandidatenauswahl.) ${ }^{218}$ Vgl.: Ebd., S. 12.

${ }^{219}$ Vgl.: Saitô, Keiko: Seikanyôran, Vol. 19, No. 45, Seisakujihô Ai Pi, Tôkyô 2001, S. 109. (Saitô, Keiko: Führer durch Politik und Bürokratie.)

${ }^{220}$ Kurtz, Donn M.: A.a.O., S. 464.
} 
die Angehörige in den Regional- und Lokalparlamenten haben, ist sehr groß. Viele Abgeordneten stammen aus Politikerfamilien, die zwar auf lokaler und regionaler, nicht jedoch auf nationaler Ebene tätig gewesen sind. ${ }^{221}$ Da die vorliegende Untersuchung sich auf die Abgeordneten des japanischen Unterhauses bezieht, macht es Sinn, die Definition des „Erbabgeordneten“ etwas enger zu fassen und sich damit der Definition der meisten anderen Studien auf diesem Gebiet anzuschließen. $^{222}$

\section{Unter diesen Gesichtspunkten bietet sich Ichikawa Taiichis Definition an, nach der ein} Erbabgeordneter ein Politiker ist, der in mindestens zweiter Generation ein Mandat auf nationaler Ebene ausübt. ${ }^{223}$

Ein kurzer Vergleich soll diese Problematik verdeutlichen: Ichikawa Taiichi zählte 1990 gemäß dieser Definition 145 Erbabgeordnete im japanischen Unterhaus, was etwa 28,4\% der Abgeordneten entspricht. Diese stammten aus 141 Politikerfamilien. ${ }^{224}$ Die Yomiuri Shinbun, die offensichtlich eine andere Definition benutzte, kam hingegen für das 39. Parlament (1990) auf 155 Erbabgeordnete, also gut 30\% der Unterhausabgeordneten. ${ }^{225}$ Andere Untersuchungen wiederum kamen zu dem Ergebnis, dass 45\% der Abgeordneten im japanischen Parlament mit ehemaligen oder noch aktiven Parlamentariern verwandt waren. ${ }^{226}$

Grundlage der Untersuchung soll aber die Definition von Ichikawa Taiichi darstellen, da seine Untersuchung zu den Standardwerken gehört und damit auch großen Einfluss auf nachfolgende Untersuchungen gehabt hat. Allerdings sollte, gerade bei neueren Zahlen, die Begriffsproblematik berücksichtigt werden.

Schlüsselt man die 141 Politikerfamilien nach Parteien auf, wird deutlich, dass das Phänomen der Erbpolitiker in dem genannten Zeitraum vor allem in der LDP zu finden war. 87,1\% der Familien waren in der LDP zu finden. In der SPJ gab es zwölf Familien, in der DSP drei und in der Kômeitô zwei. Eine weiterere Politikerfamilie gehörte dem Shaminren an, einer kurzlebigen Abspaltung der SPJ. Auch Ishibashi und Reed sind sich einig: „Second-generation Diet members and heredity seats are a phenomenon primarily, though not exclusively, of the Liberal Democratic Party (LDP), which has ruled Japan since 1955. “227

Innerhalb der LDP waren knapp über 50\% der Abgeordneten bereits in zweiter oder höherer Generation im Parlament, in der DSP etwa 21\%. In den anderen Parteien lag der Anteil der

\footnotetext{
${ }^{221}$ Vgl.: Ogawa, Tatsuyuki: Daigishi-ni kansuru kenkyû, Hyôgo Kyôiku Daigaku Daigakuin Gakuironbun, Heisei 9, http://ww1.tiki.ne.jp/ otatsu/ron/syn, Download vom 28.08.2002. (Ogawa Tatsuyuki: Eine Studie zu Abgeordneten, Diss. Hyôgo Kyôiku Daigaku, 1998.)

${ }^{222}$ Deshalb sind, wie bereits erwähnt, zwei der befragten Abgeordneten keine Erbabgeordneten im engeren Sinn.

${ }^{223}$ Vgl.: Ichikawa Taiichi: A.a.O., S. 2.

${ }^{224}$ Vgl.: Ebd., S. 14.

${ }^{225}$ Vgl.: Yomiuri Shinbun: Gekihen-no seijisentaku, a.a.O., S. 202.

${ }^{226}$ Vgl.: Bruns, Gabriele: A.a.O., S. 142.

${ }^{227}$ Ishibashi, Michihiro; Reed, Steven R.: A.a.O., S. 360.
} 
Erbabgeordneten weit unter 10\%. Diese Zahlen unterstreichen noch einmal, dass der Karrierepfad des Erbpolitikers eng mit der LDP verbunden war. ${ }^{228}$

Abbildung 9: Politikerfamilien 1980/1990 nach Parteizugehörigkeit ${ }^{229}$

\begin{tabular}{c||c|c|c|c|c} 
& LDP & SPJ & DSP & Kômeitô & Shaminren \\
\hline \hline \multirow{2}{*}{1980} & $\begin{array}{c}118 \\
(49,3 \%)\end{array}$ & $\begin{array}{c}10 \\
(9,3 \%)\end{array}$ & $\begin{array}{c}6 \\
(18,8 \%)\end{array}$ & $\begin{array}{c}1 \\
(3,0 \%)\end{array}$ & - \\
\hline \multirow{2}{*}{1990} & $\begin{array}{c}122 \\
(50,7 \%)\end{array}$ & $\begin{array}{c}12 \\
(8,6 \%)\end{array}$ & $\begin{array}{c}3 \\
(21,4 \%)\end{array}$ & $\begin{array}{c}2 \\
(4,3 \%)\end{array}$ & $\begin{array}{c}(25 \%) \\
\end{array}$
\end{tabular}

Nach: Ichikawa Taiichi: Seshûdaigishi-no kenkyû, Nihon Keizai Shinbun-sha, Tôkyô 1990, S. 14. (Ichikawa, Taiichi: Erbpolitikerforschung.)

Die Wahlkreise der Erbabgeordneten konzentrierten sich auf bestimmte Regionen Japans. Zwar gab es in über 70\% der Wahlkreise Politikerfamilien, die meisten Erbabgeordneten kamen aber aus Wahlkreisen ländlicher Regionen wie Kyûshû oder Shikoku und aus dem Umland der Metropolen wie Saitama oder Kanagawa. In diesen Regionen hatte auch die LDP ihre Hochburgen, obgleich eine LDP-Dominanz nicht automatisch eine hohe Zahl von Erbabgeordneten in einem Wahlkreis bedeutete. ${ }^{230}$

Ichikawa zeigte in seiner Studie außerdem, dass die hohe Zahl von Erbabgeordneten vor allem eine Erscheinung der Nachkriegszeit (bzw. des 1955er-Systems) war. Deren Anteil an den Unterhausabgeordneten stieg vor allem in den 1960er Jahren sprunghaft an. 78\% der Erbabgeordneten wurden nach 1963 ins Parlament gewählt. Ihre Vorgänger waren vor allem in den vier Wahlen zwischen zwischen 1946 und 1952 ins Parlament eingezogen. Geht man davon aus, dass die erste Generation dieser Politikerfamilie nach etwa 20 bis 30 Jahren zurücktrat, lässt sich der Anstieg von Erbpolitikern in den 1960er und 1970er Jahren gut erklären. Zu einem ähnlichen Ergebnis kommen Ishibashi und Reed in ihrer Studie, die ihren Fokus jedoch vor allem auf Erbabgeordneten richtet, die eine direkte Nachfolge angetreten haben. ${ }^{231}$

In der Abbildung 10 ist der Anstieg der Erbabgeordneten seit Mitte der 1960er Jahre gut erkennbar. Hier steigt die Zahl der Erbabgeordneten ab den 1960er Jahren stetig an, bis es Mitte der 1970er Jahre zu einem Einbruch kommt. Ähnliches gilt für die 1980er Jahre. Die beiden

\footnotetext{
${ }^{228}$ Vgl.: Ichikawa, Taiichi: A.a.O., S. 14.

${ }^{229}$ In Klammern der Anteil an der Gesamtzahl der Abgeordneten.

${ }^{230}$ Vgl.: Ichikawa, Taiichi: A.a.O., S. 16-20.

${ }^{231}$ Vgl.: Ichikawa, Taiichi: A.a.O., S. 11; Ishibashi, Michirhiro; Reed, Steven R.: A.a.O., S. 373ff.
} 
Wissenschaftler sprechen daher von einem „ten-year cycle“, den sie ebenfalls auf Generationswechsel zurückführen. Der Einstieg neuer Generationen vollzieht sich demnach in Wellen.

\section{Abbildung 10: Zahl der Erbfolgen (1958-1990)}

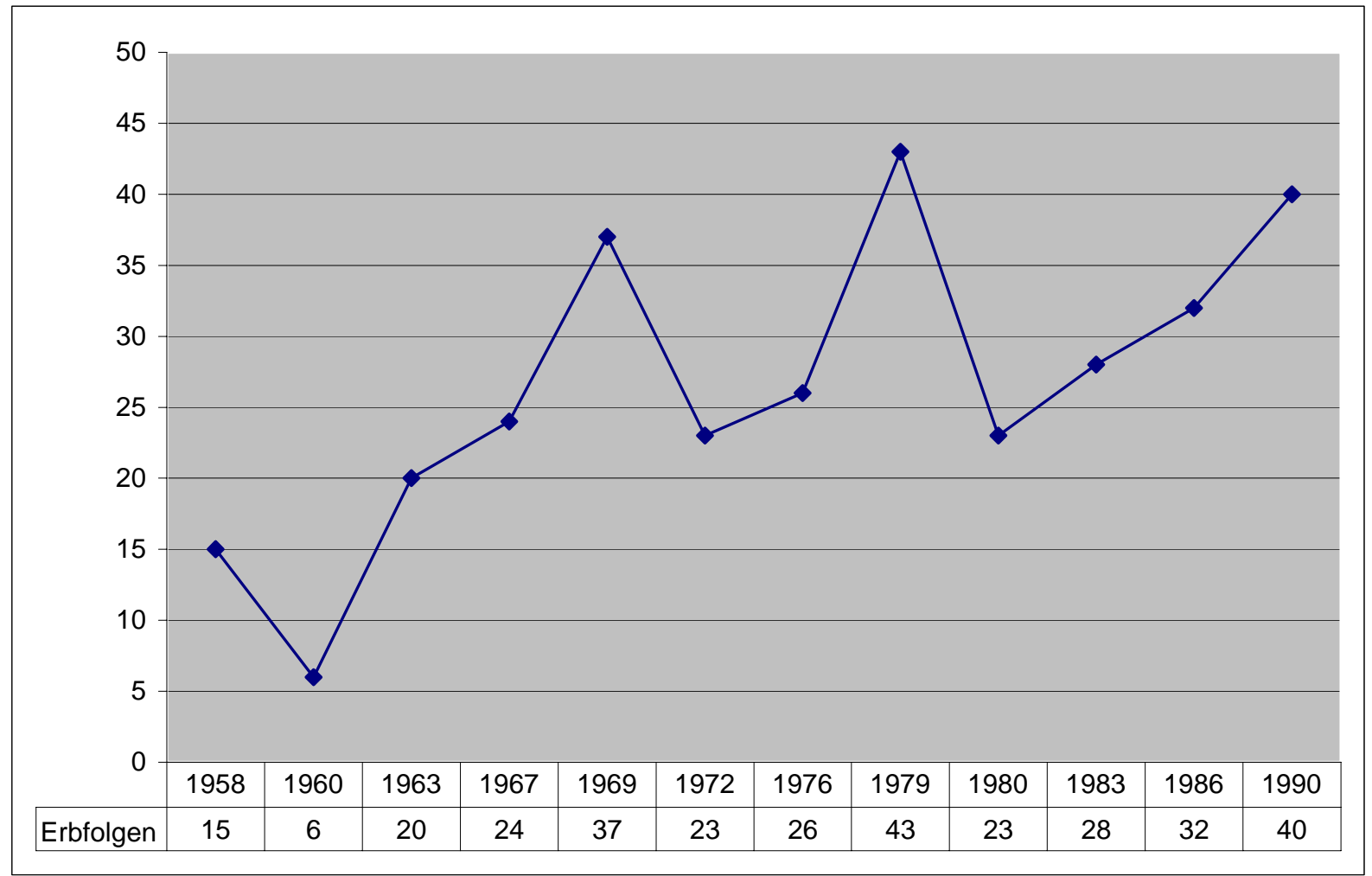

Nach: Ishibashi, Michihiro; Steven R. Reed: Second-Generation Diet Members and Democracy in Japan, in: Asian Survey, Vol. 32, No. 4/1992, S. 372.

\subsubsection{Politikerfamilien und Dynastien}

Ichikawa ordnete die Erbabgeordneten vier Gruppen zu. Gruppe I waren die Erbabgeordneten in vierter Generation, Gruppe II die Abgeordneten in dritter Generation. Erbpolitiker der zweiten Generation wurden unterteilt in Politikerfamilien, deren erste Generation vor dem Krieg in die Politik gegangen war (III-1) und Politikerfamilien, deren erste Generation nach dem Krieg begann (III-2). Die Gruppe III-2 bildete mit 78 Abgeordneten die größte Gruppe. 56\% der Erbabgeordneten insgesamt, aber fast 70\% der Zweitgenerationspolitiker stammten aus Familien, die erst nach dem Krieg in die Politik gegangen waren. Dies unterstreicht die Aussage, dass die Erbfolge vor allem ein Phänomen der Nachkriegszeit ist.

Unter den Erbabgeordneten im japanischen Unterhaus gab es eine Reihe von Abgeordneten, deren Familien über eine lange Tradition in der nationalen Politik verfügten. 26 Erbabgeordnete saßen bereits in dritter, fünf Abgeordnete in vierter Generation im Parlament. In diesem Zu- 
sammenhang wird häufig von „Politikerdynastien“ gesprochen. Doch auch hier gibt es wiederum unterschiedliche Definitionen. Einige Forscher bezeichnen mit diesem Begriff eine Familie, die auf mindestens vier Politiker mit gleichem Namen auf nationaler Ebene verweisen kann. Kurtz hingegen definiert eine Politikerdynastie als eine Familie, die über „two politically active relatives in two preceding generations with at least one of those predecessors in a major national office. “ 232 verfügt. Als Beispiel für solch eine Politikerdynastie könnte man die Familie Hatoyama nennen, die bereits in der vierten Generation Abgeordnete stellt. Mit den beiden Brüdern Yukio und Kunio sind außerdem zwei Politiker der gleichen Generation aktiv. Der ehemalige Ministerpräsident Hatoyama Yukio gründeten 1996 die Demokratische Partei Japans (DPJ), der bald darauf auch sein Bruder beitrat. (Die DPJ erhielt deshalb von den Medien zunächst den Namen „Hatoyama New Party“..) ${ }^{233}$ In diesem Sinne stellt die Familie Hatoyama eine typische Politikerdynastie dar.

\section{Abbildung 11: Japanische Erbabgeordnete 1990 nach Generation}

\begin{tabular}{l||c|c|c|c}
\multicolumn{1}{c|}{ Typ } & Familien & Abgeordnete & \multicolumn{2}{c}{ Erste Legislaturperiode } \\
\cline { 4 - 5 } & & & Vorkriegszeit & Nachkriegszeit \\
\hline I (in 4. Generation) & 4 & 5 & 4 & \\
\hline II (in 3. Generation) & 24 & 26 & 23 & 1 \\
\hline $\begin{array}{l}\text { III-1 (in 2. Generation, Vor- } \\
\text { kriegszeit) }\end{array}$ & 35 & 36 & 35 & 78 \\
\hline $\begin{array}{l}\text { III-2 (in 2. Generation, Nach- } \\
\text { kriegszeit) }\end{array}$ & 78 & 78 & & $\mathbf{7 9}$ \\
\hline \hline Gesamtzahl & $\mathbf{1 4 1}$ & $\mathbf{1 4 5}$ & $\mathbf{6 2}$ & $56 \%$ \\
\hline Verhältnis & & & $44 \%$ &
\end{tabular}

Nach: Ichikawa Taiichi: Seshûdaigishi-no kenkyû, Nihon Keizai Shinbun-sha, Tôkyô 1990, S. 14. (Ichikawa, Taiichi: Erbpolitikerforschung.)

Wie bereits gezeigt wurde, ist die Zahl der Politikerfamilien, die mehr als einen Abgeordneten (auf nationaler Ebene) stellen, nicht sehr groß. Fasst man diese sehr enge Definition einer „Dynastie“ aber etwas weiter, gibt es in Japan eine ganze Reihe von Familien, die über dynastische Merkmale verfügen. Als bekanntester Vertreter dieses Politikertypus kann sicherlich der ehemalige Ministerpräsident Hosokawa Morihiro (1993-1994) gelten. Er war Nachkomme des

\footnotetext{
${ }^{232}$ Vgl.: Kurtz, Donn M.: A.a.O., S. 468.

${ }^{233}$ Hrebenar, Ronald J.: The New Parties of the Second Party System, in: Hrebenar, Ronald J: Japan's new party system, Westview Press, Oxford 2000, S. 157.
} 
Hosokawa-Fürstengeschlechts aus Kumamoto (Kyûshû), und bereits sein Großvater mütterlicherseits war Premierminister gewesen. ${ }^{234}$ Man könnte noch einige weitere Spitzenpolitiker der dritten Generation wie Abe Shinzô oder Miyazawa Kiichi (Ministerpräsident von 1991-1993) nennen. Doch sind diese Politikerfamilien, auch wenn sie eine gewisse Tradition der Vererbung in der japanischen Politik dokumentieren, nicht repräsentativ für die Masse der japanischen Erbabgeordneten. „Diese können in der Regel nicht auf eine so lange politische „Ahnenreihe“ zurückblicken, sondern sind zu einem großen Teil Söhne von Abgeordneten, die nach 1945 als erste in ihrer Familie ein Mandat erhalten haben. “235

Man könnte zwar vermuten, dass sich im Laufe der Zeit die Zahl der Dritt- und Viertgenerationspolitiker ständig erhöhen müsste, da bei jeder Nachfolge eine Politikerfamilie entsprechend „hochgestuft“ wird. Tatsächlich ist aber die Zahl der Erbabgeordneten in dritter oder höherer Generation relativ stabil geblieben. Viele Politikerfamilien verschwanden nach der dritten oder vierten Generation wieder. Zwar wurden 1990 neun Familien „hochgestuft“, von 20 Politikerfamilien in der dritten und vierten Generation, die 1980 im Unterhaus saßen, waren aber fünf Familien nicht mehr im Parlament vertreten. ${ }^{236}$ Im Unterhaus von 1993, dem letzten nach dem alten Wahlsystem gewählten Parlament, lag die Zahl der Erbabgeordneten in dritter oder vierter Generation wieder bei $20 .^{237}$

\subsubsection{Karrierewege von Erbabgeordneten}

Innerhalb der Gruppe der Erbabgeordneten finden sich die verschiedensten Karrierewege, auch wenn die direkte Nachfolge eines Wahlkreises nach Rücktritt oder Tod des Vaters das Bild des Erbabgeordneten geprägt hat. Dennoch kann man einige typische Karrierewege bzw. Berufsstrukturen von Erbabgeordneten herausarbeiten, die sich je nach Generation stark unterscheiden. Allgemein ist es aber recht schwierig, von dem typischen Erbabgeordneten zu sprechen. Auch unter den Interviewpartnern fanden sich sehr unterschiedliche Rekrutierungswege. Hierauf wird teilweise im Rahmen der Analyse noch näher eingegangen.

Eine Untersuchung der Politikerfamilien, die bereits in dritter oder höherer Generation im Parlament saßen, zeigte, dass unter den Abgeordneten der ersten Generation viele Landbesitzer oder Unternehmer waren. Das ist nicht weiter verwunderlich, da die erste Generation bereits in der Vorkriegszeit in die Politik gegangen war. Unter den institutionellen Rahmenbedingungen der Meiji- (bis 1912) und Taishô-Zeit (bis 1925) war die politische Klasse auf Angehörige der oberen Schichten, also wohlhabende Bauern, Unternehmer etc. beschränkt. Es existierte ein

\footnotetext{
${ }^{234}$ Vgl.: Kasawara, Morio: A.a.O., S. 128.

235 Blechinger, Verena: Politik und Familienbande, a.a.O., S. 75.

${ }^{236}$ Vgl.: Ichikawa Taiichi: A.a.O., S. 14

${ }^{237}$ Vgl.: Blechinger, Verena: Politik und Familienbande, a.a.O., S. 73.
} 
Wahlrecht, das dem preußischen Dreiklassenwahlrecht sehr ähnlich war. Zwar wurde 1925 das allgemeine Männerwahlrecht eingeführt, aber bis Ende des Zweiten Weltkriegs war der Zugang zur Politik sehr beschränkt. ${ }^{238}$

In der zweiten Generation waren viele Abgeordnete vor ihrem Wechsel in die Politik als Ministerialbeamte tätig gewesen. Über 40\% der Erbabgeordneten dieser Generation zählten aber weiterhin zu der Gruppe der Landbesitzer und Unternehmer. Man kann davon ausgehen, dass in Politikerfamilien, in denen ein Unternehmen etc. weitergeführt wurde, das Vererben eines Berufes eine lange Tradition hatte. In der dritten Generation waren bereits über 40\% der Erbabgeordneten als salariman, als Angestellter in einem größeren Unternehmen tätig gewesen. Auch die Zahl der Drittgenerationspolitiker, die in der Lokalpolitik Erfahrungen gesammelt hatten, bevor sie in die nationale Politik gingen, wurde geringer. Wie bereits oben angedeutet, zogen sich viele Politikerfamilien nach der dritten Generation aus der Politik zurück, die Zahl der Familien, die in dritter oder höher Generation tätig war, blieb überraschend stabil. Ichikawa bemerkte hierzu: „Es bedeutet zwar nicht, dass eine Politikerfamilie mit der dritten Generation verschwindet, aber verglichen mit der zweiten Generation sind die Merkmale einer Politikerfamilie schwächer ausgeprägt. “239

Unter den Erbabgeordneten der Nachkriegszeit befanden sich viele Politiker, die das „politische Handwerk“ als Sekretär eines Abgeordneten gelernt hatten. In der Regel waren diese Erbabgeordneten in den Büros ihrer Vorgänger tätig gewesen. Gab es keinen geeigneten Nachfolger in der Familie, kam es vor, dass beispielsweise der Sekretär adoptiert wurde und den Wahlkreis übernahm. Andere Erbabgeordnete hatten bei befreundeten Politikern des Vaters als Sekretär gearbeitet. ${ }^{240}$

Auch wenn die Erbabgeordneten einen Beruf in der freien Wirtschaft oder in freien Berufen, etwa als Anwalt, ausübten, wurden viele dazu erzogen, später einmal den Beruf ihres Vaters zu

\footnotetext{
${ }^{238}$ Vgl.: Derichs, Claudia; Kerbo, Harold: A.a.O., S. $285 f f$.

${ }^{239}$ Ichikawa, Taiichi: A.a.O., S. 25.

${ }^{240}$ Vgl.: Moroi, Kaoru: A.a.O., S. 310.

Nicht jeder Abgeordnete stieg auf derart festen Karrierepfaden vom Sekretär seines Vaters über ein Unterhausmandat zum Ministerpräsidenten auf. Das zeigt die Karriere des ehemaligen Ministerpräsidenten Hosokawa Morihiro. Nach seinem Universitätsabschluss war er zunächst beim Asahi Shinbun-Verlag tätig. 1971 ließ er sich ins japanische Unterhaus wählen und ging zwölf Jahre später nach Kumamoto, in die Provinz, in der schon seine Vorfahren Fürsten gewesen waren. Dort wurde Hashimoto Gouverneur. 1993 saß er bereits im Unterhaus und wirkte bei der Abspaltung der Nihon Shintô von der LDP mit. In einer „Allparteienregierung“ der ehemaligen Oppositionsparteien und neuen LDP-Abspaltungen wurde Hosokawa Ministerpräsident. Dieses Beispiel zeigt, dass es bei den Erbabgeordneten unterschiedliche Karrieremuster gibt und es daher häufig schwierig ist, von dem typischen Erbabgeordneten zu sprechen. Vgl.: Uji, Toshihiko: Shushô retsuden. Itô Hirobumi kara Koizumi Junichirô made, Tôkyô Shoseki Kabushikikaisha, Tôkyô 2001, S. 352ff. (Uji, Toshihiko: Die Biographien der japanischen Ministerpräsidenten. Von Ito Hirobumi bis Koizumi Junichirô, Tôkyô 2001.)
} 
übernehmen. ${ }^{241}$ Als ideal schienen sich Karrieren zu erweisen, in denen der Erbpolitiker zunächst einige Zeit in einer Firma oder in einem Ministerium arbeitete, um später als Sekretär oder auf regionaler Ebene in die Politik einzusteigen. In jedem Fall war aber die ,jibanErfahrung“, die Erfahrung im Wahlkreis, sehr wichtig. Die Tätigkeit als Sekretär oder Lokalpolitiker ermöglichte nämlich den frühen Aufbau nützlicher Verbindungen. ${ }^{242}$

Allerdings gab es auch eine Reihe von Politikern, die sehr plötzlich eine Nachfolge antreten mussten, ohne dafür im eigentlichen Sinne „aufgebaut“ worden zu sein. Als typischer Zweitgenerationspolitiker galt beispielsweise der ehemalige Ministerpräsident Hashimoto Ryutarô (1996-1998). Hashimoto war Angestellter einer Textilfirma, als sein Vater verstarb. Im Alter von nur 26 Jahren übernahm er dessen Wahlkreis. ${ }^{243}$

Der amtierende Ministerpräsident Koizumi Junichirô musste seinen Studienaufenthalt in London abbrechen, um nach dem Tod seines Vaters nach Japan zurückzukehren. Allerdings verlor Koizumi seine erste Wahl und war danach drei Jahre als Sekretär des ehemaligen Ministerpräsidenten Fukuda tätig, bevor er schließlich ins Unterhaus gewählt wurde. ${ }^{244}$ Hier wird noch einmal die oben gemachte Aussage illustriert, dass Erbabgeordnete nicht immer sofort ihren Vorgängern nachfolgen, sondern dass es aus den verschiedensten Gründen einen zeitlichen Abstand zwischen zwei Generationen im Parlament geben kann.

Diese Beispiele zeigen ebenfalls, dass viele Erbabgeordnete relativ jung waren, als sie in die Politik gingen. Im Schnitt waren Erbabgeordnete bei ihrer ersten erfolgreichen Wahl sieben Jahre jünger als ihre Parlamentskollegen. Das Durchschnittsalter neu gewählter Abgeordneter lag 1990 bei etwa 50 Jahren. Die Erbabgeordneten unter ihnen waren im Schnitt erst 43 Jahre alt. $^{245}$ Dies stellte beim innerparteilichen Aufstieg, der nach Seniorität, d.h. Wiederwahlraten erfolgte (s.u.), einen Vorteil dar. Um beispielsweise Ministerpräsident zu werden, musste ein Abgeordneter etwa zehn Mal wiedergewählt worden sein. Das heißt, er musste etwa 25 Jahre im Parlament tätig sein, sollte aber gleichzeitig nicht älter als Mitte 60 bis Anfang 70 sein. In dieses Anforderungsprofil passten natürlich nur Politiker, die mit Anfang 40 ins Parlament gewählt worden waren. ${ }^{246}$

\footnotetext{
241 Vgl:: Kyôto Shinbun: Jiban, kanban, kaban - "sesshû" kôho 180 nin-mo, http://www.kyotonp.co.jp/kp/topics/2000jun/09/13.html, Download vom 28.08.2002. (Kyôto Shinbun: Jiban, kanban, kaban - unter den Kandidaten wieder 180 Erbpolitiker.)

${ }^{242}$ Vgl.: Ogawa Tatsuyuki: A.a.O.

${ }^{243}$ Vgl.: Kagaya, Katsuo: A.a.O., S. 187.

${ }^{244}$ Vgl.: Saitô, Keiko: Seikanyôran, Vol. 20, No. 47, a.a.O., S. 88.

${ }^{245}$ Vgl.: Ichikawa, Taiichi: A.a.O., S. 13.

${ }^{246}$ Vgl.: Blechinger, Verena: Politik und Familienbande, a.a.O., S. 84.

Die erforderlichen Wiederwahlraten für Kabinettsposten lagen weitaus niedriger (etwa 5 bis 6), ansonsten hätten bspw. Beamte, die erst mit Mitte 50 ins Parlament wechselten kaum eine Chance auf einen Ministerposten gehabt.
} 
Dementsprechend hoch war der Anteil von Erbabgeordneten unter den Regierungsmitgliedern: „Es zeigt sich, daß in jeder Regierung mindestens zwei bis drei Erbabgeordnete waren. “ ${ }^{247}$ Seit den 1960er Jahren stieg die Zahl der Erbabgeordneten, die in Regierungsposten gelangten, sprunghaft an. Von 1985 bis 1990 waren durchschnittlich 8,5 Erbabgeordnete in einer Regierung vertreten. Ichikawa zieht daraus den Schluss: „Es besteht kein Zweifel, daß Erbabgeordnete, die jung gewählt wurden, später im Kampf um die Karriere einen Vorteil besaßen. “248

${ }^{247}$ Vgl.: Ichikawa, Taiichi: A.a.O., S. 30.

${ }^{248}$ Ebd., S. 34. 


\subsection{Angebot und Nachfrage: Der Rekrutierungsprozess}

Im Folgenden soll nun zum einen geklärt werden, über welche Vorteile Erbabgeordnete bei der Kandidatenauswahl (demand-side) verfügten und welche Erwartungen es bei den Wähler und Anhängern im Wahlkreis hinsichtlich einer Nachfolge gab. Zum anderen sollen die Ressourcen, Motivationen und Fähigkeiten untersucht werden, die für Erbabgeordnete einen Karrierevorsprung darstellten, mitunter aber auch erst einen Anreiz boten, in die Politik zu gehen (supply-side). Dabei werden auch die Ergebnisse der Interviews eingearbeitet, deren Einstiegsfragen sich auf die Zeit vor 1993 bezogen.

\subsubsection{Das Selbstbild der Erbabgeordneten}

Wie bereits dargelegt wurde, findet man in der Gruppe der Erbabgeordneten höchst unterschiedliche Karrierewege. Dementsprechend unterscheiden sich auch die Selbstbilder, die die Parlamentarier von sich selbst als Erbabgeordnete haben. In vielen Fällen kann man Zusammenhänge zwischen dem eigenen Einstieg in die Politik und dem eigenen Bewusstsein als Erbabgeordneter erkennen.

Schon in der Einleitung wurde angemerkt, dass es viele Absagen von Abgeordneten gab, weil sie sich nicht als Erbabgeordnete angesprochen fühlten. Auch unter den Interviewpartner gab es, abgesehen von dem Sonderfall Terata, drei Parlamentarier, die sich nicht als Erbabgeordnete bezeichneten. Matsuno Yorihisa beispielsweise sah sich nicht in der Nachfolge seines Vaters, da er ja die Partei gewechselt hatte und seinen Wahlkreis von dem ehemaligen Ministerpräsidenten Hosokawa Morihiro übernahm: „Über mich wird in den Medien dieses Image des Erbpolitikers verbreitet. Mein Großvater war ja auch Politiker mit der gleichen kôenkai wie mein Vater. Aber bei mir ist es etwas anders, ich bin auf jeden Fall der Nachfolger von Hosokawa Morihiro. ${ }^{249}$ [...] Ich bin zwar Politiker in der dritten Generation, also Zweit- oder DrittgenerationsPolitiker. Aber weil ich die kôenkai nicht übernommen habe, denke ich, dass der Begriff Erbpolitiker nicht passt. Was heißt denn Erben? Im Japanischen hat das Erben die Bedeutung, etwas zu übernehmen. “ Auch Ôno Yoshinori nahm für sich in Anspruch, kein Erbabgeordneter im eigentlichen Sinne zu sein, da sein Vater einige Jahre vor seinem Einstieg in die Politik verstarb und er zunächst in der Regionalpolitik sein Glück versuchte.

Während in diesen Fällen die Gründe für die Ablehnung des Begriffs nachvollziehbar sind, überrascht das Selbstbild des Abgeordneten Hori, der, allerdings auf Druck der Partei und der Unterstützerorganisation, die Nachfolge seines Vaters antrat: „Ich habe das Bild eines Erbabgeordneten vor mir, wo der Vater dem Sohn sozusagen die Staffel weitergibt, nicht wahr? Aber

\footnotetext{
${ }^{249}$ Ehemaliger Ministerpräsident und Gründer der Nihon Shintô.
} 
mein Vater hatte ja eigentlich gesagt, dass ich das nicht machen soll und ich wollte es ja auch nicht. [...] Nachdem mein Vater starb, kehrte ich nach Japan zurück. Das heißt, es war meine eigene Entscheidung. Die Anhänger haben auch sehr viel dazu beigetragen. Deshalb habe ich eigentlich nicht das Gefühl ein Erbabgeordneter zu sein. “

Doch auch unter den anderen Abgeordneten, die auf sich den Begriff Erbabgeordneter anwenden ließen, gab es einige Fälle, in denen Einschränkungen gemacht wurden. Häufigstes Argument war, dass sich der eigene Fall ja doch etwas von dem eines „typischen“ Erbabgeordneten unterscheiden würde. Wobei selten konkretisiert wurde, was denn unter einem „typischen“ Fall zu verstehen sei. Als Grund wurde zumeist angeführt, dass bis zur Nachfolge, die in diesen Fällen plötzlich, z.B. nach dem Tod des Vaters, erfolgte, kein Wunsch oder Plan vorhanden war, den Wahlkreis zu übernehmen.

Auf der anderen Seite gab es wiederum Fälle (Itô, Kosaka), die einen gewissen Stolz empfanden, aus einer Politikerfamilie zu kommen. So wies der Abgeordnete Kosaka darauf hin, dass seine Familie etwas ganz besonderes sei, da es nur vier Familien gebe, die bereits in vierter Generation in der Politik aktiv sind. Itô Shintarô sprach sogar von einer Mission, die er zu erfüllen habe: „Ich bin aber in einer Politikerfamilie aufgewachsen und als mein Vater starb, überlegte ich, was wohl wäre, wenn ich nicht in die Politik ginge. Wohin wird Japan historisch gesehen gehen? Wenn man die verschiedenen Faktoren überdenkt, die eigenen Pflichten, die gegebene Situation, das Talent, musste ich diese Rolle, diese Mission, erfüllen. Das ist mein Weg. “

Vor dem Hintergrund der geführten Interviews ist dieses Selbstbild für die Auswertung von großer Bedeutung, da es wiederum die eigene politische Einstellung, z.B. in Zusammenhang mit den politischen Reformen oder der Bewertung der Rekrutierungsmechanismen, beeinflusst. Daher müssen die Selbstbilder der Erbabgeordneten bei der Bewertung der Interviews stets mitberücksichtigt werden.

\subsubsection{Die demand-Faktoren}

\subsubsection{Auswahlmechanismen in den Parteien}

Bei der Kandidatenrekrutierung gab es erwartungsgemäß große Unterschiede zwischen den Parteien. Die Oppositionsparteien verfügten mit den Gewerkschaften bzw. Sektenorganisationen der Kômeitô über typische Rekrutierungsbasen. Außerdem war bei einigen Oppositionsparteien eine gut ausgebildete Parteiorganisation vorhanden, die auch den Aufstieg über Parteiämter in die nationale Politik zuließ. In der LDP, der Partei mit den meisten Erbabgeordneten, gab es hingegen andere Mechanismen, die eingeschränkt auch für einige Oppositionsparteien galten. 
Die Kandidatenauswahl in der LDP während des 1955er-Systems ordnet Norris dem formalcentralised recruitment ${ }^{250} \mathrm{zu}$ : „[...] National party leaders, executives or faction leaders have the constitutional authority to decide which candidates are placed on the party ticket and to impose their choice on local constituencies." ${ }^{251}$ Im Fall der japanischen LDP hätten damit die Parteiführung bzw. der Parteivorsitzende und die habatsu die Rolle von main selectors übernommen.

Tatsächlich mussten sich Kandidaten von der zentralen Ebene der Partei als offizielle LDPKandidaten anerkennen lassen. Die Kandidaten wandten sich in erster Linie an die habatsu, um für die LDP in den Wahlkampf anzutreten: „A politician who wanted LDP endorsement to run for the Diet or to serve in the cabinet could not do so without joining a faction once he became a representative, and thus most LDP members became members of a faction. "252

Unter den oben genannten Bedingungen des Wahlkampfes traten die Kandidaten in erster Linie gegen Konkurrenten der eigenen Partei an. Jeder weitere Kandidat der eigenen Partei bedeutete also Konkurrenz, so dass diese Personalentscheidungen innerhalb der LDP häufig umstritten waren. ${ }^{253}$ Eine Auswahl auf nationaler Ebene war vor allem erforderlich, um die Kandidatenzahl in den Wahlkreisen „richtig“ zu bestimmen und Über- bzw. Unternominierungen zu vermeiden. Allerdings waren sowohl die Parteiführung als auch die habatsu Gruppen von Amtsinhabern, die natürlich ein Interesse daran hatten, in den Wahlkreisen möglichst wenig Konkurrenz aus der eigenen Partei zu bekommen. Daraus kann man folgern, dass wohl eher Unternominierungen ein Problem der LDP gewesen sein müsste. ${ }^{254}$

Gemessen an der Zahl der zu vergebenden Mandate war die Zahl der Kandidaten in den japanischen Wahlkreisen relativ gering. Norris’ Vergleich von 19 Ländern zeigt, dass in Japan mit 953 Kandidaten, die sich auf 512 Abgeordnetenmandate bewarben, die Rate der candidate competition am niedrigsten lag. 53,7\% der Kandidaten bekamen ein Mandat. In Ländern, in denen es ebenfalls Direktmandate gab (wenn auch Einerwahlkreise), war die Zahl der Kandidaten sehr viel höher. In Großbritannien bewarben sich 2946 Kandidaten auf 651 Mandate, d.h. es konnten 22,1\% der Kandidaten gewählt werden. Allerdings muss bei der geringen Zahl von Kandidaten in Japan auch das sehr zurückhaltende Aufstellungsverhalten der Oppositionsparteien berücksichtigt werden. ${ }^{255}$

\footnotetext{
${ }^{250}$ Vgl. Kapitel 2.2.

${ }^{251}$ Norris, Pippa: Through the Eye of the Needle, a.a.O., S. 21.

${ }^{252}$ Krauss, Ellis S.: Politics and the Policymaking Process, a.a.O., S. 46.

${ }^{253}$ Vgl.: Klein, Axel: A.a.O., S. 74.

${ }^{254}$ Vgl.: Cox, Gary W.; Niou, Emerson: A.a.O., S. 360.

${ }^{255}$ Vgl.: Norris, Pippa: Through the Eye of the Needle, a.a.O., S. 30.
} 
Die Nominierung mehrerer Kandidaten in einem Wahlkreis stellte für die Partei ein Problem dar, das man löste, indem man die Rekrutierung bzw. Aufstellung der offiziellen LDPKandidaten den habatsu überließ. „[...] It is part of the standard wisdom in Japanese politics that factions avoid running two candidates in the same district. “256 Mehrere miteinander konkurrierende Kandidaten bzw. Abgeordnete in einer habatsu konnten die Harmonie in der Gruppe empfindlich stören und reduzierten auch die Mittel, die die habatsu jedem offiziellen Kandidaten zur Verfügung stellen konnte. ${ }^{257}$

In der Regel gab es also Vereinbarungen, dass jede habatsu pro Wahlkreis einen Kandidaten aufstellte. Parteilose Kandidaten hatten die Chance, in einem Wahlkreis, in dem eine habatsu noch keinen Bewerber hatte, als Kandidat anerkannt zu werden. Außerdem kam es vor, dass parteilose Kandidaten im Erfolgsfall nachträglich in die habatsu aufgenommen wurden. ${ }^{258}$

Dennoch gab es einige Fälle, in denen eine habatsu mehrere Kandidaten aufstellte. Das ging in erster Linie auf die Kosten anderer habatsu, da es zwar eine gewisse Parteibindung der Wähler gab, jedoch keine „habatsu-Affinität“. Die Kandidaten sammelten also ihre Stimmen auch auf Kosten von Kandidaten anderer habatsu. Durch das immense Wachstum der TanakaTakeshita-ha in den 1980er Jahren stieg die Zahl von Wahlkreisen, in denen mehrere Kandidaten derselben habatsu antraten. ${ }^{259}$

Ein interessantes Beispiel für die Machtkämpfe um eine offizielle Kandidatur geben Reed und Bolland. ${ }^{260}$ In einem Wahlkreis in der Region Chiba trat einer der beiden LDPAbgeordneten zurück und zwei Mitglieder des Regionalparlaments stritten um die Nachfolge. So intervenierte die (Takeshita-)Faktion und schlug der kôenkai einen dritten Bewerber vor. Daraufhin drohte einer der beiden Regionalpolitiker, als unabhängiger Kandidat anzutreten, und versuchte die kôenkai des zurückgetretenen Abgeordneten auf seine Seite zu ziehen. Als der zweite LDP-Abgeordnete des Wahlkreises aus gesundheitlichen Gründen ebenfalls zurücktrat, nutzte der Regionalparlamentsabgeordnete diese Chance und wurde, da kein anderer Bewerber zur Verfügung stand, Nachfolger dieses Abgeordneten, der allerdings einer anderen habatsu (der Abe-ha) angehörte. Der Regionalpolitiker wurde daher gezwungen, ebenfalls in die Abe-ha einzutreten.

Die geringe Kandidatenzahl widerspricht auch nicht der Feststellung, dass es relativ wenig sichere Mandate gab, da zumeist ein relativ kleiner Umschwung im Wählerverhalten, etwa durch zusätzliche Konkurrenz aus der eigenen Partei, zum Verlust des Mandats führen konnte. Vgl.: 2.1.4.

${ }^{256}$ Reed, Steven R.; Bolland, John M.: A.a.O., S. 219.

${ }^{257}$ Vgl.: Ebd., S. 219; vgl. auch: Woodall, Brian: Japan under construction: corruption, politics and public work, a.a.O., S. $108 f$.

${ }^{258}$ Vgl.: Klein, Axel: A.a.O., S. 80.

${ }^{259}$ Vgl.: Reed, Steven R.; Bolland, John M.: A.a.O., S. 221-225.

${ }^{260}$ Vgl.: Ebd., S. 225. 
Dieses Beispiel zeigt, wie kompliziert der Prozess um die Nachfolge und die Anerkennung eines offiziellen Kandidaten in der LDP war. Weniger die Parteiführung oder der Parteivorsitzende, sondern die „Fürsprecher“ in den habatsu übernahmen die Rolle eines main selectors. An diesen Rekrutierungsmechanismen ist erkennbar, dass die Einordnung Japans in ein formell-zentralisiertes Rekrutierungssystem irreführend ist, wenn man die habatsu als Teile der informellen Politik betrachtet. Hinzu kommt, dass auf diese Weise die Rolle der kôenkai vernachlässigt wird. Hierauf wird im folgenden Abschnitt noch näher eingegangen.

Erbpolitiker konnten die genannten Mechanismen für sich nutzen. Durch ihre Vorgänger bestanden bereits gute Kontakte zu den habatsu, die an einer reibungslosen Übergabe des Wahlkreises interessiert waren. Für die habatsu-Führer war es sehr wichtig, das Mandat für die habatsu zu erhalten und die Loyalitätsverhältnisse weiterzuführen: „Die Loyalitätsverpflichtung des einzelnen Abgeordneten gegenüber seinem Faktionsführer, dessen politischer Einfluß u.a. von der zahlenmäßigen Stärke der mit ihm verbundenen Abgeordnetengruppe abhängt, beinhaltet auch das Bestreben, beim Übergang in den Ruhestand durch die Benennung eines geeigneten Nachfolgers dafür Vorsorge zu treffen, dass das Mandat für die Partei und die Faktion erhalten bleibt. “261 Erbpolitiker erwiesen sich vor allem dann als geeignete und viel versprechende Kandidaten, wenn sie bereits als Sekretär ihres Vaters oder als Lokal- und Regionalpolitiker Erfahrungen an der Basis gesammelt hatten.

Ein Großteil der LDP-Abgeordneten, die in den Interviews befragt wurden, wiesen darauf hin, dass sie zum Zeitpunkt ihres Einstiegs in die Politik von den habatsu-Führern darauf angesprochen wurden, die Nachfolge des Wahlkreises als offizieller Kandidat anzutreten. Usui Hideo gab an, dass er von dem „Lehrer“ (onshi) seines Vaters in der habatsu dazu angeregt wurde, den Wahlkreis in Chiba zu übernehmen. Der Abgeordnete Hori sagte sogar, er sei von der LDP-Führung regelrecht gezwungen worden, die Nachfolge seines Vaters anzutreten. Ähnliches gilt für Ishiba Shigeru: „Als mein Vater starb, sagte Tanaka Kakuei zu mir: ,Bitte werde Politiker.' Und weil er das sagte, tat ich das auch. Eigentlich wollte ich es gar nicht. “ Schaut man sich die Antworten der Interviewpartner aber genauer an, lässt sich allerdings feststellen, dass in der Regel bereits ein Druck seitens der kôenkai bestand, den Wahlkreis zu übernehmen, deren Rolle noch genauer besprochen wird.

Für die Erbabgeordneten selbst war letztlich die Anerkennung als offizieller Kandidat durch eine habatsu von großer Bedeutung. Zwar wäre eine Kandidatur als parteiloser Kandidat möglich und sicherlich auch nicht chancenlos gewesen, die Unterstützung durch eine Faktion bedeutete aber, wie bereits gezeigt wurde, mitunter dringend benötigte finanzielle Hilfen für den

${ }^{261}$ Blechinger, Verena: Politische Korruption in Japan, a.a.O., S. 111. 
Wahlkampf. Die Entscheidung über die Anerkennung als offizieller Kandidat der LDP war also von den Führungspersonen, d.h. von den habatsu-Führern in der LDP, abhängig. „Die Rekrutierung von Kandidaten war bisher die Sache der einzelnen habatsu, nicht wahr. Sie haben entschieden, wer der nächste Kandidat wird. Innerhalb der habatsu geschah das. Sie stellten ja so etwas wie eine Dorfgesellschaft ${ }^{262}$ dar und die Familien waren ein Teil dieses Dorfes. Das heißt, dass der Sohn, der als Sekretär arbeitete, oder auch die Ehefrau innerhalb der habatsu bekannt waren. Folglich war es sehr einfach, Nachfolger zu werden, “ so Kosaka Kenji.

\subsubsection{Die kôenkai als Selektoren}

Das oben genannte Beispiel aus der Präfektur Chiba bei der Auswahl von Kandidaten ist ein Beispiel für die Kämpfe um die Nachfolge, die es mitunter in einer kôenkai gab. Neben dem Sohn des Abgeordneten konnte es auch noch weitere Bewerber geben, die Anspruch auf eine Nachfolge erhoben, etwa die Sekretäre des Abgeordneten. Zwar konnte die habatsu auf eine Entscheidung drängen, erzwingen konnte sie eine Nachfolge aber nicht. Hier deutet sich schon die genannte Konkurrenz zwischen mehreren Selektoren an.

In den Wahlkreisen selbst übernahmen nämlich die kôenkai die Funktion eines gatekeepers oder main selectors. Sie waren es, die letztendlich über einen Kandidaten entschieden, indem sie ihn unterstützten oder ihm die Gefolgschaft verweigerten. Gerade nach der Einführung des neuen Wahlsystem traten vermehrt Fälle auf, bei denen lokale Organisation nach dem Rücktritt ihres Abgeordneten gegen die von oben erzwungene Vereinnahmung durch einen anderen Kandidaten Widerstand leisteten. Natürlich deckten sich nicht selten die Vorstellungen, die Parteiführung bzw. Faktion und kôenkai von einer Nachfolgeregelung hatten. Ein solches Beispiel stellte der Abgeordnete Hori dar, der sowohl von der Parteiführung als auch von der kôenkai regelrecht bedrängt wurde, den Wahlkreis des Vaters zu übernehmen.

Untersuchungen legen nahe, dass Erbabgeordnete auf jeden Fall einen Vorteil gegenüber anderen Bewerbern hatten, durch die Empfehlung ihres Vorgängers dessen Basisorganisation zu übernehmen. Dagegen wird allerdings eingewendet, dass Familienmitglieder nicht zwangsläufig einen Anspruch auf die Übernahme eines Wahlkreises hatten, sondern sich die kôenkai zu „Selektionsorganisationen für konservative Kandidaten““263 entwickelt.

Ishibashi und Reed kommen sogar zu dem Schluss, dass ein bereitstehender „Erbe“ aus der Familie die Konkurrenz unter möglichen Kandidaten in den kôenkai bei einer Nachfolge eher verstärkte. 1990 gab es bei einer Nachfolge, bei der kein Verwandter antrat, nur in 26\% der Fälle eine Wettbewerbssituation zwischen mehreren Kandidaten. Meldete dagegen ein Ver-

\footnotetext{
${ }^{262}$ Kosaka Kenji benutzt hier das japanische Wort mura-shakai.

${ }^{263}$ Bruns, Gabriele: A.a.O., S. 143.
} 
wandter Interesse an, gab es in 40\% der Fälle einen Wettbewerb. Es ist zu vermuten, dass es neben den Sekretären des Vorgängers noch eine Reihe anderer Aspiranten, z.B. Funktionsträger in den Unterstützerorganisationen, gab, die Anspruch auf eine Nachfolge erhoben. Diese sahen sich eventuell um die Früchte ihrer Arbeit im Wahlkreis gebracht, wenn plötzlich der Sohn des Abgeordneten zum Nachfolger gekürt wurde. Die Wettbewerbsquote allein sagt allerdings noch nichts über die Chancen von Erbpolitikern bei einer Nachfolgeregelung aus. Diese waren im Allgemeinen recht hoch. Die Wahrscheinlichkeit für Verwandte, solch einen Wettbewerb innerhalb der kôenkai zu gewinnen, lag bei ungefähr 70\%. ${ }^{264}$

Auch unter den befragten Erbabgeordneten gab es einen Fall (Kosaka), bei dem der Politiker gegen einen Rivalen in der kôenkai seines Vaters antreten musste: „[...] Es gab welche, die einen anderen Präfekturabgeordneten unterstützten und so hat die kôenkai einen Auswahlausschuss gebildet. Dann gab es dreimal Vollversammlungen. Schließlich sagte der andere Präfekturabgeordnete, dass er, anstatt mich zu bekämpfen, mich unterstützen wolle und ich konnte antreten.“

Man kann festhalten, dass für die kôenkai die Fortführung eines für beide Seiten vorteilhaften Verhältnisses von großer Bedeutung war. „Es ist zwar nicht so, daß sie [die Nachfolge des Sohnes - d.A.] nicht auch den Hoffnungen des Abgeordneten selbst entsprach, aber die Wünsche der kôenkai machten einen nicht unerheblichen Teil aus. “265 Die kôenkai wünschten natürlich einen Nachfolger, der diese Beziehungen erfolgreich weiterführte, wobei Erbpolitiker neben der Empfehlung ihres Vaters oder Onkels auch über Erfahrungen in der Wahlkreisarbeit und im Idealfall über die so wichtigen persönlichen Beziehungen verfügten. Auch hier ist die von Norris vorgenommene Einordnung in das formal-centralised recruitment problematisch, da sie die Rolle der lokalen Wahlkampforganisationen vernachlässigt.

Allerdings verschoben sich auf diese Weise auch die Ziele der kôenkai. War ihr eigentlicher Entstehungsgrund die Unterstützung eines Kandidaten, versuchten sie nun, eine Fortführung der Organisation zu gewährleisten. Ishibashi und Reed sprechen in diesem Zusammenhang von der Existenz der kôenkai als Selbstzweck, um entsprechende Vergünstigungen für ihre Mitglieder zu gewährleisten. Häufig lag es bei einem Führungswechsel nahe, einen Verwandten als Nachfolger einzusetzen. ${ }^{266}$ Der befragte Abgeordnete Hamada sagte dazu: „Vielmehr dachten die Leute um mich herum, ich würde so werden wie mein Vater. Daher waren die Erwartungen in meinem Umfeld recht groß, nicht wahr. “ Desweiteren gab Hamada zu bedenken, dass die

\footnotetext{
${ }^{264}$ Vgl. Ishibashi, Morihiro; Reed Steven R.: A.a.O., S. 376f.

265 Moroi, Kaoru: A.a.O., S. 310.

${ }^{266}$ Vgl.: Ishibashi Michihiro; Reed, Steven R.: A.a.O., S. 369.
} 
kôenkai versuchte, den Nachfolger in einer bestimmten Weise zu erziehen, um ihn zu einem erfolgreichen Vertreter ihrer Interessen zu machen.

Der japanische Politikwissenschaftler Shiratori Rei vergleicht die Erbfolge deshalb mit einem tragbaren Schrein, o-mikoshi, der bei japanischen Festen von den (jungen) Männern des Dorfes getragen wird: Während die Struktur des Schreins oder die Personen, die diesen Schrein jedes Jahr tragen, über einen langen Zeitraum gleich bleiben, wird nur der Schmuck ausgetauscht. Diesen Schmuck stellt der Abgeordnete dar und am liebsten wird ein Nachfolger ausgewählt, der keine Ambitionen hat, die Struktur der Organisation zu verändern. ${ }^{267}$

Seitens der Anhängerschaft bestand nämlich ein großes Interesse vor allem an einer reibungslosen und erfolgreichen Fortsetzung der vorhandenen Beziehungen. So konnte sich der Erbfolger mit der Bitte an die Wählerschaft wenden, anstelle des Vorgängers gewählt zu werden. Das Versprechen, die Organisation in dessen Sinne und im bisherigen Stil fortzuführen, war sicherlich nicht ohne Bedeutung. „Kurz gesagt gibt es keinen Zweifel daran, dass es unter diesem Aspekt betrachtet die Absicht des Wahlkreises ist, dass bei der Fortführung des Mandats wie in einem Familienunternehmen der ,Junior' schnell eine Nachfolge antritt und als der am besten geeignete Vertreter der Wahlkreisinteressen bereit steht - wie ein Daimyô [jap. Fürst - d. A.], der über gute Verbindungen zur Zentralregierung verfügt. “268

Für diese engen Patronagebeziehungen werden auch gesellschaftliche und traditionelle Aspekte verantwortlich gemacht, die sich in einer Service-orientierten Wählerschaft niederschlagen. Auf die einzelnen Aspekte im traditionellen Familiensystem wird später noch eingegangen. Es lässt sich aber festhalten, dass ein wenig transparentes Auswahlverfahren in der LDP Erbpolitiker genauso begünstigte wie Patronage und Klientelismus in den Wahlkreisen. Schließlich gab es für Erbabgeordnete immer noch die Möglichkeit, mit ihrer kôenkai als parteiloser Kandidat anzutreten, wenn ihnen eine Anerkennung als offizieller Kandidat versagt blieb.

Nicht alle Erbabgeordneten - und hier zeigte sich in den Interviews eine Beziehung zum Selbstbild - übernahmen eine Wahlkreisorganisation von ihrem Vorgänger. Der Abgeordnete Matsuno beispielsweise wechselte in die neu gegründete Nihon Shintô und wurde Nachfolger von Hosokawa Morihiro. Der Vater von Ishiba Shigeru dagegen war Oberhausabgeordneter und besaß keine kôenkai im eigentlichen Sinne, sondern griff im Wahlkampf auf die Unterstützerorganisationen anderer Politiker seines Wahlkreises zurück. Das führte zu einem Konflikt bei der Nachfolgeregelung: „Die einzelnen Politiker, die ihn unterstützten sagten zwar: ,Wir freuen uns sehr [, dass Du antrittst - d.A.]' Aber was in meinem Fall kompliziert war, war, dass

\footnotetext{
${ }^{267}$ Interview mit Shiratori Rei.

${ }^{268}$ Moroi, Kaoru: A.a.O., S. 310.
} 
ich Unterhausabgeordneter werden wollte [...], so dass ich gegen einen ehemaligen Unterstützer meines Vaters antreten musste, der nun mein Rivale geworden war. Es waren also nicht alle dafür. Es freuten sich also nicht alle. “ Interessanterweise bezeichnete sich Ishiba dennoch als Erbabgeordneter, obwohl er nicht die Unterstützerorganisation seines Vaters übernahm.

Alle anderen befragten Abgeordneten, wiederum mit Ausnahme des Sonderfalls Terata, übernahmen die Unterstützerorganisation ihrer Väter oder Großväter und sprachen in vielen Fällen von einem großen Druck seitens der kôenkai, den Wahlkreis weiterzuführen. Das war wahrscheinlich der Grund für einige Abgeordnete, sich nicht als „typische“ Erbabgeordnete zu fühlen, da sie ja regelrecht „gezwungen“ wurden, eine Nachfolge anzutreten.

\subsubsection{Die supply-Faktoren}

\subsubsection{Sanban: Drei Dinge, um gewählt zu werden}

Welche Ressourcen und Fähigkeiten die einzelnen Aspiranten mitbringen mussten, um als potenzieller Kandidat für einen Wahlkreis gehandelt zu werden, unterschied sich je nach Partei. Man kann aber zumindest im Fall der LDP eine Zahl von informellen Kriterien identifizieren, die Voraussetzung für eine Kandidatur waren.

Um in einer Wahl erfolgreich zu sein, so sagte man in Japan, brauchte man drei Dinge (sanban): Eine feste Basis (jiban), eine gut gefüllte Tasche (kaban) und ein bekanntes Gesicht (kanban, „Aushängeschild“). Zur Basis zählten die oben beschriebenen lokalen Netzwerke, deren Herz die kôenkai waren. Je größer und fester die Basisorganisationen des Abgeordneten waren, desto mehr Anhänger und Wähler konnte er mobilisieren und seine Stimmenzahl maximieren. Die Tasche bezeichnete zum einen die finanziellen Ressourcen, die der Abgeordnete für die Service-Schlachten verwenden konnte, zum anderen die Verbindungen (konne/connections und paipu/pipelines), über die er nach Tôkyô verfügte, um von dort „Wohltaten“ in Form von öffentlichen Aufträgen und Subventionen in den Wahlkreis zu leiten. ${ }^{269}$ Das bekannte Gesicht war aus zwei Gründen wichtig: Zum einen waren die japanischen Wahlkampfgesetze sehr streng und die Werbung in den Medien stark reglementiert. Unter diesen Bedingungen hatten es Kandidaten mit einem hohen Bekanntheitsgrad einfacher als beispielsweise „Neueinsteiger“. ${ }^{270}$ Zum anderen mussten japanische Wähler den Namen des Kandidaten selbst auf den Wahlzettel schreiben. Schon aus diesem Grund war es notwendig, sich bei den Wählern einen Namen zu machen. ${ }^{271}$

\footnotetext{
${ }^{269}$ Vgl.: Katô, Shûjirô: A.a.O., S. 86.

${ }^{270}$ Vgl.: Klein, Axel: A.a.O., S. 94ff.

${ }^{271}$ Vgl.: Blechinger, Verena: Politische Korruption in Japan, a.a.O., S. 197.
} 


\subsubsection{Ressourcen und Fähigkeiten}

Hier deutet sich bereits an, weshalb Erbpolitiker gewisse Vorteile gegenüber anderen Kandidaten hatten. Im vorangegangenen Abschnitt wurden die Mechanismen der Kandidatenrekrutierung, speziell die Nachfolge in einer kôenkai bereits erläutert. Erbpolitiker, die eine Unterstützerorganisation übernehmen konnten, hatten so einen bedeutenden Vorsprung vor anderen, neuen Kandidaten, die erst ein lokales Netzwerk aufbauen mussten. Erbpolitiker verfügten in der Regel über eine etablierte und loyale Basis, mit deren Führung sie eventuell bereits vertraut waren, z.B. als Sekretär des Vaters. ${ }^{272}$

Tatsächlich bezeichneten die Interviewpartner die Übernahme einer Wahlkreisorganisation als einen der wichtigsten Vorteile für Erbabgeordnete. Hori Kôsuke meinte dazu: „[...] Am wichtigsten ist wohl die Basis, oder? ,Jiban', ,Area' oder ,supporting force’. Also dass man tadellose Anhänger hat. In diesem Zusammenhang bedeutet es, dass, wenn Herr Satô einen Sohn hat, es für den Sohn sehr einfach ist, den Wahlkampf zu führen. “ Dieser Meinung waren auch die Interviewpartner, die keine Wahlkampforganisation von ihren Eltern übernahmen (Matsuno, Ishiba, Terata).

Die Beziehungen der Erbabgeordneten zur Bürokratie, zu lokalen Politikern und örtlichen Honoratioren, die vom Vorgänger übernommen werden konnten, waren ebenfalls nicht ohne Bedeutung. Kontakte zur (nationalen) Bürokratie sorgten beispielsweise dafür, dass öffentliche Aufträge oder Subventionen in den Wahlkreis gelangten. War der Vater eines Erbpolitikers ein erfolgreicher „Agent“ für seinen Wahlkreis gewesen, konnte der Nachfolger von diesen Verdiensten profitieren. ${ }^{273}$

Lokalpolitiker und örtliche Honoratioren waren zum einen bei der der Mobilisierung von Wählern, z.B. als Meinungsführer, wichtig. Zum anderen sorgten die Unternehmer des Wahlkreises, teilweise auch auf nationaler Ebene, für die finanzielle Unterstützung der Wahlkämpfe. Diese Praxis, die zu einigen Skandalen vor allem in der LDP führte, war auch in den Oppositionsparteien nicht unüblich. Watanabe Shû (DPJ) nannte hierfür seinen Vater als Beispiel, der Abgeordneter der SPJ war: „Aber seit der Zeit meines Vaters bildeten die Chefs von Firmen Unterstützerorganisationen und gaben selbstverständlich auch Geld. Die Spendengelder will man nicht unbedingt einem anderen geben und so ist es am besten, sie dem Sohn zu geben.“

Da eine öffentliche Politikfinanzierung fehlte, die Wahlkreisbetreuung aber, ebenso wie die Wahlkämpfe selbst, einen hohen finanziellen Aufwand bedeuteten, war eine Kandidatur in den konservativen Parteien nur möglich, wenn der Kandidat entsprechend große finanzielle Res-

\footnotetext{
${ }^{272}$ Vgl.: Tôkyô Shinbun: Dai 40 kai shûgiinsen dêtabukku, Tôkyô Shinbun Senkyohôdôhonbu, Tôkyô 1993, S. 73. (Tokyo Shinbun: Datenhandbuch zur 40. Unterhauswahl.)

${ }^{273}$ Vgl.: Hanai, Moroi: A.a.O., S. 102-103; Blechinger, Verena: Politik und Familienbande, a.a.O., S. 78-79.
} 
sourcen mitbrachte oder finanzielle Ressourcen mobilisieren konnte: „, The expectation of victory makes fundraising even easier and discourages challengers, and it becomes a self-fulfilling prophecy. “274

Außerdem konnten Spender mit einem größeren „Zeithorizont“ planen, wie Woodall bemerkt. Die Gefahr, dass ihre „Investition“ nach dem Rücktritt des Abgeordneten, abgeschrieben werden musste, war weitaus geringer, wenn mit dem Sohn ein geeigneter Nachfolger bereit stand. 275

Allerdings gab unter den Interviewpartnern in diesem Punk sehr unterschiedliche Meinungen. Einige Abgeordnete waren der Meinung, dass direkte finanzielle Vorteile für Erbabgeordnete, etwa in Form eines großen Wahlkampfbudgets, eher eine untergeordnete Rolle spielten. Allerdings wird hier wieder eine Beziehung zum eigenen Einstieg deutlich, handelte es sich doch um Abgeordnete, die wie Usui Hideo nach eigenen Angaben keine finanziellen Mittel von ihrem Vater erbten.

Dagegen wurde der finanzielle Aspekt im Zusammenhang mit den kôenkai oder dem bekannten Namen genannt. Da der Aufbau einer Wahlkampforganisation sehr teuer war, hatten Erbpolitiker finanzielle Vorteile, wenn sie eine Organisation übernehmen konnten. Auch der bekannte Name, auf den noch näher eingegangen wird, stellte eine Ressource dar, die im Wahlkampf sprichwörtlich Geld wert war. Der Abgeordnete Ishiba antwortete auf die Frage, warum es unter dem 1955er-System so viele Erbabgeordnete gab, folgendermaßen: „Der wichtigste Grund war, dass man kein Geld brauchte. Kurz gesagt, hatte man einen bekannten Namen. Ich wurde ja von Tanaka Kakuei dazu bewegt, bei der Wahl anzutreten, und Tanaka Kakuei sagte zu mir folgende Worte: ,Du hast als Politiker keinerlei Macht und auch keine Fähigkeiten. Du bist nur ein junger Mann von 27 Jahren. Du hast keinerlei Wert als Politiker. Warum trittst Du also bei der Wahl an? Der Grund ist, dass Du kein Geld brauchst!' Anders gesagt, die Leute in meinem Wahlkreis kennen alle meinen Namen. Sie kennen vielleicht nicht meinen ganzen Namen, aber sie kennen zumindest meinen Familiennamen. Den Familiennamen Ishiba kennt jeder. Das ist für gewöhnlich sehr viel Geld wert. Die guten, die fähigen Leute, die bei einer Wahl antreten, müssen sehr viel Zeit und Geld aufwenden, bis jeder ihren Namen kennt. Bei den Erbabgeordneten sind die Kosten für die Vermarktung ihres Namens gleich Null.“

Ein anderer Aspekt bei der Übernahme finanzieller Ressourcen waren die Beziehungen des Vorgängers zur nationalen Politik, bzw. zu innerparteilichen Machtgruppen. Die habatsu waren ja zum einen wichtig für die Anerkennung als offizieller Kandidat. ${ }^{276}$ Zum anderen boten sie in

\footnotetext{
${ }^{274}$ Ishibashi Michihiro; Reed, Steven R.: A.a.O., S. 369.

${ }^{275}$ Vgl.: Woodall, Brian: Japan under construction: corruption, politics and public work, a.a.O., S. 88.

${ }^{276}$ Vgl.: Ishibashi, Michihiro, Reed, Steven R.: A.a.O., S. 369.
} 
einem begrenzten Umfang finanzielle Hilfe für die Mitglieder ihrer Faktion: „The factions turned into a kind of mutual aid society for Diet members, who needed to satisfy the localized interests of their respective constituencies. The help of the faction boss was also needed for a candidate to gain official party sponsorship and for campaign speeches. „277

Japanische Erbpolitiker hatten von ihrer Herkunft nicht nur materiellen Nutzen, wie die Übernahme der kôenkai oder finanzieller Ressourcen. Abe Shinzô, ehemaliger Parteivorsitzender und Erbabgeordneter der dritten Generation, äußerte sich gegenüber einem Nachrichtenmagazin: „Ich bin zwar der Sohn von Abe Shintarô, aber ich habe die DNA von Kishi Nobosuke [Abe Shinzôs Großvater mütterlicherseits und ehemaliger Ministerpräsident - d. A.]. “278

Der Erbpolitiker nutzte damit den bekannten Namen (kanban) des Vorgängers, um sich einen Vorteil im Wahlkampf zu verschaffen. ${ }^{279}$ Die japanischen Wahlkämpfe waren nicht zuletzt durch die Konkurrenz von Kandidaten der selben Partei sehr personalisiert, die zu „ServiceSchlachten“ (sâbisu-gassen) anstelle von politischen Auseinandersetzungen führten.

Wie bereits erwähnt, war ein bekannter Name auch deshalb von Vorteil, weil die japanischen Wähler, anstatt ein Kreuz zu machen, den Namen des Kandidaten auf den Wahlzettel schreiben mussten. „Also bei der Wahl gaben die Wähler ja ihre Stimme ab, indem sie den Namen des Kandidaten aufschrieben. Mein Vater hatte ja mit dem recht einfachen Namen „Hori“ kandidiert. Die Leute in der kôenkai sind in den 35 Jahren damit vertraut geworden. Sie hatten sich daran gewöhnt, ihn zu schreiben. Wenn man in die Provinz geht, schreiben Oma und Opa den Namen einfach ein paar Mal in die Hand und gehen dann zur Wahl und schreiben auf, was sie gerade in die Hand gemalt haben. " (Hori Kôsuke)

Nicht zuletzt durch die sehr restriktiven Wahlkampfgesetze, die den Auftritt in den Medien stark eingeschränkten, hatten neue, unbekannte Kandidaten weniger Chancen, gewählt zu werden. Die Aussagen der befragten Abgeordneten stimmten deshalb in dem Punkt überein, dass ein bekannter Name für Erbabgeordnete den wichtigsten Vorteil darstellte. „In meinem Fall war es so, dass ich zwar kein Geld besaß, aber ich hatte Kanban, also den Namen meines Vaters. Das war der größte Vorteil. Daher gab es auch so viele Zweitgenerationspolitiker. “ (Usui Hideo)

Der bekannte Name muss noch unter einem weiteren Aspekt betrachtet werden. In Japan wird häufig von Wählerstimmen gesprochen, die aus Dankbarkeit oder sogar aus Mitleid (nach dem Tod des Vaters) dem Sohn gegeben werden. „Weil der Vater sich so für einen eingesetzt

\footnotetext{
${ }^{277}$ Masumi, Junnosuke: Contemporary Politics in Japan, a.a.O., S. 238.

${ }^{278}$ Yoshida, Tsukasa: Abe Shinzô. Kishi Nobosuke no DNA, ima futatabi, in. AERA, 30.12.2002, S. 67. (Tsukasa, Yoshida: Die DNA von Kishi Nobosuke, jetzt erneut.)

${ }^{279}$ Vgl.: Hanai, Hirotada: A.a.O., S. 103; Katô, Shûjirô: A.a.O., S. 86.
} 
hat, hat man das Gefühl, den Sohn unterstützen zu müssen. Auch in meinem Fall war es so. Mir wurde das auch häufig gesagt. ,Dein Vater hat wirklich viel für uns getan', sagte man dann zu mir. Jetzt höre ich es nicht mehr so oft. Aber am Anfang wurde gesagt: ,Wenn ich Dir meine Stimme gebe, denke ich dabei an Deinen Vater. '“ (Hori Kôsuke)

Problematisch ist dabei allerdings, dass die Persönlichkeit des Nachfolgers in den Hintergrund tritt. Bei der ersten Wahl mag die Abstammung zwar einen großen Vorteil darstellen. Es ist aber wichtig, sich später davon zu lösen und ein eigenes Profil zu entwickeln.

Matsuno Yorihisa hingegen machte in der neu gegründeten Partei mit seiner Herkunft aus einer Politikerfamilie eher negative Erfahrungen: „In meinem Fall habe ich nicht die kôenkai meines Vaters übernommen, aber ich hatte den bekannten Namen Matsuno. Daher gab es zunächst die Frage: ,Warum gehst Du nicht in die LDP?’ Es war anstrengend, sich ständig rechtfertigen zu müssen. “ Unter den befragten Abgeordneten war Matsuno allerdings der einzige Fall, bei dem sich der bekannte Name negativ auswirkte. Dennoch lässt sich vermuten, dass es unter anderen Erbabgeordneten, die in den 1990er Jahren in die Oppositionsparteien wechselten, weitere Beispiele gibt. Außerdem gab es sicherlich Fälle, bei denen der Name des Vorgängers z.B. durch Skandale vorbelastet war.

Die Erwartungen der verschiedenen Akteure, dass der Erbabgeordnete ähnlich wie sein Vorgänger ihre Interessen vertrat, waren also insgesamt sehr hoch. In der Tat waren viele Erbabgeordnete in die Politik hinein gewachsen und verfügten über recht große Erfahrungen in der Wahlkreisarbeit oder lokalen Politik: Die oben genannte Tätigkeit als Sekretär eines Abgeordneten findet sich im Lebenslauf vieler Erbabgeordneter. ${ }^{280}$ Dabei arbeiteten diese Erbpolitiker nicht zwangsläufig im „Betrieb“ ihres Vaters, sondern wurden mitunter auch zu befreundeten Politikern in die „Lehre“ geschickt. ${ }^{281}$ Insgesamt waren von den 131 Zweit- und Drittgenerationspolitikern des Unterhauses 199370 Abgeordnete (53,4\%) als Sekretär tätig gewesen. Davon hatten 28 Abgeordnete vor ihrer Karriere sogar bei Ministern gearbeitet. ${ }^{282}$

Aber auch auf anderen Feldern lernten Erbabgeordnete Fähigkeiten, die ihnen später in der Politik nutzen sollten. In der Regel war der Ausbildungsstand der Erbabgeordneten recht hoch, kamen sich doch aus vermögenden Familien. Das erleichterte den Einstieg in die freie Wirtschaft. Eine ganze Reihe von Politikerfamilien verfügte außerdem über Familienunternehmen (s. 5.3.). Als besonders nützlich erwies sich eine Tätigkeit in der Ministerialbürokratie. Hier vollzog sich die Rekrutierung zwar nach sehr objektiven Kriterien wie Schulbildung und Ein-

\footnotetext{
${ }^{280}$ Vgl.: Moroi, Kaoru: A.a.O., S. 310.

${ }^{281}$ Mitunter kam es auch zu einer Verbindung von zwei Politikerfamilien durch Heirat.

${ }^{282}$ Vgl.: Blechinger, Verena: Politische Korruption in Japan, a.a.O., S. 116-117.
} 
gangstests, gerade in Politikerfamilien fanden sich aber viele Abgeordnete mit einer Ausbildung an einer der Eliteuniversitäten. Kurz gesagt: Es zeigte sich, dass „über lange Jahre aufgebaute Verbindungen zur Bürokratie und Wirtschaft sehr nützlich waren. “283

In diesem Sinne sammelten Erbabgeordnete bereits in jungen Jahren Erfahrungen und wurden auf diese Weise schon in ihrer Jugend mit der Politik vertraut gemacht. So sagte der Abgeordnete Yamabana Sadao von sich: „Es war auch ein Vorteil, dass ich mit dem Bild meines Vaters und meines Großvaters vor Augen aufwuchs und die Einstellung eines Politikers lernte. “284

Die oben genannten Ressourcen und Fähigkeiten, über die Erbpolitiker verfügten, verschafften ihnen Vorteile bei der Kandidatenauswahl in den Parteien (v.a. in der LDP) und erleichterten ihnen den Einstieg in die Politik. Das zeigt sowohl das relativ niedrige Einstiegsalter von Erbabgeordneten (s. 5.3.) als auch die hohe Erfolgsquote in den Wahlen. Von 60 Erbpolitikern, die bei den Unterhauswahlen 1990 neu antraten, wurden 29 gewählt. Das entsprach einer Quote von 48,3\%. Im Durchschnitt lagen die Siegchancen von Kandidaten, die zum ersten Mal bei einer Wahl antraten nur bei 28,1\%. ${ }^{285}$ Da eine Partei in einer Wahl gewinnen will, sucht sie sich nach den genannten Kriterien ihre Kandidaten aus, wie Terata Manabu zu bedenken gab: „Warum stellte man Erbabgeordnete auf? Weil sie die Wahl gewannen! Sie hatten einen bekannten Namen und eine Basisorganisation. Damit gewannen sie die Wahl. “

\subsubsection{Motivationen und Anreize}

Der relativ einfache Einstieg in die nationale Politik und die oben genannten Vorteile, die Erbpolitiker sowohl bei der Rekrutierung als auch bei der Wahl hatten, spielten umgekehrt auch eine Rolle für die Entscheidung, in die Politik zu gehen. Die Nachfolge in einem Wahlkreis war nicht zuletzt auch deshalb attraktiv, weil Erbabgeordnete durch ihr niedriges Einstiegsalter und die vorhandenen Ressourcen und Beziehungen auch in Parlament und Partei einen Karrierevorsprung besaßen: „Those who start their career in the National Assembly at a young age, particularly second-generation politicians, are more likely to receive media attention, political contributions from groups wishing to build a relationship with an up-and-coming politician, and preferential treatment in party government appointments. Thus they have significant advantage in acquiring the funds and experience they needed to become top party leaders and eventually having a shot at the LDP presidency. “286 Die Aufstiegsmechanismen innerhalb der LDP wurden bereits ausführlich diskutiert. Dass für Erbabgeordnete die guten

\footnotetext{
${ }^{283}$ Moroi, Kaoru: A.a.O., S. 310.

${ }^{284}$ Kyôto Shinbun: Jiban, kanban, kaban - “sesshû” kôho 180 nin-mo, a.a.O.

${ }^{285}$ Vgl. Yomiruri Shinbun: Gekihen-no seijisentaku, a.a.O., S. 205.

${ }^{286}$ Kenji, Hayao: The Japanese Prime Minister and Public Policy, University of Pittsburgh Press, Pittsburgh 1993, S. $105 f$.
} 
Karrierechancen einen Anreiz darstellten, in die Politik zu gehen, kann man daher nicht von der Hand weisen.

Sicherlich machte die Übernahme einer kôenkai die Erbabgeordneten auch risikofreudiger. Da man die Organisation nicht selbst aufbauen musste und sie nun schon einmal vorhanden war, fiel es anscheinend leichter, den Schritt in die Politik zu wagen. „Zu der Zeit war mein Vater schon zurückgetreten. Aber Unterstützerorganisation war doch ein Vorteil, wie ich denke. Weil sich diese Chance ergab, dachte ich, ich versuche es einmal. " (Watanabe Shû) Die unter 2.3.3.1 genannten Vorteile stellten also ihrerseits eine Motivation dar, sich als Politiker zu versuchen.

Natürlich gab es auch Kritik, mit der Erbabgeordnete umgehen mussten: Sie hätten es nicht so weit gebracht, wenn sie nicht von ihren Vorgängern empfohlen worden wären oder deren politische Infrastruktur hätten nutzen können. Diese Kritik, die vor allem die Medien äußerten, wurde zumeist hingenommen, aber nicht weiter beachtet. In einigen Fällen stachelte sie die Erbabgeordneten geradezu an. Obuchi Yuko, zweite Tochter des ehemaligen Ministerpräsidenten Obuchi und eine der jüngsten japanischen Politikerinnen meinte dazu: „Damit man nicht sagen kann, dass Erbabgeordnete schlecht sind, strenge ich mich erst recht an. “287

\subsubsection{Verpflichtung zum Amt: Das japanische Familiensystem}

Ein weiterer Motivationsfaktor und gleichzeitig ein gewisser Zwang zur Nachfolge eines Wahlkreises lag im japanischen Familienverständnis begründet. Wie oben bereits angedeutet, gab es eine Reihe von Erbabgeordneten, die auf Druck der Familie ein Mandat übernahmen. Das japanische Familiensystem spielte als traditionelles Element in der japanischen Politik eine nicht unerhebliche Rolle: „Political inheritance has it's roots in feudal Japan, where most positions of power or social importance were passed down to an eldest son, or failing that, to sons-in-law or adopted sons." 288

Auch in anderen Berufsfeldern ist eine Erbfolge bis heute nicht ungewöhnlich. Zwar spielen bei den schriftlichen Auswahlverfahren der Ministerialbürokratie verwandtschaftliche Beziehungen nur eine geringe Rolle und einige Großunternehmen haben sogar strenge Richtlinien, die eine „Erbfolge“ im Top-Management verbieten. Dennoch ist der Anteil von höheren Beamten, deren Eltern bereits in der Zentralbürokratie arbeiteten, relativ hoch. Vor allem im klassischen japanischen Theater und in der Musik gibt es eine ganze Reihe von bekannten Künstlerfamilien. Das Prinzip der Weitergabe eines „Familienbetriebes“ findet sich ebenfalls in vielen buddhistischen Priesterfamilien, in denen der Tempel von Generation zu Generation weiterge-

\footnotetext{
${ }^{287}$ Kyôto Shinbun: Jiban, kanban, kaban - "sesshû" kôho 180 nin-mo, a.a.O.

${ }^{288}$ Do Rosario, Louise: Father's shoes. Political success becomes a matter of heredity, in: Far Eastern

Economic Review, 22.7.1993, S. 16.
} 
geben wird. ${ }^{289}$ Es gibt also einige Bereiche, in denen sich die Erbfolge in der japanischen Gesellschaft erhalten hat. Am auffälligsten ist dies jedoch in der japanischen Politik.

In diesem Zusammenhang wird oft vom japanischen Familiensystem gesprochen. Traditionell war die wichtigste Aufgabe des Haushaltsvorstandes einer japanischen Familie, den Fortbestand des Familienverbandes bzw. des Familienbetriebes sicherzustellen. Verena Blechinger verweist hier auf das Bürgerliche Gesetzbuch von 1889, in dem eine patrilineare Erbfolge vorgesehen war und alle Rechte und Pflichten vom Vater auf den ältesten Sohn übergingen. Auch politische Ämter und Verwaltungsposten wurden vererbt. „Machtpositionen etwa innerhalb eines Dorfes waren damit nicht an Individuen, sondern an bestimmte Familienverbände gebunden, deren Hausvorstände nur als Repräsentanten des Ganzen, nicht aber als Führungspersönlichkeiten aus eigener Autorität Loyalität geschuldet wurde. “290 Die bereits genannte patrilineare Erbfolge erklärt auch die geringe Zahl von Frauen unter den Erbabgeordneten. Stand nämlich kein direkter männlicher Nachfolger zur Verfügung, wurde entsprechend dem traditionellen Familiensystem außerhalb des „Haupthauses“ nach einem männlichen Nachfolger gesucht. So wurde der Familienbetrieb bzw. das Mandat an einen Nachkommen aus einer Nebenlinie, z.B. den Neffen, vererbt. Eine andere Möglichkeit war, dass beispielsweise der Schwiegersohn die Rolle des „Haushaltsvorstandes“ übernahm oder der Abgeordnete seinen Sekretär adoptierte und zum Nachfolger machte. ${ }^{291}$

Seit der Nachkriegszeit haben diese traditionell-feudalistischen Traditionen zwar an Bedeutung verloren, da sich durch die wirtschaftliche Entwicklung auch die gesellschaftlichen Bedingungen veränderten. Traditionelle Familienverbände lösten sich auf, Kernfamilien entstanden. Verena Blechinger betont aber, dass sich gerade in ländlichen Gebieten Reste dieses Familiensystems erhielten. In der Folge dieser Traditionen wurde die Übernahme eines politischen Mandats als Fortführung des Familienbetriebes betrachtet. Das galt sowohl für den potenziellen Nachfolger als auch für die Anhänger im Wahlkreis. Für Verena Blechinger gibt es daher eine weitere Erklärung für die hohe Zahl von Erbpolitikern, die sich „aus der Verknüpfung des traditionellen Familienverständnisses - und der daraus abgeleiteten Identifikation einzelner Personen mit dem gesamten Familienverband - mit dem bereits erwähnten Prinzip der Reziprozität sozialer Beziehungen in Japan “292 ergeben hat. Der Abgeordnete Itô leitete daraus sogar ein Pflichtbewusstsein gegenüber Japan ab: „Wie bei dem Sohn eines Unternehmers, der sagt: ,Ich möchte das Unternehmen noch größer machen’, gibt es Kinder von Abgeordneten, also im Fall von Unterhausabgeordneten, die sagen: ,Ich möchte Japan verbessern.'“

\footnotetext{
${ }^{289}$ Vgl.: Ichikawa, Taiichi: A.a.O., S. 16.

${ }^{290}$ Blechinger, Verena: Politische Korruption in Japan, a.a.O., S. 107.

${ }^{291}$ Vgl.: Blechinger, Verena: Politik und Familienbande, a.a.O., S. 77ff.

292 Blechinger, Verena: Politische Korruption in Japan, a.a.O., S. 109.
} 
Daneben gab es wohl auch finanzielle Gründe, das Mandat innerhalb der Familie weiterzugeben. Viele Familien wollten den Wohlstand bzw. die Position, die sie durch das politische Mandat errungen hatten, nicht aus der Hand geben und für ihre Familie erhalten. ${ }^{293}$ Da japanische Abgeordnete einen Teil ihrer politischen Aktivitäten durch Kredite finanzierten, konnte es für eine Politikerfamilie auch einen finanziellen Zwang, etwa den Druck seitens der Kreditgeber, geben, weiterhin in der Politik aktiv zu bleiben. ${ }^{294}$

Allerdings fand nicht jede Nachfolge unter dem Druck der Familie statt. Oft waren die Familienmitglieder gegen eine politische Karriere, da sie bereits mit dem Vorgänger schlechte Erfahrungen gemacht hatten. Man kann also nicht grundsätzlich von einer familiären Verpflichtung zur Übernahme des Wahlkreises sprechen. Erstaunlicherweise gab nur ein Interviewpartner, Funada Hajime an, dass seine Familie für eine Nachfolge war: „Sie meinten alle, dass ich das tun sollte. Viele waren der Meinung, dass ich, wie soll ich sagen, das Erbe meines Großvaters antreten sollte, sein Vermächtnis. “

In weiteren fünf Fällen gab es seitens der Familie keine besondere Zustimmung oder Ablehnung. In allen anderen Fällen aber berichteten die Erbabgeordneten über große Widerstände aus der Familie gegen die Entscheidung, in die Politik zu gehen. Tatsächlich fordert das Leben in der Öffentlichkeit auch gewisse private Opfer, über die Watanabe Shû sagte: „Wenn man in das Parlament einzieht, lohnt es sich zwar und man steht im Rampenlicht, aber die Familie fällt dem zum Opfer. Insbesondere die Frau muss anstelle des Abgeordneten Wahlkampf machen. Auch die Kinder werden geärgert. [...] Zum Beispiel: „Du hast Deine Tasche von unseren Steuern bezahlt!' oder ,Dein Vater macht schlimme Sachen und rafft bestimmt nur Geld zusammen.' [...] Obwohl sie so etwas nicht tun, gibt es dieses Image. Weil die Japaner denken, dass Politiker schlechte Menschen sind, werden zuerst die Familie gemobbt. Unter diesem Gesichtspunkt sagte meine Mutter zu mir: ,Lass das bitte!'“ Über ähnliche Erfahrungen konnten auch andere Interviewpartner berichten.

Allerdings muss man hier trennen zwischen der Familie im Sinne der Eltern und Familie im Sinne der eigenen Familie. Der Ablehnung der Nachfolge kam entweder von der Mutter, die ja das Politikerleben bereits durch ihren Ehemann kennen gelernt hatte, oder von Seiten der Ehefrau, da die meisten Interviewpartner bei ihrem Einstieg in die Politik bereits verheiratet waren. Insgesamt lässt sich also feststellen, dass es durchaus einen subtilen moralischen Druck geben konnte, das „Familienerbe“ weiterzuführen, der mit einem traditionellen Familienverständnis, auch auf Seiten der Wähler, zusammenhing. Dieser Druck wurde aber nicht zwangsläufig von der Familie ausgeübt. Hier gab es, wie bereits erwähnt, mitunter sogar erhebliche Wider-

${ }^{293}$ Vgl.: Moroi, Kaoru: A.a.O., S. 309.

${ }^{294}$ Vgl.: Blechinger, Verena: Politik und Familienbande, a.a.O., S. 83. 
stände. Vielmehr konnten auch die unter den demand-Faktoren genannten Interessen seitens der habatsu und der kôenkai einen moralischen Druck auf die Familie des Politikers bzw. seinen potentiellen Nachfolger erzeugen. Ob bei einer Erbfolge also ein Druck seitens der Familie, der habatsu, der kôenkai oder sogar ein eigenes „Pflichtbewusstsein“ zur Nachfolge existierte, lässt sich jedoch nur am Einzelfall prüfen. 


\subsection{The Outcome: Vorteile für Erbabgeordnete im 1955er-System?}

Die hohe Zahl von Erbabgeordneten in der japanischen Politik lässt sich vor allem auf die politischen Rahmenbedingungen des 1955er-Systems, insbesondere auf das Wahlsystem und die kôenkai, zurückführen. Unter diesem Wahlsystem, verstärkt durch die zentralistische Ausrichtung des Staates und auch einige traditionelle Einflüsse, kam es zu einer Ausrichtung der japanischen Wahlkämpfe auf persönliche Beziehungen und Patronage. Die Praxis der Politikfinanzierung begünstigte dieses System, das von den bereits genannten sanban symbolisiert wurde: „[...] Man kann nicht verneinen, daß die Kinder von Politikern einen ,Passport’ in die Politik besaßen. Dieser ,Passport' bestand aus den drei ,Insignien' des Wahlkampfes, den ,sanban'. “295

In Japan bildete sich so eine relativ geschlossene politische Klasse heraus, die auch als „Gildengesellschaft “ ${ }^{296}$ bezeichnet wurde. Wie bereits gezeigt wurde, stand diese politische Klasse nur Angehörigen bestimmter Bevölkerungsschichten offen, wobei bestimmte Bildungs- und Berufsstrukturen, vor allem aber wirtschaftliche Aspekte und persönliche Verbindungen eine große Rolle spielten. „[...] Bis zur Einführung der Einerwahlkreise gab es im wesentlichen vier Kategorien von Kandidaten, die über die Ressourcen Politiker zu werden, verfügten. Zuerst waren da die Erbabgeordneten, dann waren da die Sekretäre, also die Sekretäre, die zu Abgeordneten wurden. Dann gab es die Lokal- und Regionalpolitiker. Dazu kamen die Ministerialbeamten. Für das 1955er-System gibt es mehrere Bedeutungen, aber wenn wir die Mehrerwahlkreise mit den Einerwahlkreisen vergleichen, dann hatten die Erbabgeordneten bis zur Einführung der Einerwahlkreise einen Vorteil. “ (Itô Shintarô) Die Erbabgeordneten wurden daher als Teil einer modernen Oligarchie bezeichnet, als „Angehörige der Oberschicht in einer parlamentarischen Demokratie “297.

Die Praxis der Erbfolge war zwar nicht auf die LDP beschränkt, stellte aber ein Phänomen dar, das vor allem bei den Liberaldemokraten zu finden war. Mangelnde lokale Parteistrukturen begünstigten eine Erbfolgeregelung in den kôenkai ebenso wie die starken informellen Netzwerke in der LDP, die habatsu. Ishibashi und Reed kamen in ihrer Studie zu dem Schluss, dass der Zugang zur japanischen Politik sehr eingeschränkt war, Erbpolitikern aber Vorteile bot. Vor allem die supply-side, d.h. die Auswahlprozesse in den kôenkai begünstigten die Erbfolge: „Relatives are able to inherit seats because they are chosen by the kôenkai and for no other

\footnotetext{
${ }^{295}$ Hanai, Hirotada: A.a.O., S. 102.

${ }^{296}$ Blechinger, Verena: Politik und Familienbande, a.a.O., S. 85.

${ }^{297}$ Vgl.: Moroi, Kaoru: A.a.O., S. 309.
} 
reason. The rise in second-generation Diet members is due not to the preferences of the electorate but rather to the preferences of the selectorate." 298

Der Einfluss der habatsu, der ja eine nicht unerhebliche Rolle spielte, wird an dieser Stelle allerdings etwas vernachlässigt. Und nicht nur auf der demand-side hatten Erbabgeordnete einen Vorsprung vor anderen Kandidaten, auch auf der supply-side konnten sie zumeist größere Ressourcen vorweisen als ihre Konkurrenten. So hatten in der von Seniorität geprägten LDP Erbabgeordnete durch ihren vergleichsweise frühen Einstieg in die Politik einen weiteren Vorteil gegenüber ihren Konkurrenten. Diese Vorteile, die Erbabgeordnete bei einer Karriere vor allem innerhalb der LDP hatten, stellten ihrerseits wiederum einen gewissen Anreiz dar, in die Politik zu gehen. Viele Erbabgeordnete wuchsen in die Politik hinein und lernten, etwa als Sekretär ihres Vaters, das „politische Handwerk“. Gleichzeitig gab es oft einen gewissen Druck seitens der Familien, der kôenkai oder der habatsu, das Mandat des Vaters zu übernehmen. So reproduzierte sich die politische Klasse, wie in dem eingangs erwähnten Zitat von Verena Blechinger, tatsächlich weitestgehend selbst. Man kann in diesem Zusammenhang also tatsächlich von einer self-fulfilling prophecy sprechen.

Dennoch muss die Erbfolge unter dem 1955er-System differenziert betrachtet werden. Zum einen folgte nur ein Teil der Erbpolitiker ihren Vorgängern direkt nach. Häufig gab es, wie bereits erwähnt, einen zeitlichen Abstand zur Übernahme eines Wahlkreises durch einen Verwandten. Zum anderen gab es viele Fälle, in denen Kandidaten, die eine Erbfolge antraten, ihre Wahl verloren. ${ }^{299}$ Ishibashi und Reed arbeiten vier Bedingungen heraus, die eine erfolgreiche Erbfolge unter dem 1955er-System wahrscheinlicher machten.

Das Auftreten als offizieller Kandidat war dabei von großer Bedeutung. Nur 21\% der unabhängig angetretenen Erbpolitiker konnte ein Mandat erringen. Außerdem war entscheidend, ob der Vorgänger die letzte Wahl gewonnen hatte oder nicht. Dies klingt banal, aber 72\% der Politiker, die einem „Sieger“ nachfolgten, gewannen ein Mandat. Umgekehrt waren nur 36\% der Kandidaten erfolgreich, die das Erbe eines „Verlierers“ antraten. Drittens galt, dass ein Erbpolitiker, der bei der Nachfolge keine Konkurrenz hatte, erfolgreicher war. Schließlich gab es gewisse Zeitintervalle, die bereits erwähnten ten-year-cycles, in denen verstärkt Erbabgeordnete ins Parlament gewählt wurden. ${ }^{300}$

Neben den genannten Faktoren machte Ichikawa Taiichi auch eine gewisse Politikverdrossenheit dafür verantwortlich, dass diese Art der politischen Rekrutierung von den japanischen Wählern offensichtlich akzeptiert wurde. Umfragen in den 1980er Jahren zeigten besonders

\footnotetext{
${ }^{298}$ Ishibashi, Michihiro, Reed, Steven R.: A.a.O., S. 378.

${ }^{299}$ Vgl.: Ishibashi, Michihiro; Reed, Steven R.: A.a.O., S. 371.

${ }^{300}$ Vgl.: Ebd., S. 377f.
} 
unter japanischen Jugendlichen ein großes Misstrauen gegenüber der Politik. Der Beruf des Politikers gehörte in Umfragen zu den unattraktivsten Tätigkeiten. Auch in den Parteien, vor allem in der LDP, gab es nur wenige aktive Mitglieder. So „überließ“ man die Politik den Politikerfamilien. $^{301}$

Die Erbfolge in den Wahlkreisen wurde erst mit dem Zusammenbruch der bubble economy und dem Ende der LDP-Herrschaft in der Öffentlichkeit stärker thematisiert, als sich die porkbarrel politics unter dem 1955er-System zunehmend als Problem erwiesen. Die japanische Politik wurde zu jener Zeit von einer Reihe von Skandalen erschüttert. ${ }^{302}$ Mit den 1994 verabschiedeten politischen Reformen, vor allem des Wahlsystems und der Politikfinanzierung, war auch die Hoffnung verbunden, die nationale Politik transparenter und einem größeren Personenkreis zugänglich zu machen. ${ }^{303}$

${ }^{301}$ Vgl.: Ichikawa Taiichi: A.a.O., S. 292f.

302 Vgl.: Moroi, Kaoru: A.a.O., S. 308.

${ }^{303}$ Vgl.: Ichikawa Taiichi: A.a.O., S. 291. 


\section{Politische Reformen und Erbfolge}

\subsection{Seiji-kaikaku: Die Reformierung des politischen Systems}

\subsubsection{Der Zusammenbruch des 1955er-Systems}

Der Zusammenbruch des 1955er-Systems wurde eigentlich durch einen Machtkampf innerhalb der LDP ausgelöst. Ende der 1980er Jahre stürzte Ministerpräsident Takeshita Noboru über den so genannten „Recruit-Skandal“, in den nahezu alle führenden Politiker der LDP verstrickt waren. ${ }^{304}$ Miyazawa Kiichi, der 1991 mit Hilfe der habatsu von Takeshita Ministerpräsident wurde, versprach, innerhalb eines Jahres umfassende Reformen vorzunehmen. Dieses Versprechen wurde aber von Miyazawa bald wieder relativiert. Die Reformer innerhalb der Partei waren enttäuscht und warfen dem Ministerpräsidenten Wortbruch vor. Auch Wirtschaftskreise übten massive Kritik an der Verschleppung überfälliger Reformen. ${ }^{305}$

Ein neuer Skandal, die „Sagawa-Kyûbin-Affäre“, erschütterte wenig später die LDP. Eine Transportfirma hatte eine große Zahl von Abgeordneten - angeblich sollen es über 100 gewesen sein - bestochen, um eine Gesetzesliberalisierung zu erreichen. Allein Kanemaru Shin, einer der führenden Politiker in der Takeshita-Gruppe, hatte 500 Mio. Yen erhalten und zog sich auf öffentlichen Druck aus der Politik zurück. ${ }^{306}$ Daraufhin kam es zu Nachfolgekämpfen in der habatsu. Es bildeten sich zwei Gruppen, eine um den reformfreudigen Ôzawa Ichirô und eine um den eher reformkritischen Obuchi Keizô. Als Obuchi den Machtkampf für sich entscheiden konnte, gründete Ôzawa zusammen mit dem als Reformer geltenden Politiker Hata Tsutomu das „Reformforum 21“ und forderte neben einem „Generationswechsel“ in der LDP auch eine Reformierung des politischen Systems. Die Oppositionsparteien sahen nun eine Gelegenheit, mit Hilfe dieser Gruppe die Regierung Miyazawa zu stürzen. ${ }^{307}$

Trotz der offensichtlichen Machtkämpfe in der LDP, spricht Kato den sich abspaltenden Abgeordneten politische Ziele nicht ab: „In summary, the LDP was perceived to have deteriorated by certain members. They believed the party would become paralyzed by political corruption and with special interest groups, and they advocated political reform. „308

Am 18. Juni 1993 wurde im Unterhaus ein Misstrauensvotum gegen den Ministerpräsidenten eingebracht. Unter der Führung von Hata Tsutomu stimmten 39 LDP-Abgeordnete gegen Miy-

\footnotetext{
${ }^{304}$ Vgl: Blechinger, Verena: Politische Korruption in Japan, a.a.O., S. 269ff.

${ }^{305}$ Vgl.: Klein, Axel: A.a.O., S. 123.

${ }^{306}$ Vgl.: Farley, Maggie: Japans Press and the Politics of Scandal, in: Farley, Maggie / Krauss, Ellis S.: Media and Politics in Japan, University of Hawaii Press, Honolulu, 1996, S. 151ff.

${ }^{307}$ Vgl.: Klein, Axel: A.a.O., S. 129-137; Blechinger, Verena: Politische Korruption in Japan, a.a.O., S. 328.

${ }^{308}$ Kato, Junko: When the Party Breaks Up: Exit and Voice among Japanese Legislators, in. American Political Science Review, Vol. 93, No. 4/1998, S. 861.
} 
azawa. Der Ministerpräsident musste zurücktreten und es wurden Neuwahlen angesetzt. Ôzawa und Hata gründeten die Shinseitô (Erneuerungspartei, JRP), deren Gründungsmanifest bezeichnenderweise eine Entschuldigung beim japanischen Volk enthielt, „zur Einparteienherrschaft der LDP beigetragen zu haben“. ${ }^{309}$

Bei den folgenden Wahlen verfehlte die LDP zum ersten Mal seit 38 Jahren die absolute Mehrheit im Unterhaus, blieb aber stärkste Fraktion. Auch die SPJ musste große Verluste hinnehmen. Eine Koalition von acht Parteien wählte den Führer der Nihon Shintô Hosokawa Morihiro zum neuen Ministerpräsidenten. ${ }^{310}$ Das 1955er-System war zerbrochen.

Hosokawa trat als „Mr. Clean“ an, um die japanische Politik von Korruption zu säubern. Er bildete eine Koalitionsregierung, der sieben Parteien angehörten. Die LDP fand sich zum ersten Mal seit 38 Jahren in der Opposition wieder. Die unter seiner Regierung erarbeiteten ReformEntwürfe wurden zwar weitgehend angenommen, das Inkrafttreten der Gesetze jedoch auf unbestimmte Zeit verschoben. Hosokawa musste nach wenigen Monaten wegen eines nicht zurückgezahlten „Kredits“ der Firma Sagawa Kyûbin gehen. ${ }^{311}$ Auch der Minderheitsregierung unter Hata Tsutomu war keine lange Dauer beschieden, sie brach nach wenigen Monaten zusammen. In einer „großen Koalition“ mit der SPJ, die mit Murayama Tomiichi zum ersten Mal seit 46 Jahren den Ministerpräsidenten stellte, kehrte die LDP in die Regierung zurück. ${ }^{312}$ Nur zwei Jahre später zerbrach auch diese Koalition.

\subsubsection{Das neue Parteiensystem}

Bemerkenswert am Zusammenbruch der 38-jährigen LDP-Herrschaft ist, dass der Regierungswechsel nicht durch einen Wahlsieg der Opposition herbeigeführt wurde. „It seems as though the only way the LDP would lose its grip on the Diet was by organizational suicide. That is exactly what happened in 1993."313 Das wurde nicht zuletzt daran deutlich, dass die Faktionskämpfe, die bisher weitgehend innerhalb der Partei stattgefunden hatten, nun auch in der Öffentlichkeit ausgetragen wurden. ${ }^{314}$

Im Oppositionslager verschob sich das Kräftegleichgewicht. Die SPJ, die sich nun SocialDemocratic Party of Japan (SDPJ) nennt, verlor ihre Bedeutung als größte Oppositionspartei. Viele Wähler verziehen ihr nicht die „große Koalition“ mit der LDP und die Verschleppung

\footnotetext{
${ }^{309}$ Vgl.: Der Spiegel: Ende der Verläßlichkeit, in: Der Spiegel, Nr. 26/1993, 28.06.1993, S. 138-139; Der Spiegel: Tiefes Bedauern, in: Der Spiegel, Nr. 28/1993, 12.07.1993, S.124.

${ }^{310}$ Vgl.: Der Spiegel: Revolution von oben in: Der Spiegel, Nr.30/1993, 26.07.1993, S. 117.

${ }^{311}$ Vgl.: Der Spiegel: Abgang des Saubermannes, in: Der Spiegel Nr. 15/1994, 11.04.1994, S. $138 f$.

${ }^{312}$ Foreign Press Center Japan (Hg.): The Diet, Election and Political Parties. “About Japan” Series, Nr. 13, 3. Aufl., Foreign Press Center Japan, Tôkyô 1995, S. $124 \mathrm{ff.}$

${ }^{313}$ Hrebenar, Ronald R.: The Changing Postwar Party System, in: Hrebenar, Ronald J: Japan’s new party system, Westview Press, Oxford 2000, S. 29.

${ }^{314}$ Vgl.: Jain, Purnendra C.: Party Politics at the Crossroads, in: Jain, Purnendra C.; Inoguchi, Takashi: Japanese Politics Today. Beyond Karaoke Democracy? St. Martin’s Press, New York 1997, S. 20
} 
einiger Reformvorhaben. ${ }^{315}$ Als weiterer Grund für den Niedergang der SDPJ wird die Zersplitterung in linke und rechte Kräfte angeführt. Während ihrer Regierungszeit unter Ministerpräsident Murayama gab sie viele ihrer früheren Positionen, etwa ihre ablehnende Haltung gegenüber den Streitkräften, auf und verlor so einen großen Teil ihrer Kernwählerschaft. 1996 erhielten die Sozialdemokraten erstmals weniger Stimmen als die Kommunisten. ${ }^{316}$

Die SDPJ-Fraktion im Unterhaus litt außerdem an zahlreichen Aus- und Übertritten ihrer Parlamentarier. Innerhalb der SDPJ hatten sich die Gegensätze zwischen linkem und rechtem Lager verschärft. Die rechten Kräfte gründeten mit der Shinminren (Neue Demokratische Liga) eine innerparteiliche Gruppierung, die 60 Abgeordnete umfasste und deren Mitglieder nach den hohen Verlusten in der Wahl 1996 zur Shinshintô übertrat. ${ }^{317}$

Aus den Abspaltungen der LDP bildeten sich ebenfalls neue Parteien. Zwar hatte es vor 1993 bereits LDP-Abspaltungen wie den New Liberal Club (NLC) gegeben, aber diese konservativen „Mini-Parteien“ hatten im 1955er-System nur eine untergeordnete Rolle gespielt.

\section{Abbildung 12: Entwicklung der japanischen Parteien ab 1992 (vereinfacht)}

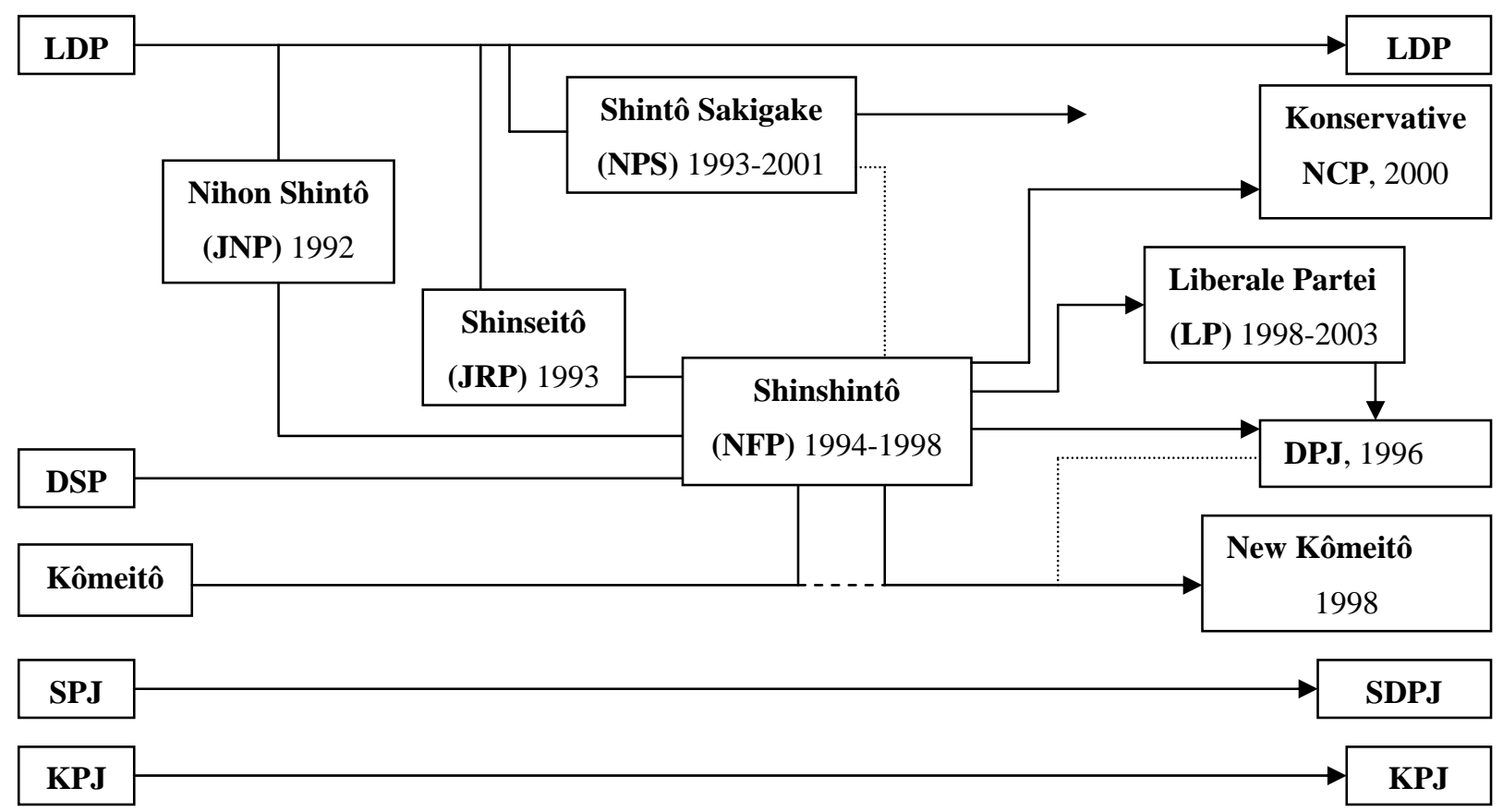

Nach: Foreign Press Center Japan (Hg.): Changing Japanese Politics. “About Japan” Series, Nr. 26, 2. überarb. Aufl., Foreign Press Center Japan, Tôkyô 2003, S. 116.

\footnotetext{
${ }^{315}$ Vgl.: Hrebenar, Ronald J.: The Liberal Democratic Party: Still the Most Powerful Party in Japan, in: Hrebenar, Ronald J: Japan's new party system, Westview Press, Oxford 2000, S. 90-94

${ }^{316}$ Stockwin, J.A.A.: The Socialist Democratic Party (Formerly Japan Socialist Party): A Turbulent Odysse, in: Hrebenar, Ronald J: Japan’s new party system, Westview Press, Oxford 2000, S. 230f.

${ }^{317}$ Vgl.: Kato, Junko: When the Party Breaks Up: Exit and Voice among Japanese Legislators, a.a.O., S. 865ff.
} 
Neben der oben genannten Shinseito (JRP) von Ozawa und Hata existierte bereits seit 1992 die Nihon Shintô (JNP), die von dem ehemaligen LDP-Abgeordneten Hosokawa Morihiro gegründet worden war. Um diese beiden Parteien herum gründete sich 1994 die Shinshintô (NFP), der auch Teile der DSP beitraten. ${ }^{318}$ Die NFP bildete also eine Art Sammlungsbewegung unterschiedlicher politischer Kräfte, schaffte es jedoch nicht, die einzelnen heterogenen Gruppierungen zu integrieren. Zwischen einigen Akteuren bestanden sogar persönliche Feindschaften. ${ }^{319}$ Nach nur vier Jahren löste sich die Shinshintô auf, Liberale Partei (LP) und Neue Konservative Partei (NCP) entstanden

Zur Unterhauswahl 1996 waren auch die Kômeitô-Kandidaten auf nationaler Ebene für die Shinshintô angetreten. Der Shinshintô waren die neuen Mitglieder wegen ihrer gut organisierten Wählerbasis sehr willkommen. Auf lokaler Ebene existierte die Kômeitô allerdings weiter. Zwar gab es auf lokaler Ebene Kooperationen mit den Kandidaten der Shinshintô, die Parteiorganisationen waren jedoch zumeist voneinander getrennt. Als die Shinshintô zerfiel, wurde nach einem kleinen Zwischenspiel als „Neue Friedenspartei“ die Kômeitô reorganisiert. Heute nimmt die Partei abgesehen von ihren religiösen Zielen eine gemäßigt konservative Position ein. $^{320}$

Während die Shinshintô (NFP) als konservative Alternative zur LDP scheiterte, war eine andere Neugründung, die Demokratische Partei Japans (DPJ, Minshutô), wesentlich erfolgreicher. Mit ihrem Zugpferd, dem angesehenen ehemaligen Gesundheitsminister Kan Naoto, versuchte die DPJ, sich als dritte Kraft neben LDP und NFP zu etablieren. Ihre programmatischen Defizite konnte sie durch die hohe Zahl junger Kandidaten ausgleichen. Nach Auflösung der NFP traten viele Abgeordnete der DPJ bei. Die DPJ hat sich zur zweitgrößten Partei nach der LDP entwickelt. ${ }^{321}$ Ende September 2003 vereinigten sich schließlich die DPJ und die von Ozawa geführte Liberale Partei (LP) unter dem Namen der DPJ. Ein entsprechendes Angebot wurde, allerdings erfolglos, auch an die SDPJ gerichtet. ${ }^{322}$

Die vielen Parteifusionen brachten für die DPJ jedoch einige Probleme mit sich. Die Partei gilt als sehr heterogen und innerlich zerrissen. Die Parteiführung steht bis heute vor der Herausforderung, eine ganze Reihe unterschiedlicher politischer Gruppierungen integrieren zu

\footnotetext{
${ }^{318}$ Vgl.: Hrebenar, Ronald R.: The New Parties of the Second Party System, a.a.O., S. 149ff.; Klein, Axel: A.a.O., S. 134, $191 \mathrm{ff}$.

${ }^{319}$ Vgl.: Kataoka, Masaaki: Changing local Politics: Party Realignment and growing Competition, in: Jain, Purnendra C.; Inoguchi, Takashi: Japanese Politics Today. Beyond Karaoke Democracy? St. Martin’s Press, New York 1997, S. 211f.

${ }^{320}$ Vgl.: Hrebenar, Ronald J.: The Komeito Returns: The Party of „Buddhist Democracy“, a.a.O., S. 198ff.; Inoguchi, Takashi: A Step Toward One-Party Predominance: Japan’s General Election of 20 October 1996, in: Government and Opposition, Vol. 32, No. 1/1997, S. 53.

${ }^{321}$ Vgl.: Bruns, Gabriele: A.a.O., S. 235ff.

${ }^{322}$ Vgl.: Nihon Keizai Shinbun: Jiyû, kaihô shi gôryû, Nihon Keizai Shinbun, 24.7.2003, S. 1. (Nihon Keizai Shinbun: Liberale: Befreiender Zusammenschluss)
} 
müssen. Interne Machtkämpfe schwächen, bei allen Erfolgen, die die Partei bisher hatte, ihre Popularität. Die DPJ scheint für viele Wähler noch keine überzeugende (Regierungs-) Alternative zur LDP darzustellen. Nach Köllner ${ }^{323}$ hängt die Zukunft der DPJ in hohem Maß von den Entwicklungen in der LDP ab. Im Kapitel über die Zukunft der Erbfolge wird auf diesen Punkt noch näher eingegangen.

Die LDP konnte nach den Umbrüchen Anfang der 1990er Jahre bald wieder in die Regierung zurückkehren. Dass die Liberaldemokraten ihren Rückhalt in der Bevölkerung größtenteils wiedererlangte, wird auch mit der Enttäuschung vieler Wähler über die „Reformparteien“ erklärt, die während ihrer Regierungszeit nur wenige Erfolge vorweisen konnten. ${ }^{324}$

\subsubsection{Die Reformdiskussion: Ziele und Konflikte}

Die Diskussion um eine grundlegende Reformierung des politischen Systems reicht weit zurück. Bereits im Jahr 1973 ließ Ministerpräsident Tanaka Kakuei eine Gesetzesvorlage zur Umgestaltung des Wahlsystems erarbeiten, die allerdings an verschiedenen Widerständen, nicht zuletzt aus der Opposition, scheiterte. Diese Pläne, die ein reines Mehrheitswahlsystem und die Einführung von Einerwahlkreisen vorsahen, wurden von der Regierung Kaifu und schließlich von der Regierung Miyazawa fortgeführt. Die Mehrerwahlkreise standen deshalb im Mittelpunkt der Reformbemühungen, weil sie als Hauptursache für die kostenintensiven Wahlkämpfe und die politische Korruption sowie den geringen politischen Wettbewerb zwischen den Parteien betrachtet wurden. ${ }^{325}$

Wie bereits unter 1.1.2 beschrieben, sind historisch gesehen Reformen unter äußerem Druck weit weniger erfolgreich als die, die dem Eigeninteresse der politischen Klasse entsprechen. ${ }^{326}$ Unter diesem Aspekt lässt sich auch die Diskussion über die politischen Reformen in Japan betrachten. Die Diskussion um die Veränderung des Wahlsystems war nämlich auf der einen Seite von der Angst der Mandatsträger vor dem Verlust ihrer Mandate geprägt, etwa durch Neueinteilung der Wahlkreise oder Wegfall von Direktmandaten durch die Einführung eines „Grabensystems“ (s.u.). Auf der anderen Seite kämpften kleinere Gruppierungen für ein proportionales Wahlsystem, um ihre Wahlchancen zu erhalten bzw. auszubauen. Als Alternative zum SNTV-MMD-Wahlsystem wurden im Wesentlichen zwei Möglichkeiten diskutiert:

\footnotetext{
${ }^{323}$ Vgl.: Vgl.: Köllner, Patrick: Zwischen Konflikt, Kohäsion und parteiübergreifender Kooperation: Probleme und Perspektiven der Demokratischen Partei Japans, in: Japan aktuell. Wirtschaft. Politik. Gesellschaft, Heft Februar/2003, S. 62f.

${ }^{324}$ Vgl.: Inoguchie, Takashi: A Step Toward One-Party Predominance: Japan's General Election of 20 October 1996, a.a.O., S. 55.

${ }^{325}$ Vgl.: Seligmann, Albert L.: Japan’s new electoral system, in: Asian Survey, Vol. 37, No. 5/1997, S. 412f.

${ }^{326}$ Vgl.. Borchert, Jens: Die politische Klasse in westlichen Demokratien, a.a.O., S. 32.
} 
1. Durch die Einführung eines reinen Mehrheitswahlsystems mit etwa 500 Einerwahlkreisen prophezeite man die Entstehung eines Zweiparteiensystems, in dem die lokalen Parteiorganisationen die Möglichkeit hätten, einen Kandidaten gezielt zu unterstützen. Auf diese Weise würde auch der Unterhalt persönlicher Unterstützerorganisationen überflüssig werden.

Vorwahlen nach US-amerikanischer Art sollten dabei den Wählern die Möglichkeit geben, auf die Kandidatur Einfluss zu nehmen. Damit würde auch die Erbfolge in der japanischen Politik verschwinden und die Qualität des politischen Personals steigen. ${ }^{327}$ Diese Pläne wurden vor allem von kleineren Oppositionsparteien bekämpft, die sich dadurch in ihrer Existenz bedroht sahen und stattdessen die Einführung eines Verhältniswahlsystems forderten.

2. Ein Grabensystem, in dem etwa 60\% der Sitze durch Mehrheitswahl in Einerwahlkreisen und 40\% der Sitze durch Verhältniswahl bestimmt werden sollten, war von der Regierung Kaifu 1991 erarbeitet worden und stellte einen Kompromiss zwischen verschiedenen Reformvorschlägen dar. Durch die Einführung eines Verhältniswahlsystems erhofften sich die Reformer eine Stärkung der Parteien bei der Kandidatenaufstellung. Der programmatische Wettbewerb zwischen Kandidaten unterschiedlicher Parteien sollte gefördert und „Service-Schlachten“ vermieden werden. ${ }^{328}$

Ein reines Verhältniswahlsystem wurde hingegen nicht in Betracht gezogen. Klein macht hierfür politische Traditionen in Japan verantwortlich: „Wie zahlreiche Umfragen bestätigen, bevorzugen japanische Wähler personenbezogene Stimmgebungsverfahren, weshalb das Wahlsystem des Oberhauses nach seiner Reform 1982 häufig Kritik ausgesetzt war. “329 Auch mit der Gefahr instabiler Koalitionsregierungen wurde gegen ein reines Verhältniswahlsystem argumentiert.

Die Ereignisse des Jahres 1993, als die Miyazawa-Regierung zerbrach, lassen sich auch unter dem Aspekt der politischen Reformen betrachten. Da die LDP zu diesem Zeitpunkt auf die Stimmen der Opposition im Oberhaus angewiesen war, nahm man Verhandlungen über eine Kompromisslösung in Form eines Grabensystems auf. ${ }^{330}$ Dieser Prozess wurde aber von den Politikern des „Reformforums 21“ sabotiert. Zum einen versuchte man so, die Regierung Miyazawa zu destabilisieren und einen Machtwechsel in der Partei herbeizuführen, zum anderen

\footnotetext{
${ }^{327}$ Vgl.: Ichikawa Taiichi: A.a.O., S. 291-293.

${ }^{328}$ Vgl.: Blechinger, Verena: Auf dem Weg zu „sauberer Politik“ und transparenten Strukturen? A.a.O., S. 165166.

${ }^{329}$ Klein, Axel: A.a.O., S. 146.

${ }^{330}$ Vgl.: Blechinger, Verena: Politische Korruption in Japan, a.a.O., S. 333.
} 
gab es innerhalb der LDP zahlreiche Interessenkonflikte. Der Plan der LDP-Führung sah nämlich vor, dass in den neu zu schaffenden Einerwahlkreisen die jüngeren Abgeordneten der LDP als Kandidaten verbleiben und die älteren Abgeordneten als attraktive „Zugpferde“ auf der Parteiliste antreten sollten. Dies rief den Widerstand so genannter midcareer incumbents ${ }^{331}$ hervor, deren Karrierechancen dadurch beträchtlich sanken. Diese Angst vieler Parlamentarier vor dem Verlust ihres Mandats wussten Ôzawa und seine Anhänger für sich zu nutzen: „Für ihn und die Politiker in seinem Umkreis war das Misstrauensvotum eine Chance, die Herrschaft der LDP unter der Führung alter Männer aus der Taishô-Zeit (Geburtsjahrgang 1912-1926) zu beenden und einen Macht- wie auch einen Generationswechsel herbeizuführen. “332

Die Diskussion um politische Reformen macht deutlich, dass die Auseinandersetzungen nicht nur von der Furcht vieler Abgeordneter vor dem Verlust des eigenen Wahlkreises geprägt, sondern ebenso von Machtkämpfen innerhalb der LDP. ${ }^{333}$ Durch die Änderung des Wahlsystems erhoffte man sich deshalb auch die Beseitigung der habatsu. PARC-Vorsitzender Matsuno Raizô ${ }^{334}$ argumentierte, , the party factions would never disappear as long as the present mediumsized constitueny system is operating. “335 Indirekt erwartete man wohl eine Stärkung der Partei als solcher, da sie durch die habatsu geschwächt wurde, deren Mitglieder sich ja eher der Faktion als der Partei im Ganzen verpflichtet fühlten. ${ }^{336}$

Vorangetrieben wurden die Reformen vor allem von den neu gegründeten politischen Gruppierungen, die 1993 entstanden waren, wie etwa die von Hosokawa Morihiro gegründete Nihon Shintô. ${ }^{337}$ In den Medien stieß die Reformdebatte auf reges Interesse. Blechinger schreibt den Kräften um Hosokawa den Verdienst zu, das Thema Reformen in der öffentlichen Debatte vorangetrieben zu haben. ${ }^{338}$

So überrascht es kaum, dass es die Koalitionsregierung unter Ministerpräsident Hosokawa war, die schließlich ein Reformpaket auf den Weg brachte, das neben einem neuen Wahlsystem auch eine öffentliche Parteienfinanzierung vorsah. Interessanterweise gab es vor allem beim

\footnotetext{
${ }^{331}$ Woodall, Brian: The Politics of Reform in Japan’s Lower House Electoral System, in: Grofman, Bernard et.al. (Hg.): Elections in Japan, Korea and Taiwan under the Single Non-Transferable Vote, The University of Michigan Press 1999, S. 39ff.

${ }^{332}$ Vgl. Blechinger, Verena: Politische Korruption in Japan, a.a.O., S. 332.

${ }^{333}$ Vgl.: Blechinger, Verena: Politische Korruption in Japan, a.a.O., S. 332.

${ }^{334}$ Vater des befragten DPJ-Abgeordneten Matsuno Yorihisa.

${ }^{335}$ Hrebenar, Ronald J.: Rules of the Game: The Impact of the Electoral System on Political Parties, in: Hrebenar, Ronald J: Japan’s new party system, Westview Press, Oxford 2000, S. 43.

${ }^{336}$ Vgl.: Köllner, Patrick: Drei Jahre nach den politischen Reformen - eine Zwischenbilanz. Teil 1: Das Wahlgesetz und seine Auswirkungen, a.a.O., S. 168.

${ }^{337}$ Der 2003 befragte Abgeordnete Matsuno Yorihisa beispielsweise sah sich in der politischen Tradition von Hosokawa, dessen Wahlkreis er übernahm. Auf die Ergebnisse des Interviews wird später genauer eingegangen.

${ }^{338}$ Vgl.: Blechinger, Verena: Politische Reformen in Japan - Auswirkungen und Perspektiven, in: Bosse, Friederike; Köllner Patrick (Hrsg): Reformen in Japan, Mitteilungen des Instituts für Asienkunde Nr. 337, Institut für Asienkunde, Hamburg 2001, S. 91.
} 
Koalitionspartner SPJ Widerstand gegen eine Reform des Wahlsystems, da viele SPJAbgeordnete den Verlust ihrer Mandate, die sie vorher zu einem großen Teil über die hinteren Plätze in den Mehrerwahlkreisen erhalten hatten, fürchteten. Mit überraschenden 13 Stimmen aus der LDP und fünf Gegenstimmen aus der SPJ wurde das Reformpaket am 18.11.1993 im Unterhaus verabschiedet. Die endgültige Verabschiedung des Gesetzes wurde jedoch durch das Oberhaus verzögert. In der Folge kam es zu zahlreichen Verhandlungen zwischen der Koalition und der LDP und anhaltendem Widerstand seitens der SPJ, bis ein Kompromiss gefunden und das Reformpaket am 29.01.1994 in beiden Häusern verabschiedet wurde. Es dauerte jedoch noch ein Jahr, bis die Reformgesetze in Kraft traten. ${ }^{339}$

Das Reformpaket, das 1994 verabschiedet wurde, unterschied sich nicht groß von den Vorschlägen, die schon das Kaifu-Kabinett erarbeitet hatte. Dass die Gesetze am Ende mit den Stimmen der LDP verabschiedet wurden, lag nicht zuletzt daran, dass viele LDP-Abgeordnete fürchteten, für die lange Verzögerung der Reformen von den Wählern bestraft zu werden. ${ }^{340}$

\subsubsection{Die Rolle der Erbabgeordneten im Reformprozess}

Unter den Reformern befanden sich zum einen viele junge Abgeordnete, die mit dem Senioritätssystem der LDP unzufrieden waren Die LDP-Parlamentarier, die 1993 die Fraktion verließen, waren deutlich jünger als die in der Partei verbliebenen Abgeordneten und ihre Wiederwahlraten entsprechend niedrig. Die zehn Abgeordneten, die die Shintô Sakigake gründeten, konnten jeweils nur ein bis drei Wiederwahlen vorweisen: „In other words, the length of parliamentary incumbency correlates inversely with the tendency to defect from the LDP. “341

Zum anderen gab es unter den Politikern, die die LDP verließen, viele jüngere Erbabgeordnete. Axel Klein merkt an, dass diese relativ vermögend waren und ihre Unterstützervereinigungen weitgehend „geerbt“ hatten. Deshalb waren sie nicht so sehr auf die Fortsetzung ihrer politischen Karriere fixiert wie ihre Kollegen und es fiel ihnen daher leichter, politische Reformen zu fordern. ${ }^{342}$

Otake Hideo hat drei Gruppen von Reformern identifiziert, in denen auch Erbabgeordnete eine wichtige Rolle spielten. Neben der bekanntesten Gruppierung, der Utopia Politics Study Group (Yûtopia Seiji Kenkyûkai), gab es die Association to Regain People’s Trust in the LDP und die Young Diet Members for Political Reform. Als Kopf der letztgenannten Gruppe wird Ishiba Shigeru genannt, der im Rahmen dieser Untersuchung im Frühjahr 2005 interviewt wur-

\footnotetext{
${ }^{339}$ Vgl.: Klein, Axel: a.a.O., S. 159ff.

${ }^{340}$ Vgl.: Woodall, Brian: The Politics of Reform in Japan’s Lower House Electoral System, a.a.O., S. 45.

${ }^{341}$ Vgl.: Ebd., S. 43.

${ }^{342}$ Vgl.: Klein, Axel: A.a.O., S. 135.
} 
de. Die Schwerpunkte, die von einer Reform des Wahlsystems bis zu Anti-Korruptionsgesetzen reichten, variierten allerdings je nach Gruppe. ${ }^{343}$

Es erscheint paradox, dass gerade die Abgeordneten, die durch das bestehende politische System offensichtlich Vorteile besaßen, nun für Reformen eintraten. Otake erklärt die Unzufriedenheit vieler junger Erbabgeordneter damit, dass die eigentliche Wahlkampfarbeit selten ihren Vorstellungen von Politik entsprach. In die politischen Debatten konnten sie sich, da sie zumeist erst zwei Jahre im Unterhaus saßen, kaum einbringen. Hinzu kommt, dass viele Abgeordnete regelrecht in diesen Beruf gedrängt wurden. „Discontent was particularly high among the sons of former Diet members (known as nisei politicians), many of whom had been strongly urged by former supporters of their fathers to give up other jobs to take over their father's bases of support. “344

Die große Mehrheit der Abgeordneten, die damals in Parteien wie die Shintô Sakigake wechselten, stammten aus Politikerfamilien. ${ }^{345}$ Tatsächlich fanden sich auch unter den befragten Erbabgeordneten einige Politiker, die während des Reformprozesses und der häufigen Veränderungen im Parteiensystem aus der LDP in andere Parteien wechselten. Allerdings kehrte ein Teil von ihnen bald wieder in die Jimintô zurück.

Hinzu kommt, dass diese Erbabgeordneten aus einer im Vergleich zu anderen Abgeordneten relativ starken Position heraus die LDP-Führung kritisieren konnten, da sie in ihren Wahlkreisen zumeist über sehr starke Unterstützerorganisationen und über große Finanzmittel verfügten, die ihnen eher erlaubten, die LDP zu verlassen. Otake bescheinigt ihnen außerdem eine „Ich kann jederzeit aufhören“-Mentalität. ${ }^{346}$ Das schien vor allem für Erbabgeordnete zu gelten, die erst vor kurzer Zeit ihren ursprünglichen Beruf aufgegeben hatten und darüber hinaus über entsprechende Finanzmittel verfügten.

Das heißt aber nicht, dass es allein junge (Erb-)Abgeordnete waren, die den Reformprozess beschleunigten. Vielmehr bildete sich eine Allianz aus alten und neuen Kräften heraus. Während vor allem die Politiker mittleren Alters auf einen Status Quo bedacht waren (s.o.), befanden sich unter den Reformen auch ältere Politiker der LDP, die kaum noch politische Ambitionen hatten und dadurch seitens der LDP Führung wenig erpressbar waren. Otake spricht daher von einer Allianz junger und alter „Idealisten“. ${ }^{347}$

\footnotetext{
${ }^{343}$ Vgl. Otake, Hideo: Forces for Political Reform: The Liberal Democratic Party’s Young Reformers and Ozawa Ichirô, in: Journal of Japanese Studies, Vol. 22, Nr. 2/1996, S. 273.

${ }^{344}$ Ebd., S. 276.

${ }^{345}$ Vgl.: Ebd., S. 277.

${ }^{346}$ Vgl.: Ebd., S. 277-278.

${ }^{347}$ Vgl.: Ebd., S. 278-280.

Interessant ist, dass Otake an dieser Stelle von einem „feeling of guilt“ spricht, das einige junge Erbabgeordnete aufgrund ihrer Herkunft empfunden hätten und sie bewegt hätte, für Reformen einzutreten.
} 


\subsubsection{Das neue Wahlsystem}

Im Folgenden sollen nun die zentralen Punkte des Reformpakets, die für den Rekrutierungspfad des Erbabgeordneten relevant sind, kurz dargestellt werden.

Mit dem Gesetz zur Wahl in öffentliche Ämter (kôshoku senkyohô) wurde nicht nur ein neues Wahlsystem eingeführt, sondern es wurden auch neue Regelungen für Wahlkreisarbeit und Wahlkampf getroffen. Unter dem neuen Wahlsystem gibt es 480 Unterhauswahlkreise, von denen 300 durch Mehrheitswahl (Erststimme) in Einerwahlkreisen, 180 durch Verhältniswahl über Parteilisten in elf Großwahlkreisen (Zweitstimme), so genannten Blocks, vergeben werden. Die Größe der Blocks liegt zwischen sieben und 21 Mandaten. Die Ergebnisse werden dabei nicht miteinander verrechnet (Parallelsystem oder Grabensystem). ${ }^{348}$

Im Einerwahlkreis gewinnt der Kandidat mit einfacher Mehrheit, sofern seine Stimmenzahl mindestens einem Sechstel der abgegebenen gültigen Stimmen entspricht. Um unseriöse Kandidaturen zu verhindern muss jeder Kandidat eine Kaution von 6 Mio. Yen hinterlegen, die verfällt, wenn er nicht mindestens $10 \%$ der abgegebenen Stimmen erhält. ${ }^{349}$

Für die Kandidaten besteht die Möglichkeit einer Doppelkandidatur, das heißt, dass sie sowohl im Einerwahlkreis als auch auf der Parteiliste kandidieren können. Die Bestimmungen zu den Parteilisten sind nicht ganz unkompliziert und weisen einige Besonderheiten auf. Eine Partei kann mehrere Kandidaten auf den gleichen Listenplatz setzen. Zunächst werden die Namen der Kandidaten von der Liste gestrichen, die ein Direktmandat gewonnen haben. Die restlichen Plätze werden gemäß der Liste gegeben. Stehen mehrere Kandidaten auf dem selben Listenplatz, der Partei steht aber z.B. nur noch ein Mandat zu, zieht derjenige Kandidat ins Parlament ein, der am besten in seinem Wahlkreis abgeschnitten hat. Das Ergebnis entscheidet sich nach der erhaltenen Stimmenzahl im Verhältnis zum Sieger des Wahlkreises. ${ }^{350}$

Eine Parteiliste kann eine Partei jedoch nur aufstellen, wenn sie eines der folgenden Kriterien erfüllt: 1. Die Partei hat bei der letzten Wahl (Ober- oder Unterhaus) mindestens 2\% der Stimmen erhalten. 2. Die Partei verfügt über mindestens fünf Abgeordnete im Ober- oder Unterhaus (oder in beiden zusammen). Eine Partei, die eine dieser Vorraussetzungen erfüllt, muss dann eine Mindestanzahl von Kandidaten aufstellen, die 20\% der zu vergebenen Mandate entspricht. ${ }^{351}$ Es gilt eine Sperrklausel von 3\%. ${ }^{352}$

\footnotetext{
${ }^{348}$ Vgl.: Bruns, Gabriele: A.a.O., S. 223-227.

${ }^{349}$ Vgl.: Klein, Axel: A.a.O., S. 181f.

${ }^{350}$ Vgl.: Köllner, Patrick: Drei Jahre nach den politischen Reformen - Eine Zwischenbilanz. Teil I: Das Wahlgesetz und seine Auswirkungen, a.a.O., S. 168f.; Christensen, Raymond V.: The New Japanese Election System, in: Pacific Affairs, No. 69/1996, S. 54.

${ }^{351}$ Vgl.: Christensen, Raymond V.: The new Japanese election system, a.a.O., S. 53f.; Klein, Axel: A.a.O., S. 181.

${ }^{352}$ Vgl.: Bruns, Gabriele: A.a.O., S. 225; Klein, Axel: A.a.O., S. 181.
} 
Nach wie vor müssen die Wähler den Namen des Kandidaten selbst auf dem Wahlzettel eintragen, was von der LDP damit begründet wurde, dass zu groß geratene Wahlzettel die Stimmenauszählung erschwert hätten. Kurzzeitig war zwar angedacht worden, die Namen der Kandidaten auf die Stimmzettel zu drucken. Diese Änderung wurde aber 1995 von einer Koalition aus LDP, SDPJ und Shintô Sakigake wieder rückgängig gemacht. ${ }^{353}$

Die sehr strengen Regelungen, die für Wahlkämpfe und Wahlkampfaktivitäten galten, wurden hingegen kaum gelockert: „In particular, door-to-door campaigning, signature drives, polling, providing food and drink, mass meetings, parades, unscheduled speeches, multiple campaign vehicles, and candidate-produced literature are illegal in contemporary Japanese campaigns. Instead, a candidate's contacts with his or her potential voters must, by law, be channeled through a limited number of government-produced postcards, posters, placed on official signboards, a maximum of five government paid newspaper ads of a specific size and content, several television radio announcements, a number of joint speech meetings, and government financed handbills and brochures. ${ }^{354}$ Die Vorschriften und Restriktionen für Wahlkampfaktivitäten umfassen etwa 480 Seiten.

Ein Fortschritt in Richtung Korruptionsbekämpfung ist die Einführung der Mithaftung (renzasei) bei Vergehen gegen diese Regelungen. Macht sich jemand aus dem engeren Umfeld des Kandidaten, z.B. ein Familienangehöriger, Sekretär oder Mitarbeiter, illegaler Aktivitäten schuldig, kann der Kandidat haftbar gemacht werden, auch wenn dieser Verstoß gegen das Wahlkampfgesetz ohne sein Wissen geschehen ist. Die Strafmaße bei Verstößen gegen das Wahlgesetz wurden ebenfalls beträchtlich erhöht. Auf diesem Gebiet scheint die Korruptionsbekämpfung, wie eine Reihe von Ermittlungsverfahren zeigt, relativ erfolgreich zu sein. ${ }^{355}$

Mit dem Gesetz bezüglich der Einrichtung einer Wahlkreiskommission wurde eine Neueinteilung der Wahlkreise beschlossen, nicht zuletzt um die Ungleichheit des Stimmenwertes unter dem 1955er-System zu beseitigen. Diese Kommission soll auch in Zukunft nach jedem Zensus die Wahlkreiseinteilung verändern können. Als Ziel der Neueinteilung war formuliert worden, die Stimmenungleichheit unter 1:2 zu halten. Dieses Ziel wurde allerdings nicht ganz erreicht, es existieren einige Wahlkreise mit Stimmenungleichheit. Christensen urteilt aber über die neuen Wahlkreise, dass hier im Gegensatz zu den USA ein gerrymandering weitgehend vermieden werden konnte. ${ }^{356}$

\footnotetext{
${ }^{353}$ Vgl.: Köllner, Patrick: Drei Jahre nach den politischen Reformen - Eine Zwischenbilanz. Teil I: Das Wahlgesetz und seine Auswirkungen, a.a.O., S. 168.

${ }^{354}$ Hrebenar, Ronald J.: Rules of the Game: The Impact of the Electoral System on Political Parties, a.a.O., S. 50f.

355 Blechinger, Verena: Politische Reformen in Japan - Auswirkungen und Perspektiven, a.a.O., S. 101.

${ }^{356}$ Vgl.: Christensen, Raymond V.: The new Japanese election system, a.a.O., S. 55f.
} 


\subsubsection{Die Neuregelung der Politikfinanzierung}

Mit dem Gesetz zur Regulierung politischer Geldzuwendungen (seiji shikin kiseihô) und dem Gesetz über die Subventionierung von Parteien (seitô joseihô) sollten Probleme wie die hohen Kosten der Wahlkämpfe und die mangelnde Transparenz bei der politischen Finanzierung gelöst werden. ${ }^{357}$

Spenden von Unternehmen oder Verbänden an Einzelpolitiker wurden nach einer Übergangszeit, in der ein Unternehmen bis zu 500.000 Yen an offizielle Finanzverwaltungsorganisationen von Politikern spenden durfte, ab dem Jahr 2000 ganz verboten. Die Einkommensverluste sollen durch die Förderung von Individualspenden, mit Hilfe von Steuerbegünstigungen und durch eine staatliche Parteienfinanzierung ausgeglichen werden. Man hoffte so, durch die Zahlung der staatlichen Mittel an die Zentralebenen der Partei diese in „ihrer Funktion als Instrument der politischen Interessenaggregation und -vermittlung “ ${ }^{358} \mathrm{zu}$ stärken und die habatsu als Geldumverteilungsmaschinen überflüssig zu machen.

Unternehmen und Verbände dürfen nun, je nach Größe des Unternehmens, Spenden zwischen 7,5 und 100 Mio. Yen im Jahr an politische Parteien richten. Allerdings gibt es hier eine gravierende Gesetzeslücke, wie Verena Blechinger bemerkt. Es existieren keine Beschränkungen für Spenden an lokale oder regionale Organisationen der Parteien und auch keine Höchstgrenze für lokale Parteiorganisationen in einem Wahlkreis. Viele Einzelpolitiker, die laut Gesetz nun keine Unternehmensspenden mehr erhalten dürfen, wandelten ihre kôenkai deshalb in lokale Parteiorganisationen um, deren Zahl in der LDP seit 1994 erheblich angestiegen ist. ${ }^{359}$

Natürliche Personen können bis zu 20 Mio. Yen im Jahr an politische Organisationen spenden, weitere 10 Mio. Yen an sonstige Adressaten, wie etwa persönliche Unterstützervereinigungen. Um Individualspenden zu fördern, können diese steuerlich geltend gemacht werden. ${ }^{360}$

Für mehr Transparenz bei der Spendenpraxis soll die namentliche Offenlegung von Spenden dienen. Die Mindestbeträge hierfür wurden auf 50.000 Yen gesenkt. Sehr wichtig ist in diesem Zusammenhang die Bestimmung zu Fundraising-Parties, die für viele Politiker (und auch Parteien und habatsu) nach wie vor eine wichtige Einnahmequelle darstellen. Die Namen von Käufern, die Eintrittskarten im Wert von mehr als 500.000 Yen kaufen, müssen nun offen gelegt werden. ${ }^{361}$

\footnotetext{
${ }^{357}$ Vgl.: Blechinger, Verena: Auf dem Weg zu „sauberer Politik“ und transparenten Strukturen? A.a.O., S. $164 f$.

${ }^{358}$ Köllner, Patrick: Drei Jahre nach den politischen Reformen - Eine Zwischenbilanz Teil 2: Die Finanzierung politischer Aktivitäten, in: Japan aktuell. Wirtschaft. Politik. Gesellschaft, Heft Juni/1998, S. $274 f$.

${ }^{359}$ Vgl.: Blechinger, Verena: Politische Reformen in Japan - Auswirkungen und Perspektiven, a.a.O., S.

${ }^{360}$ Vgl.: Christensen, Raymond V.: The new Japanese election system, a.a.O., S. 57.

${ }^{361}$ Vgl.: Köllner, Patrick: Parteienfinanzierung in Japan: Regulierungsmechanismen und Anpassungsstrategien, in: Japan aktuell. Wirtschaft. Politik. Gesellschaft, Heft April/2000, S. 150.
} 
Um die erwarteten Spendenausfälle zu kompensieren, wurde eine öffentliche Parteienfinanzierung eingeführt. Sie umfasst 250 Yen pro japanischem Staatsbürger, also etwa 31 Mrd. Yen, und sollte den erwarteten Finanzbedarf (1994) der Parteien zu einem Drittel decken. Vermutlich liegt der tatsächliche Finanzbedarf der Parteien aber wesentlich höher. Anspruchsberechtigt sind Parteien, die bei der letzten Wahl auf nationaler Ebene mindestens 2\% der Stimmen bekommen haben oder über fünf Abgeordnete in Ober- oder Unterhaus verfügen. Über die Verwendung dieser Mittel müssen Rechenschaftsberichte verfasst werden. ${ }^{362}$

\section{Abbildung 13: Die neuen Bestimmungen zur Politikfinanzierung}

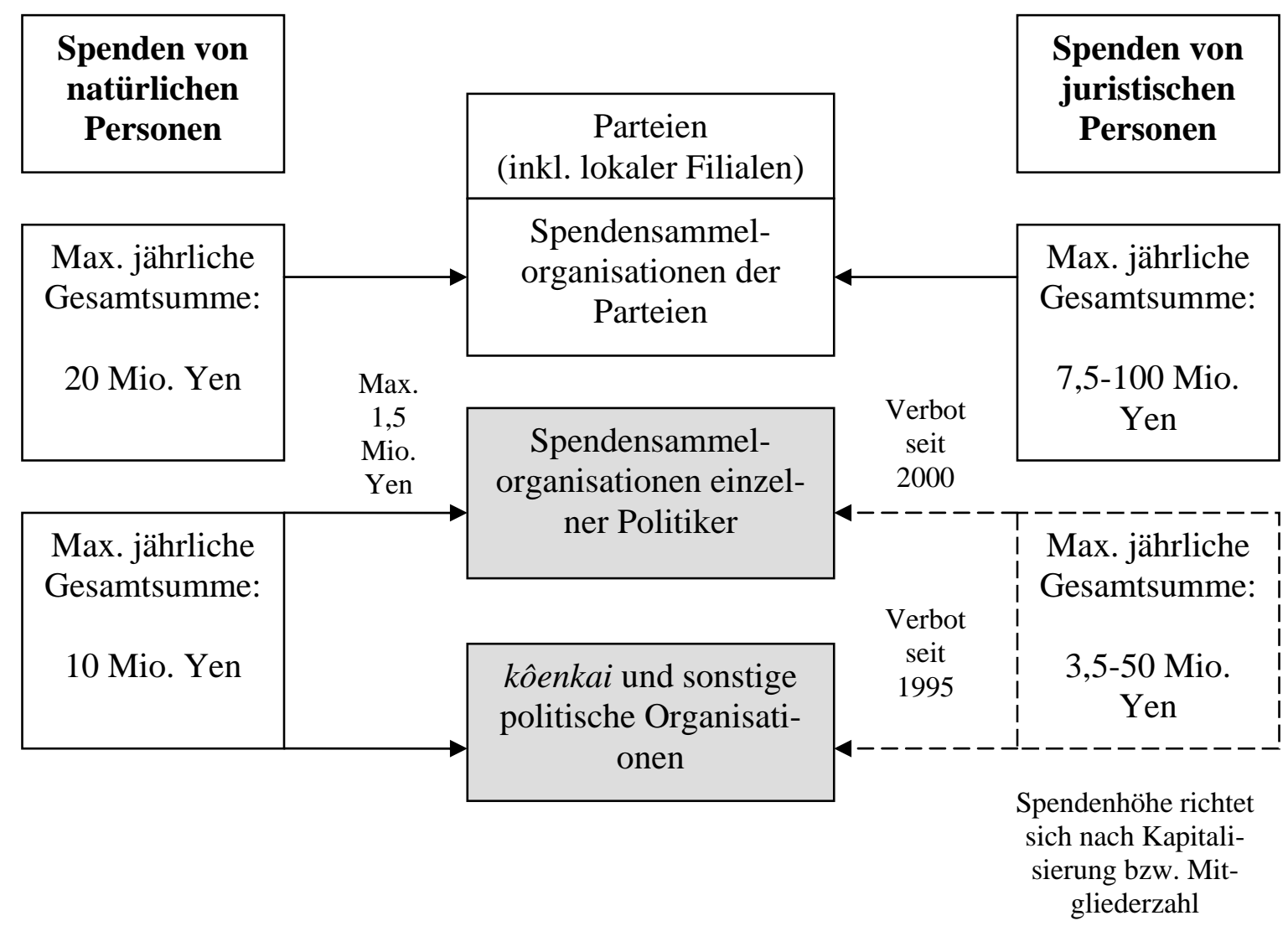

Nach: Köllner, Patrick: Parteienfinanzierung in Japan: Regulierungsmechanismen und Anpassungsstrategien, in: Japan aktuell. Wirtschaft. Politik. Gesellschaft, Institut für Asienkunde Hamburg 2000, Heft 2/2000, S. 151. 


\subsection{Die Auswirkungen der politischen Reformen}

Die Erwartungen, die an die politischen Reformen geknüpft wurden, waren sehr hoch: „It is an important reform, that will affect the number and type of political parties as well as the type and activities of Diet candidates.” ${ }^{363}$ Eine ganze Reihe von Hypothesen über die Folgen der Reformen wurden aufgestellt, von denen sich einige auch explizit auf die Kandidatenrekrutierung bezogen, wie etwa das Voranschreiten des Generationswechsels in den Parteien und die Erhöhung des Anteils weiblicher Abgeordneter. ${ }^{364}$

Auch indirekt betreffen die Reformen die politische Rekrutierung, etwa durch eine veränderte Opportunitätsstruktur bzw. Demand-side. An dieser Stelle wäre etwa die erhoffte Begrenzung der kostenintensiven und stark personalisierten Wahlkämpfe und die dadurch prophezeite Stärkung der offiziellen Parteiorganisationen auf Kosten der habatsu zu nennen. Interessant ist die Annahme, dass sich die Abgeordneten auf Listenplätzen eher auf nationale Fragen konzentrieren würden, wohingegen die Beziehungen zwischen Abgeordneten in den Einerwahlkreisen und ihren Wählern enger würden. ${ }^{365}$

Natürlich wurde auch mit Kritik nicht gespart. Einige dieser Kritikpunkte werden noch im Rahmen der Interviewanalysen angesprochen, aber man kann vorwegnehmen, dass die Reformen bei den Abgeordneten selbst für wenig Begeisterung sorgten. Wissenschaftler wie Shiratori Rei hatten schon kurz nach der Verabschiedung des Reformpakets große Zweifel, ob die Reformen ein Erfolg werden würden. ${ }^{366}$

Im Folgenden soll nun geklärt werden, welche Auswirkungen die politischen Reformen auf die Kandidatenrekrutierung in Japan gehabt haben, welche Veränderungen man auf der Nachfrageseite feststellen kann und in welchem Umfang die Angebotsseite von dem Wandel des politischen Systems betroffen ist.

\subsubsection{Wahlkämpfe unter dem neuen Wahlsystem}

\subsubsection{Die Themen}

Ein wichtiges Ziel der politischen Reformen war, die personenorientierten Wahlkämpfe durch themenorientierte Wahlkämpfe zu ersetzen, um so die Wahlkampfkosten zu senken und inhaltliche Auseinandersetzungen zwischen den Kandidaten zu fördern. Tatsächlich ist es aber

\footnotetext{
${ }^{363}$ Christensen, Raymond V.: The New Japanese Election System, a.a.O., S. 70

${ }^{364}$ Vgl.: Köllner, Patrick: Drei Jahre nach den politischen Reformen - Eine Zwischenbilanz, Teil 1: Das Wahlgesetz und seine Auswirkungen, a.a.O., S. 169.

${ }^{365}$ Vgl.: Ebd, S. 169.

${ }^{366}$ Vgl.: Shiratori, Rei: Shiratori, Rei: Shôsenkyokusei-de Seiji-ha dô naru-ka, Libati-Shobô, Tôkyô 1995, S. 74-

78. (Shiratori Rei: Wie entwickelt sich die Politik unter dem Einerwahlsystem?)
} 
fraglich, ob dieses Ziel erreicht wurde. Denn die Wahlkampfführung scheint sich auch zehn Jahre nach Inkrafttreten der Reformgesetze kaum geändert zu haben. ${ }^{367}$ Viele Interviewpartner waren der Meinung, dass es keinen grundlegenden Wandel der Wahlkämpfe und des Wahlkampfstils gegeben hat.

Wahlkämpfe sind also in den Wahlkreisen selbst noch sehr personenzentriert. Ausgerichtet auf die Person des Kandidaten zählen in den Wahlkämpfen vor allem die „zwischenmenschlichen Beziehungen“ (jinmyaku). ${ }^{368}$ Regionale Themen sind von großer Bedeutung, parteipolitische Auseinandersetzungen eher selten. ${ }^{369}$ Ein Teil der befragten Abgeordneten war allerdings anderer Meinung, sowohl die Politiker von der DPJ als auch drei Abgeordnete von der LDP (Harada, Ishiba, Kosaka). Harada Yoshitsugu: „[...] Was sich geändert hat, ist, dass die Zeit des Regionalismus vorbei ist und die politischen Themen nicht mehr lokal sind, sondern globale Probleme, auf einer nationalen Ebene, sind. Also Umweltprobleme oder so genannte ,new issues'. Das heißt, dass die Abgeordneten nicht mehr nur wegen ihrer Verdienste vor Ort gewählt werden. Diese Veränderung hat es gegeben. Jetzt ist bei der Auswahl auch diese politische Strategie, die ,policy’, wichtig. “(Harada)

Der Abgeordnete Ishiba stand der Reform, nicht nur wegen seiner Rolle während des Reformprozesses (s.o.), positiv gegenüber. Da er nicht mehr gegen andere Kandidaten seiner Partei kämpft und daher keine Rücksicht mehr auf die Faktionszugehörigkeit zu nehmen braucht, sei es für ihn einfacher, in seinem Wahlkampf mit nationalen Themen, z.B. für Koizumi als Ministerpräsident, zu werben: „,Bei der Wahl sage ich, dass der nächste Ministerpräsident Koizumi heißen sollte. Mir eine Stimme zu geben, heißt, Herrn Koizumi eine Stimme zu geben. [...] Das heißt, man kann einen Wahlkampf führen, bei dem alle in der LDP an einem Strang ziehen. Man kann sagen: ,Bitte wählen Sie alle mich, um den Vorsitzenden, den Chef der LDP, Herrn Koizumi zum Ministerpräsidenten zu machen.’ Das finde ich, ist ein gutes Wahlsystem. “

Tatsächlich scheint sich die Rolle des Ministerpräsidenten im Wahlkampf grundsätzlich verändert zu haben. Krauss und Nyblade beobachten eine zunehmende Bedeutung der Person des Premierministers über den Zeitraum der letzten 20 Jahre. Eine verstärkte Ausrichtung des Wahlkampfes auf die Person des Regierungschefs macht in der LDP schon deshalb Sinn, weil dessen Popularität in den letzten Jahren konstant höher lag als die seiner Partei, wobei Koizumi Junichirô derzeit sicherlich einen Extremfall darstellt. ${ }^{370}$ Unter dem Gesichtspunkt der zuneh-

\footnotetext{
${ }^{367}$ Vgl.: Christensen, Raymond V.: The Effect of electoral Reform on Campaign Practices in Japan. Putting New Wine into Old Bottles, in: Asian Survey, No. 38/1998, S. 1003

${ }^{368}$ Vgl.: Park, Cheol-Hee: A.a.O., S. 63.

${ }^{369}$ Vgl.: Bruns, Gabriele: A.a.O., S. 263ff.

${ }^{370}$ Vgl.. Krauss, Ellis S.; Nyblade, Benjamin: “Presidentialization” in Japan? The Prime Minister, Media and Elections in Japan, in: British Journal of Political Studies, Vol. 35, No. 2/2005, S. 360ff.
} 
menden Bedeutung der Attraktivität des Ministerpräsidenten bzw. des Spitzenkandidaten ist also die Antwort des Abgeordneten Ishiba leicht nachvollziehbar. Krauss und Nyblade kommen zu dem Schluss: „While the Japanese prime minister may not necessarily be the strong leader seen in many other countries, most notably Britain, the public image of the prime minister has become a more potent force in Japanese politics. “371

Ansonsten herrschte die Meinung vor, die Wahlkämpfe hätten sich kaum verändert. Ein anderes Problem ergibt sich aus der Tatsache, dass Kandidaten jetzt die Mehrheit der Stimmen in ihrem Wahlkreis bekommen müssen. Weil die Kandidaten also eine möglichst große Zahl von Wählern für sich gewinnen wollen und nicht mehr für eine relativ kleine Klientel Politik machen, wirken die Programme häufig konturlos. Kandidaten sprechen eher lokale und regionale Themen an, die als unverfänglich gelten. Parteipolitische Themen sind eher selten. Klein nennt sogar Fälle, in denen Abgeordneten im Wahlkampf auf die Nennung ihrer Partei ganz verzichteten. ${ }^{372}$ Dieses Problem wurde auch von den befragten Abgeordneten angesprochen: „Was sich in letzter Zeit allerdings geändert hat und was den Abgeordneten Probleme bereitet, ist, dass sie zu jedem ein ,freundliches Gesicht' machen müssen. Das heißt zum Beispiel, dass man sowohl zu den Befürwortern als auch zu den Kritikern von Atomkraft sagen muss: ,Ok, Ok, was sie sagen ist richtig.' Aber schließlich gibt man keine eigenen Argumente ab. “ (Watanabe)

Es lässt sich also festhalten, dass sich die Hoffnungen auf mehr programmatische Auseinandersetzungen und weniger personenzentrierte Wahlkämpfe wohl nur in Teilen erfüllt hat. Lediglich 40\% der Kandidaten erklärten in der Wahl 2003, voll hinter dem Wahlprogramm ihrer Partei zu stehen. In der LDP waren es sogar nur $12 \%{ }^{373}$

\subsubsection{Der Wahlkampfstil}

Nach Park bestehen japanische Wahlkämpfe immer noch in erster Linie aus aisatsu (Begrüßen), akushû (Händeschütteln) und kaiwa (Small Talk). Der Wahlkampf des Kandidaten beschränke sich eigentlich nur darauf, den Wähler zu bitten, ihn zu wählen. ${ }^{374}$

Wie bereits erwähnt, sind die japanischen Wahlkämpfe immer noch sehr stark auf die Person des einzelnen Kandidaten ausgerichtet. Klein nennt als Wahlkampfmittel vor allem das permanente Ausrufen des Namens (renko) und so genannten „Überrollstrategien“. Hierbei „überrollen“ die Wahlkampfhelfer sprichwörtlich ganze Wohnviertel, um an der Haustür den Wähler direkt anzusprechen und vor allem den Namen des Politikers bekannt zu machen. Diese Strategie wird auch dobuita senkyo genannt, weil der Kandidat dabei die Abdeckung der Abwasser-

\footnotetext{
${ }^{371}$ Ebd., S. 368.

${ }^{372}$ Vgl.: Klein, Axel: A.a.O., S. 228ff.

${ }^{373}$ Vgl.: Japan aktuell: Die Unterhauswahl vom November 2003 im Überblick, in: Japan aktuell. Wirtschaft. Politik. Gesellschaft, Heft Dezember/2003, S. 530.

${ }^{374}$ Vgl.: Park, Cheol-Hee: A.a.O., S. 81.
} 
rinnen am Straßenrand (dobuita) überqueren muss. Im Gegensatz zu dieser Taktik, die eigentlich gesetzlich verboten ist, unterliegen Telefonanrufe keiner Beschränkung, so dass hiervon viel Gebrauch gemacht wird. In diesem Sinne scheint sich somit an den Wahlkampfmitteln und -stilen selbst nichts oder wenig geändert zu haben. ${ }^{375}$

Ähnlicher Meinung waren auch die befragten Erbabgeordneten. Fast alle Interviewpartner betonten die Bedeutung lokaler Aktivitäten, auch außerhalb des Wahlkampfes. „[...] Was die Aktivitäten in den Wahlkreisen betrifft, werden immer noch viele Partys und Versammlungen durchgeführt, wie etwa Buffets, Partys, Ausflüge, außerdem ,Town Meetings’. Das hat sich kaum geändert.“ (Watanabe)

Interessanterweise scheint der Einsatz (neuer) Medien im Wahlkampf gemessen an anderen Ländern noch recht gering ausgeprägt zu sein, auch wenn er auf zentraler Ebene tendenziell zugenommen hat. Terata Manabu, jüngster Abgeordneter unter den Interviewpartnern, betonte die Bedeutung moderner Informationstechnologie: „Unter dem 1955er-System gab es fast noch keine Informationstechnologie. Der Rundfunk war noch sehr schwach entwickelt. Jetzt gibt es ja das Internet, das Fernsehen bringt sehr viele Informationen, die Massenmedien, alle Arten von Zeitungen berichten... Das, was die Kandidaten denken, kann einfacher verbreitet werden. Das hat sich verglichen mit dem 1955er-System ausgeweitet. Daher ist das viele Händeschütteln nicht mehr der Maßstab der Motivation bei den Wählern. “ Nach Christensen haben die Verantwortlichen für die Wahlkämpfe die Wirksamkeit von „Medienwahlkämpfen“ vor allem in den Städten und bei der Gewinnung von Wechselwählern erkannt, setzen aber dennoch in erster Linie auf herkömmliche Strategien. ${ }^{376}$

Ein Grund hierfür könnten die strengen Wahlkampfbestimmungen sein. Die relativ restriktiven Wahlkampfgesetze in Japan sind seit je her dem Vorwurf ausgesetzt, eher kontraproduktiv zu wirken. So darf im Internet nicht mit dem Namen des Kandidaten geworben werden. Auch Haustürbesuche sind verboten, Anzeigen und Werbung so stark reglementiert, dass „informelle Kontakte“ und Aktivitäten in der Vorwahlkampfzeit die beste Möglichkeit darstellen, mit den Wählern in Kontakt zu kommen. „Even if candidates wanted to persue different campaign strategies, they are prohibited by strict campaign laws from enhancing the direct appeal or advertising components of their campaigns. With these restrictions in place, candidates are essentially prohibited from running issue-oriented campaigns. " 377

Eine gute Möglichkeit, mit dem Wähler in Kontakt zu kommen, stellen deshalb nach wie vor die kôenkai dar, die immer noch als wichtigstes Wahlkampfmittel gelten. Da bis auf die KPJ

\footnotetext{
${ }^{375}$ Vgl.: Klein, Axel: A.a.O., S. 227ff.

${ }^{376}$ Vgl.: Christensen, Raymond V.: The Effect of electoral Reforms on Campaign Practices in Japan, a.a.O., S. 1004

${ }^{377}$ Christensen, Raymond V.: The New Japanese Election System, a.a.O., S. 67.
} 
die lokalen Parteiorganisationen häufig noch zu schwach sind, setzen vor allem die Kandidaten konservativer Parteien in den Wahlen auf Netzwerke, die sich um ihre kôenkai herum gruppieren. Dabei werden die Vorsitzenden bestimmter Verbände, z.B. Ärztevereinigungen, Landwirtschaftsverbände, Vereinigungen der örtlichen Händler etc., zu Funktionsträgern (sewanin) in den kôenkai gemacht. Im Wahlkampf kann dann auf eine ganze Reihe Organisationen zurückgegriffen werden. ${ }^{378}$

Köllner merkt dagegen an, dass an vielen Stellen schon funktionstüchtige Ortsgruppen geschaffen wurden, die finanziell hinreichend ausgestattet sind, um selbst Aktivitäten vor Ort zu organisieren. Diese spielen zunehmend eine wichtige Rolle. Allerdings sind die Vorsitzenden dieser Gruppen oft gleichzeitig der örtliche LDP-Kandidat (z.B. der befragte Politiker Usui Hideo in Chiba). So nutzen viele Kandidaten die örtlichen Parteiorganisationen als ihre kôenkai oder verschmelzen diese Gruppen miteinander. ${ }^{379}$ Viele Unterstützerorganisationen wurden auch deshalb in offizielle Parteiorganisationen umgewandelt, um weiterhin Unternehmensspenden empfangen zu können. Diese Zuwendungen gehen dann offiziell an die Ortsgruppe der Partei, landen tatsächlich aber wieder beim einzelnen Kandidaten. Als die LDP Anfang der 1990er Jahre die Neugründung von lokalen Parteiorganisationen forcierte, schossen in der Folgezeit neue Ortsgruppen aus dem Boden wie „Bambus nach dem Regen““ ${ }^{380}$ Nachdem die LDP ihren Abgeordneten erlaubt hatte, jeweils eigene Parteiorganisationen zu gründen („Verfeinerung der Parteigliederung“), stieg die Zahl der Lokalorganisationen von 5100 (1990) auf 5700 (1999) an. ${ }^{381}$

Auf die Bedeutung der kôenkai für die Kandidaten im Zusammenhang mit der Erbfolge wird später noch genauer eingegangen. Man kann aber festhalten, dass die persönlichen Unterstützerorganisationen zumindest in der LDP nach wie vor eine wichtige Rolle für die Stimmenmobilisierung spielen und in vielen Fällen wohl keine Alternative zu ihnen besteht. Hierbei kann es allerdings erhebliche Unterschiede zwischen Stadt und Land geben.

Krauss und Pekkanen bezeichnen die kôenkai sogar als Lösung und Problem der LDPKandidaten zugleich. Diese müssen sich angesichts einer geringen Parteiidentifikation verstärkt um Wähler bemühen, die sich zwar nicht mit der LDP, wohl aber mit dem Kandidaten identifizieren und daher seiner Unterstützerorganisation beitreten. Die Mitgliedschaft in einer kôenkai

\footnotetext{
${ }^{378}$ Vgl.: Park, Cheol-Hee: A.a.O., S. 80f.

${ }^{379}$ Vgl.: Köllner, Patrick: Drei Jahre nach den politischen Reformen - Eine Zwischenbilanz Teil 1: Das Wahlgesetz und seine Auswirkungen, a.a.O., S. 174.

${ }^{380}$ Vgl.: Krauss, Ellis S.; Pekkanen, Robert: A.a.O., S. 10; Klein, Axel: A.a.O., S. 249.

${ }^{381}$ Vgl.: Klein, Axel: A.a.O, S. 276; Kagaya, Katsuo: A.a.O., S. 104-105., Köllner, Patrick: Parteienfinanzierung in Japan, a.a.O., S. $154 \mathrm{ff}$.
} 
führt jedoch umgekehrt zu einer geringeren Identifikation mit der LDP als es die Mitgliedschaft in einer Ortsgruppe tun würde. ${ }^{382}$

\subsubsection{Die Stellung des Abgeordneten in seinem Wahlkreis}

Eine Veränderung, die mit der Einführung des neuen Wahlsystems eintrat, ist die Stellung des Abgeordneten in seinem Wahlkreis. Die meisten befragten Erbabgeordneten waren der Meinung, dass der einzelne Abgeordnete an Macht gewonnen habe, da nur noch ein Mandat pro Wahlkreis vergeben wird. „Das war bisher das Gute an den Mehrerwahlkreisen, dass es in einem Wahlkreis viele Abgeordnete gab. Daher hielten sich die verschiedenen Leute notwendigerweise gegenseitig unter Kontrolle, hielten sich gegenseitig davon ab, Vorrechte anzustreben und so weiter. Es ist doch so, dass es einfacher ist, die Macht zu konzentrieren, weil es jetzt in einem Wahlkreis nur noch einen Abgeordneten gibt, nicht wahr? (Hamada)

Durch die herausragende Stellung des Abgeordneten im Wahlkreis wird der Abgeordnete zu einer Art „kleiner Feudalherr“, wie Köllner anmerkt. ${ }^{383}$ In diesem Sinn, und darin stimmten viele der befragten Politiker überein, wird die Tendenz zu einer Politik, die sich nur auf den Wahlkreis konzentriert, eher verstärkt. Nach Klein „warnten“ einige LDP-Abgeordnete die Wähler sogar davor, sich „falsch“ zu entscheiden, da sie nun die einzigen seien, die über ihre guten Kontakte auf nationaler Ebene Wohltaten in den Wahlkreis leiten könnten. ${ }^{384}$

Für einen Herausforderer ist deshalb außerordentlich schwer, gegen den Amtsinhaber zu gewinnen. Bruns weist darauf hin, dass in Systemen mit Einerwahlkreisen die Amtsinhaber tendenziell bevorzugt werden. Sie belegt dies damit, dass 1996 nur 11\% der Erstkandidaten gegen einen Amtsinhaber gewinnen konnten. Vor allem die mit einem Regierungsbonus ausgestatteten Amtsinhaber der LDP könnten in vielen Fällen die Ressourcen der Partei bündeln und einsetzen. $^{385}$

Die Mindeststimmenanzahl für einen Mandatsgewinn schwanken sehr stark. Wo mehrere starke Kandidaten gegeneinander antreten, reichen mitunter schon 25\% der abgegebenen Stimmen, um ein Mandat zu erringen. In Wahlkreisen mit nur zwei relevanten Kandidaten dagegen muss ein Kandidat etwa 45\% der Stimmen für sich gewinnen. In beiden Fällen ist die Zahl der „toten“ Stimmen relativ hoch. Dies ist ebenfalls ein Kritikpunkt, den einige Interviewpartner an den politischen Reformen äußerten. Gerade Minderheitenmeinungen könnten nicht mehr repräsentiert werden. Allerdings gibt es auch Wahlkreise, in denen die Mehrheits-

\footnotetext{
${ }^{382}$ Vgl.: Krauss, Ellis S.; Pekannen, Robert: A.a.O., S. 12.

${ }^{383}$ Vgl.: Köllner, Patrick: : Drei Jahre nach den politischen Reformen - Eine Zwischenbilanz Teil 1: Das Wahlgesetz und seine Auswirkungen, a.a.O., S. 170.

${ }^{384}$ Vgl.: Klein, Axel: A.a.O., S. 229.

${ }^{385}$ Vgl.: Bruns, Gabriele: A.a.O., S. 273f.

Hierauf wird im Zusammenhang mit den Ressourcen der Erbabgeordneten noch genauer eingegangen.
} 
verhältnisse relativ eindeutig sind und der Wahlsieger über 70\% der Stimmen erhält. ${ }^{386}$ Dennoch sollte man bei diesen Zahlen nicht vergessen, dass die Wahlbeteiligung in Japan derzeit relativ niedrig ist und bei etwa 59,9\% liegt. ${ }^{387}$

In einigen Fällen, vor allem in ländlichen Gebieten, wurde die Neueinteilung der Wahlkreise zum Vorteil der Mandatsträger vorgenommen. ${ }^{388}$ Ein Beispiel hierfür ist der Abgeordnete Hori, der in seinem Wahlkreis etwa 103.000 Stimmen erhielt, seine DPJ-Herausforderin jedoch nur 36.000 Stimmen. Seine Wiederwahl, so der Abgeordnete, sei noch nie in Gefahr gewesen. Hori sieht darin allerdings ein demokratietheoretisches Problem.: „In meinem Wahlkreis ist die LDP ja glücklicherweise sehr stark. Ich bekam etwa 100.000 Stimmen, die DPJ, eine Kandidatin, nur 30.000 Stimmen. Es geht ja aber nicht, dass andere Kandidaten gar nicht mehr antreten. Sie werden sich nie mit mir messen können, aber es geht nicht, dass ich ohne Wahl ein Mandat bekomme, weil niemand anders antritt. Deshalb ermuntere ich die Leute von der DPJ anzutreten und zu kämpfen.“

Diese Situation führte Hori vor allem auf die Neuordnung seines Wahlkreises zurück. Er gab allerdings zu bedenken, dass der Wettbewerb in den Städten zugenommen habe und es dort für LDP-Kandidaten ungleich schwerer sei.

\subsubsection{Wahlkampfkosten und Korruptionsvorfälle}

Zu den Wahlkampfkosten nach der Einführung des neuen Wahlsystems gibt es mitunter widersprüchliche Einschätzungen. An der Hypothese, das neue Wahlsystem könne auch die Wahlkampfkosten senken, gab es von Anfang an Kritik. Shiratori Rei nannte den Fall Korea als Beispiel dafür, dass die Einführung von Einerwahlkreisen nicht zwangsläufig zu einer Begrenzung der Ausgaben führt. Korea stellte ebenfalls seine Mehrerwahlkreise, in denen jeweils zwei Abgeordnete gewählt wurden, auf Einerwahlkreise um. Die Erfahrungen aus Korea würden zeigen, so Shiratori, dass die Kandidaten, die ja nun die Mehrheit in ihrem Wahlkreis erringen müssen, ihre Aktivitäten ausweiten und die Auseinandersetzungen an Schärfe zunehmen. ${ }^{389}$ Auch Park merkt an, dass der Übergang vom „Minimalprinzip“, also die Konzentration auf eine kleine aber feste Basis, zum „Maximalprinzip“ unter dem neuen Wahlsystem einen höheren (finanziellen) Aufwand bedeutet. ${ }^{390}$

Dennoch sind die Ausgaben der meisten Kandidaten in den letzten Wahlen leicht gesunken. Tendenziell spricht Köllner von einer Verringerung der Wahlkampfkosten bei den einzelnen Kandidaten und einer Erhöhung der Ausgaben auf der zentralen Ebene durch einen verstärkten

\footnotetext{
${ }^{386}$ Vgl.: Klein, Axel: A.a.O., S. $222 f$.

${ }^{387}$ Vgl.: Japan aktuell: Die Unterhauswahl vom November 2003 im Überblick, a.a.O., S. 530.

${ }^{388}$ Vgl.: Bruns, Gabriele: A.a.O., S. 256.

${ }^{389}$ Vgl.: Shiratori, Rei: A.a.O., S. 75.

${ }^{390}$ Vgl.: Park, Cheol-Hee: A.a.O., S. 67,
} 
Wahlkampf in den Medien. ${ }^{391}$ So äußerte sich auch der Abgeordnete Kosaka im Interview: „Wenn es einen Wahlkampf [unter dem alten Wahlsystem - d.A.] gab, konnte man ins Büro kommen und dort essen. Viel essen, nicht wahr, trinken und dann nach Hause gehen. [...] Als Ergebnis der politischen Reformen brauche ich nicht mehr so viel Geld in meinem Wahlkreis. [...] Man denkt über einen Weg nach, möglichst wenig Geld zu benutzen. Ein Resultat ist also, dass man weniger Geld braucht. “

Klein macht dafür zum einen die Anti-Korruptionsgesetze wie etwa das Prinzip der „Mithaftung“ (renzasei, s.o.) dafür verantwortlich, dass japanische Politiker weniger Geld ausgeben. Illegale Aktivitäten wie Stimmenkauf hätten deshalb abgenommen. Bis auf wenige Kandidaten halten sich wohl die meisten Politiker auch an diese Bestimmungen. Zum anderen haben auch viele Großspender, sowohl wegen der zurückliegenden Skandale als auch wegen der Wirtschaftslage, ihre Zuwendungen reduziert. Das gilt vor allem für Spenden an die zentralen Parteiorganisationen, die die fehlenden Einnahmen nur zum Teil durch staatliche Parteienfinanzierung kompensieren können. ${ }^{392}$

Die staatliche Finanzierung machte in den vergangenen Jahren nur etwa ein Zehntel der Gesamteinnahmen der Parteien aus. Von den rund 31 Mrd. Yen staatlicher Parteienfinanzierung erhielt die LDP 2000 über ein Drittel (14,612 Mrd. Yen), die Demokratische Partei 7,66 Mrd. Yen. ${ }^{393}$ Parteien wie die SDPJ werden vermutlich in Zukunft stärker von öffentlichen Mitteln abhängig sein als die LDP oder andere konservative Parteien, die weiterhin auf (Unternehmens-) Spenden angewiesen sind. Nur wenige Parteien wie die Kômeitô oder die KPJ, die im Übrigen aus moralisch-ethischen Gründen auf staatliche Zahlungen verzichtet, können mit einer höheren Summe an Mitgliedsbeiträgen rechnen oder verfügen sogar über eigene Unternehmen. So finanziert sich die KPJ, die nominell über die höchsten Einnahmen der japanischen Parteien verfügt, vor allem über die Parteizeitung „Akahata“ (Rote Fahne). Allerdings müssen bei der LDP noch die Einnahmen der so genannten „Staatsbürgerlichen Vereinigung“ (kokumin seiji kyôkai) hinzugerechnet werden. ${ }^{394}$

Allgemein scheinen die Verbindungen zwischen den einzelnen Parteien und Interessengruppen schwächer geworden zu sein. Viele Interessengruppen überdachten ihr Spendenverhalten in der Zeit nach 1994 neu. So stellen die neuen konservativen Parteien für die LDP durchaus

\footnotetext{
${ }^{391}$ Vgl.: Köllner, Patrick: Drei Jahre nach den politischen Reformen - Eine Zwischenbilanz, Teil 1: Das Wahlgesetz und seine Auswirkungen, a.a.O., S. 171.

${ }^{392}$ Vgl.: Klein, Axel: A.a.O., S. 244ff.

${ }^{393}$ Vgl.: Asahi Shinbun: Niokuhassenman-en jimin wa gengaku, in: Asahi Shimbun, 28.06.2000, S. 4. (Asahi Shinbun: 280 Millionen: Die LDP hat nachgelassen.)

${ }^{394}$ Vgl.: Ebd., S. 276; Kagaya, Katsuo: A.a.O., S. 104-105., Köllner, Patrick: Parteienfinanzierung in Japan: Regulierungsmechanismen, a.a.O., S. 154.
} 
eine Konkurrenz bei der Einwerbung von Wirtschaftsspenden dar. Umgekehrt gab es Versuche seitens der LDP, engere Verbindungen zu einzelnen Gewerkschaften herzustellen. ${ }^{395}$

Für die Einzelpolitiker haben Spenden bei der Finanzierung der Wahlkreisaktivitäten nach wie vor große Bedeutung. Viele Politiker haben schließlich einen Ausweg gefunden, um das Verbot von Unternehmensspenden zu umgehen, indem sie entweder neue Ortsgruppen ihrer Partei gründeten oder offizielle Parteiorganisationen mit ihren eigenen Unterstützerorganisationen verschmolzen. Die lokalen LDP-Organisationen erhielten 1996 viermal so viele Spenden wie 1995. Diese Spenden, die früher direkt an die einzelnen Kandidaten gingen, werden nun offiziell als Spenden für die Partei gewertet, gehen im Wesentlichen aber wohl doch an die Kandidaten. ${ }^{396}$

Nebenbei sei hier erwähnt, dass die Zahl der registrierten Korruptionsvorfälle - wie erhofft tatsächlich zurückging. Zwischen 1993 und 1999 sank die Zahl der Verstöße im Wahlkampf um 71\%. Allerdings können die Zahlen auch auf einer geringen Aufdeckung von Korruptionsvorfällen beruhen, da die Gesetze relativ neu sind. Bruns geht davon aus, dass sich aufgrund einer rigideren Gesetzgebung nicht automatisch die tradierten Formen politischer Handlungsweisen ausschalten lassen. ${ }^{397}$

Allerdings sprach der befragte Unterhausabgeordnete Ôno tatsächlich von einer zunehmenden Transparenz bei der Finanzierung politischer Aktivitäten. Dies sei eine positive Folge der politischen Reformen. Auch Christensen merkt an, dass es für „Skandal-Politiker“ unter dem neuen Wahlsystem schwieriger geworden sei, wiedergewählt zu werden. ${ }^{398}$

Diese Erfahrung machte der Interviewpartner Funada: „[...] Also in meinem Fall gab es ja einen kleinen Skandal und ich wurde einmal abgewählt, nicht wahr. Wenn man also nachlässig wird, der Ruf der LDP ist ja auch etwas schlechter geworden, dann wird man unter dem Einerwahlsystem wahrscheinlich eher verlieren. “399 In diesem Fall handelte es sich allerdings nicht um einen Finanzskandal, sondern lediglich um eine außerehelche Affäre mit einer Oberhausabgeordneten.

Abschließend lässt sich sagen, dass große Hoffnungen in die 1994 verabschiedeten Reformen gesetzt worden waren. Diese haben sich jedoch nur zu einem geringen Teil erfüllt, gerade was die nach wie vor personalisierten Wahlkämpfe betrifft. Dazu sagt Park Cheol-Hee in seiner Studie zu den Auswirkungen der politischen Reformen: „Einfach ausgedrückt gibt es eine gro-

\footnotetext{
395 Jain, Purnendra C.: Party Politics at the Crossroads, a.a.O., S. 25.

${ }^{396}$ Vgl.: Asahi Shinbun: Niokuhassenman-en jimin wa gengaku, a.a.O., S. 4.

${ }^{397}$ Vgl.: Bruns, Gabriele: A.a.O., S. 261.

${ }^{398}$ Vgl.: Christensen, Raymond V.: The New Japanese Election System, a.a.O., S. 60.

${ }^{399}$ In diesem Fall handelte es sich allerdings nicht um einen Finanzskandal, sondern lediglich um eine außerehelche Affäre mit einer Oberhausabgeordneten.
} 
ße Lücke, die zwischen den anfänglichen Erwartungen an die Reform des Wahlsystems und der Wirklichkeit klafft. “400

\subsubsection{Soziale Hintergründe japanischer Abgeordneter nach 1993}

\subsubsection{Berufsfelder}

Der Blick auf die beruflichen Hintergründe japanischer Unterhausabgeordneter nach den politischen Reformen zeigt, dass es kurz nach Einführung des neuen Wahlsystems 1996 (s. 6.2), mit einer Ausnahme, keine grundlegenden Veränderungen gegeben hat. Die wichtigsten Rekrutierungsfelder stellten 1996 immer noch die Regionalpolitiker sowie die Sekretäre von Abgeordneten dar. Fast 40\% der Unterhausabgeordneten in Japan rekrutierten sich aus diesen beiden Berufen. Auch die Zahl der ehemaligen Beamten blieb stabil, deren Anteil an den Abgeordneten erhöhte sich durch die Verkleinerung des Parlaments aber etwas.

Ein wichtiger Unterschied zu der Zeit vor 1993 ist allerdings, dass ehemalige Spitzenbeamte nun auch unter den Abgeordneten der Oppositionsparteien, z.B. der damaligen Shinshintô, zu finden sind. Während des 1955er-System war es eher selten, dass ehemalige Ministerialbeamte für Oppositionsparteien antraten, wie einige Abgeordnete in den Interviews anmerkten. Hier hat es innerhalb einzelner Berufsfelder also durchaus Veränderungen gegeben.

Ließen sich vor 1993 noch vier typische prä-professionelle Berufsfelder japanischer Unterhausabgeordneter identifizieren - neben den ehemaligen Beamten, Regionalpolitikern und Sekretären von Abgeordneten die Gruppe der Gewerkschaftsfunktionäre und -Mitarbeiter - verlor das letztgenannte Berufsfeld an Bedeutung. Das lässt sich wohl auf die hohen Mandatsverluste der linken Parteien in Japan zurückführen.

Viertgrößte Gruppe stellten 1996 die ehemaligen Parteifunktionäre und -mitarbeiter dar. Diese fanden sich vor allem in den Oppositionsparteien KPJ und Shinshintô, für die zu jener Zeit auch die Kandidaten der Kômeitô antraten, unter denen der Anteil der Parteimitarbeiter relativ hoch war. In der DPJ, damals mit 52 Sitzen noch relativ klein, ließen sich noch keine typischen Rekrutierungsfelder erkennen.

\subsubsection{Die Zahl der Erbabgeordneten}

Interessant ist aber vor allem ein Blick auf die Zahl der Erbabgeordneten seit Inkrafttreten der politischen Reformen. Zum Zeitpunkt der Fertigstellung des Manuskripts hat es in Japan drei Unterhauswahlen (1996, 2000, 2003) gegeben. Nach der letzten Wahl unter dem alten Wahlsystem verteilten sich die Erbabgeordneten wie folgt:

\footnotetext{
${ }^{400}$ Park, Cheol Hee: A.a.O., S. 12.
} 
Abbildung 14: Erbabgeordnete nach der Wahl 1994 (40. Legislaturperiode)

\begin{tabular}{|c|c|c|c|c|c|c|c|c|}
\hline & LDP & SDPJ & JRP & JNP & NPS & DSP & Sonstige & Gesamt \\
\hline Abgeordnete & 200 & 70 & 62 & 33 & 21 & 15 & 29 & 511 \\
\hline Erbabgeordnete & 89 & 7 & 13 & 3 & 6 & 5 & 8 & 131 \\
\hline 2. Generation & 75 & 7 & 11 & 2 & 5 & 5 & 6 & 112 \\
\hline 3. Generation & 14 & - & 2 & 1 & - & - & 1 & 17 \\
\hline 4. Generation & - & - & - & - & 1 & - & 1 & 2 \\
\hline $\begin{array}{l}\text { Anteil der Erbab- } \\
\text { geordneten }\end{array}$ & $44,5 \%$ & $10 \%$ & $21 \%$ & $9,1 \%$ & $28,6 \%$ & $33,3 \%$ & $27,6 \%$ & $25,6 \%$ \\
\hline
\end{tabular}

Nach: Blechinger, Verena: Politische Korruption in Japan, Institut für Asienkunde, Hamburg 1998, S. 102.

Doch auch nach der Einführung des neuen Wahlsystems ist die Zahl der Erbabgeordneten im Unterhaus relativ stabil geblieben.

\section{Abbildung 15: Erbabgeordnete nach der Wahl 2000 (42. Legislaturperiode)}

(in Klammern: Zahl der weiblichen Erbabgeordneten)

\begin{tabular}{l||c|c|c|c|c|c|c|c|c||c} 
Partei & LDP & DPJ & SDPJ & CGP & KPJ & LP & NCP & Sonst. & Unabh. & Gesamt \\
\hline \hline Abgeordnete & 240 & 124 & 18 & 31 & 20 & 22 & 7 & 2 & 12 & $476^{401}$ \\
\hline Erbabgeordnete & $98(4)$ & $24(1)$ & $1(1)$ & 2 & - & 7 & 1 & - & 4 & $137(6)$ \\
\hline \multicolumn{1}{c|}{ 2. Generation } & 80 & 21 & 1 & 2 & - & 6 & 1 & - & 4 & 115 \\
\hline \multicolumn{1}{c|}{ 3. Generation } & 13 & 2 & - & - & - & 1 & - & - & - & 16 \\
\hline ab 4. Generation & 5 & 1 & - & - & - & - & - & - & - & 6 \\
\hline \hline $\begin{array}{l}\text { Anteil der Erb- } \\
\text { abgeordneten }\end{array}$ & $40,8 \%$ & $19,3 \%$ & $5,5 \%$ & $6,4 \%$ & - & $31,8 \%$ & $14,3 \%$ & - & $33,3 \%$ & $\mathbf{2 8 , 8} \%$
\end{tabular}

Nach: Saitô, Keiko: Seikanyôran, Vol. 20, No. 47, Seisakujihô Ai Pi, Tôkyô 2002, Sonderbeilage S. 2ff. (Saitô, Keiko: Führer durch Politik und Bürokratie.), eigene Berechnungen.

Zwischen 1994 und 2000 scheint sich der Rekrutierungspfad des Erbabgeordneten behauptet zu haben. Der Anteil der Erbabgeordneten im Unterhaus ist sogar leicht gestiegen. Er ist auch

${ }^{401}$ Vier Sitze waren zum Zeitpunkt der Veröffentlichung vakant. 
weiterhin kein ausschließliches Phänomen der LDP, sondern ist ebenso in den neuen Oppositionsparteien zu finden. Bei der Betrachtung der Zahlen muss allerdings stets bedacht werden, dass es vor allem in den 1990er Jahren zu einer Reihe von Parteigründungen, -abspaltungen und -austritten kam.

Abbildung 16: Ergebnis der Unterhauswahl 2003 (aktuelle Sitzverteilung 2004)

\begin{tabular}{|c|c|c|c|c|c|c|c|c|c|}
\hline Partei & LDP & DPJ & CGP & KPJ & SDPJ & $\begin{array}{c}\text { Jiyû } \\
\text { rengô }\end{array}$ & Sonst. & Unabh. & Gesamt \\
\hline Direktmandat & 168 & 105 & 9 & 0 & 1 & 1 & 5 & 11 & 300 \\
\hline \multirow[t]{2}{*}{ Listenplatz } & 69 & 72 & 25 & 9 & 5 & - & - & - & 180 \\
\hline & 237 & 177 & 34 & 9 & 6 & 1 & 5 & 11 & \\
\hline Gesamt & (249) & (176) & (34) & (9) & (6) & (1) & $(-)$ & (5) & 480 \\
\hline
\end{tabular}

Quelle: Saitô, Keiko: Seikanyôran, Vol. 22, No. 50/2003, Seisakujihô Ai Pi, Tôkyô 2004.

Sonstige: NCP und Mushozoku-no kai („Gemeinschaft der Unabhängigen“)

In den Unterhauswahlen 2003 konnte sich die LDP als stärkste Partei behaupten, auch wenn sie einige Sitze verlor. Ihr Koalitionspartner, die Kômeitô (CGP), konnte die Zahl der Mandate im Unterhaus sogar leicht ausbauen. Auf der Oppositionsseite hingegen gab es grundlegende Veränderungen. Die DPJ ist, vor allem durch Fusionen mit anderen konservativen Parteien, mit 176 Sitzen nun deutlich stärker als noch 2002, als sie nur über 124 Sitze verfügte. KPJ (2002: 20 Sitze) und SDPJ (2002: 18 Sitze) mussten große Verluste hinnehmen.

Bei der Unterhauswahl 2003 traten 1026 Kandidaten an, darunter 672 neue Kandidaten. Der Anteil der Frauen ist nach wie vor sehr gering und lag bei etwa 14,5\%. Nach wie vor sitzen mit 34 Frauen sehr wenig weibliche Abgeordnete im japanischen Unterhaus. Von Doppelkandidaturen sowohl auf der Parteiliste als auch im Einerwahlkreis machten vor allem die großen Parteien LDP (255 von 336 Kandidaten) und DPJ (264 von 277), aber auch die SDPJ und die KPJ Gebrauch. Die Kômeitô hingegen stellte, in Absprache mit der LDP, nur zehn Kandidaten in den Direktwahlkreisen auf, von denen immerhin neun gewählt wurden. Ansonsten setzte die Kômeitô auf Parteilisten, auf denen 133 Politiker kandidierten. Der Wahlkampf in den Einerwahlkreisen war also im Wesentlichen von der Auseinandersetzung zwischen Kandidaten der LDP und der DPJ gekennzeichnet, auch wenn die KPJ aus Prinzip in jedem Wahlkreis einen 
Kandidaten aufstellte. Beobachter sprachen deshalb von einer Tendenz zu einem ZweiParteien-System. ${ }^{402}$

Rekrutierungsbasis der LDP bildeten in erster Linie nach wie vor die Abgeordneten der Regionalparlamente, Beamte und Sekretäre von Abgeordneten. Auf Seiten der Opposition haben allerdings die Gewerkschaften durch die hohen Stimmenverluste der SDPJ ihre Rolle als Rekrutierungsfeld eingebüßt, dafür hat die Zahl der ehemaligen Parteifunktionäre und -mitarbeiter zugenommen. Die Zahl der ehemaligen Beamten unter neuen Kandidaten sank laut Yomiuri Shinbun in den letzten Jahren aufgrund einiger Skandale und eines schlechten Images. Der Anteil der meisten anderen Berufsgruppen ist hingegen überraschend stabil geblieben. ${ }^{403}$

\section{Abbildung 17: Erbabgeordnete nach der Wahl 2003 (43. Legislaturperiode)}

(in Klammern: Zahl der weiblichen Erbabgeordneten)

\begin{tabular}{r||c|c|c|c|c|c|c||c}
\multicolumn{1}{c|}{ Partei } & LDP & DPJ & SDPJ & CGP & KPJ & Jiyûrengô & Unabh. & Gesamt \\
\hline \hline Abgeordnete & 249 & 176 & 6 & 34 & 9 & 1 & 5 & 480 \\
\hline Erbabgeordnete & $103(4)$ & $30(2)$ & - & 2 & - & - & $1(1)$ & $136(7)$ \\
\hline 2. Generation & 84 & 28 & - & 2 & - & - & 1 & 115 \\
\hline 3. Generation & 14 & 1 & - & - & - & - & - & 15 \\
\hline \multicolumn{1}{c|}{ ab 4. Generation } & 5 & 1 & - & - & - & - & - & 6 \\
\hline \hline $\begin{array}{l}\text { Anteil der Erbab- } \\
\text { geordneten }\end{array}$ & $41,4 \%$ & $17 \%$ & - & $5,9 \%$ & - & - & $20 \%$ & $\mathbf{2 8 , 5 \%}$ \\
\hline
\end{tabular}

Nach: Saitô, Keiko: Seikanyôran, Vol. 22, No. 50/2003, Seisakujihô Ai Pi, Tôkyô 2004 (Saitô, Keiko: Führer durch Politik und Bürokratie.), eigene Berechnungen.

Unter den Kandidaten der Unterhauswahl 2003 befanden sich auch 150 Erbpolitiker, von denen 136 den Einzug ins Parlament schafften. ${ }^{404}$ Der Anteil der Erbabgeordneten ist mit 28,5\% nach wie vor relativ hoch. Die Verteilung auf die verschiedenen Generationen ist ebenfalls stabil geblieben. Bemerkenswert ist lediglich die Fusion von LP und DPJ, durch die sich die Zahl der Erbabgeordneten zwar absolut erhöhte, relativ durch den Mandatszuwachs der Partei aber sank.

\footnotetext{
${ }^{402}$ Vgl.: Japan aktuell: Die Unterhauswahl vom November 2003 im Überblick, a.a.O., S. 530-531.

403 Vgl.: Yomiuri Shinbun: Seidaikotai-mo seshû nezuyoku, in: Yomiuri Shinbun, 14.06.2000, S. 4. (Yomiuri Shinbun: Generationswechsel und Erbfolge tief verwurzelt.); Abb. 22.

${ }^{404}$ Vgl.: Japan aktuell: Die Unterhauswahl vom November 2003 im Überblick, a.a.O., Abb. 16.
} 
Offensichtlich haben sich die Erbabgeordneten unter dem neuen Wahlsystem behaupten können. Dieser Rekrutierungspfad scheint nach wie vor eine große Rolle vor allem in der LDP zu spielen. In den folgenden Abschnitten sollen daher die Entwicklungen auf der Nachfrage- und auf der Angebotsseite bei der politischen Rekrutierungen untersucht werden. 


\subsection{Die Veränderungen auf der Nachfrageseite}

\subsubsection{Der Übergang zum neuen Wahlsystem}

Nach Inkrafttreten der neuen Bestimmungen stellte zunächst die neue Wahlkreiseinteilung ein Problem dar. Durch die Umwandlung der Mehrerwahlkreise in Einerwahlkreise fiel ein Teil der Mandate weg. Aus 129 Wahlkreisen mit insgesamt 511 Mandaten wurden 300 Einerwahlkreise. Hinzu kamen zunächst noch 200 Mandate über die Parteilisten, die später auf 180 verringert wurden.

Die Schwierigkeiten bei der Kandidatenaufstellung in der LDP werden bei Park anschaulich beschrieben. ${ }^{405}$ Generell galt, dass Amtsinhaber ein Anrecht auf eine Kandidatur in ihrem Wahlkreis hatten. Verzichtete ein Mandatsträger auf die Kandidatur im Direktwahlkreis, bekam er den Vorzug auf der Parteiliste, wobei die Parteizentrale über die endgültige Reihenfolge entschied. Hinter diesen Kandidaten sollten auf der Parteiliste die Kandidaten platziert werden, die in „unsicheren Wahlkreisen“ antraten. Für die Reihenfolge wurden die Wahlergebnisse der zurückliegenden Wahlen berücksichtigt. Wer im Verhältnis zum Sieger in seinem Wahlbezirk das beste Ergebnis hatte, bekam den besseren Listenplatz.

Park nennt als Beispiel den ehemaligen Wahlkreis Tôkyô 10, der aus drei Bezirken bestand, die nun zu Einerwahlkreisen wurden. ${ }^{406}$ Bisher gab es im Wahlkreis Tôkyô 10 vier Abgeordnete, die beiden LDP-Abgeordneten Shimamura und Kujiraoka sowie den KPJ-Abgeordneten Sasaki und den Politiker Kamoshita von der Nihon Shintô. Während Shimamura vor allem im Bezirk Edogawa-ku seine Anhänger hatte, war Kujiraokas Hochburg der Bezirk Adachi-ku (jetzt Tôkyô 17). So war es voraussehbar, dass Shimamura sich den Bezirk Edogawa aussuchte und auch erhielt. Kujiraoka jedoch verzichtete auf eine Kandidatur im Bezirk Adachi-ku, zum einen aufgrund seines hohen Alters, er war fast 80 Jahre alt, zum anderen weil die beiden Abgeordneten der Opposition dort kandidierten. Kujiraoka erhielt deshalb Platz zwei auf der LDPListe des Tôkyô-Blocks. Interessant ist in diesem Zusammenhang auch die Besetzung der übrigen Listenplätze. Der Vorsitzende der Parteiorganisation von Tôkyô erhielt den ersten Listenplatz, vermutlich weil ihm mit dem DPJ-Spitzenpolitiker Hatoyama Kunio in seinem Wahlbezirk ein äußerst schwieriger Gegner gegenüber stand. Der dritte Platz ging an den Politiker Takashi, der gerade aus der Shinshintô zurückgekehrt war. Auf den vierten Platz kamen die restlichen elf Mandatsträger aus Tôkyô und auf den 15. Platz wurden die dreizehn neuen Kandidaten der LDP gesetzt. Während in einigen Fällen der Listenplatz als Sicherheitsnetz diente, war er in anderen Fällen eine Art Rückzugsposition für ältere Amtsinhaber. Dies bewertet Park auch als

\footnotetext{
${ }^{405}$ Vgl.: Park, Cheol-Hee: A.a.O., S. 47f.

${ }^{406}$ Vgl.: Ebd., S. 47ff.
} 
eine Art Beitrag zum Generationswechsel in der Partei. Die LDP konnte nun für den frei gewordenen Wahlkreis einen neuen, jüngeren Kandidaten suchen. ${ }^{407}$

Abgesehen davon, dass sich die Suche nach einem Nachfolger für Kujiraoka als sehr schwierig herausstellte, war die oben genannte Lösung nicht in allen Wahlkreisen möglich, gerade dann, wenn es mehr Mandatsträger als Wahlkreise gab. Die Kandidaten wollten verständlicherweise auch weiterhin in Wahlkreisen antreten, die zumindest teilweise den alten entsprachen, um ihre lokalen Netzwerke nutzen zu können. Klein nennt hier den Viererwahlkreis Gumma 3, der in zwei Einerwahlkreise unterteilt wurde. Dort gab es drei LDP-Abgeordnete und einer von ihnen, der ehemalige Ministerpräsident Nakasone, weigerte sich, auf die Parteiliste zu wechseln, obwohl er aufgrund seines Alters einen sicheren Platz bekommen hätte. Offensichtlich wollte er seinen Wahlkreis später an seinen Sohn vererben. Nach heftigen Auseinandersetzungen ließ er sich von Ministerpräsident Hashimoto zu einer „lebenslangen“ Kandidatur auf Platz eins der Parteiliste des Kita-Kantô-Blocks überreden. Solche Konflikte sorgten dafür, dass in einigen Fällen die Kandidatenbestimmung verzögert wurde und viele Kandidaten nicht wie gewünscht schon ein Jahr vor der Wahl mit dem Wahlkampf beginnen konnten. ${ }^{408}$

Ein Teil der Konflikte konnte überdies durch Absprachen und Ausweichpositionen auf den Parteilisten gelöst werden. Eine interessante Lösung ist das so genannte „Costa-Rica-System“ (kosutarika-hôshiki): „Bei dieser Vorgehensweise wechseln sich zwei Politiker der gleichen Partei, die beide den gleichen Einerwahlkreis für sich beanspruchen, in der Kandidatur dort ab. Tritt zunächst Politiker A als Direktkandidat an, weicht Politiker B auf die Parteiliste aus. Bei der nächsten Wahl verfahren beide umgekehrt. “409

Einer der Interviewpartner, Hamada Yasukazu, war von dieser Regelung betroffen und stellte in dieser Hinsicht einen besonders interessanten Fall dar. Zur Zeit des Interviews wurde dieses System von der LDP in drei Wahlkreisen angewendet. Für die Kandidaten selbst hat diese Lösung weitreichende Folgen: Auf der einen Seite müssen beide Kandidaten zusammenarbeiten, um die nötige Stimmenzahl im Einerwahlkreis für die nächsten Wahlen zu sichern, auf der anderen Seite befinden sie sich in einer Konkurrenzsituation: „Je nachdem wie engagiert der andere Kandidat gewesen ist, entscheidet sich dann das nächste Mal die Stimmenzahl. Das heißt, sie entscheidet sich danach, wie beliebt der Partner ist. Auf der anderen Seite kommt durch die Konkurrenz ein gutes Ergebnis zu Stande. Bei meiner ersten Wahl hatte ich etwa

\footnotetext{
${ }^{407}$ Vgl.: Park, Cheol-Hee: A.a.O., S. 49.

${ }^{408}$ Vgl.: Klein Axel: A.a.O., S. $207 \mathrm{ff}$.

${ }^{409}$ Klein Axel: A.a.O., S. 284.
} 
100.000 Stimmen, aber weil die Zahl unter Herrn Nakamura auf 120.000 wuchs, freue ich mich darauf, dass meine Zahl nächstes Mal wachsen wird. “410

Hamada Yasukazu scheint mit dieser Regelung jedoch nicht zufrieden zu sein, da er sich im Verlauf des Interviews eindeutig gegen die Verhältniswahl aussprach. In diesem Sinne ist das „Costa-Rica-System“ sicherlich nicht mehr als eine Notlösung für die Übergangszeit gewesen. Neue Absprachen nach dieser Methode sollen den Plänen der LDP zufolge nicht mehr zugelassen werden. ${ }^{411}$

Generell entschied in Streitfällen über eine Kandidatur die Parteiführung. Allerdings stellt sich die Frage, welche Sanktionsmöglichkeiten die Parteien hatten (bzw. haben). Starke Kandidaten können die Partei mit ihrer kôenkai verlassen oder zumindest damit drohen. Ein weiteres Problem, vor dem die LDP vor der Unterhauswahl 1996 stand, war, dass 14 Wahlkreise zunächst unbesetzt blieben. Neben zwei Wahlkreisen, die aus wahltaktischen Gründen (Absprachen mit Koalitionspartnern) frei blieben, waren es vor allem Wahlkreise, in denen Kandidaten der Opposition bisher besonders stark gewesen waren oder es keinen geeigneten Kandidaten gab. Die LDP hielt in diesen Fällen Oppositionspolitikern, bei denen ein Parteiwechsel möglich schien, eine Hintertür offen, später in die LDP (zurück) zu kommen und stellte keinen Kandidaten auf. In anderen Fällen wurde sogar versucht, Oppositionspolitiker direkt abzuwerben. ${ }^{412}$

Ungeachtet aller Probleme der Kandidatenbestimmung, gab es auch einige Kandidaten, die die Neueinteilung der Wahlkreise für sich zu nutzen wussten. Politiker, die über besonders große und weit verzweigte Unterstützerorganisationen verfügten, teilten ihre kôenkai auf und übergaben sie Verwandten oder engen Mitarbeitern. Klein spricht in diesem Zusammenhang von „einer Art doppelten Mandatsgewinn“ “413.

\subsubsection{Vorwahlen und „öffentliche Ausschreibungen“ - Neue Formen der Rekrutierung?}

Die Rekrutierungsmechanismen und das Aufstellungsverhalten während der Übergangszeit zum „Grabensystem“ waren vor allem in der LDP recht uneinheitlich und variierten stark. Daran scheint sich bis heute nicht viel geändert zu haben. Im Folgenden soll der Fokus auf der Kandidatenrekrutierung bzw. der Erbfolge in den Einerwahlkreisen liegen, wo sich der Rekrutierungspfad des Erbabgeordneten offensichtlich gehalten hat. Betrachtet werden dabei vor allem die LDP und die DPJ.

\footnotetext{
${ }^{410}$ Hamada Yasukazu erreichte bei der folgenden Wahl 2003115.708 Stimmen.

${ }^{411}$ Vgl.: Japan aktuell: Gerangel in der LDP um Parlamentskandidatenmode geht weiter, in: Japan aktuell. Wirtschaft. Politik. Gesellschaft, Heft April/2003, S. 130.

${ }^{412}$ Vgl.: Park, Cheol-Hee: A.a.O., S. 52.

${ }^{413}$ Vgl.: Klein, Axel: A.a.O., S. 209.
} 
Die Bestimmung der Parteilisten soll ebenfalls kurz angesprochen werden, auch wenn hier eine Erbfolge für sehr unwahrscheinlich gehalten wird. Die Zahl der Erbabgeordneten, die über einen Listenplatz ins Unterhaus eingezogen sind, ist deutlich niedriger als die Zahl der Erbabgeordneten in den Einerwahlkreisen. Dennoch hat die Bestimmung der Parteilisten einige Tendenzen, der Kandidatenrekrutierung auf der Nachfrageseite verstärkt, z.B. zunehmende Zentralisierung bei der Kandidatenauswahl.

\section{Abbildung 18: Erbabgeordnete nach Direktmandat/Listenplatz}

\begin{tabular}{l||c||c|c} 
& $\begin{array}{c}\text { Abgeordnete } \\
\text { insgesamt }\end{array}$ & $\begin{array}{c}\text { 42. Legislaturperiode } \\
\mathbf{( 2 0 0 0 )}\end{array}$ & $\begin{array}{c}\text { 43. Legislaturperiode } \\
\mathbf{( 2 0 0 3 )}\end{array}$ \\
\hline \hline Direktmandat & 300 & $113(37,6 \%)$ & $112(37,3 \%)$ \\
\hline Listenplatz & 180 & $24(13,3 \%)$ & $24(13,3 \%)$ \\
\hline \hline & 480 & $137(28,5 \%)$ & $136(28,3 \%)$
\end{tabular}

Nach: Saitô, Keiko: Seikanyôran, Vol. 20, No. 47, Seisakujihô Ai Pi, Tôkyô 2002 (Saitô, Keiko: Führer durch Politik und Bürokratie.), Saitô, Keiko: Seikanyôran, Vol. 22, No. 50/2003, Seisakujihô Ai Pi, Tôkyô 2004 (Saitô, Keiko: Führer durch Politik und Bürokratie.), eigene Berechnungen.

Die offiziellen Bestimmungen zur Kandidatenaufstellung in der LDP sind auf den ersten Blick recht eindeutig. ${ }^{414}$ Demnach wird der Kandidat auf der lokalen Ebene bestimmt. Dieser Kandidatenvorschlag muss die regionale Parteiversammlung (Todôfuken shibu rengôkai) passieren und wird dann der Parteizentrale vorgelegt. Diese entscheidet dann nach Rücksprache mit dem örtlichen Wahlausschuss über die Kandidatur. Bei Konflikten zwischen der lokalen und der regionalen Ebene soll der Kandidat vor der regionalen Parteiversammlung vorsprechen, die dann eine Empfehlung an die Zentralpartei abgibt, wo der Kandidat endgültig bestimmt wird. Kandidaten, die sich negativ über die Parteipolitik äußern, können die Anerkennung als offizieller Kandidat auf Anweisung der Parteizentrale noch nachträglich verlieren.

Die endgültige Entscheidung über die Kandidaten liegt formell also auf der zentralen Ebene. Dazu gibt es noch einige ergänzende Regelungen. Generell haben Amtsinhaber ein Vorrecht

\footnotetext{
${ }^{414}$ Die folgenden Ausführungen entstammen den Bestimmungen der LDP zum Wahlkampf und zur Kandidatenaufstellung aus den Jahren 1994 und 2002.

Vgl.: Jiyûminshutô: Senkyotaisaku yôkô, heisei 6, 24.11. (Liberaldemokratische Partei: Prinzipien für die Wahlen, 24.11.1994); Jiyûminshutô: Kôhosha sentei kijun, heisei 6, 24.11. (Liberaldemokratische Partei: Grundsätze der Kandidatenbestimmung, 24.11.1994), Jiyûminshutô: Seitô to senkyo, heisei 14, 8.28. (Liberaldemokratische Partei: Partei und Wahlen, 28.08.2002.)
} 
auf eine Kandidatur. Für neue Kandidaten schlägt die Partei die Durchführung von „öffentlichen Ausschreibungen“ (kôhosha-kôbo) und Vorwahlen (yobisen) vor.

In der Realität jedoch wird die Kandidatenbestimmung in der LDP häufig als intransparent und schwer nachvollziehbar beschrieben. Abgesehen von der endgültigen Anerkennung auf zentraler Ebene, hat die LDP den Rekrutierungsprozess an sich den regionalen bzw. den lokalen Organisationen im Wahlkreis überlassen. Genaue Bestimmungen und Regeln zu den Auswahlverfahren existieren allerdings nicht. ${ }^{415}$

In diesem Zusammenhang ist wieder das von Park untersuchte Beispiel des Wahlkreises Tôkyô 17 interessant. Um einen Kandidaten für den frei gewordenen Wahlkreis zu finden, wurden zwar Interessenten aufgerufen, sich zu melden. Die Auswahl des Kandidaten wurde jedoch „nicht öffentlich“ von einer Jury vorgenommen, die im Wesentlichen aus Vertretern verschiedener Interessengruppen bestand und deshalb jemanden „aus den eigenen Reihen“ wählte: „In Japan ist es häufig so, dass die Auswahl [des Kandidaten - d. A.] nicht öffentlich und intransparent ist. Ein Charakteristikum der Auswahl in Japan ist, dass die Meinung lokal einflussreicher Leute von großer Bedeutung ist und die Auswahl, ohne standardisierte Regeln im Auswahlprozess, dem einfachen Wähler verborgen bleibt. “416 Dem Wähler wird lediglich das Ergebnis präsentiert, wohingegen die Kandidatenbestimmung in einem relativ geschlossenen Personenkreis vorgenommen wird.

Auch Bruns konnte noch keinen Wandel zu neuen Rekrutierungsprozessen feststellen. Statt dessen würde die Kandidatenbestimmung „lang eingeübten“ Mustern folgen. ${ }^{417}$ Ähnlich sah es ein Teil der befragten LDP-Abgeordneten, wobei es sich hierbei um die Abgeordneten handelte, die schon besonders lange in der Politik sind (Hori, Ôno, Ishiba). Diese Abgeordneten waren der Meinung, dass kein grundlegender Wandel statt gefunden habe, auch wenn es in einigen Bereichen der Kandidatenbestimmung Änderungen gab. Die endgültige Entscheidung über die Kandidatur liege nach wie vor auf zentraler Ebene, die Prozesse selbst hätten sich auch kaum gewandelt. Hori und Ôno nannten in diesem Zusammenhang die Möglichkeit zur Selbstnominierung von Kandidaten, die keine offizielle Kandidatur erhalten, bei einer erfolgreichen Wahl aber in die Partei zurückkehren können. Dies scheint noch immer eine Möglichkeit für Kandidaten darzustellen, die aus den verschiedensten Gründen mit der Kandidatenbestimmung nicht einverstanden sind. ${ }^{418}$

Allerdings gibt es hierzu auch andere Meinungen. Der Abgeordnete Itô beispielsweise betonte die Wichtigkeit einer offiziellen Kandidatur. Unter dem aktuellen Wahlsystem sei es von

\footnotetext{
${ }^{415}$ Vgl.: Park, Cheol-Hee: A.a.O., S. 56f.

${ }^{416}$ Park, Cheol-Hee: A.a.O., S. 58.

${ }^{417}$ Vgl.: Bruns, Gabriele: A.a.O., S. 272f.

${ }^{418}$ Vgl.. Klein, Axel: A.a.O., S. 210f.; Park, Cheol-Hee: A.a.O., S. 51f.
} 
großer Wichtigkeit, als offizieller Kandidat einer Partei antreten zu können. Schließlich braucht ein Kandidat heute die Mehrheit der abgegebenen Stimmen für ein Mandat. Eine Selbstnominierung bietet sich also nur für die Kandidaten an, die in ihren Wahlkreisen besonders stark sind und über einflussreiche Unterstützerorganisationen verfügen. Die beiden Abgeordneten Ôno und Hori gehören zu dieser Gruppe und haben beide bei der letzten Wahl fast dreimal so viele Stimmen bekommen wie der jeweils Zweitplatzierte in ihrem Wahlkreis.

In der DPJ werden in einem größeren Umfang als in der LDP Vorwahlen zur Kandidatenbestimmung durchgeführt. Unter diesem Druck hat die LDP ihre Parteiorganisationen jüngst angewiesen, die Kandidaten anstehender Nachwahlen ebenfalls öffentlich, also durch „öffentliche Ausschreibungen“ (kôhosha-kôbo) und Vorwahlen (yobisen), zu bestimmen. Besonders die Abgeordneten der Präfekturparlamente fordern transparente Vorwahlen, da sie auf Grund der „Privatisierung der Wahlkreise “ 419 durch Erbabgeordnete kaum Chancen haben, auf die nationale Ebene zu wechseln. Die Mehrheit der befragten LDP-Abgeordneten wies auf eine Zunahme dieser Rekrutierungsformen hin, auch wenn diese Systeme noch nicht Praxis in ihren eigenen Wahlkreisen sind. Lediglich der Abgeordnete Itô musste sich in einer Vorwahl stellen.

Während bei einer „öffentlichen Ausschreibung“ Interessenten aufgerufen werden, sich vor einem Auswahlgremium als Kandidat zu bewerben, werden in den lokalen Parteiorganisationen auch zunehmend Vorwahlen durchgeführt, wenn es mehrere Bewerber gibt. Das heißt, es wird z.B. unter den Parteimitgliedern eine Vorwahl durchgeführt und der ausgewählte Kandidat der Parteizentrale weiterempfohlen, die ihn dann aufstellt. ${ }^{420}$ Allerdings wies der Abgeordnete Hamada an diesem Punkt darauf hin, dass nicht klar geregelt sei, ob und in welcher Form beim Rücktritt eines Kandidaten eine Vorwahl durchgeführt werden soll.

Bisher hat die LDP erst in wenigen Fällen, z.B. bei Vakanzen in den Präfekturen Saitama und Miyagi, Vorwahlen durchgeführt. Dieses System scheint sich aber vor allem dort zu bewähren, wo die LDP eher schwach ist, wie etwa in städtischen Wahlkreisen. ${ }^{421}$ Beispiel für die Durchführung einer „öffentlichen Ausschreibung“ bietet der Wahlkreis Miyagi 2, der in der Präfekturhauptstadt Sendai liegt. Dort stand eine Nachwahl an und die LDP rief Interessenten auf, sich zu melden und vor dem Regionalausschuss der Partei zur Wahl zu stellen. Allerdings verließ der unterlegene Kandidat Kikuchi, ein Abgeordneter des Präfekturparlaments, daraufhin die Partei und trat als unabhängiger Kandidat an.

\footnotetext{
${ }^{419}$ Asahi Shinbun: Kôbo, yobisen, ze-ka hi-ka, Asahi Shinbun, 8.7.2003, S. 4 (Asahi Shinbun: Öffentliche Ausschreibungen, Vorwahlen: ja oder nein?)

${ }^{420}$ Vgl. Interview mit Funada Hajime im Anhang.

${ }^{421}$ Vgl.: Matsuda Takaji: Kohôsha jinzainan, in: Mainichi Shinbun, http://www.mainichi-

msn.co.jp/shakai/wadai/archive/news/2005/05/16/20050516ddm005070043000c.html (Matsuda Takaji: Qualitätsschwierigkeiten bei den Kandidaten)
} 
An diesem Punkt sahen die Interviewpartner ein Problem, selbst wenn sie sich grundsätzlich für die Durchführung von Vorwahlen aussprachen. In der LDP gebe es durchaus die Absicht, Rekrutierungsprozesse transparenter und offener zu gestalten, genaue Vorgabe zur Durchführung fehlten aber. Je nach Wahlkreis werden unterschiedliche Methoden angewendet, die Regeln scheinen sich mitunter beträchtlich zu unterscheiden. Das führt dann im ungünstigsten Fall zu einer mangelnden Legitimation und Unterstützung des Kandidaten. Auch wenn die Wahl in Miyagi-ken von dem offiziellen LDP-Kandidaten Akiba gewonnen wurde, zeigte sich, dass mit der Entscheidung der Partei unzufriedene Politiker bzw. unterlegene Aspiranten immer noch die Möglichkeit zu einer Selbstnominierung haben, wenn sie über entsprechende Unterstützerorganisationen verfügen. Die mangelnden einheitlichen Bestimmungen verschärfen dieses Problem. Dazu meinte der Abgeordnete Hori: „[...] Wenn ich krank werden und dann plötzlich sterben sollte, wüsste ich nicht, ob danach eine Vorwahl durchgeführt oder man jemanden [als Nachfolger - d.A.] benennen würde. Es würden sicherlich viele Leute einen Anspruch erheben. Aber auch, wenn sich viele Leute melden würden, gibt es keine Regel, wie entschieden wird. [...] Wenn man allerdings eine Vorwahl durchführt, wüsste man wohl nicht, ob derjenige, der bei der Vorwahl durchgefallen ist, bei der Wahl dem Sieger wirklich hilft. Dieses Problem gibt es. “ Allerdings merkte Hori an, dass es wohl in den Städten eher zu Veränderungen und transparenten Auswahlverfahren kommen würde als in Wahlkreisen wie seinem, der noch vom „alten Stil“ geprägt sei.

Ein anderes Problem, gerade bei einer Vorwahl, ist das Auswahlgremium: „Eine Vorwahl ist ganz gut, aber wenn man auf das Auswahlverfahren in der LDP schaut, unterscheidet sich diese Art der Vorwahl von der bei den US-Präsidentschaftswahlen. Zum ersten gibt es wenig Parteimitglieder. Kandidat wird man nicht, indem das politische Programm gut zur „Plattform“ passt. Kandidat wird man, je nach Region, abhängig von den Beziehungen, die man hat. Die Vorwahl wird von den Mitgliedern der LDP vorgenommen. Dieser Kreis ist aber nicht so groß und es gibt viele Karteileichen, daher ist die Wahlbeteiligung ziemlich gering. Wenn eine Primary mit einigen zehn Stimmen durchgeführt wird, kann man nicht sagen, dass sie fair ist“, so der Abgeordnete Itô.

Etwa die Hälfte der befragten LDP-Abgeordneten war zwar der Meinung, dass diese Art der Rekrutierung zunimmt, derzeit aus den oben genannten Gründen jedoch nur in wenigen Fällen Sinn macht. Funada Hajime merkte an, dass nur 10\% seiner kôenkai-Mitglieder in der LDP sind. Dieser „gap“ sei einer der größten Schwachpunkte der LDP. Wie das Beispiel Miyagi-ken gezeigt hat, ist es durchaus möglich, dass der unterlegene Kandidat das Ergebnis nicht anerkennt und mit seiner kôenkai einfach als unabhängiger Kandidat antritt. Diese Stimmen fehlen 
dann natürlich dem offiziellen Kandidaten. Andererseits sind viele Partei-Mitglieder Angehörige oder Angestellte von landwirtschaftlichen Genossenschaften und anderen Vereinigungen, die bestimmte Politiker unterstützen. Es ist daher kaum vorstellbar, dass ein „Außenseiter“ große Chancen hat, sich bei einer solchen Vorwahl unter den Parteimitgliedern durchzusetzen. $^{422}$

Abgesehen von der der KPJ, die ja wie eingangs erwähnt über relativ ausgeprägte lokale Organisationen verfügt, unterschied sich zumindest in der Übergangszeit zum neuen Wahlsystem die Kandidatenrekrutierung in den Oppositionsparteien nicht groß von der LDP. In den anderen konservativen Parteien, wie etwa der Shinshintô, die im wesentlichen aus der LDP hervorgegangen waren, wurde an überkommenen Rekrutierungsmechanismen festgehalten. Die meisten Kandidaten nominierten sich selbst bzw. behielten ihren alten Wahlkreis, andere Wahlkreise wurden durch informelle Absprachen oder durch die Parteiführung besetzt. Auch die DPJ unterschied sich zunächst nicht groß von den anderen Parteien. ${ }^{423}$

Inzwischen hat die DPJ, was neue Rekrutierungsformen wie Vorwahlen und „öffentliche Ausschreibungen“ betrifft, eine gewisse Vorreiterrolle übernommen. Interessenten werden aufgerufen, sich einem Auswahlgremium der regionalen Parteiorganisation zu stellen. Die Kandidaten werden dann der Parteizentrale weiterempfohlen, wo über die Aufstellung entschieden wird. $^{424}$ In der DPJ werden diese Methoden zur Kandidatenbestimmung wesentlich häufiger, aber nicht flächendeckend, angewendet als in der LDP. Bei der letzten Wahl hat die Oppositionspartei in 24 Wahlkreisen ihre Kandidaten auf diese Art bestimmt. ${ }^{425}$

Als erste Partei, so der Abgeordnete Matsuno im Interview, habe die Nihon Shintô unter Hosokawa, dessen Sekretär er war, die Form des kôhosha-kôbo („öffentliche Ausschreibung der Kandidatur“) angewandt und damit jenen eine Chance zur Kandidatur gegeben, die in der LDP „wegen der habatsu“ keine Chance gehabt hätten. Auf diesen Punkt wird später noch genauer eingegangen. Man muss aber an dieser Stelle berücksichtigen, dass die Auswahlgremien häufig aus den (regionalen) Abgeordneten der Partei und zusätzlich dem Parteivorsitzenden und dem Generalsekretär bestehen. Es werden aber auch Vorwahlen durchgeführt, bei denen die örtlichen Abgeordneten oder die örtlichen Parteimitglieder entscheiden. ${ }^{426}$

Terata Manabu bemerkte dazu: „Diesmal gab es bei mir in Akita zwar keine Vorwahl. Aber eine Vorwahl durchzuführen, ist sehr heikel, weil es ein großes Risiko gibt, dass niemand

\footnotetext{
${ }^{422}$ Vgl.: Japan aktuell: Gerangel in der LDP um Parlamentskandidatenmode geht weiter, a.a.O., S. 130.

${ }^{423}$ Vgl.: Bruns, Gabriele: A.a.O., S. $240 \mathrm{ff}$.

${ }^{424}$ Interview mit Terata Manabu.

${ }^{425}$ Vgl.: Matsuda Takaji: A.a.O.

${ }^{426}$ Interview mit Matsuno Yorihisa.
} 
kommt, wenn man sie durchführt [...] Man richtet schon vor der Vorwahl das Augenmerk auf eine Person. Man führt die Vorwahl durch, nachdem man schon vorher gesagt hat: ,Der ist es.' So bereitet man es vor. Wie bei einer Performance. Bei den Aufrufen sind zwar normale Leute, die signalisieren, dass sie kandidieren wollen oder es mal versuchen wollen. In letzter Zeit ist es ja einfacher geworden. Aber ich glaube, dass die Rekrutierungspfade tatsächlich noch sehr schmal ist."

Der Abgeordnete Watanabe sprach davon, dass es in seiner Partei einen Trend zu Vorwahlen gebe, die von den lokalen Parteiorganisationen durchgeführt werden. Es habe aber dennoch noch kein grundlegender Systemwechsel stattgefunden und die einzelnen Auswahlprozesse würden sich je nach Region unterscheiden. Insgesamt scheint es also auch in der DPJ trotz einiger Ansätze zu transparenten Rekrutierungsmechanismen noch keine einheitlichen Regelungen zu geben.

\subsubsection{Machtverschiebungen bei den Selektoren}

\subsubsection{Zwischen Faktion und Parteizentrale}

Auf der Seite der Selektoren hat es in der LDP offensichtlich einige Veränderungen gegeben, vor allem durch den relativen Machtverlust der habatsu. 1994 hatten die Faktionen in der LDP offiziell ihre Auflösung beschlossen und wandelten sich in so genannte benkyôkai (Studienkreise) um. Diese beschäftigten sich teilweise durchaus mit übergeordneten politischen Themen, in der Regel stellte diese Umwandlung aber nur eine formale Veränderung dar. Die habatsu wurden schon bald wiederbelebt und Abgeordnete und Regierungsmitglieder werden inzwischen wieder den einzelnen Faktionen zugerechnet. ${ }^{427}$ Tatsächlich existieren noch einige Studienkreise, wie das banchô seiji kenkyûsho („Banchô Politikforschungsinstitut“) der Kômura-ha, der etwa die befragten Abgeordneten Itô und Usui angehören. Die Aktivitäten dieses „Insituts“ beschränken sich aber im Wesentlichen auf die Veranstaltung von Fund-RaisingPartys. $^{428}$

An der Verteilung von Regierungsposten nach Proporz der habatsu scheint sich bis heute nichts geändert zu haben, was entsprechende Kritik hervorruft. ${ }^{429}$ Bemerkenswert ist aber, dass der aktuelle Parteivorsitzende und Ministerpräsident Koizumi keiner Faktion angehört und sich in einer Urwahl von den LDP-Mitgliedern wählen ließ. Krauss und Pekkanen sprechen davon, dass sich die Rolle der Faktionen geändert hat. Ihr Einfluss, etwa bei der Wahl des Par-

\footnotetext{
${ }^{427}$ Vgl.: Bruns, Gabriele: A.a.O., S. 268f.

${ }^{428}$ Auch einzelne Abgeordnete wie Funada Hajime verfügen über solche Studienkreise, deren Aufgabe vor allem die Fund-Raising-Organisation ist.

${ }^{429}$ Vgl.: Asahi Shinbun: Sôkaku-ni ,taika issô' atsuryoku, in:Asahi Shimbun, 28.06.2000, S. 4. (Asahi Shinbun: Der Druck auf die Regierung, die Postenanhäufung zu beseitigen.)
} 
teivorsitzenden, ist zwar noch vorhanden, wenn auch nicht mehr allein maßgeblich. Nach wie vor gibt es Auseinandersetzungen zwischen einzelnen Faktionen, wie die Abstimmung über die Postreform gezeigt hat (s.u.). Ihre Funktion im Bereich der Kandidatenaufstellung und der Wahlkampfhilfe für ihre Kandidaten haben die Faktionen jedoch weitgehend verloren. ${ }^{430}$

\section{Abbildung 19: Faktionszugehörigkeit der Interviewpartner (LDP)}

\begin{tabular}{l|c|l}
\multicolumn{1}{c|}{ Faktion } & Mitglieder & \multicolumn{1}{c}{ Interviewpartner } \\
\hline Ex-Hashimoto-ha & 51 & Funada, Hori, Ishiba, Kosaka \\
\hline Mori-ha & 51 & - \\
\hline Horiuchi-ha & 33 & - \\
\hline Kamei-ha & 30 & - \\
\hline Yamasaki-ha & 25 & Ôno \\
\hline Kômura (Ex-Kômoto)-ha & 12 & Itô, Usui, Kômura \\
\hline Ozato-ha & 11 & Harada \\
\hline Kôno-Gruppe & 9 & - \\
\hline Nikai-Gruppe & 4 & - \\
\hline Faktionslos & 23 & Hamada
\end{tabular}

Nach: Saitô, Keiko: Seikanyôran, Vol. 22, No. 50/2003, Seisakujihô Ai Pi, Tôkyô 2004, S. 8f. (Saitô, Keiko: Führer durch Politik und Bürokratie.).

Durch die politischen Reformen hat es bei der Kandidatenrekrutierung innerhalb der LDP eine Machtverschiebung von den habatsu hin zur Parteizentrale gegeben. „Durch die Einführung des neuen Wahlsystems ist die Rolle der Parteiführung bei der Kandidatenauswahl gestärkt worden. Von einer Wiederbelebung der früheren Macht der Faktionen kann insgesamt nicht die Rede sein. “431

Christensen kommt zu dem Schluss, dass vor allem die veränderten Finanzströme die Parteizentralen gestärkt haben und der Wert einer offiziellen Kandidatur so gestiegen ist. Die Anerkennung als offizieller Kandidat sei für die Finanzierung des Wahlkampfes wichtiger geworden. ${ }^{432}$ Die Faktionen hingegen sind nicht länger in der Lage, ihre Kandidaten finanziell in dem Maße zu unterstützen, wie es vor den politischen Reformen der Fall war. Damit haben sie zu

\footnotetext{
${ }^{430}$ Vgl.: Krauss, Ellis S.; Pekannen, Robert: Explaining Party Adaption to Electoral Reform: The Discreet Charm of the LDP? A.a.O., S. 14f.

${ }^{431}$ Köllner, Patrick: Drei Jahre nach den politischen Reformen - Eine Zwischenbilanz. Teil 1: Das Wahlgesetz und seine Auswirkungen, a.a.O., S. 175.

${ }^{432}$ Vgl.: Christensen, Raymond V.: The New Japanese Election System, a.a.O., S. 64.
} 
einem großen Teil ihren Einfluss auf die Kandidatenbestimmung in den Einerwahlkreisen verloren. $^{433}$

Ähnlich sahen es auch die befragten Erbabgeordneten der LDP. Der Abgeordnete Itô: „Die Politik der habatsu wie früher ist vorüber, und im Vergleich zur Zeit meines Vaters beträgt die Kraft der habatsu nur noch ein Zehntel. Damals hatten die habatsu alle Macht an sich gerissen, wie etwa bei der Frage der offiziellen Kandidaten, den Wahlkreis eingeschlossen, den Finanzen, darüber hinaus die Rekrutierung für die Parteiämter und die Regierungsposten. Das war, als ob es in der LDP verschiedene Parteien gab. Jetzt haben sie diese Kraft nicht mehr. Sie haben kein Geld mehr. Also vielleicht bezieht sich das jetzt nur auf meine habatsu, aber sie haben kein Geld. Man kann zwar nicht sagen, dass sie keinen Einfluss mehr auf die Rekrutierung haben, aber es ist nicht mehr die Macht wie bei den früheren habatsu. “

Ein Abgeordneter, Usui Hideo, äußerte allerdings die Meinung, die LDP wäre immer noch eine Partei, die sich vor allem aus den habatsu zusammensetzt. Diese hätten weit mehr Einfluss als die Parteizentrale. Interessanterweise gehört Usui derselben habatsu an wie Itô Shintarô. Bei der Frage nach der politischen Rekrutierung machte er allerdings eine Einschränkung: „Es gibt Fälle, in denen die habatsu auf eine Nachfolge hinarbeitet. Aber nicht zwangsläufig muss der Kandidat in der gleichen hababtsu sein. [Der Wahlkreis - d.A.] Chiba-Chûô-ku ist so ein Beispiel, wo der Nachfolger nicht in der selben habatsu ist wie sein Vorgänger. “

Es scheint also nicht so zu sein, dass die Faktionszugehörigkeit gar keinen Einfluss auf eine Kandidatur hat. Nach Klein hat es auch unter dem neuen Wahlsystem Kämpfe wegen der Kandidatenaufstellung zwischen den Faktionen gegeben. Wenn in einem Wahlkreis ein Politiker einer bestimmten Faktion nicht als offizieller Kandidat anerkannt wird, tritt er vielfach als parteiloser Kandidat an und kehrt bei einer erfolgreichen Wahl als Abgeordneter in die Partei zurück. Dabei kann er selbstverständlich auf die Unterstützung seiner Faktion zählen. Insgesamt jedoch haben die Faktionskämpfe abgenommen, da die Kandidaten in ihrem Wahlkreis nicht mehr auf Konkurrenten aus einer anderen Faktion treffen. 434

Der relative Machtverlust der Faktionen hat umgekehrt zu einer Stärkung der Parteiführungen beigetragen. Die Mehrheit der LDP-Abgeordneten sprach von Zentralisierungstendenzen auf Kosten der habatsu als Folge der politischen Reformen. Als Grund für den Machtzuwachs wurde neben dem neuen Wahlsystem vor allem die Politikfinanzierung genannt: „Unter dem Einerwahlsystem tritt nur noch ein Kandidat von der LDP, ein Kandidat von der DPJ usw. an. Damit ist der Einfluss der Partei größer geworden. Wer als Kandidat antritt, entscheidet die

\footnotetext{
${ }^{433}$ Vgl.: Krauss, Ellis S., Pekkanen, Robert: A.a.O., S. 15.

${ }^{434}$ Vgl.: Klein, Axel: A.a.O., S. 211, 259.
} 
Parteizentrale. Dann wird aus der Parteizentrale Geld, also ein Budget für den Wahlkampf und die Aktivitäten, zur Verfügung gestellt. Dass heißt, dass man nicht Sachen sagen darf, die von der Meinung der Parteizentrale abweichen. Solche Anordnungen gibt es. Die Macht der Partei, also der Parteizentrale, ist größer geworden. Sowohl bei der LDP als auch bei der DPJ“, so Funada Hajime.

Während zwei Abgeordnete (Usui, Ishiba) sagten, die Machtverhältnisse hätten sich kaum verändert, sprach der Abgeordnete Hori von einem Machtzuwachs der lokalen Parteiorganisationen. Das ist insofern interessant, als dass Hori gleichzeitig bemerkte, dass sein Wahlkreis eigentlich noch vom „,alten Stil“ geprägt sei. Der Abgeordnete nannte als Beispiel seine Nachfolge im Wahlkreis. Er könne nur dann jemanden bestimmen, wenn auch die Zusammenarbeit der lokalen Parteiorganisation, v.a. der lokalen Parteifunktionäre, gewährleistet sei. Nach den Aussagen der anderen Interviewpartner scheint jedoch der Machtzuwachs der zentralen Parteiebene allgemeine Tendenz zu sein.

Die Machtverschiebung zugunsten der Parteizentralen bedeutet aber nicht zwangsläufig, dass die Chancen einer Erbfolge sinken. Das Vorhandensein eines einflussreichen Fürsprechers in der Partei kann durchaus von Vorteil sein, da ja die Parteizentrale die offizielle Kandidatur bestätigt. Nur sind es jetzt nicht mehr zwangsläufig die Faktionen oder die Faktionsführer, an die sich die Kandidaten wenden. ${ }^{435}$

Ähnlicher Meinung war auch der befragte Abgeordnete Itô. Ihm zufolge bestehen kaum Chancen für einen „Außenseiter“, als offizieller Kandidat anerkannt zu werden. Wer keine Verbindungen zur Parteiführung vorweisen kann, habe kaum Aussichten auf eine offizielle Anerkennung als Kandidat der Partei. Auch durch den Wegfall vieler Mandate seien die Chancen für „Außenstehende“, als Kandidat aufgestellt zu werden, gesunken. Itô Shintarô: „Ob man als offizieller Kandidat der LDP antritt oder nicht, hängt von der Parteizentrale ab, mehr als vom Einfluss der habatsu. In diesem Fall ist das Kriterium für die Entscheidung, wer als Kandidat antritt, dass man der Sohn eines Abgeordneten ist, also Erbabgeordneter, oder vielleicht ein aufgestiegener Regionalparlamentsabgeordnete [...] Die Leute in der Parteizentrale sind jedenfalls schon sehr lange in der Partei und kennen die Väter der Erbpolitiker sehr gut. Zum Beispiel kannten alle Parlamentsabgeordneten meinen Vater. Daher hat man es einfacher, vielleicht aus Mitleid, wenn jemand sagt: ,Das ist Itôs Sohn... '“

\subsubsection{Die Rolle der kôenkai im Rekrutierungsprozess}

Während also für die Anerkennung als offizieller Kandidat die Parteizentrale zunehmend an Einfluss gewinnt, scheinen die kôenkai in den meisten Wahlkreisen immer noch eine wichtige

\footnotetext{
${ }^{435}$ Vgl.: Park, Cheol-Hee: A.a.O., S. 63.
} 
Rolle als Selektionsorgan zu spielen. ${ }^{436}$ Die lokalen Parteiorganisationen konnten sich, wie bereits erwähnt, in der LDP noch nicht als Selektionsorgane durchsetzen. Das liegt vor allem daran, dass die lokalen Parteiorganisationen noch schwach ausgeprägt sind. Kôenkai und Ortsgruppen der LDP existieren vielerorts nebeneinander, die Kandidaten verlassen sich daher lieber auf ihre eigenen Unterstützerorganisationen. In anderen Fällen wurden die Organisationen miteinander verschmolzen und die bisherigen LDP-Kandidaten bzw. -Abgeordneten sind Vorsitzende der lokalen Parteiorganisationen. In diesen Fällen ist es nur schwer vorstellbar, dass selbst bei einer Vorwahl „Außenseiter“ die Chance zu einer Kandidatur bekommen.

Allerdings sprachen einige Abgeordnete bei der Kandidatenrekrutierung von leichten Machtverlusten für die kôenkai. Gegenüber den Parteizentralen hat ihr Einfluss leicht abgenommen. Der Abgeordnete Funada sagte dazu: „Nehmen wir einmal an, ich würde bei der nächsten Wahl verlieren, aber sagen ,ich versuche es noch einmal' und die kôenkai würde auch so denken. Die Parteizentrale könnte dann sagen: ,Wahrscheinlich ist Dein Gegner doch stärker als Du, wenn Du kandidierst. Deshalb tritt jemand anders an.' Solche Fälle gibt es. In letzter Zeit hat es in anderen Wahlkreisen einige solcher Fälle gegeben. Kurz gesagt ist die Macht der Parteizentrale bei der Auswahl der Kandidaten größer geworden. Der Einfluss der einzelnen kôenkai im Wahlkreis ist schwächer geworden. “

Von solchen Fällen berichteten auch zwei weitere LDP-Abgeordnete. Die anderen Abgeordneten der Liberaldemokraten konnten keine Veränderungen feststellen. Der Einfluss der kôenkai scheint also durchaus noch vorhanden zu sein. Lediglich in einigen strittigen Fällen hat sich die Parteizentrale über die Wünsche der kôenkai hinweggesetzt.

\subsubsection{Die key actors in den Oppositionsparteien}

Die Unterschiede bei der Kandidatenaufstellung in den anderen Parteien sind mitunter beträchtlich. Lässt man aber Parteien wie die KPJ, Kômeitô und SDPJ einmal außer acht, kann man feststellen, dass sich die Kandidatenrekrutierung der anderen konservativen Parteien während der Übergangszeit nicht groß von der LDP unterschied. Dennoch führte die staatliche Politikfinanzierung gerade in kleineren Parteien zu einer Stärkung der zentralen Ebene. Zum Beispiel war die Machtkonzentration in der Shinshintô derart groß, dass es zu Auseinandersetzungen zwischen Politikern und den Parteiführern kam, da letztere in alter habatsu-Manier „Taschengelder“ und Posten verteilten. ${ }^{437}$ Sowohl der LDP-Abgeordnete Usui als auch der DPJAbgeordnete Watanabe bestätigten, dass in kleineren Parteien häufig der „Boss an der Spitze“ entscheidet.

\footnotetext{
${ }^{436}$ Vgl.: Köllner, Patrick: Informelle Elemente in der japanischen Politik. Teil 2: Unterstützervereinigungen und Erbabgeordnete, in: Japan aktuell. Wirtschaft. Politik. Gesellschaft, Heft April/1999, S. 172.

${ }^{437}$ Vgl.: Köllner, Patrick: Drei Jahre nach den politischen Reformen, a.a.O.,S. 280 ff.
} 
In der DPJ nehmen Auswahlsysteme wie Vorwahlen und „öffentliche Ausschreibungen“ zu. Dennoch hängt bei dieser Art der Kandidatenbestimmung das Ergebnis sehr stark von der Zusammensetzung des Auswahlgremiums ab. Hierzu gab es sehr unterschiedliche Aussagen der DPJ-Abgeordneten, was damit zusammenhängen kann, dass auch hier die Auswahlverfahren noch nicht vereinheitlicht worden sind. Während Matsuno die zentrale Ebene der Partei, ein Gremium bestehend aus Parteivorsitzendem Generalsekretär und den Abgeordneten, als key actors bezeichnete, sah Watanabe Shû eine Tendenz zu Vorwahlen auf lokaler Ebene, bei denen die lokalen Parteimitglieder über den Kandidaten entscheiden. Gleichzeitig merkte er aber an, dass diese Verfahren noch nicht überall die Regel sind.

Vielmehr stimmten die DPJ-Abgeordneten darin überein, dass zur Zeit die endgültige Entscheidung über eine Kandidatur immer noch bei der Parteizentrale liegt. Interessant ist in diesem Zusammenhang die Aussage von Terata Manabu: „[...] Es entscheidet die Parteiorganisation der Präfektur. Dort wird entschieden. Dort wird ein Aufruf gemacht und wenn der Kandidat geeignet ist, wird er bestimmt und die Entscheidung die Zentralpartei weitergegeben, wo man dann sagt: ,All right'. Sollte die Organisation der Präfektur keinen Kandidaten bestimmt haben, gibt es das System, dass die Zentralpartei jemanden bestimmt. Im Japanischen sagt man dazu ,Fallschirm', ,Parachute’. Daher ist die Parteiorganisation in der Präfektur wie eine Filiale der Parteizentrale, nicht wahr. “

Zwar sagten die DPJ-Abgeordneten, dass die Rekrutierungspfade in ihrer Partei nicht so eng seien wie in der LDP. Dennoch sei, gerade bei der Kandidatenbestimmung auf zentraler Ebene, eine Erbfolge durchaus denkbar. Watanabe Shû: „Diesen von Gefühlen beeinflussten Gedanken „Ich bin seinem Vater etwas schuldig' haben alte Politiker, denke ich. Vielleicht wollen Menschen doch zu einem Teil aufgrund ihrer Gefühle jemanden aussuchen, aber das System muss dazu ,No!’ sagen. Wir müssen jetzt so ein System schaffen. Daher wird sich auch die DPJ ändern, denke ich. “

Erwähnenswert ist an dieser Stelle, dass sich auch in der DPJ Faktionen herausgebildet haben, vor allem in Folge der vielen Parteifusionen. So sind zum Beispiel Gruppen ehemaliger DSP- bzw. SPJ-Abgeordneter entstanden, die unter anderem als Ansprechpartner für Interessengruppen dienen oder junge Kandidaten unterstützen. Im Hinblick auf die Kandidatenrekrutierung soll es auch Einflussversuche dieser Faktionen vor allem bei der Besetzung der Parteiliste gegeben haben. ${ }^{438}$

\footnotetext{
${ }^{438}$ Vgl.: Köllner, Patrick: Zwischen Konflikt, Kohäsion und parteiübergreifender Kooperation: Probleme und Perspektiven der Demokratischen Partei Japans, a.a.O., S. 56f.
} 


\subsubsection{Die Aufstellung der Parteilisten}

Vor allem die Aufstellung der Parteilisten scheint die zentralen Ebenen der einzelnen Parteien gestärkt zu haben. Die Parteilisten werden in erster Linie taktisch eingesetzt, wie Park betont. Während in der LDP die sicheren Listenplätze an ältere Politiker vergeben werden, die auf diese Weise ihren Wahlkreis für jüngere Kandidaten freimachen, werden in vielen Oppositionsparteien die Listen so besetzt, dass sie für die jeweiligen Interessengruppen und Verbände attraktiv sind. ${ }^{439}$

An dieser Stelle muss man hinzufügen, dass Abgeordnete, die über ein Direktmandat ins Parlament einziehen, über ein deutlich höheres Ansehen verfügen als Abgeordnete, die „nur“ über die Parteiliste ein Mandat bekommen haben. Dabei herrscht die Meinung vor, dass die Kandidatur auf der Parteiliste einfacher sei, weshalb man vielen älteren Politiker einen guten Listenplatz zugewiesen hat. Viele Kandidaten in den Direktwahlkreisen beschweren sich über mangelnde Unterstützung der Kandidaten auf den Parteilisten während der Wahlkämpfe. Das hat umgekehrt nicht unbedingt zu einem besseren Ansehen der Listenplätze beigetragen. Klein spricht hier von einer Hierarchie, die unter den Unterhausabgeordneten entstanden ist. An erster Stelle stehen die Abgeordneten, die über ein Direktmandat ins Parlament eingezogen sind. An zweiter Stelle folgen die Abgeordneten, die durch eine Doppelkandidatur über die Parteiliste ein Mandat erhalten haben. An letzter Stelle stehen schließlich die Politiker, die ausschließlich auf der Parteiliste kandidiert haben. ${ }^{440}$

Die Parteilisten schienen auch unter den befragten Erbabgeordneten keinen guten Ruf zu haben. Itô Shintarô äußerte sich dazu folgerndermaßen: „[...] Ob der Politiker, der über die Parteiliste ein Mandat erhält, mit seiner Politik den gestellten Erwartungen gerecht wird, ist noch einmal ein anderes Problem. Das heißt die Reihenfolge der Liste wird nicht entsprechend der Meinungen in der Bevölkerung entschieden, sondern entsprechend der Kräfteverhältnisse in der Parteiführung. Weil vielmehr die Entscheidung der Parteiführung wichtiger ist, wird die Meinung der Bevölkerung nicht berücksichtigt. “

Nur in den seltensten Fällen gibt es transparente Kriterien, nach denen Kandidatenaufstellung vorgenommen wird. Den LDP-Richtlinien zufolge werden Parteilisten von der Parteizentrale auf Empfehlung der Wahlausschüsse in den einzelnen „Blocks“ aufgestellt. ${ }^{441}$ Tatsächlich werden Amtsinhaber bei der Besetzung der Parteilisten in den meisten Parteien bevorzugt und erhalten bei einer Doppelkandidatur bessere Plätze als neue Kandidaten. Das oben genannte Beispiel des Tôkyôter Wahlkreises verdeutlicht indes die verschiedenen Interessenlagen und Ein-

\footnotetext{
${ }^{439}$ Vgl.: Park, Cheo-Hee: A.a.O., s. 49ff.

${ }^{440}$ Vgl.: Bruns, Gabriele: A.a.O., S. 240ff.; Klein, Axel: A.a.O., S. $209 f f$.

${ }^{441}$ Vgl.: Jimintô: Senkyôtaiksaku yôkô, a.a.O.
} 
flussmöglichkeiten alt gedienter Parteiführer. Klein nennt außerdem Fälle, bei denen Listenplätze nach der Höhe der eingeworbenen (und an die Partei weitergegebenen) Spenden vergeben wurden. ${ }^{442}$

Allerdings hat der Ausschuss der LDP für Wahlangelegenheiten 2003 einige Vorschläge für die Besetzung der Parteilisten gemacht. Beispielsweise gibt es Pläne, das Höchstalter von Kandidaten auf der Parteiliste auf 75 Jahre zu begrenzen. Doppelkandidaturen sollen so weit wie möglich abgeschafft werden und ein Kandidat höchstens zweimal in Folge auf der Parteiliste antreten dürfen. Bisher scheinen diese Vorschläge besonders wegen der Altersbegrenzung bei älteren Politikern auf Ablehnung gestoßen zu sein. ${ }^{443}$

Eine Erbfolge über die Parteiliste hielten die meisten Interviewpartner für unwahrscheinlich, auch wenn einige anmerkten, dass bestehende Kontakte des Vorgängers bei der Bestimmung der Liste durchaus von Vorteil sein können.

${ }^{442}$ Vgl.: Klein, Axel: A.a.O., S. 258.

${ }^{443}$ Vgl.: Japan aktuell: Japan aktuell: Gerangel in der LDP um Parlamentskandidaturmodi geht weiter, a.a.O., S. 130. 


\subsection{Die Veränderungen auf der Angebotsseite}

Auch auf der Angebotsseite erwartete man infolge der politischen Reformen eine Öffnung der schmalen Rekrutierungspfade. An die Stelle kostenintensiver, personenzentrierter Wahlkämpfe, so die Annahme, würden nun (partei-) politische Auseinandersetzungen zwischen den Kandidaten gefördert werden. Durch die Einführung von Einerwahlkreisen würden die lokalen Parteiorganisationen aufgewertet und Personen ohne eigene kôenkai die Chance geboten werden, als Kandidat anzutreten. Eine persönliche Unterstützerorganisation wäre damit auch in den konservativen Parteien nicht länger die Vorraussetzung für eine Kandidatur. Auf diese Weise könnte einem größeren Personenkreis die Möglichkeit gegeben werden, sich ungeachtet des familiären oder finanziellen Hintergrunds zur Wahl zu stellen.

Schon kurz nach der Verabschiedung der Reformgesetze wurde Kritik am neuen Wahlsystem laut, die bis heute anhält. Park und Masayama beispielsweise betonen in ihren Untersuchungen, dass auch unter dem neuen Wahlsystem das sanban, also eine Unterstützerorganisation, individuelle Finanzkraft und Bekanntheitsgrad, eine große Rolle spielt und die Vorraussetzung für eine Kandidatur in den konservativen Parteien darstellt. ${ }^{444}$

Wie die Veränderungen auf der Supply-Side und die Folgen für die politische Rekrutierung von den Erbabgeordneten selbst eingeschätzt werden, soll im folgenden Kapitel mit Hilfe der geführten Interviews analysiert werden.

\subsubsection{Kôenkai - besser man hat eine}

Bei der Frage nach der Bedeutung der kôenkai für die Kandidaten gab es je nach Parteizugehörigkeit sehr unterschiedliche Einschätzungen der Interviewpartner. Auf die Rolle der kôenkai in den Wahlkämpfen unter dem neuen Wahlsystem wurde bereits eingegangen. Es scheint zumindest auf Seiten der LDP keine grundlegenden Veränderungen bei der Wahlkampfführung in den Einerwahlkreisen gegeben zu haben. Basis der Kandidaten sind in den meisten Fällen immer noch die persönlichen Unterstützerorganisationen, von denen Köllner sagt, sie entsprächen den Bedürfnissen vieler Wähler nach einer personenzentrierten und lokal ausgerichteten Parlamentariertätigkeit: „Trotz der Einführung eines neuen Wahlsystems, das die formalen Voraussetzungen für partei- und programmorientierten politischen Wettbewerb schafft, haben persönliche Unterstützervereinigungen bisher offenbar nichts von ihrem Wert für die Stimmenmobilisierung der einzelnen Parlamentskandidaten und deren Autonomie gegenüber der Parteizent-

\footnotetext{
${ }^{444}$ Vgl.: Park, Cheol-Hee: A.a.O., S. 53; Masayama Mikitaka: Seijika, seitô, in: Hirano, Hiroshi; Kôno, Masaru: Nihon seijiron, Nihon Keizaihyôronsha, Tôkyô 2003, S. 54. (Masayama Mikitaka: Politiker und Parteien, in: Hirano, Hiroshi; Kôno, Masaru: Theorien japanischer Politik.)
} 
rale verloren. “445 Die Wähler haben sich demzufolge an die überkommenen Beziehungs- und Transaktionsinstrumente gewöhnt, die durch die neuen politischen Rahmenbedingungen sogar stabilisiert werden. Darüber hinaus wahren die Kandidaten durch den Unterhalt eigener Organisationen eine gewisse Unabhängigkeit gegenüber der Parteizentrale.

So verwundert es nicht, dass die meisten befragten LDP-Abgeordneten die Übernahme einer Unterstützerorganisation als einen großen Vorteil für Erbpolitiker bezeichneten. Da der in der Regel langwierige und kostenintensive Aufbau einer eigenen Unterstützerorganisation entfällt, haben Kandidaten, die eine Nachfolge antreten, einen Startvorteil und können sich auf die Erledigung anderer notwendiger Aufgaben konzentrieren.

Viele Abgeordnete wiesen darauf hin, dass es einfacher sei, mit Anhängern zusammen zu arbeiten, die einem „verbunden“ sind. Ein Anhänger der den Vater unterstützt habe, werde wohl auch den Sohn unterstützen. Das erleichtert zumindest in der Anfangszeit die Führung einer kôenkai. „Also wenn schon der Vater unterstützt wurde und der Sohn tritt an, hat er es im Wahlkampf einfacher. Wenn man es von den Leuten aus betrachtet, die den Wahlkampf machen, also aus Sicht der Anhänger, sagt man: ,Na ja, weil es sein Sohn ist, geben wir ihm eine Chance'“, so der Abgeordnete Hori.

Diese Vertrautheit mit den Unterstützern erklärt wohl auch den relativ hohen Anteil von ehemaligen Sekretären unter den Unterhausabgeordneten. Deshalb wies der Abgeordnete Itô auch darauf hin, dass sich eine Nachfolge in der kôenkai nicht unbedingt in Form einer Erbfolge vollziehe: „Das heißt also, dass auf jeden Fall der Kandidat unterstützt wird, der die engsten Verbindungen zu dieser Unterstützerorganisation aufweist. Das ist nicht unbedingt der Sohn in der Erbfolge. “ Dieses Zitat unterstreicht noch einmal die Aussagen von Ishibashi und Reed zur Kandidatenauswahl in den kôenkai. ${ }^{446}$

An der Bedeutung der kôenkai vor allem für LDP-Kandidaten hat sich wohl nichts Grundlegendes verändert. Erbabgeordnete sind vor allem aufgrund ihrer geerbten Unterstützerorganisation so stark. Dieses Phänomen des 1955er-Systems, so die interviewten LDP-Abgeordneten, habe sich erhalten. Tatsächlich ließ sich am Wahlkampf des Präfekturabgeordneten Usui Shôichi der Vorteil einer vom Vater übernommenen kôenkai beobachten. Der Abgeordnete entschied sich relativ spontan wenige Monate vor Beginn des Wahlkampfes für eine Kandidatur. Wegen seines familiären Hintergrunds, so Usui Shôichi, sei ihm der Einstieg aber nicht so schwer gefallen.

\footnotetext{
${ }^{445}$ Köllner, Patrick: Informelle Elemente in der japanischen Politik. Teil 2: Unterstützervereinigungen und Erbabgeordnete, a.a.O., S. 172.

${ }^{446}$ Vgl.: Kapitel 2.4.
} 


\subsubsection{DPJ-Kandidaten und Unterstützervereinigungen}

Die befragten Erbabgeordneten der DPJ waren bezüglich der Bedeutung persönlicher Unterstützervereinigungen anderer Meinung. Vor allem Matsuno Yorihisa betonte, er stütze sich im Wahlkampf nicht mehr auf eine kôenkai, sondern auf die lokalen Parteiorganisationen. In seinem Fall hätte dann die Bedeutung der lokalen Parteiorganisation tatsächlich zugenommen und die Reformen hätten den gewünschten Erfolg gehabt.

Die Abgeordneten Watanabe und Terata, die ihren Wahlkampf nach eigenen Angaben auf eigenen Unterstützerorganisationen aufbauen, waren zwar der Meinung, eine starke kôenkai sei durchaus von Vorteil. Vor allem für Kandidaten, die als Unabhängige antreten, seien sie Vorraussetzung für eine Kandidatur. Dennoch würden diese Organisationen keinesfalls eine Garantie mehr für einen Mandatsgewinn darstellen, wie Terata Manabu anmerkte. Der tatsächliche Wert einer eigenen Unterstützerorganisation sei seiner Meinung nach im Vergleich zum alten Wahlsystem weitaus geringer: „Wenn man es sich so überlegt, hat man unter dem aktuellen Wahlsystem Alternativen. Ich glaube, man kann auch gewinnen, wenn man keine kôenkai hat. In der letzten Wahl hatten wir viele Fälle, bei denen Kandidaten, die erst zehn Monate dabei waren, ganz dicht an die Politiker herankamen, die eine in 20 oder 30 Jahren mühsam aufgebaute, bis in die vierte Generation geführte Basis hatten. Die kôenkai sind nach wie vor stark. Besser man hat eine. Verglichen mit früher hat ihre Stärke aber abgenommen. “

Dies gelte, so die Abgeordneten, vor allem für Kandidaten der DPJ. Hier hätten Aspiranten ohne eigene kôenkai weitaus besseren Chance auf eine Kandidatur als in der LDP. Auf diesen Punkt wird später im Zusammenhang mit Problemen bei der Kandidatenrekrutierung noch genauer eingegangen.

\subsubsection{Expansion und Fusion von kôenkai}

Auch wenn die Übernahme einer Unterstützerorganisationen für Erbabgeordnete immer noch einen Vorteil darstellt, scheinen die politischen Reformen an dieser Stelle doch einige Veränderungen bewirkt zu haben. Eine Herausforderung, vor die sich die meisten Kandidaten in der Übergangszeit zum neuen Wahlsystem gestellt sahen, war das Zusammenwachsen mit anderen Organisationen.

Die Netzwerke der Kandidaten mussten neu geordnet werden, was gerade in den konservativen Parteien zu Problemen geführt hat. Da 211 Direktmandate weggefallen sind und nur 180 Listenplätze neu geschaffen wurden, entstanden in vielen Wahlkreisen „herrenlos“ gewordene Unterstützervereinigungen. Diese kôenkai gehörten Abgeordneten oder Kandidaten, die in den neu geschaffenen Einerwahlkreisen keine Aussicht mehr auf eine erfolgreiche Kandidatur hatten. 
Darüber hinaus wurden durch die Neueinteilung der Wahlkreise Netzwerke auseinander gerissen. Umgekehrt waren die Kandidaten damit konfrontiert, dass nun Teile anderer Netzwerke in ihrem Bezirk lagen. Eine Vereinigung mit anderen Gruppen auf dem eigenen Gebiet war also notwendig. Man darf deshalb nicht vergessen, dass diese „herrenlosen“ kôenkai den übrig gebliebenen Kandidaten auf der anderen Seite auch Chancen boten, ihre Basis auszubauen. In vielen Fällen wurden Absprachen mit Kandidaten in anderen Wahlkreisen getroffen, Teile der Organisationen ,auszutauschen“. ${ }^{447}$

Dennoch ergab sich gerade in der LDP das Problem, dass diese herrenlosen kôenkai häufig nicht bereit waren, einen anderen Kandidaten der selben Partei zu unterstützen. Auch wenn die Zusammenarbeit häufig reibungslos funktionierte, gab es viele Fälle, in denen die Zusammenlegung scheiterte. Zwischen den Organisationen verschiedener Kandidaten der gleichen Partei bestanden nicht selten tiefe Feindschaften. ${ }^{448}$ Da die Wahlkämpfe unter dem alten Wahlsystem sehr personalisiert waren, kam es durchaus auch zu scharfen Angriffen auf den Konkurrenten, selbst wenn er aus der eigenen Partei kam. „Weil sie [die Kandidaten einer Partei - d.A.] gegeneinander antreten mussten, waren die Beziehungen unter den LDP-Kandidaten am schlimmsten von allen: ,Es ist unbegreiflich, was er [der andere LDP-Kandidat - d.A.] macht. Er ist ein schlechter Kerl. Ich bin der einzige, der geeignet ist.' So führte man Wahlkampf. Das war wirklich überflüssig, denke ich“, so der Abgeordnete Ishiba.

Unter dem alten Wahlsystem gab es zwar in vielen Fällen Absprachen zwischen einzelnen Kandidaten, die sozusagen ihr „Terrain“ absteckten. Dennoch gibt es genügend Beispiele, wo ein Kandidat versuchte, seine Basis auf Kosten eines Parteikollegen zu vergrößern. Das führte mitunter zu Feindschaften, die mit Einführung des neuen Wahlsystems nicht zwangsläufig beendet waren. Von diesen Problemen wussten auch viele Interviewpartner wie etwa der Abgeordnete Ôno zu berichten: „Als der Wahlkreis zum Einerwahlkreis wurde, gewann ich ihn. Die anderen beiden kôenkai sind aber noch da. Sie sind überhaupt nicht zusammengewachsen. Das ist typisch japanisch. “449

Der Abgeordnete Funada betrachtete die Zusammenlegung der Unterstützerorganisationen in seinem Fall zwar als Erfolg, merkte aber an: „Ich habe nun die Mitglieder der anderen beiden kôenkai gefragt, ob sie nicht in meine Organisation eintreten wollen und sie gebeten, mich zu unterstützen. Und nach und nach traten sie bei mir ein. [...] Nun ist es so, dass es Leute gibt,

\footnotetext{
${ }^{447}$ Vgl.: Christensen, Raymond V.: The Effects of Electoral Reform on Campaign Practices in Japan, a.a.O., S. 986; 992f.

${ }^{448}$ Vgl.: Klein, Axel: A.a.O., S. 225; Köllner, Patrick: Informelle Elemente in der japanischen Politik. Teil 2:

Persönliche Unterstützervereinigungen und Erbabgeordnete, a.a.O., S. 169.

449 Ôno bezieht sich hier auf die bereits beschriebenen Strukturen japanischer Gruppen, die hierarchisch aufgebaut sind und sich sehr stark nach außen hin abgrenzen. Eine Vereinigung von Gruppen ist daher relativ schwierig.
} 
die man mag, und welche, die man nicht mag. Leute, die vorher für einen anderen Abgeordneten von der LDP gearbeitet haben, unterstützen jetzt mich. Das heißt also - man kann es sich natürlich denken - dass es Leute gibt, die in Wirklichkeit das Gefühl haben: ,Na ja, den Kandidaten möchte ich ja eigentlich doch nicht unterstützen.' Von denen gibt es nicht viele, nur einige wenige. Aber dass solche Leute unter meinen Anhängern sind, ist doch ein großes Problem."

Das wirkt sich auch auf die genannten Fusionen der kôenkai mit den lokalen Parteiorganisationen aus, wie der Abgeordnete Ôno feststellen musste: „Die Parteimitglieder und meine kôenkai sind zusammengewachsen, aber unter den Parteimitgliedern sind oft welche, die einen anderen Kandidaten unterstützen. Dieses Problem müsste man öffentlich diskutieren. “

Nach Kataoka gab es sogar Fälle, in denen sich Parteimitglieder aus Protest gegen eine Nachfolgeregelung einer anderen politischen Gruppierung anschlossen. ${ }^{450}$ Aus diesen Gründen verlassen sich Kandidaten wohl lieber auf Unterstützervereinigungen, die an ihre Person gebunden sind. Christensen merkt in diesem Zusammenhang an, dass die erfolgreiche Fusion verschiedener Gruppen vor allem von der Stärke des Kandidaten abhängt. Als Erklärung wird ein so genannter bandwagon-Effekt angeführt: Die Menschen laufen, da sie auf der Seite des Siegers stehen wollen, dem Wagen mit der „Musikkapelle“ nach. Zwar gibt es in den herrenlos gewordenen Organisationen durchaus Mitglieder, die vielleicht sogar aus ideologischen Gründen nicht für den neuen Kandidaten arbeiten wollen. Letztendlich werden erfolgreiche Kandidaten aber wegen ihres Zugangs zur nationalen Politik doch unterstützt. ${ }^{451}$

Ein weiteres Problem für die Kandidaten stellt in den neu geschaffenen Einerwahlkreisen die Tatsache dar, dass nun in der Regel eine größere Stimmenzahl notwendig ist, um ein Mandat zu gewinnen. Das bedeutet, dass sich die Politiker, die ja jetzt um die Stimmenmehrheit in ihrem Wahlkreis kämpfen, nicht mehr allein auf die effektive Unterstützung ihrer kôenkai verlassen können. Sie müssen auch die Wählerschaft außerhalb ihrer Netzwerke ansprechen. ${ }^{452}$

Der Abgeordnete Funada dazu: „Zur Zeit der Mehrerwahlkreise reichte es, wenn man von den Wählern ein Viertel, na ja sagen wir ein Fünftel zusammenbekam. Wenn allein die mich wählten, konnte ich gewinnen. Wenn mich jetzt im Einerwahlkreis nicht die Hälfte, ja wenn möglich mehr als die Hälfte der Wähler, unterstützt, kann ich nicht gewinnen. Kurz gesagt reicht meine kôenkai, meine bisherige Organisation, allein nicht mehr aus. “

\footnotetext{
${ }^{450}$ Vgl.: Kataoka, Masaaki: Changing local Politics: Party Realignment and growing Competition, a.a.O., S. 209.

${ }^{451}$ Vgl.: Christensen, Raymond V.: The Effects of Electoral Reform on Campaign Practices in Japan, a.a.O., S. 993.

${ }^{452}$ Vgl.: Bruns, Gabriele: A.a.O., S. 244; Klein, Axel: A.a.O., S. $224 f f$.
} 
Die Kandidaten stehen heute vor der Herausforderung, mit ihrer Wahlkampforganisation mehr Wähler ansprechen zu müssen. Der befragte Abgeordnete Kosaka gab an, dass er früher 60\% der benötigten Stimmenzahl über seine kôenkai gewonnen habe. Jetzt liege diese Zahl nur noch bei 30\%, in den Städten sogar darunter. Seine Unterstützerorganisation reiche daher längst nicht mehr aus, um die Wahl für sich zu entscheiden.

Zwar sind die Wahlkreise kleiner geworden und das Ergebnis hängt von der Zahl der relevanten Kandidaten ab, dennoch ist die erforderliche Stimmenzahl für einen Mandatsgewinn wohl allgemein gestiegen. Viele Kandidaten sind deshalb dazu übergegangen, neben den genannten herrenlosen Organisationen andere Verbände, wie etwa Zahnärzteverbände, noch stärker in ihre Organisation einzubinden. ${ }^{453}$

Köllner führt in diesem Zusammenhang eine zunehmend bessere Zusammenarbeit zwischen Lokalpolitikern und den Kandidaten für das Unterhaus an. Da es in einem Wahlkreis nur noch einen Kandidaten einer Partei gibt, müssen sich die Lokalpolitiker in den Unterhauswahlen auf diesen festlegen. ${ }^{454}$ In den Mehrerwahlkreisen gab es schließlich mehrere Kandidaten einer Partei, so dass es je nach Interessenlage auch häufig wechselnde Koalitionen von Unterstützervereinigungen gab. In diesem Sinne hatten die politischen Reformen sicherlich einen positiven Effekt.

Allerdings mussten gerade in der Übergangszeit die zwischen den Abgeordneten auf nationaler Ebene und den Lokalpolitikern bestehenden Netzwerke neu geordnet werden. Das traf vor allem auf Politiker zu, die sich von der LDP abgespalten hatten und nun in die neuen konservativen Parteien übertraten. Die Lokalpolitiker standen somit vor dem Dilemma, entweder jahrelang aufgebaute Beziehungen aufzugeben oder aber dem Politiker in eine Partei zu folgen, die eventuell nicht über Ressourcen in einem Ausmaß wie die LDP verfügte. Nach Kataoka hatte die LDP häufig auf regionaler Ebene sehr großen Einfluss in den Präfekturparlamenten. Folgt der Lokalpolitiker seinem „Patron“ in eine andere Partei, bedeutet das natürlich auch einen gewissen Einflussverlust im Parlament für ihn. Einige Präfekturabgeordnete zögerten deshalb möglichst lange, eine klare Entscheidung hinaus und versuchten trotz Zugehörigkeit zur LDP die bereits bestehenden Loyalitätsverhältnisse zu „ihren“ Unterhausabgeordneten aufrecht zu erhalten. Hinzu kam, wie bereits erwähnt, dass durch die Neuordnung der Wahlkreise Loyalitätsverhältnisse zwischen Lokalpolitikern und den Kandidaten für das Unterhaus beendet beziehungsweise neu aufgebaut werden mussten. ${ }^{455}$

\footnotetext{
${ }^{453}$ Vgl.: Park, Cheol-Hee: A.a.O., S. $75 f$.

${ }^{454}$ Vgl.: Köllner, Patrick: Informelle Elemente in der japanischen Politik. Teil 2: Persönliche Unterstützervereinigungen und Erbabgeordnete, a.a.O., S. 169.

455 Vgl.: Vgl.: Kataoka, Masaaki: Changing local Politics: Party Realignment and growing Competition, a.a.O., S. 208ff.
} 
Die Zusammenarbeit zwischen offiziellen Parteiorganisationen und persönlichen Unterstützerorganisationen scheint sich ebenfalls leicht verbessert zu haben. Zwar stellen die kôenkai in der Regel weiterhin die Basis der Abgeordneten dar, in der LDP wird aber auf eine bessere Zusammenarbeit zwischen den kôenkai und den lokalen Parteiorganisationen gedrängt. ${ }^{456} \mathrm{Al}-$ lerdings gelten die kôenkai immer noch als eine Art Auffangbecken für Anhänger, die zwar den Kandidaten unterstützen, der Partei aber sehr kritisch gegenüber stehen. In einigen kôenkai finden sich sogar die Mitglieder unterschiedlicher Parteien. Gerade in Wahlkreisen, wo eine Partei nicht sehr beliebt ist, scheint die Wahrscheinlichkeit deshalb gering, dass ein Kandidat dieser Partei seine kôenkai mit der lokalen Parteiorganisation zusammenlegt. ${ }^{457}$

Auf einen weiteren interessanten Aspekt wies der Abgeordnete Funada hin. Da die Anhänger in seiner kôenkai, die bereits seinen Großvater unterstützt haben, mitunter schon 80 oder 90 Jahre alt sind, steht er vor dem Problem, seine Organisation verjüngen zu müssen. Dies sei aber eine Aufgabe, vor der auch andere Kandidaten stehen, wenn sie bereits über mehrere Wiederwahlraten verfügen. In diesem Sinn stellt die Übernahme einer kôenkai für Erbpolitiker tatsächlich eher einen Vorteil zu Beginn der politischen Karriere dar. Im Laufe der Abgeordnetentätigkeit sind die Herausforderungen, die die Führung einer kôenkai darstellt, bei Erbabgeordneten und Nichtabgeordneten die gleichen.

Zusammenfassend lässt sich sagen, dass für Erbpolitiker offensichtlich immer noch Vorteile bei Übernahme einer kôenkai bestehen, auch wenn es mitunter große Unterschiede zwischen Stadt und Land zu geben scheint. Je nach Parteizugehörigkeit lassen sich jedoch einige Unterschiede in den Antworten der Befragten feststellen. So ist in der DPJ nach Aussage ihrer Abgeordneten eine eigene kôenkai als Vorraussetzung für eine Kandidatur nicht mehr unbedingt notwendig.

Japanische Wahlkämpfe sind, wie bereits erläutert wurde, immer noch sehr stark auf die Person des Kandidaten und auf die Pflege zwischenmenschlicher Beziehungen ausgerichtet. Die lokalen Parteiorganisationen scheinen zumindest in der LDP noch keine Alternative zum Unterhalt einer persönlichen Unterstützerorganisation zu bieten. Auch Fusionen dieser beiden Organisationstypen sind nicht unproblematisch. ${ }^{458}$ Die kôenkai wurden vielmehr den Erfordernissen der neuen politischen Rahmenbedingungen angepasst und bilden weiterhin die Basis der Kandidaten: „Existing organizations are as likely to be adapted to somewhat different functi-

\footnotetext{
${ }^{456}$ Vgl.: Jiyûminshutô: Senkyotaisaku yôkô, a.a.O.

${ }^{457}$ Vgl.: Krauss, Ellis S.; Pekannen, Robert: Explaining Party Adaption to Electoral Reform: The Discreet Charm of the LDP? A.a.O., S. 11f.

${ }^{458}$ Vgl.: Vgl.: Krauss, Ellis S.; Pekannen, Robert: Explaining Party Adaption to Electoral Reform: The Discreet Charm of the LDP? A.a.O., S. 11.
} 
ons in a new electoral system, as old organizational forms are to be scrapped and totally new ones created. Changed systems and incentives do not necessarily require replacement of organizations, merely their adaption." 459

Allerdings wiegen die Vorteile einer kôenkai-Übernahme nach Aussage der meisten Interviewpartner nicht mehr so schwer wie unter dem alten Wahlsystem. Zwar waren sich die LDPAbgeordneten einig, dass die persönlichen Unterstützerorganisationen nach wie vor die Basis zur Stimmengewinnung darstellen, sie allein reichen jedoch für einen Mandatsgewinn nicht mehr aus. Die dadurch erforderliche Fusion und Expansion der Organisationen hat auch zu einer Heterogenisierung der kôenkai geführt und einige Probleme mit sich gebracht. ${ }^{460}$

\subsubsection{Die Tasche - immer schwerer zu füllen}

Stimmten die Aussagen der befragten Abgeordneten bei der Frage nach den Vorteilen bei der Übernahme einer kôenkai zum größten Teil überein, wurde der finanzielle Aspekt einer Erbfolge sehr unterschiedlich eingeschätzt. Vor allem unter den LDP-Abgeordneten fanden sich viele Interviewpartner, die sagten, dass die finanziellen Vorteile für Erbabgeordnete abgenommen hätten. Das wurde zumeist in einen direkten Zusammenhang mit den politischen Reformen gestellt. Die „Tasche“ sei nun immer schwerer zu füllen. Vor allem das bereits erwähnte veränderte Spendenverhalten scheint dazu geführt zu haben, dass allgemein die Spendeneinkünfte japanischer Abgeordneter gesunken sind und daher Erbabgeordneten auch weniger Vorteile bieten. Dafür ist der Anteil aus den Erlösen von „Fund-Raising-Partys“ beträchtlich gestiegen. ${ }^{461}$ Es ist also denkbar, dass Unternehmensspenden nun über diesen Umweg an die Kandidaten gelangen.

Tatsächlich sanken offiziell die Einnahmen der Abgeordneten 2004 auf einen neuen Tiefstand. Gegenüber 2003 nahmen die Parlamentarier etwa 8\% weniger ein. Das Durchschnittseinkommen japanischer Unterhausabgeordneter liegt derzeit bei etwa 26,5 Mio. Yen. Drei Abgeordnete nahmen mehr als 100 Mio. Yen ein, der LDP-Abgeordnete Asô Tarô sogar 720 Mio. Yen aufgrund von Immobiliengeschäften. ${ }^{462}$

Auch das Durchschnittsvermögen der Abgeordneten ist gesunken und liegt im Schnitt bei 51,6 Mio. Yen. Im Jahr 2000 lag die Zahl noch 15,6 Mio. Yen höher. Diese Angaben berücksichtigen allerdings nicht das Aktienvermögen der Abgeordneten. Vor allem neu gewählte Abgeordnete scheinen jedoch zumeist über ein geringes Vermögen zu verfügen. 30 Abgeordnete

\footnotetext{
${ }^{459}$ Vgl.: Ebd., S. 28.

${ }^{460}$ Vgl.: Ebd., S. 12.

${ }^{461}$ Vgl.: Köllner, Patrick: Parteienfinanzierung in Japan: Regulierungsmechanismen und Anpassungsstrategien, a.a.O., S. 154.

${ }^{462}$ Vgl.: Japan aktuell: Einkommen von Parlamentariern sinkt 2003 auf neuen Tiefstand, in: Japan aktuell. Wirtschaft. Politik. Gesellschaft, Heft August/2004, S. 289.
} 
waren sogar mit mehr als 100 Mio. Yen verschuldet. Reichster Abgeordneter Japans ist Hatoyama Yukio mit 1,77 Mrd. Yen. ${ }^{463}$

Bei diesen Zahlen handelt es sich allerdings um offizielle Angaben des japanischen Unterhauses. Die tatsächlichen Einnahmen können wesentlich höher sein, da viele Abgeordnete, wie bereits beschrieben, lokale Parteiorganisationen als zusätzliche Geldbörse nutzen. Hinzu kommt, dass falsche Angaben der Abgeordneten nicht bestraft werden. ${ }^{464}$ Es ist also gut möglich, dass die Einnahmen japanischer Abgeordneter nicht in dem genannten Maß gesunken sind.

Die Ausgaben japanischer Abgeordneter scheinen nämlich nach wie vor recht hoch zu sein. Abgesehen von drei Sekretären im Parlament, die staatlich finanziert werden, hat ein Abgeordneter durchschnittlich noch weitere fünf Sekretäre in den Wahlkreisen selbst. Die Zahl der privaten Sekretäre unterscheidet sich je nach Partei. Während ein KPJ-Abgeordnete im Schnitt nur einen Sekretär zusätzlich unterhält, kommt ein LDP-Abgeordneter auf fast sieben Sekretäre. Das bedeutet jährliche Kosten von etwa 23,3 Mio. Yen. Ebenfalls müssen die Miete für die entsprechenden Wahlkreisbüros und das Material bezahlt werden, zusammen weitere 20 Mio. Yen. Die Kosten für den Unterhalt der kôenkai, Werbung etc. können nur geschätzt werden, Masayama kommt aber auf einen Finanzbedarf von 100 bis 150 Mio. Yen, den ein japanischer Abgeordneter jährlich hat. Der finanzielle Aspekt bei einer Kandidatur sollte also nicht unterschätzt werden. ${ }^{465}$

Zwei Interviewpartner gaben allerdings an, dass sie ihre Wahlkampfausgaben verringert hätten. Dazu trügen neben den verringerten Einnahmen vor allem die neuen Wahlkampfbestimmungen bei, wie etwa Vorschriften für die Zahlung von Aufwandsentschädigungen und die verschärften Sanktionen bei Gesetzesüberschreitungen. Hinzu kommt das Spendenverhalten der Unternehmen, die zum Einen nicht mehr an Einzelpolitiker spenden dürfen und zum Zweiten wegen der zurückliegenden Skandale vorsichtiger geworden sind.

Andere Abgeordnete wie Itô Shintarô hingegen kritisierten, dass „Außenseiter“ finanziell immer noch wenig Chancen auf eine Kandidatur haben: „Ich bin nun Erbabgeordneter, aber normale Leute, die keine Erbabgeordneten sind, die über keine Kontakte zur Parteizentrale verfügen, sind vielleicht von ihrer Einstellung her der LDP sehr nah, möchten starten, haben aber nicht die finanziellen Möglichkeiten. “

\footnotetext{
${ }^{463}$ Vgl.. Japan aktuell: Durchschnittsvermögen von Unterhausabgeordneten niedriger als zuvor, in: Japan aktuell. Wirtschaft. Politik. Gesellschaft, Heft Juni/2004, S. 213.

464 Vgl.: Japan Times: Lawmakers' average income drops to new low, in: The Japan Times Online, http://search.japantimes.co.jp/print/news/nn07-2005/nn20050705a5.htm, Download vom 12.07.2005.

${ }^{465}$ Vgl.: Masayama, Mikitaka, a.a.O., S. 54-55.
} 
Masayama sieht immer noch einen direkten Zusammenhang zwischen der Politikfinanzierung und der nach wie vor hohen Zahl von Erbabgeordneten. Die (LDP-) Abgeordneten vergleicht er mit mittelständischen Unternehmern, die sich mit dem Wechsel in die Politik selbstständig machen, aber auch dem Risiko ausgesetzt sind, bei einer Wahlniederlage bankrott zu gehen. So erklärt Masayama, dass sich der Generationswechsel in der japanischen Politik in erster Linie durch Nachfolgeregelungen vollzieht und die Zahl der Erbabgeordneten, aber auch die der ehemaligen Sekretäre und Regionalpolitiker, so hoch ist. ${ }^{466}$

Viele Abgeordnete äußerten allerdings die Meinung, dass die Folgen der neuen Bestimmungen zur politischen Rekrutierung noch nicht im ganzen Umfang abzusehen sind. Auch wenn es noch gewisse finanzielle Vorteile für Erbabgeordnete gibt, scheinen sie im Verhältnis zum früheren Wahlsystem abgenommen zu haben.

Einig waren sich die Interviewpartner lediglich darüber, dass die finanzielle Situation den Machtzuwachs der Parteizentralen verstärkt hat. Nicht nur in kleineren Parteien, sondern auch in der LDP oder DPJ, hat die staatliche Finanzierung zu einer Stärkung der zentralen Ebene geführt. Der Abgeordnete Kosaka dazu: „Ein anderer Punkt ist, dass ja früher die habatsu Geld verteilten, nicht wahr. Damit unterstützten sie die Kandidaten. Jetzt müssen die Kandidaten ihr eigenes Geld verwenden. Aber weil sie nicht so viel eigenes Geld ausgeben können, steigt die Abhängigkeit von der Partei, also den Wahlkampfhilfen oder den Budgets der Partei. Ich glaube, dass es einen Wandel von der Abhängigkeit von den habatsu zu einer Abhängigkeit von der Partei gegeben hat. “

Diese Machtverschiebung bleibt jedoch nicht immer konfliktfrei. In der früheren Shinshinto war die Machtkonzentration derart groß, dass es zu Auseinandersetzungen zwischen den Abgeordneten und den Parteiführern kam, da letztere in alter habatsu-Manier „Taschengelder“ und Posten verteilten. ${ }^{467}$

\subsubsection{Mitleidstimmen und ein bekannter Name}

Eine sehr viel größere Bedeutung kommt den Aussagen der Interviewpartner zufolge dem bekannten Namen, dem kanban, zu. Alle befragten Abgeordneten waren der Meinung, dass der bekannte Name den größten Vorteil für Erbabgeordnete darstelt. Das gilt sowohl für die befragten LDP-Abgeordneten als auch für die Parlamentarier der DPJ.

Köllner weist darauf hin, dass gerade bei den Kandidaten der konservativen Parteien politische Unterschiede häufig nur sehr schwer festzustellen sind und eine parteiprogrammatische

\footnotetext{
${ }^{466}$ Vgl.: Masayama, Mikitaka, a.a.O., S. 55-56.

${ }^{467}$ Vgl.: Köllner, Patrick: Drei Jahre nach den politischen Reformen - Eine Zwischenbilanz Teil II: Die Finanzierung politischer Aktivitäten, a.a.O., S. 280 ff.
} 
Wahl sehr schwierig machen. ${ }^{468}$ In diesem Fall sind der Ruf und der Bekanntheitsgrad eines Kandidaten also nach wie vor von großer Bedeutung. Wie bereits gezeigt wurde, haben sich die Wahlkampfstile und -methoden kaum verändert. Einige Abgeordnete sprachen davon, dass die in vielen Jahren beim Wähler entstandene Vertrautheit mit dem Familiennamen zur Abgabe einer „emotionalen“ Stimme führt.

Der Abgeordnete Ishiba betrachtete den bekannten Namen sogar als einen geldwerten Vorteil, über den ein Erbpolitiker verfügt: „Auch nach Ende des 1955er-Systems gibt es viele Erbabgeordnete. Sowohl in der LDP als auch in der DPJ. Einfach ausgedrückt: Wenn sie zur Wahl antreten, wird ihr Name verkauft. Diese Leute lassen die Wähler denken, dass ihre Kinder genauso gut sind wie sie. Das heißt, es kostet nichts. Das war unter dem 1955er-System so, und das ist jetzt, nach dem Ende des 1955er-Systems, immer noch so. “

Eng mit dem bekannten Namen sind so genannte Mitleidstimmen verknüpft, deren Bedeutung vor allem in ländlichen Wahlkreisen nach wie vor recht groß ist, wie der Abgeordnete Hori anmerkte: „Wenn der Vater stirbt, wird auch Mitgefühl geweckt. Deshalb treten Politiker in der zweiten und dritten Generation unter einfacheren Bedingungen an. Besonders in den ländlichen Gebieten Japans kommt das sehr häufig vor. “

Der Abgeordnete Hori wies in diesem Zusammenhang auf das so genannte ihai hin. Dabei handelt es sich um buddhistische Totentafeln, auf die der buddhistische Name des Verstorbenen geschrieben wird, den er nach seinem Tod von einem Tempel erhält. Sie dienen dem Gedenken der Hinterbliebenen. Ein Erbfolger besitzt im gewissen Sinn die Totentafel seines Vorgängers und kann damit die genannten Mitleidstimmen mobilisieren. Die Wähler unterstützen im Wahlkampf nun den Sohn, dessen Vater sie für seine erfolgreiche Wahlkreisarbeit dankbar sind. Der Befragte sprach hier aus eigener Erfahrung. Dieses ihai stelle zumindest in der Anfangsphase einen Vorteil für Erbabgeordnete dar, die dann allerdings auch vor der Aufgabe stünden, in die (noch recht großen) Fußstapfen des Vaters zu treten.

Allerdings gab der Interviewpartner zu bedenken, dass dieser Vorteil vor allem in den Städten langsam verloren geht. Eine Verallgemeinerung sei daher sehr schwierig. Der Abgeordnete Watanabe war ähnlicher Ansicht: „In meinem Bezirk ist es auch so, weil dort neue Leute hinziehen und sich die Bevölkerungsstruktur verändert. Weil diese Leute den Namen meines Vaters oder seine früheren Errungenschaften für den Wahlkreis nicht kennen, empfinden sie auch keine Dankbarkeit. Auf diese Weise ändert sich etwas, denke ich. “

Doch selbst wenn die Wählerschaft keine Dankbarkeit gegenüber dem Vorgänger empfindet, dient der Name zumindest dazu, ins Gespräch zu kommen. Dabei kann durchaus auch ein

\footnotetext{
${ }^{468}$ Vgl.: Köllner, Patrick: Drei Jahre nach den politischen Reformen - Eine Zwischenbilanz Teil I: Das Wahlgesetz und seine Auswirkungen, a.a.O., S. 173.
} 
schlechter Ruf von Nutzen sein. „Im Allgemeinen gelten Erbabgeordnete von Natur aus als schlecht. Sie haben also nicht nur Vorteile. Aber es ist wohl besser, man ist berühmt, berüchtigt, ,notorious' [der Abgeordnete benutzt hier das englische Wort - d.A.], und hat einen Ruf. Der Bekanntheitsgrad ist ein sehr großer Vorteil. Ich bin auch der Sohn meines Vaters und war zu Anfang berüchtigt, ,notorious', nicht wahr. Aufgrund des Namens kann ich die Leute aber einfacher ansprechen. Wenn ich sie anspreche, bewerten sie mich neu und ich habe nicht mehr so einen schlechten Ruf, sondern sie sehen meine wahren Beweggründe. Ich sehe es vielleicht gerade durch eine gefärbte Brille, aber die Wähler hören niemandem zu, von dem sie nicht wissen, wer man ist. Es ist ein großer Vorteil, jemanden anzusprechen, wenn die Leute wissen, wer man ist. Egal, ob man ein guter oder ein schlechter Mensch ist. “

In diesem Zusammenhang werden immer wieder die sehr strengen Wahlkampfbestimmungen genannt. Da Wahlkampfwerbung sehr stark reglementiert ist, haben es „neue Gesichter“ schwer, sich zu etablieren. Es fällt ihnen sehr schwer, überhaupt ins Gespräch zu kommen. ${ }^{469}$

Der bekannte Name, bzw. in diesem Fall der Familienname, stellt also für Erbpolitiker neben der Übernahme einer kôenkai den größten Vorteil dar. Dies gilt zwar nur eingeschränkt für die Großstädte, in denen die Bevölkerung recht häufig fluktuiert und sich die genannten Abhängigkeits- und Loyalitätsverhältnisse nicht so leicht erhalten lassen. Zumindest auf dem Land hat es ein Erbfolger jedoch einfacher, mit den Wählern ins Gespräch zu kommen und eventuell einige Stimmen aus Dankbarkeit gegenüber dem Vorgänger oder sogar aus Mitleid zu gewinnen. Dieser bekannte Name stellt auf diese Weise gleichzeitig einen gewissen finanziellen Vorteil dar.

\subsubsection{Der Junge vor dem Tempeltor - Das Hineinwachsen in einen Beruf}

Ein weiterer Aspekt, der in den Fragebögen 2003 noch nicht berücksichtigt worden war, von fast allen Interviewpartner aber angesprochen wurde, war das „Hineinwachsen in die Politik“. Bei der Befragung 2005 wurde dieser Aspekt zwar nicht als gesonderter Fragekomplex in den Fragebogen aufgenommen, da er sich aus den Fragen nach dem eigenen Einstieg und den Anreizen bzw. Zwängen einer Erbfolge ergab. Dieser Punkt stellte aber aufgrund seiner offensichtlichen Bedeutung für den Rekrutierungspfad des Erbabgeordneten später eine zusätliche Auswertungskategorie dar. Ähnlich wie beim bekannten Namen, ließen sich auch hier keine Unterschiede nach Parteizugehörigkeit feststellen.

Einige Abgeordnete nannten in den Interviews ein bekanntes japanisches Sprichwort, das besagt, dass ein Junge vor dem Tempeltor die Sutren („Lehrreden“ oder Leitfaden des Buddhis-

${ }^{469}$ Vgl.: Hrebenar, Ronald J.: Rules of the Game: The Impact of the Electoral System on Political Parties, a.a.O., S. 53. 
mus) lesen kann, ohne dafür besonders ausgebildet zu sein. Damit ist gemeint, dass das Aufwachsen in einer Politikerfamilie zu einer gewissen Vertrautheit mit der Arbeit eines Politikers führt. Bestimmte Verhaltensweisen werden von Kindheit an unbewusst antrainiert.

Der Abgeordnete Itô dazu: „[...] Als erstes fällt mir die Erziehung durch den ,Platz vor dem Tempeltor' ein. Weil die Kinder von Abgeordneten seit dem Kindergarten ihre Eltern als Politiker erleben, lernen sie, was ein Politiker ist. Ohne in eine Schule zu gehen, saugen sie das Knowhow des Politikers, die Atmosphäre von Nagata-chô auf [Regierungsbezirk in Tôkyô d.A.]. Das ist die effektivste Weise zur Erzeugung von ,potentials’.“

Der Abgeordnete Usui, dessen Sohn zum Zeitpunkt der Befragung für das Regionalparlament von Chiba kandidierte, wies ebenfalls auf dieses Sprichwort hin: „Der Sohn eines Priesters kann diese Schriften lesen, weil er sie von klein auf gelesen hat, obwohl er keinen besonderen Unterricht dafür bekommen hat. Er behält die Sachen, obwohl er sie nicht speziell gelernt hat, im Kopf. [...] Die Kinder eines Politikers, die zweite Generation, hat keine Ausbildung hierfür bekommen, aber weil sie tagtäglich die Arbeit des Politikers sehen, haben sie auf jeden Fall das Gefühl dafür. Das heißt, für den Politiker selbst ist das natürlich sehr wünschenswert.“

Auf diese Weise, so erklärten die Interviewpartner, gebe es eine gewisse „natürliche“ Sicherheit bei der Wahlkreisarbeit oder im Wahlkampf. „Durch die Abstammung von einer Politikerfamilie, wenn der Vorgänger ein sehr guter Politiker war oder sich dem Wahlkreis sehr nahe gefühlt hat, bekommt man schon von Anfang an eine gewisse Sicherheit, wenn man diese Politik weiterführt. Auf dieser Ebene. In diesem Sinn, wenn der Vorgänger nichts Schlechtes getan hat und dem Wahlkreis sehr nahe stand, hat man vom ersten Schritt eine gewisse Sicherheit. Das ist der Grund für die Wahl von Erbabgeordneten“, sagte der Abgeordnete Kosaka. Der Erbfolger tritt also gewissermaßen in die Fußstapfen seines Vaters. Auch die Übernahme einer kôenkai ist dann deutlich einfacher.

Das gilt vor allem, wenn der Nachfolger bereits über eigene Erfahrungen in der Wahlkreisarbeit verfügt. Der hohe Anteil von Erbabgeordneten, die als Sekretär ihrer Eltern gearbeitet haben, ist kein Zufall. Der DPJ-Abgeordnete Watanabe: „Daher machen die meisten Abgeordneten ihre Söhne zu ihrem Sekretär, lassen ihn die eigene kôenkai kennen lernen, und lassen ihn so lernen. Zum Beispiel wurde Tanaka Makiko ${ }^{470}$ einst zur First Lady gemacht, ging nach Amerika und China und traf Nixon. Auf diese Weise ließen die Eltern ihre Kinder Erfahrungen machen. Zum Beispiel wurde Generalsekretär Fukuda auch mit 40 Jahren Sekretär seines Vaters. So bereitet man sie Schritt für Schritt vor, nicht wahr. Damit steigt auch langsam die Motivation, denke ich.“

\footnotetext{
${ }^{470}$ Die Tochter des ehemaligen Ministerpräsidenten Tanaka Kakuei, die später Außenministerin im Kabinett Koizumi wurde.
} 
Auch Park weist darauf hin, dass die Erfahrungen im Wahlkreis nach wie vor eine große Rolle bei der Kandidatenrekrutierung spielen. Da eine gut geführte kôenkai noch immer Vorraussetzung für eine erfolgreiche Kandidatur ist, haben es Kandidaten leichter, die bereits in der Wahlkreisarbeit erfahren sind. ${ }^{471}$

Den Erbfolgern stellt sich allerdings die Aufgabe, sich irgendwann von dem Vorbild zu lösen. Ein anderes Problem ist, dass dieses Hineinwachsen in die Politik natürlich auch eine Art Zwang darstellen kann, in die Politik gehen zu müssen. Im Zusammenhang mit dem eigenen Einstieg der Interviewpartner in die Politik wurde bereits darauf hingewiesen. Der Abgeordnete Kosaka bewertete das „Hineinwachsen in die Politik“ folgendermaßen: „Allerdings gibt es auch eine negative Seite, wenn man meint, dass es einen Zwang gibt, Erbabgeordneter werden zu müssen. Es gibt die Leute, die eigentlich in einem anderen Beruf arbeiten wollen, aber von der Politik sozusagen eingefangen werden. Wenn man es aber wirklich will, ist es eine gute Sache. Natürlich gibt es auch Erbabgeordnete, die es gerade deswegen [wegen des Zwangs d.A.] machen und eine hohe Motivation haben. “

Das Aufwachsen in einer Politikerfamilien stellt in diesem Fall also eine Motivation dar, diesen Beruf weiterzuführen. Der Abgeordnete Itô, der seinen Weg in die Politik als familiäre Pflicht ansah, betrachete dieses Pflichtgefühl als etwas Positives: „Wie bei dem Sohn eines Unternehmers, der sagt: ,Ich möchte das Unternehmen noch größer machen’, gibt es Kinder von Abgeordneten, die sagen: ,Ich möchte Japan verbessern.' Oder: ,Ich möchte zum Wohl der japanischen Bürger die Wirtschaft verbessern und zur Ehre eines unabhängigen Landes seine Kultur oder Wertvorstellungen aufbauen.'“

In welchem Umfang das Aufwachsen in einer Politikerfamilie eine Art Pflichtgefühl bei Erbfolgern weckt, lässt sich, wie bereits im ersten Teil angemerkt wurde, sicherlich nur am Einzelfall prüfen. Es ist aber bemerkenswert, dass fast alle Interviewpartner in diesem Punkt, unabhängig von der Parteizugehörigkeit oder dem Alter, übereinstimmten.

Nicht von der Hand zu weisen ist jedoch, dass die erworbenen Kenntnisse in der Wahlkreisarbeit, etwa im Rahmen einer Tätigkeit als Sekretär beim Vorgänger ein gute Voraussetzung darstellen, sowohl die Nachfolge einer Unterstützerorganisation anzutreten als auch die offizielle Anerkennung seitens der Partei zu erhalten. Deshalb stellt das „Hineinwachsen in die Politik“ auf der Angebotsseite auch unter den neuen politischen Rahmenbedingungen einen Vorteil für Erbpolitiker dar.

\footnotetext{
${ }^{471}$ Vgl: Park, Cheol-Hee: A.a.O., S. 53.
} 


\subsection{Zusammenfassung: Veränderungen auf der Angebots- und Nachfrageseite}

Die Veränderungen der Opportunitätsstruktur, vor allem des Wahlsystems und der Parteienfinanzierung haben sich auch auf die Rekrutierungsprozesse ausgewirkt. Auf der Nachfrageseite betreffen die zu beobachtenden Veränderungen vor allem die habatsu, die bei der Rekrutierung einen großen Teil ihres Einflusses zugunsten der Parteiführung eingebüßt haben. Wie von einigen Interviewpartnern angemerkt, hat es in erster Linie eine Machtverschiebung von den habatsu zur Parteizentrale gegeben. Die Parteizentralen scheinen sich bei strittigen Kandidaturen auch zunehmend gegenüber den persönlichen Unterstützerorganisationen durchsetzen zu können. Für die offizielle Aufstellung durch die Parteiführung sind persönliche Verbindungen und informelle Netzwerke jedoch nach wie vor sehr wichtig.

In diesem Sinne haben Erbabgeordnete zumindest in der LDP durch die langjährigen Beziehungen ihrer Vorgänger zur Parteiführung bessere Chancen als die Nicht-Erbabgeordneten. Außerdem stellen nach wie vor die kôenkai in vielen Wahlkreisen die Selektoren dar, die über eine Kandidatur bzw. Nachfolge der Unterstützerorganisation mitentscheiden. Ihre Untersützung spielt für eine erfolgreiche offizielle Nominierung eine weitaus größere Rolle als die zumeist recht schmale Parteibasis in den Wahlkreisen. Die erwartete Aufwertung der lokalen Parteiorganisationen ist in den konservativen Parteien bisher nur geringem Umfang erfolgt.

Zwar werden in vakanten Wahlkreisen verstärkt transparentere Auswahlmechanismen wie Vorwahlen durchgeführt, gerade die LDP scheint bisher jedoch nicht in der Lage gewesen zu sein, einheitliche Rekrutierungsmechanismen durchzusetzen. Somit haben sich hier alte Verhaltensmuster weitgehend erhalten haben. Auch bei der Besetzung der Parteilisten gibt es in der Regel informelle Absprachen, dennoch scheint hier tatsächlich eine Stärkung der Parteizentralen erfolgt zu sein. In der DPJ werden zwar Vorwahlen und öffentliche Auswahlverfahren bei der Kandidatenrekrutierung in größerem Umfang durchgeführt als bei den Liberaldemokraten, aber auch bei den Demokraten haben sich diese Mechanismen noch nicht vollständig durchgesetzt.

Auf der Angebotsseite verfügen Erbpolitiker mit ihren zumeist größeren Ressourcen ebenfalls über einige Vorteile gegenüber ihren Konkurrenten, sowohl bei der Kandidatenauswahl als auch in den Wahlkämpfen selbst. In den Einerwahlkreisen, vor allem in ländlichen Regionen, haben sich die Wahlkampfstile nicht grundlegend geändert. Die Einführung der Einerwahlkreise hat sogar eher dazu geführt, dass es für einen Herausforderer schwieriger geworden ist, sich gegen den Abgeordneten des Wahlkreises bzw. dessen Nachfolger durchzusetzen. Es ist jetzt nicht mehr möglich, mit einer relativ geringen Stimmenzahl über die hinteren Plätze ein Mandat zu bekommen. Wahlen in Japan sind nach wie vor personalisiert und bauen auf 
persönlichen Unterstützerorganisationen der Kandidaten auf. Zwar hat die Bedeutung der finanziellen Ressourcen leicht abgenommen, der Bekanntheitsgrad eines Kandidaten und die Stärke seiner Unterstützerorganisation sind immer noch wichtige Vorraussetzungen für eine erfolgreiche Kandidatur. Vor allem in der LDP sind deshalb die Chancen für Aspiranten, die nicht über entsprechende persönliche Unterstützergruppen, einen bekannten Namen und die finanziellen Mittel verfügen, gering, als offizieller Kandidat anzutreten.

Auch die Kandidatur auf einem Listenplatz bietet „Newcomern“ nur selten eine Alternative. Aussichtsreiche Listenplätze werden vor allem als Ausweichposition für altgediente Parlamentarier oder als Absicherung für Abgeordnete in den Einerwahlkreisen genutzt. Dabei werden Amtsinhaber bevorzugt behandelt, neue Kandidaten auf die hinteren Plätze gesetzt. Die Rekrutierungspfade in der japanischen Politik sind also vor allem in der LDP noch recht schmal und bieten Erbabgeordneten einige Vorteile. 


\section{Die Zukunft der Erbfolge in der japanischen Politik}

Offensichtlich bestehen immer noch Vorteile für Erbabgeordnete, sowohl auf der Nachfrageals auch auf der Angebotsseite. In diesem Kapitel soll der Frage nach der Zukunftsfähigkeit dieses Rekrutierungspfades nachgegangen werden. Interessant ist zum einen, ob die Abgeordneten eine Erbfolge in ihrem eigenen Wahlkreis in Betracht ziehen oder ob bereits eine Nachfolgeregelung getroffen wurde. Zum anderen stellt sich die Frage, ob die Interviewpartner den Rekrutierungspfad des Erbabgeordneten in Japan allgemein für zukunftsfähig halten. Im Schlussteil des Fragebogens sollten die Erbabgeordneten schließlich eine Einschätzung abgeben, ob sich die Erbfolge in der japanischen Politik halten wird. Haben die politischen Rahmenbedingungen, die Veränderungen auf der Nachfrage-, aber auch auf der Angebotsseite, dazu geführt, dass diese Form der Kandidatenrekrutierung abnehmen wird? Oder sind die Chancen für Erbfolge unter dem neuen politischen System nach wie vor groß?

Selbstverständlich handelt es sich bei diesen Einschätzungen um subjektive Sichtweisen der Befragten. An dieser Stelle zeigten sich aber die Vorteile einer qualitativen Befragung. Einige neue Aspekte, wie etwa die Probleme bei der Kandidatenrekrutierung oder der Kampf um so genannte potentials, konnten aufgegriffen und im Rahmen der Interviews weiter vertieft werden. Auch auf diese Punkte soll im folgenden Kapitel eingegangen werden, da sie teilweise eine Folge der Erbfolge darstellen und die Akteure auf der Nachfrageseite zu einem Nachdenken über die bisherigen Rekrutierungsmechanismen zwingen.

\subsection{Die Erbfolge im eigenen Wahlkreis}

Die Fragen zur Erbfolge im eigenen Wahlkreis wurden von den Interviewpartnern nur sehr zögerlich beantwortet. Alle befragten Abgeordneten behaupteten, sie hätten über eine Nachfolge noch nicht entschieden.

Man muss an dieser Stelle allerdings bedenken, dass viele der interviewten Erbabgeordneten noch nicht am Ende ihrer Karriere angelangt sind. Wie bereits im ersten Teil dargelegt wurde, gibt es in der japanischen Politik viele Abgeordnete, die bis weit über das Alter von 70 Jahren hinaus ein Mandat ausüben. Selbst der Abgeordnete Hori, der zum Zeitpunkt der Befragung 71 Jahre alt war, sagte, er habe noch keinen Nachfolger bestimmt. Betrachtet man die Biographien der einzelnen Abgeordneten und die Angaben zum eigenen Einstieg in die Politik, findet man viele Beispiele, bei denen eine Nachfolge sehr kurzfristig entschieden wurde, etwa beim Tod des Vaters, einem plötzlichen Rücktritt des Vorgängers usw. 
Lediglich drei Interviewpartner, die LDP-Abgeordneten Usui, Ôno und Kosaka, äußerten sich sehr eindeutig in dieser Frage. Bei den anderen Interviewpartnern wurde erst nach einigen Nachfragen eine Tendenz für oder gegen eine Nachfolge erkennbar.

Eine künftige Erbfolge ist nur im Fall von Usui Hideo offensichtlich. Sein Sohn, Usui Shôichi, trat 2003 bei den Wahlen zum Regionalparlament von Chiba an und gewann ein Mandat. Usui Hideo merkte allerdings an, dass eine Erbfolge nicht von Anfang an geplant war, begrüßte aber die Entscheidung seines Sohns: „Es war aber nicht so, dass ich sagte: ,Versuchen wir doch mal, ihn in die Politik zu bringen.' Ich denke, es zeugt von einem schlechten Politik-Stil, so etwas hinter dem Rücken des Betroffenen zu machen. Aber ich habe es natürlich begrüßt. Also in Zukunft ist mein Sohn da, nicht wahr. Wenn man es so überlegt, ist es eigentlich ganz gut so, denke ich. “ Ob sein Sohn später einmal für das Unterhaus kandidiert, ließ er jedoch offen.

Die Kandidatur von Usui Shôichi ist dennoch ein gutes Fallbeispiel für eine mögliche Erbfolge. Der Regionalpolitiker hatte sich 2002 kurzfristig entschieden, als Kandidat für das Regionalparlament von Chiba anzutreten. Da es bisher keinen Kandidaten der LDP in diesem Wahlkreis gab, war es für ihn relativ einfach, die Anerkennung als offizieller LDP-Kandidat zu erhalten. Erleichternd kam sicherlich hinzu, dass sein Vater gleichzeitig Parteivorsitzender von Chiba ist.

Zunächst stand der junge Abgeordnete einer politischen Laufbahn jedoch überwiegend kritisch gegenüber: „Seit ich mich erinnern kann, ist mein Vater in der Politik, nicht wahr. Als ich etwa vier oder fünf Jahre alt war, wurde mein Vater Parlamentsabgeordneter. Als ich klein war, sah ich, was für ein anstrengendes Leben mein Vater führt, und ich wollte niemals Politiker werden. Ich wollte auf keinen Fall Politiker werden. “ Usui Shôichi arbeitete zunächst als Angestellter bei Tôkyô Disney Land.

Usui Hideo selbst gab sich allerdings überrascht über die Entscheidung des Sohnes: „Ich habe ihn nicht direkt danach gefragt. Ich habe ihn nicht einmal danach gefragt, ob er sich irgendwann einmal aufstellen lassen will. Daher war ich etwas überrascht, als er sagte, er wolle sich diesmal aufstellen lassen. Aber wie ich es sehe, schätzt er, dass er so die Gesellschaft mitgestalten kann. Er denkt, er könnte es auf ähnliche Weise machen, wie er es bei mir gesehen hat.“

Hier zeigt sich wieder das Motiv des „Hineinwachsens“ in die Politik. Usui Hideo fügte hinzu, sein Sohn habe auch eine politische Laufbahn eingeschlagen, weil er bei ihm von klein auf „hinter die Kulissen“ habe blicken können. 
Von großer Bedeutung während der Wahl war die logistische Unterstützung. Der Wahlkreis von Usui Shôichi ist Teil des Unterhaus-Wahlkreises seines Vaters. Die Wahlkampforganisation des Nachwuchspolitikers war folglich überwiegend „geliehen“. Usui Shôichi sprach in diesem Zusammenhang von einer Chance, die er ergriffen habe. Sein „Umfeld“ habe es ihm erlaubt, zu kandidieren. Bemerkenswert ist in diesem Zusammenhang auch, dass er in seinem Wahlkreis, auf regionaler Ebene existieren noch die Mehrerwahlkreise, den ersten Platz vor dem bisherigen Amtsinhaber belegte.

Am Beispiel dieses Regionalpolitikers zeigen sich also deutlich die Vorteile einer Erbfolge, die wohl vor allem auf lokaler und regionaler Ebene eine große Rolle spieln. Zum einen schafft das Aufwachsen in einer Politikerfamilie eine gewisse Nähe zur Politik und vermittelt auch wichtige Kenntnisse in der Wahlkreisarbeit. Zum anderen bestehen sowohl auf der Nachfrageseite als auch auf der Angebotsseite gewisse Vorteile. Eine offizielle Kandidatur erscheint durch die Kontakte zur Partei leichter und auch die logistische Unterstützung ist gesichert.

Zwei Interviewpartner schlossen eine Erbfolge in ihrem Wahlkreis aus. Ôno Yoshinori begründete es damit, dass es keinen geeigneten Nachfolger in seiner Familie gebe. Sein Sohn habe kein Interesse an der Politik: „Wenn sich der Sohn aber nicht so für die Politik interessiert, sollte er einen anderen Weg gehen. Zum Beispiel sollte die Familie Ôno einmal Pause machen... Das Zeitalter, in dem der Sohn Politiker wurde, weil der Vater es war, geht langsam vorbei, nicht wahr. “

Daher habe er seine Nachfolge noch nicht geregelt. Allerdings äußerte Ôno Kritik an der Nachfolgeregelung innerhalb der kôenkai: „Früher hat immer die kôenkai entschieden. Wenn die Familie den Sohn schickte, wurde er meist von der kôenkai unterstützt. Aber das muss aufhören. Meiner Meinung nach. “

Auch der Abgeordnete Kosaka lehnte eine Erbfolge ab. Er werde auf keinen Fall einen Verwandten antreten lassen. Zu den Gründen äußerte er sich nicht.

Die anderen Abgeordneten gaben an, sie hätten noch keine Nachfolgeregelung getroffen. Aber auch sie hielten mehrheitlich eine Erbfolge für schwierig. Dafür wurden sehr unterschiedliche Gründe genannt. Bei einem Teil der Abgeordneten scheint es im Verwandtenkreis keinen geeigneten Nachfolger zu geben. Der Abgeordnete Hori: „Ein Nachfolger ist meine größte Sorge, nicht wahr. Wenn ich einen Sohn hätte, würde ich darüber nachdenken. Aber ich habe ja keinen Sohn. Ich habe Töchter. Zwei Mädchen. Sie sind beide verheiratet und tragen ja nicht 
mehr den Namen Hori. Und die Männer haben beide ihren Beruf. Also habe ich überhaupt keine Absichten, sie zu Politikern zu machen. Überhaupt nicht. Es geht ja gar nicht. “

Andere Interviewpartner schätzten die Chancen für eine Erbfolge in ihrem Wahlkreis als sehr gering ein, selbst wenn es potenzielle Nachfolger gibt. Der Abgeordnete Hamada sagte beispielsweise, er wolle seinen Kindern unter den derzeitigen Bedingungen nicht empfehlen, eine politische Karriere einzuschlagen. Auch Watanabe Shû sagte, eine Erbfolge habe nicht zuletzt durch die Veränderungen im Wahlkreis nur eine geringe Aussicht auf Erfolg. Vor allem wegen der sich verändernden Bevölkerungsstrukturen in seinem Wahlkreis (s.o.) würden sich immer weniger Wähler mit dem Vorgänger verbunden fühlen. Eine Erbfolge erschien ihm deshalb kaum möglich.

Es stellt sich die Frage, wie im Fall der befragten Erbabgeordneten über eine Nachfolge entschieden wird. Interessanterweise scheint es, dass, selbst wenn eine Erbfolge nicht beabsichtigt oder als wenig wahrscheinlich betrachtet wird, die Interviewpartner bei der eigenen Nachfolge mitbestimmen möchten. Der Abgeordnete Ishiba dazu: „Es gibt ja überhaupt keine geeigneten Leute. Ich habe zwar schon sechsmal die Wahl gewonnen, bin aber erst 48 Jahre alt. Normalerweise macht man etwa 20 Jahre lang diese Arbeit. Also ich habe noch keinen Nachfolger bestimmt, aber ich möchte möglichst früh jemanden als meinem Nachfolger aufbauen. Wenn möglich, soll er mit mir zusammen einen Wahlkampf führen, so lange ich noch Abgeordneter bin. Ich möchte jemanden aufstellen, der von meinen Vorstellungen nicht allzu sehr abweicht.“

Das Aufbauen eines Nachfolgers erscheint als sehr wichtig. Angesichts der politischen Rahmenbedingungen hat es ein Nachfolger, der nicht nur über Erfahrungen im Wahlkreis verfügt, sondern über auch einen bekannten Namen, etwas leichter als andere Kandidaten.

Die Abgeordneten sprachen allerdings davon, dass, selbst wenn eine Nachfolge in der kôenkai beschlossen ist, eventuell Schwierigkeiten auftreten, den Nachfolger gegenüber der Parteizentrale durchzusetzen. Wie bereits gezeigt wurde, haben die Parteizentralen nicht nur gegenüber den habatsu sondern, wenn auch in geringerem Maße, gegenüber den persönlichen Unterstützervereinigungen an Einfluss bei der Kandidatenrekrutierung gewonnen.

Der Abgeordnete Funada stellte den Auswahlprozess folgendermaßen dar: „Ja, wenn man das unter dem Aspekt der Erbfolge sieht... also ich habe ja einen Sohn. Aber er hat kein Interesse, ihn [den Wahlkreis - d.A.] zu übernehmen. Wenn er sagen würde, dass er das unbedingt machen möchte, würde ich ihn der kôenkai vorstellen. Danach würde ich das Urteil der kôenkai abwarten, aber von meiner Seite würde kein Druck ausgeübt werden. Ich werde nichts er- 
zwingen. Das heißt, ich habe auch nicht die Absicht, von der Parteizentrale zu fordern, dass mein Sohn auf jeden Fall aufgestellt wird. “

Die Abgeordneten suchen daher eine Lösung, mit der alle, sowohl die kôenkai als auch die Parteizentrale einverstanden sind. Der Abgeordnete Hori wies auf einige negative Beispiele hin, bei denen eine Nachfolge nur von einem Teil der Beteiligten mitgetragen wurde. So kam es beispielsweise zu Machtkämpfen zwischen Partei und kôenkai oder auch zwischen Teilen der Unterstützerorganisation. Deshalb sei ein Konsens wichtig: „Man kann also nicht wie ein König entscheiden, weil man ja eine Verantwortung hat. Deshalb muss man den Nachfolger so auswählen, dass sich niemand beschwert. In meinem Fall ist es ähnlich. Wenn ich einen Kandidaten ausgewählt habe, muss ich sichergehen können, dass alle, also auch die anderen Interessenten [, die unterlegen sind - d.A.], zusammen für die LDP kämpfen, und dass es über seine Wahl keine Klagen gibt.“

Eine Rolle dürfte sicherlich auch spielen, dass in einigen Wahlkreisen inzwischen Vorwahlen durchgeführt werden. Der Abgeordnete Watanabe glaubte, dass seine Nachfolge wohl in einer Vorwahl entschieden werde, obwohl er eine Erbfolge nicht gänzlich ausschloss. Allerdings kann auch im Fall einer Vorwahl die Zusammensetzung des Auswahlgremiums eine Erbfolge begünstigen, wie bereits dargelegt wurde.

Die Fragen zur Erbfolge im eigenen Wahlkreis wurden also von vielen Interviewpartner ausweichend beantwortet. Lediglich in drei Fällen (Ôno, Usui, Kosaka) gab es eindeutige Stellungnahmen. Aber selbst Usui Hideo, in dessen Fall man eine geplante Erbfolge durch den Sohn erkennen konnte, mochte sich nicht festlegen, ob sein Sohn einmal für das Unterhaus kandidieren wird.

Interessanterweise schlossen die DPJ-Abgeordneten, mit Ausnahme von Terata Manabu, der ja zur Zeit der Befragung erst 28 Jahre alt war, eine Erbfolge nicht kategorisch aus. Trotz der Anstrengungen, transparentere Auswahlmechanismen in der DPJ zu etablieren, scheint eine Erbfolge auch in der Demokratischen Partei weiterhin möglich.

Da auf die anderen Fragen, soweit es nachprüfbar war, recht offen geantwortet wurde, stellt sich also die Frage, ob eine Nachfolge tatsächlich sehr kurzfristig entschieden wird oder ob die Interviewpartner sich zu dieser sehr persönlichen Frage nicht äußern wollten. Allerdings gibt es, etwa im Fall des japanischen Ministerpräsidenten Koizumi, durchaus viele Beispiele dafür, dass ein Familienmitglied, etwa beim Tod des Abgeordneten, kurzfristig eine andere berufliche Tätigkeit beendet und eine Erbfolge antritt. 


\subsection{Das Ende eines Rekrutierungspfades?}

Zur Zukunft der Erbfolge gab es auf Seiten der Interviewpartner höchst unterschiedliche Ansichten. Angesichts der oben dargestellten Veränderungen auf der Nachfrage- und Angebotsseite, kann man davon ausgehen, dass die Vorteile einer Erbfolge zumindest abnehmen, auch wenn dieser Rekrutierungspfad immer noch Relevanz besitzt.

Tatsächlich äußerten acht Abgeordnete, darunter die DPJ-Abgeordneten, die Meinung, dass die Chancen für eine Erbfolge gesunken sind. Das wurde vor allem mit dem Wahlsystem in Verbindung gebracht. Der Abgeordnete Funada dazu: „Ich denke, auf der Grundlage des neuen politischen Systems wird die Zahl der Abgeordneten, die eine Nachfolge antreten, langsam abnehmen. Wie ich ja bereits sagte, nimmt der Einfluss der kôenkai ab und der Einfluss der Parteizentrale nimmt zu. “

Folge dieser Zentralisierungstendenzen sei, dass die Parteien einen immer größeren Einfluss auf die Kandidatenrekrutierung nehmen. Ein Nachfolger könne deshalb nicht mehr einfach „durchgewunken“ werden. Interessanterweise war auch der Abgeordnete Usui, bei dem eine Erbfolge durch den Sohn absehbar ist, der Meinung, dass die Chancen dieses Rekrutierungspfades gesunken sind.

Der Abgeordnete Kosaka vertrat ähnliche Ansichten: „Wie ich bereits sagte, wird diese Zahl abnehmen. Mit dem Wandel [...] zum parteizentrierten System hat sich ja auch das Bewusstsein der Wähler verändert. Das politische Programm ist wichtiger geworden. Man schaut nicht nur auf die Person, sondern auch auf das politische Programm. Die Einführung eines ,Manifests' durch Koizumi ist ein gutes Beispiel. Auf der Grundlage dieses Wandels haben die Vorteile der Erbabgeordneten gegenüber den jeweiligen anderen Kandidaten abgenommen. “

Die Zahl der Erbabgeordneten werde dementsprechend sinken. Das heißt aber nicht, dass dieser Rekrutierungspfad gänzlich verschwinden wird. Der Abgeordnete Harada: „Bei den Wahlen vor Ort bestehen bestimmt noch einige Anreize. Aber es ist nicht mehr unbedingt der Fall wie früher. Sicherlich werden einige, ,one of them', gewählt werden. Es ist schließlich immer noch einfacher eine kôenkai zu übernehmen. Aber weil die Wähler viel mehr Auswahlmöglichkeiten haben, ist das nicht mehr zwangsläufig der Fall.“

Drei der Interviewpartner sagten, die Chancen für eine Erbfolge seien nach wie vor recht hoch. Die Zahl der Erbabgeordnete werde daher zumindest mittelfristig stabil bleiben. Sie nahmen an, dass es für Politiker der zweiten oder dritten Generation immer noch einfacher sei, Wahlkämpfe zu führen. Die Zahl der Erbabgeordneten werde daher auf hohem Niveau gleich bleiben. 
Die beiden Interviewpartner Itô Shintarô und Usui Shôichi sprachen sogar von erhöhten Chancen für eine Erbfolge. Itô Shintarô: „Wenn man das 1955er-System mit heute vergleicht, dann ist es unter dem Aspekt von Mehrerwahlkreis und Einerwahlkreis für Erbabgeordnete in der LDP heute leichter."

Dementsprechend werde sich künftig auch die Zahl der Erbabgeordneten erhöhen. Auch Usui Shôichi sagte, dass ein für Erbabgeordnete vorteilhaftes Wahlsystem eingeführt wurde. Die beiden Abgeordneten wiesen in diesem Zusammenhang auf die Stellung des Abgeordneten im Einerwahlkreis hin. Für Herausforderer ist es anscheinend sehr viel schwieriger geworden, gegen den Amtsinhaber zu gewinnen. Ein Nachfolger, der etwa im Fall einer Erbfolge von einem Amtsinhaber den Wahlkreis übernimmt, habe deshalb größere Chancen, ein Mandat zu gewinnen.

Ein Wahlsystem mit Einerwahlkreisen verringert also nicht zwangsläufig die Chancen einer Erbfolge, sondern verstärkt sie unter Umständen sogar. Der Eintritt in die Politik ist nach Otake durch die außergewöhnliche Stärke des Amtsinhabers für alle neuen Kandidaten schwierig. Eine Ausnahme bilden lediglich die Erbpolitiker. ${ }^{472}$ Vor diesem Hintergrund sind die Aussagen der beiden Interviewpartner durchaus plausibel.

An dieser Stelle wurde aber auch darauf hingewiesen, dass gewisse Unterschiede zwischen DPJ und LDP bestehen. Darauf wird im folgenden Kapitel bei der Betrachtung der Probleme bei der Kandidatenrekrutierung aber noch näher eingegangen. Bezeichnend ist jedoch, dass alle DPJ-Abgeordneten von einer Abnahme der Erbfolge ausgingen.

Allerdings muss man an dieser Stelle die Aufstellung als offizieller Kandidat von den allgemeinen Wahlchancen trennen. In der Wahl selbst haben nach Ansicht der meisten Interviewpartner die Erbabgeordneten tatsächlich gewisse Vorteile. Bei der Frage nach den Zukunftschancen dieses Rekrutierungspfades gab es je nach Parteizugehörigkeit Unterschiede in den Antworten, die sich aber vor allem auf die eigentliche Auswahl der offiziellen Parteikandidaten bezogen.

Die DPJ-Kandidaten wiesen daraufhin, dass jetzt Kandidaten, die über keine familiären Verbindungen in die Politik verfügen, bessere Chancen als unter dem alten Wahlsystem hätten, als offizielle Kandidaten aufgestellt zu werden: „Herr Hatoyama [ehemaliger Parteivorsitzender d.A.] war Erbabgeordneter, aber Herr Kan [Parteivorsitzender zur Zeit des Interviews - d.A.] und Herr Okada und die jungen Abgeordneten, die jetzt eingezogen sind, diese Leute sind überhaupt keine Erbabgeordneten. Wenn diese Leute neue Mechanismen in der Politik schaffen,

\footnotetext{
${ }^{472}$ Vgl.: Otake, Hideo: A.a.O., S. 281.
} 
denke ich, werden sie auch ein ,Nein' zu Erbabgeordneten etablieren. Daher glaube ich, dass in der DPJ schneller als in der LDP ein System geschaffen wird, das ,Nein’ zu Erbabgeordneten sagt, “ so der Abgeordnete Watanabe. Hier sei man schon wesentlich weiter als in der LDP.

Doch selbst wenn der Zugang zu einer Kandidatur für andere Kandidaten erleichtert wurde, haben die Erbabgeordneten immer noch die Chance, parteilos anzutreten: „Allerdings gibt es auch Erbabgeordnete, die als Unabhängige gewinnen, selbst wenn sie keine offiziellen Kandidaten sind“, räumte Watanabe ein.

Im Idealfall verfügen die Erbabgeordneten über eine starke kôenkai und genügend finanzielle Ressourcen, um den offiziellen Kandidaten zu schlagen. Nicht zuletzt aus diesem Grund waren auch die Interviewpartner, die von einer Abnahme der Erbabgeordneten sprachen, der Ansicht, dass das Phänomen der Erbabgeordneten auch in Zukunft in ländlichen Regionen weiter existieren wird. Watanabe: „Es gibt Gegenden, in denen die Kinder die dritte Generation bilden. Zum Beispiel waren auch die Väter von Koizumi und Ôzawa Ichirô Politiker. Von den derzeit prominenten Parteiführern sind nur Frau Doi von der SDPJ und Herr Kan von der DSP keine Erbabgeordneten. Die Väter der anderen, Hashimoto oder Hatoyama, waren ebenfalls Politiker. Leider verfestigt sich das ohne einen Wandel im System des Politikfinanzierung und der kôenkai in den Wahlkreisen. Wahrscheinlich gibt es die Tendenz, dass die japanische Gesellschaft Außenseiter ablehnt. Wenn man in die Gemeinden geht, verstärkt sich diese Tendenz noch mehr. Kurz gesagt, es ist schwer für neue Leute anzutreten, und so können sie leider nicht kandidieren."

Allerdings weist Park darauf hin, dass eine Kandidatur als unabhängiger Kandidat unter dem neuen Wahlsystem ein Risiko darstellt, da die die nachträgliche Aufnahme in die Fraktion einer Partei wesentlich schwieriger geworden ist. ${ }^{473}$

Die Abgeordneten nannten vor allem Gebiete mit „dörflicher Struktur“ wie Kyûshû oder Hokkaidô, in denen dieser Rekrutierungspfad wohl erhalten bleibt, wohingegen Erbfolgen in städtischen Wahlkreisen schwieriger werden. Nach Klein sind es ja vor allem die ländlichen und semi-urbanen Wahlkreise, in denen die kôenkai und intensive Kontakte zur lokalen Wirtschaft für einen Wahlerfolg maßgeblich sind. ${ }^{474}$

Umgekehrt können Erbpolitiker in städtischen Wahlkreisen ihre Vorteile nicht im vollem Umfang ausspielen. Darauf wies auch der Abgeordnete Usui Hideo bei der Analyse des Wahlsieges seines Sohns hin. Dessen Wahlkreis Mihama-ku besteht vor allem aus Neubaugebieten, in denen junge, neu zugezogene Familien wohnen. Viel wichtiger als der bekannte Name der Familie Usui sei daher das jugendliche Auftreten des Sohnes (28 Jahre) gewesen. Allerdings

\footnotetext{
${ }^{473}$ Vgl.: Park, Cheol-Hee: A.a.O., S. 52.

${ }^{474}$ Vgl.: Klein, Axel: A.a.O., S. $279 f$.
} 
besteht auch kein Zweifel daran, dass ein Wahlkampf ohne die Unterstützung der kôenkai des Vaters kaum möglich gewesen wäre.

Interessant ist auch ein Blick auf die Zahl der Erbfolgen seit Einführung des neuen Wahlsystems. Im Jahr 2003 wurden 102 neue Abgeordnete in das Unterhaus gewählt, darunter befanden sich 13 Erbabgeordnete, die sich allerdings nur auf zwei Parteien verteilten, die LDP und die DPJ.

\section{Abbildung 20: Neu gewählte Erbabgeordnete 2003 (43. Legislaturperiode)}

\begin{tabular}{l||c|c|c|c|c|c|c||c}
\multicolumn{1}{c|}{ Partei } & LDP & DPJ & SDPJ & Kômeitô & KPJ & Sonst. & Unabh. & Gesamt \\
(Abg. insg.) & $(249)$ & $(176)$ & $(6)$ & $(34)$ & $(9)$ & $(1)$ & $(5)$ & (480) \\
\hline Abgeordnete & 35 & 58 & 2 & 3 & 1 & - & 3 & 102 \\
\hline Erbabgeordnete & 9 & 4 & - & - & - & - & - & 13 \\
\hline $\begin{array}{l}\text { Anteil Erbab- } \\
\text { geordnete }\end{array}$ & $25,7 \%$ & $6,9 \%$ & - & - & - & - & - & $\mathbf{1 2 , 7 \%}$
\end{tabular}

Nach: Saitô, Keiko: Seikanyôran, Vol. 22, No. 50, Seisakujihô Ai Pi, Tôkyô 2003 (Saitô, Keiko: Führer durch Politik und Bürokratie.), eigene Berechnungen.

In der LDP ist der Anteil der Erbabgeordneten an den neu gewählten Abgeordneten deutlich höher als in der DPJ, was den Schluss zulässt, dass diese Form der politischen Rekrutierung in der LDP immer noch gute Chancen besitzt. Betrachtet man die Zahl der Erbfolgen in den Jahren nach den politischen Reformen, ergibt sich folgendes Bild:

\section{Abbildung 21: Neu gewählte Erbabgeordnete 1993-2003}

\begin{tabular}{l||c|c|c|c} 
Legislaturperiode & 40. (1993) & 41. (1996) & 42. (2000) & 43. (2003) \\
\hline \hline Neu gewählt & 15 & 12 & 24 & 13
\end{tabular}

Nach: Saitô, Keiko: Seikanyôran, Vol. 20, No. 47, Seisakujihô Ai Pi, Tôkyô 2002 (Saitô, Keiko: Führer durch Politik und Bürokratie.), Saitô, Keiko: Seikanyôran, Vol. 22, No. 50/2004, Seisakujihô Ai Pi, Tôkyô 2004 (Saitô, Keiko: Führer durch Politik und Bürokratie.), eigene Berechnungen. 
Im Vergleich zum alten Wahlsystem hat sich die Zahl der Erbfolgen auf einem weitaus niedrigeren Niveau eingependelt. Allerdings ist auch hier wieder eine Art ten-year-cycle zu erkennen, dessen Höhepunkt das Jahr 2000 mit 24 neu gewählten Erbabgeordneten darstellt.

\section{Abbildung 22: Zahl der Erbfolgen (1958-2003)}

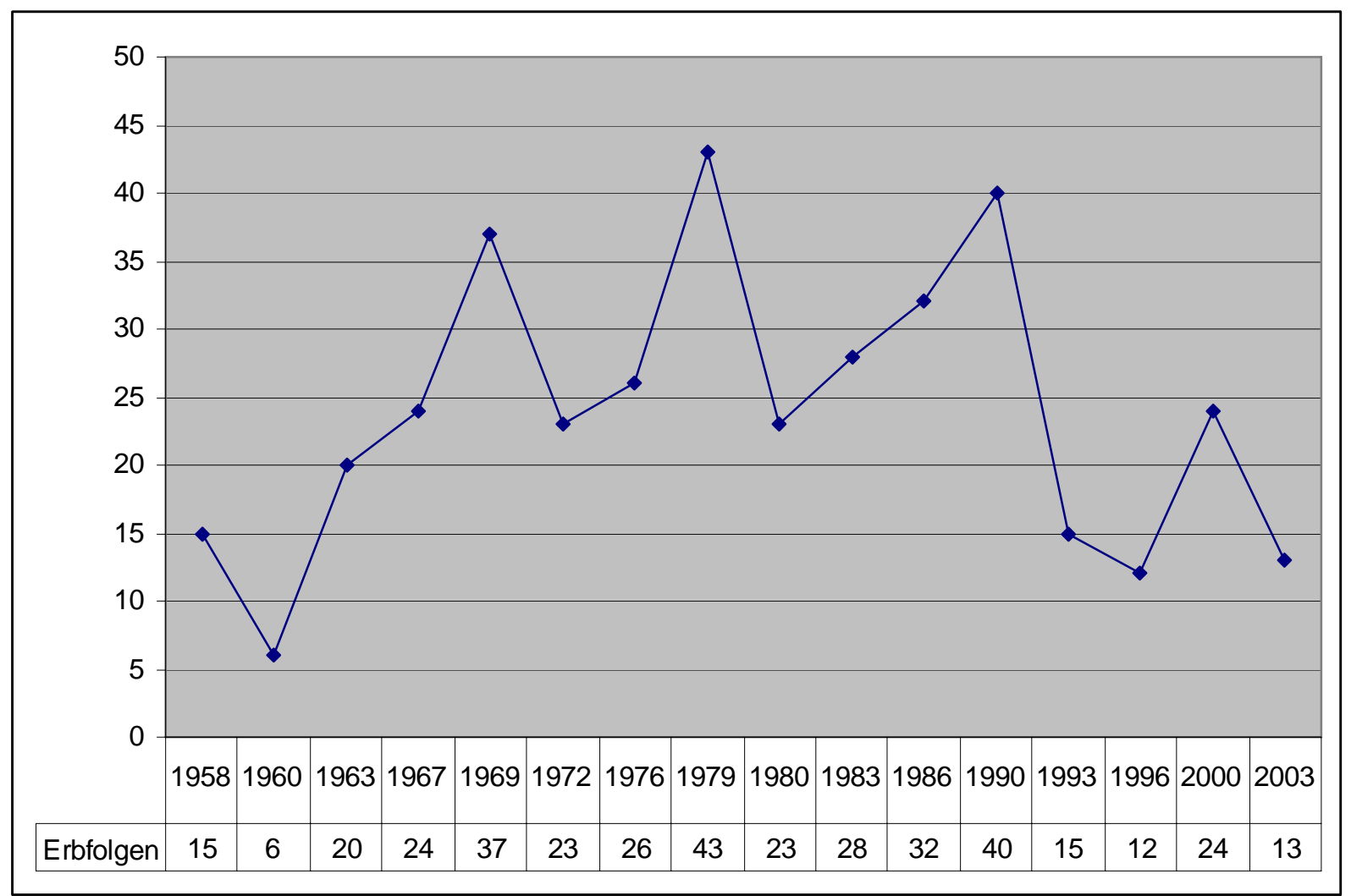

Nach: Ishibashi, Michihiro; Steven R. Reed: Second-Generation Diet Members and Democracy in Japan, in: Asian Survey, Vol. 32, No. 4/1992, S. 372; eigene Berechnungen.

Die Erbfolge verliert nach Ansicht vieler Interviewpartner also langsam an Relevanz. Einige Abgeordnete stellten diese Veränderung als ein gesamtgesellschaftliches Phänomen dar: „In Japan gibt nämlich die Mentalität, dass man eine sichere Nachfolge in Form einer Erbfolge wünscht, egal ob in einer Firma, bei einer Wahl oder in irgendeiner Organisation auf dem Land. So eine Mentalität gibt es, glaube ich. In letzter Zeit hat sich das bei den Unternehmen geändert und Erbfolgen sind seltener geworden. Es gibt einige Fälle, in denen Unternehmer, die eine Erbfolge angetreten haben, versagt haben. Gerade die großen Unternehmen haben sich jetzt gewandelt. Gleichzeitig ist das auch bei den Erbabgeordneten passiert. Seit der Einführung der Einerwahlkreise werden Kandidaten auf verschiedene Weise bestimmt, z.B. werden sie von der Partei empfohlen. So sind also die Möglichkeiten der Erbfolge eingeschränkt 
worden. Das hat sich sehr stark verändert“, so der Abgeordnete Kosaka. Eine Erbfolge auf den Parteilisten hielten die Interviewpartner darüber hinaus für unwahrscheinlich.

Mehrere Abgeordnete sprachen davon, dass es unter den Wählern verstärkt ein Bewusstsein gibt, dass Politik nicht vererbbar sein sollte. In diesem Sinn hängt also die die erfolgreiche Kandidatur eine Erbpolitikers in Zukunft von seiner tatsächlichen Stärke im Wahlkampf ab. Trotz dieser Tendenzen, darin waren sich die Abgeordneten einig, werde sich dieser Rekrutierungspfad aber zumindest in den ländlichen Gebieten halten können: „Die Zahl wird abnehmen, aber es wird nicht so sein, dass die Erbabgeordneten als Phänomen verschwinden. Wahlen sind immer noch sehr personenzentriert, daher wird das Phänomen der Erbabgeordneten auch weiterhin Bestand haben, “ so der Abgeordnete Ôno. 


\subsection{Die Bewertung der politischen Reformen}

\subsubsection{Unvollendet und uneffektiv - Kritik an den politischen Reformen}

Von den LDP-Abgeordneten wurden die politischen Reformen und die bisherigen Auswirkungen überwiegend als nicht erfolgreich und unvollendet betrachtet. Itô Shintarô: „Obwohl ich nicht verstehe, was die positiven Seiten sein sollen, denke ich, dass die Reformen noch unvollendet sind. “

Im einzelnen wurde Kritik vor allem an den Veränderungen in den Wahlkreisen geübt. Wegen der bereits erwähnten außergewöhnlich starken Stellung des Abgeordneten im Einerwahlkreis, hätten die Reformen negative Auswirkungen gehabt, da Japan nach wie vor sehr zentralistisch organisiert sei. Unter diesem Gesichtspunkt ist auch die Kritik Hamadas an den politischen Reformen zu sehen. Er kritisierte, dass es neben der Einführung der Einerwahlkreise keine Dezentralisierung, etwa bei der Vergabe der Budgets durch die Zentralregierung, gegeben habe: „Wenn man Dezentralisierung und Einerwahlkreise nicht gemeinsam einführt, heißt das zwangsläufig, dass die Macht sich bei einer Person konzentriert." In diesem Zusammenhang wurde auch die bereits erwähnte Vernachlässigung von Minderheiteninteressen kritisiert.

Interessanterweise wurde von den meisten LDP-Abgeordneten das System der Parteilisten kritisiert. Hier wurde häufig das bereits genannte Argumente geäußert, ein Abgeordneter, der über die Parteiliste ein Mandat erhalten hat, sei mit dem Wahlkreis nicht ausreichend verbunden. Aber auch seitens der Öffentlichkeit wurde seit ihrer Einführung die Art und Weise, wie die Parteilisten benutzt wurden, um Kandidaten abzusichern, kritisiert. ${ }^{475}$

Usui Hideo fasste zusammen, dass die Ziele des Reformpaketes nicht erreicht worden seien. Weder habe es eine Verjüngung der Parteien gegeben, noch eine Beseitigung der habatsu oder mehr parteipolitische Auseinandersetzung in den Wahlkämpfen. Auch der prophezeite Wandel zu einem Zwei-Parteien-System sei nicht eingetreten. Der Abgeordnete Ishiba, der ja während des Reformprozesses eine größere Rolle spielte, sprach davon, dass sich die hohen Erwartungen, die man in die Reformen gesetzt hatte, nicht erfüllt hätten.

Bei den Antworten zur Frage zu den Veränderungen in der Kandidatenrekrutierung lässt sich durchaus ein Bezug zu der Parteizugehörigkeit der Interviewpartner herstellen. Die Folgen der politischen Reformen für die Kandidatenrekrutierung wurden von den LDP-Abgeordneten überwiegend als negativ betrachtet. Das Ziel, den Zugang zur Politik zu erleichtern und eine (LDP-) Kandidatur einem größeren Personenkreis zu ermöglichen, scheint also nicht erreicht

\footnotetext{
${ }^{475}$ Vgl.: Jain, Purnendra C.; Todhunter, Maureen: The 1996 General Election: Status Quo or Step forward? In: Jain, Purnendra C.; Inoguchi, Takashi: Japanese Politics Today. Beyond Karaoke Democracy? St. Martin’s Press, New York 1997, S. 227.
} 
worden zu sein. Die Strukturen des 1955er-Systems haben sich nach Aussage zweier Abgeordneter (Hamada, Itô) sogar in einigen Bereichen verfestigt. Vor allem das Fehlen einer einheitlichen Regelung zur Kandidatenbestimmung hat demnach zu keiner wesentlichen Veränderung nach den politischen Reformen geführt. Die Folge ist, dass die Rekrutierung weiterhin unter den bereits im ersten Teil genannten Personengruppen stattfindet. Junge Talente können auf diese Weise in der LDP kaum gehalten werden.

Die genannten Zentralisierungstendenzen wurden von einigen Abgeordneten im Hinblick auf die fehlenden einheitlichen Regelungen zur Kandidatenbestimmung negativ bewertet. „In diesem Fall greifen die Parteien entschlossen nach der Führung, aber wenn sie keine Vorschriften dazu (zur Kandidatenrekrutierung - d.A.) machen, wird es auch nicht besser werden “, so der Abgeordnete Hamada. An diesem Punkt muss man aber eher von einem Versagen der Parteien als von einem Scheitern der Reformen sprechen.

Lediglich ein kleiner Teil der befragten Erbabgeordneten betrachtete die Auswirkungen der politischen Reformen auf die Kandidatenauswahl hingegen als positiv. Funada sprach davon, dass die Kandidatenrekrutierung sich verbessert habe. Durch die Einführung der Einerwahlkreise würden nun verstärkt Kandidaten ausgewählt, die für die Parteien vorteilhaft wären: „Die Wahrscheinlichkeit wird immer größer, dass von der Partei Kandidaten auswählt werden, die geeignet sind, bei der Wahl ihren Gegner zu schlagen. Natürlich gibt es auch welche, die ihre eigenen Parteifreunde attackieren, also zum Beispiel ,Koizumi hat da ein paar sehr komische Sachen gesagt' oder so und eine Gegenposition einnehmen. Dieses Problem gibt es. Aber es werden wohl geeignete Kandidaten ausgewählt, die für die Partei Vorteile mitbringen, und die auch gegenüber ihren Gegnern Vorteile haben. Ich denke, dass sich bald die Effizienz dieses Systems zeigen wird.“

Der Abgeordnete Ôno konnte zwar ebenfalls noch keine grundlegenden Veränderungen bei der Kandidatenrekrutierung feststellen, sagte aber, dass es noch zu früh sei, die politischen Reformen abschließend zu beurteilen: „, Wie weit sich das alles auf die Politikerfamilien auswirkt, das heißt auf die Erbabgeordneten, das ist noch in der Testphase. Dass es also soweit kommt, dass in den Wahlkreisen die Wähler sagen: ,Ah, das ist der Sohn, das geht aber nicht.' Dass man sagt: ,Das System der Erbfolge ist nicht gut.' Das wäre ein Fortschritt. Aber das ist nicht die Norm. Das befindet sich noch in der Testphase. “

Die Abgeordneten der DPJ hingegen stellten ihre Partei als Reformpartei dar, in der die Kandidatenauswahl wesentlich transparenter sei. Hier hätten auch Quereinsteiger und „normale Leute“ eine Chance, aufgestellt zu werden. Bei dieser Art der Selbstdarstellung muss man frei- 
lich vorsichtig sein. Tatsache ist, dass es auch in der DPJ höchst unterschiedliche Regelungen zur Kandidatenbestimmung gibt und die Folgen daher unterschiedlich eingeschätzt werden.

Die beiden DPJ-Abgeordneten äußerten die Meinung, dass die Reformen für die Kandidatenrekrutierung positive Folgen gehabt hätten. Das gelte besonders für ihre Partei, die einem größeren Personenkreis offen stünde als die LDP. Die Chancen für Kandidaten, die keine kôenkai mitbringen, seien höher. In diesem Sinne hätten die politischen Reformen Erfolg gehabt: „Wie gesagt, in den Mehrerwahlkreisen war die Erbfolge ein Vorteil. Heute ist eine Zeit angebrochen, in der man als guter Kandidat auch gewinnen kann, wenn man kein Zweitgenerationspolitiker oder Erbpolitiker ist. Jetzt drängen viele verschiedene Leute in die Politik. Auch ohne jiban, kanban, kaban kann man einen Sieg erringen. Ganz verschiedene Leute, wenn sie fähig sind, kommen in die Politik. Das ist eine gute Sache, denke ich. “(Matsuno)

\subsubsection{Wer wird Politiker? - Probleme bei der Kandidatenrekrutierung}

Die Forderung seitens der Interviewpartner von der LDP nach weiteren Reformen im Bereich der Kandidatenrekrutierung, entsprang nicht zuletzt ihrem Eigeninteresse. Einige befragte LDP-Abgeordnete äußerten die Befürchtung, dass die bisher praktizierte Kandidatenauswahl die Akzeptanz der Kandidaten in den Wahlkreisen und damit auch die Position der Partei schwächt. Der Abgeordnete Harada forderte in diesem Zusammenhang eine verstärkte Öffnung der Partei: „Die Bürger werden ein breiteres Auswahlverfahren fordern, und wenn man dann diese Vorwahlen durchführt, wird er [der Erbabgeordnete - d.A.] nicht zwangsläufig gewinnen. Die LDP selbst muss sich öffnen. Und damit sie regieren kann, muss auch die Zahl der Parteimitglieder steigen. Wenn die Partei sich den Mitgliedern nicht weiter öffnet, wird sie verlieren. “

Ein anderes Problem scheint die Abwanderung von Nachwuchskräften aus der LDP darzustellen. Alle LDP-Abgeordneten äußerten in den Interviews diese Sorge mit dem Hinweis darauf, dass es unter dem bisherigen System in ihrer Partei sehr schwierig sei, junge Talent, die über keine familiären Verbindungen in die Politik verfügen, zu fördern. Die bereits genannten Bestimmungen der LDP zur Kandidatenrekrutierung enthalten zwar einen Passus, nach dem die Kandidaturen von Personen aus den unterschiedlichsten Bereichen, insbesondere auch von Frauen, willkommen ist. ${ }^{476}$ Tatsächlich sind die Rekrutierungspfade vor allem in der LDP noch sehr geschlossen. Der Abgeordnete Itô war sogar der Meinung, dies habe sich nach Einführung des neuen Wahlsystems sogar noch verstärkt: „Es wäre gut, über ein System nachzudenken, in dem Kandidaten antreten könnten, deren Eltern nicht Abgeordnete waren. Das ist in den Einerwahlkreisen in der Realität sehr schwer.“

\footnotetext{
${ }^{476}$ Vgl.: Jiyûminshutô: Kôhosha sentei kijun, a.a.O.
} 
Auch Otake weist darauf hin, dass aus den oben genannten Gründen die Chancen für eine erfolgreiche Kandidatur für Nicht-Erbpolitiker unter dem neuen Wahlsystem abgenommen hätten. ${ }^{477}$ Das gilt vor allem für die LDP, deren Rekrutierungsmechanismen teilweise sehr intransparent sind. In der Folge kommt es zur Abwanderung von Nachwuchspolitikern, die bei den Liberaldemokraten für sich keine Zukunft sehen. „Kandidaten, die unter dem alten Wahlsystem für die LDP kandidieren würden, aber in den Einerwahlkreisen nicht als offizielle Kandidaten aufgestellt werden können, gehen im Endeffekt in die DPJ“, so Itô Shintarô.

Der Abgeordnete sprach von 30 bis 35 solcher Fälle, die es in der Vergangenheit gegeben habe. Auch die anderen befragten LDP-Abgeordneten scheinen erkantn zu haben, dass auf diese Weise die Qualität der Kandidaten auf Dauer nicht gewährleistet werden kann. Diese Abwanderung von Nachwuchskräften in die DPJ wurde auch von dem DPJ-Abgeordneten Matsuno bestätigt und damit erklärt, dass in der DPJ auch Kandidaten ohne eigene kôenkai eine Chance hätten. In der DPJ scheint somit die Unterstützung eines offiziellen Kandidaten durch die lokalen Parteiorganisationen besser zu funktionieren als bei den Liberaldemokraten. Diesen Nachteil für die LDP sah auch Itô: „Diese Leute müssten eigentlich in der LDP starten, gehen aber in die DPJ. In der DPJ, es ist ja eigentlich nicht gut, über eine andere Partei zu sprechen, aber dort gibt es Leute, die eigentlich eine ganz andere Einstellung haben. Aber um im Einerwahlkreis zu gewinnen, brauchen sie eine große Wählerbasis. Weil sie keine Unterstützung von der LDP bekommen können, treten sie für die DPJ an. “

Bei den Politikern, die in die DPJ gewechselt sind, handelt sich jedoch offensichtlich nicht nur um ,junge Talente“, die noch aufgebaut werden müssen. Der Abgeordnete Ôno dazu: „Die Ausübung einer Funktion in der Partei, die Tätigkeit in der Lokalpolitik oder die Erbfolge sind die drei Möglichkeiten, die als Voraussetzung für eine Kandidatur für die LDP gelten. Doch die Erbabgeordneten gewinnen meistens. Die Funktionsträger wechseln dann oft in die DPJ. Das macht mir ein wenig Angst. “ Dass dieser Aderlass langfristig zu einem Problem für die LDP werden wird, scheint in der LDP durchaus erkannt worden zu sein.

Auf diese Weise haben sich die neuen konservativen Parteien nicht nur zu einer Konkurrenz bei der Sammlung von Wirtschaftsspenden oder Stimmen aus dem konservativen Lager entwickelt, sondern treten auch bei der Rekrutierung von Nachwuchskräften in Wettbewerb mit der LDP. Gerade während der Übergangszeit, Mitte der 1990er Jahre, knüpften viele junge Politiker der LDP Kontakte zu den neuen konservativen Parteien. ${ }^{478}$ Tatsächlich war es unter dem 1955er-System fast undenkbar, dass beispielsweise Personen aus der Ministerialbürokratie in einer anderen Partei als der LDP eine politische Karriere begannen. Heute dagegen gebe es

\footnotetext{
${ }^{477}$ Vgl.: Otake, Hideo: A.a.O., S. 281.

${ }^{478}$ Vgl.: Jain, Purnendra C.: Party Politics at the Crossroads, a.a.O., S. 24.
} 
einige solcher Fälle, wie Itô Shintarô bemerkte. Unter diesem Gesichtpunkt sind also weitere innerparteiliche Veränderungen aus Sicht der Liberaldemokraten von größter Wichtigkeit.

Köllner weist allerdings darauf hin, dass es umgekehrt sehr schwierig für DPJ-Politiker oder unabhängige Kandidaten sei, in die LDP zu wechseln. Durch die Einführung der Einerwahlkreise, konzentriert sich die Partei vor allem auf ihre Mandatsträger und offiziellen Kandidaten vor Ort. ${ }^{479}$

Einige Interviewpartner, wie der Abgeordnete Harada, sahen bereits Anzeichen eines Wandels in der LDP: „Na ja, es gibt derzeit bei den Vorwahlen eine Menge Leute, die sagen ,ich kann das machen' und die auch gewählt werden können. Die eine Chance bei den Wahlen haben. Daran nehmen sehr viele Leute teil, nicht wahr. In der LDP findet ein Systemwandel statt, dass auch Leute teilnehmen können, die keine Erbfolge antreten. Die LDP öffnet sich. “

Generell wurden die Rekrutierungspfade in Japan aber von den Interviewpartnern, sowohl von den LDP-Politikern als auch von den DPJ-Politikern, als relativ geschlossen bezeichnet. Der Abgeordnete Terata wies in diesem Zusammenhang auf das Problem der sozialen Immobilität japanischer Politiker hin. Diese sei ein Grund für das hohe Durchschnittsalter japanischer Abgeordneter, die nach dem Ausscheiden aus der Politik selten die Möglichkeit haben, wieder in ihren alten Beruf zurückzukehren. Auffangpositionen sind wie bereits erwähnt nur in geringem Maße vorhanden. Ein Recht auf Urlaub während des Wahlkampfes, wie es in Deutschland (Art. 48 GG, Satz 1) existiert, gibt es in Japan nicht. Um mehr Menschen einen Weg in die Politik zu ermöglichen, müsse auch an dieser Stelle über Reformen nachgedacht werden, so der junge Abgeordnete, und forderte die Einführung eines „Politikurlaubs“.

Viele der befragten Abgeordneten sprachen generell von Problemen, junge Nachwuchskräfte zu finden, vor allem in ländlichen Gebieten. Dabei haben junge Kandidaten durchaus gute Chancen in den Wahlkämpfen zu gewinnen, wie Terata Manabu anmerkte: „Tatsächlich ist es so, dass die jungen Leute in den Wahlen sehr stark sind. Wenn gegen einen 70-jährigen Abgeordneten ein agiler 35-jähriger Kandidat antritt, hängen die Wähler ihre Hoffnungen an den 35-Jährigen.“

In der Realität sind die Zugangsmöglichkeiten in die nationale Politik jedoch immer noch sehr stark eingeschränkt und junge Interessenten ohne die genannten Ressourcen oder Verbindungen haben selten eine Chance auf eine offizielle Kandidatur. Deshalb waren sich alle Interviewpartner darüber einig, dass es bei der politischen Rekrutierung weitere Veränderungen geben muss. So wurde vor allem ein einheitliches Auswahlsystem (innerhalb der jeweiligen Partei) gefordert, in dem auch Kandidaten ohne familiäre Bindungen eine Chance haben. Die

\footnotetext{
${ }^{479}$ Vgl.: Köllner, Patrick: Zwischen Konflikt, Kohäsion und parteiübergreifender Kooperation: Probleme und Perspektiven der Demokratischen Partei Japans, a.a.O., S. 58.
} 
konkreten Vorschläge waren allerdings sehr unterschiedlich. Sie reichten von der einheitlichen Durchführung von (parteiinternen) Vorwahlen bis zur Wiedereinführung des alten Wahlsystems, das ihrer Meinung nach mehr „Newcomern“ die Möglichkeit bot, zu kandidieren.

In der LDP haben sich in den vergangenen Jahren mehrere Kommissionen mit einer Reform der Kandidatenbestimmung beschäftigt. Der LDP-Ausschuss für Wahlangelegenheiten legte 2003 ein Konzeptpapier vor, nach dem es keine Bevorzung der Amtsinhaber mehr geben sollte. Stattdessen wurde vorgesehen, die Kandidaten der Einerwahlkreise durch Vorwahlen zu bestimmen, um die Strukturen transparenter zu machen. Wer zweimal in Folge in seinem Wahlkreis verloren hat, soll nicht mehr antreten dürfen. Neben den vorgesehenen Vorwahlen, die aufgrund der Mitgliederstrukturen und der wenigen aktiven Parteimitglieder als wenig sinnvoll betrachtet wurden, rief vor allem eine geplante Altersbeschränkung für die Kandidaten auf der Parteiliste Kritik hervor. ${ }^{480}$ Inzwischen wurde aber eine Altersbeschränkung für eine Kandidatur auf der Parteiliste eingeführt, die bei 73 Jahren liegt. ${ }^{481}$

Die Vorschläge des Ausschusses zur Untersuchung und Förderung von Parteireformen in der LDP (Jimintô kaikaku kenshô/suishin iinkai) unter dem ehemaligen Parteisekretär Abe Shinzô gehen in die gleiche Richtung. Auch hier werden öffentliche Auswahlsysteme gefordert, die auf die gesamte Partei angewendet werden sollen. Die Vorschläge des Ausschusses wurden bisher aber mit Ablehnung aufgenommen, vor allem wegen der darin enthaltenen Pläne zu einer Neuordnung der Finanzflüsse in der Partei. ${ }^{482}$

Obwohl sich an einigen Stellen das starre Rekrutierungssystem schon geöffnet hat, besteht also die Notwendigkeit weiterer Reformen der innerparteilichen Rekrutierungsmechanismen. Bereits existierende konkrete Vorschläge wurden jedoch bisher abgelehnt. Es ist aber auch schwer vorstellbar, dass die Mandatsträger für sie nachteilige Bestimmungen, wie etwa die Aufhebung der automatischen Nominierung, einführen. ${ }^{483}$ Interviewpartner wie Ishiba Shigeru, die sich bisher sehr stark für Reformen (s.o.) eingesetzt haben, zeigten sich etwas enttäuscht über den bisher verlaufenen Prozess.

An dieser Stelle sollte man anfügen, dass die Erbfolge als solche von den meisten Interviewpartnern nicht als grundsätzlich negativ bewertet wurde. Eigenschaften wie Pflichtgefühl oder

\footnotetext{
${ }^{480}$ Vgl.: Japan aktuell: Gerangel in der LDP um Parlamentskandidaturmodi geht weiter, a.a.O., S. 147.

${ }^{481}$ Vgl.: Asahi Shinbun: Hashimoto gen-shushô, intai mitooshi, in: Asashi Shinbun, 12.08.2005, S. 1. (Asahi Shinbun: Rücktritt des ehemaligen Ministerpräsidenten Hashimoto absehbar.)

${ }^{482}$ Vgl.: Japan aktuell: Abe als Parteireformer in der LDP? In: Japan aktuell. Wirtschaft. Politik. Gesellschaft, Heft Oktober/2004, S. 379f; Japan aktuell: Geplante institutionelle Veränderungen in der LDP: Entscheidungsprozesse und Kandidatenauswahl, in: Japan aktuell. Wirtschaft. Politik. Gesellschaft, Heft Februar/2004, S. 33f.

${ }^{483}$ Vgl.: Japan aktuell: Geplante institutionelle Veränderungen in der LDP: Entscheidungsprozesse und Kandidatenauswahl, in: Japan aktuell. Wirtschaft. Politik. Gesellschaft, Heft Februar/2004, S. 33f.
} 
politische Erfahrungen durch das Aufwachsen in einer Politikerfamilie wurden als positive Seiten der Erbabgeordneten betrachtet. Der Abgeordnete Harada: „Auf der einen Seite ist es gut, dass sie als Sekretär oder an Aktivitäten bei ihrem Vater mitgearbeitet haben. Diese Erfahrungen sind nützlich für die Politik. Das ist ein Punkt von vielen. Deshalb gibt es Fälle, bei denen es positiv bewertet wird, und Fälle, bei denen es negativ bewertet wird. Es geht natürlich nicht, dass nur die Erbfolge zählt. Kurz gesagt gibt es positive und negative Bewertungen. Es gibt gute und schlechte Seiten der Erbfolge. Jetzt kommt aber die Zeit, in der nach Offenheit und Transparenz bewertet wird. “ 


\subsection{Die Wahlen 2005: Trends in der Kandidatenaufstellung}

Als ein geplantes Gesetz zur Postreform im japanischen Oberhaus mit Stimmen aus seiner eigenen Partei zu Fall gebracht wurde, löste Ministerpräsident Koizumi Junichirô das Unterhaus auf und setzte für den 11. September 2005 Neuwahlen an. Bereits bei der Abstimmung im Unterhaus hatten zahlreiche LDP-Abgeordnete gegen die geplante Privatisierung der Post gestimmt. Die vorliegende Arbeit wurde zwar im Wesentlichen vor den Wahlen fertig gestellt, so dass eine Analyse der Wahl unter dem Gesichtspunkt der politischen Rekrutierung nicht mehr möglich war, dennoch sollen einige Trends in der Kandidatenaufstellung genannt werden.

Die geplante Privatisierung der japanischen Post gilt als eine der wichtigsten Reformmaßnahmen des Ministerpräsidenten Koizumi, ist aber gerade unter konservativen Politikern umstritten. Die rund 400.000 Postangestellten gelten als zuverlässige Wählerbasis der LDP und stellen gerade in ländlichen Regionen wichtige Multiplikatoren dar. Darüber hinaus verfügt die Postbank über Einlagen von umgerechnet 2,5 Billionen Euro, die häufig zur Finanzierung verschiedener staatlicher Investitionsvorhaben eingesetzt werden. Die Abstimmungsniederlage kam zwar nicht überraschend, sie ist aber auch als Beleg des Streits zwischen Koizumi und der alten Garde der LDP zu bewerten. So war die Kamei-ha an der Abstimmungsniederlage maßgeblich beteiligt.

Zunächst verkündete die LDP, Abgeordnete, die gegen die Postreform gestimmt haben, sich enthalten haben oder bei der Abstimmung abwesend gewesen waren, nicht mehr aufzustellen. Diesen Politikern blieb nur eine unabhängige Kandidatur oder der Wechsel in eine andere Partei. $^{484}$

Dies ist ein Indiz dafür, dass die Parteizentrale der LDP sich wohl auf diese Weise gleichzeitig mehr Einfluss bei der Kandidatenbestimmung verschaffen möchte. Es bleibt allerdings abzuwarten, wie sich diese Bestimmung auf die innerparteilichen Machtverhältnisse auswirkt. Als Druckmittel scheint diese Maßnahme schon zu wirken. Der Abgeordnete Yoshida trat vor der Wahl aus der Kamei-ha aus. ${ }^{485}$ Der Faktionsführer Kamei gab indes die Gründung einer neuen Partei, der Kôkuminshintô (Neue Volkspartei), bekannt. ${ }^{486}$ Außerdem trat die neu gegründete Nihon Shintô an.

\footnotetext{
${ }^{484}$ Vgl.: Yomiuri Shinbun: Jimin, 1-ji kônin-kôho 170-nin-o hyômei, http://www.yomiuri.co.jp/politics/news/20050813it13.htm, Download vom 13.08.2005. (Die Liberaldemokraten stellen die ersten 170 offiziellen Kandidaten vor.)

${ }^{485}$ Vgl.: Yomiuri Shinbun: Jimin-no Yoshida Rokuzaemon-shi, Kamei-ha-o ridatsu, http://www.yomiuri.co.jp/politics/news/20050812ia24.htm, Download vom 13.08.2005. (Yoshida Rokuzaemon von der LDP verlässt die Kamei-ha.)

${ }^{486}$ Vgl.: Yomiuri Shinbun: „kokuminshintô“ 5 nin-de kessei, in Yomiuri Shinbun, 18.08.2005, S. 1. (Yomiuri Shinbun: „Kokuminshintô“ von fünf Personen gegründet.)
} 
An der Kandidatenaufstellung scheint sich noch nicht allzu viel geändert zu haben. In der LDP erfolgte die Aufstellung bisher auf der Grundlage „informeller“ Regelungen. Amtsinhaber und bisherige Kandidaten behielten, mit Ausnahme der oben genannten Widerständler gegen die Postreform, ihren Wahlkreis. Unter den Kandidaten der LDP befanden sich 62 „Newcomer“. 60 Wahlkreise galten als „vakant“, in 15 davon gab es „öffentliche Aufrufe“, um die Kandidaten öffentlich auszuwählen. ${ }^{487}$

Die DPJ stellte in 290 von 300 Direktwahlkreisen einen Kandidaten auf. Auch hier konnten 172 der 176 Abgeordneten ihren Wahlkreis behalten. Außerdem wurden 13 ehemalige Abgeordnete aufgestellt, die in der letzten Wahl kein Mandat erhalten hatten. Die Zahl der neuen Kandidaten lag mit 88 Personen über der Zahl der LDP. Unter den Kandidaten befanden sich auch 18 Frauen. Die Zahl der Wahlkreise, in denen die Kandidaten öffentlich nach Ausschreibungen bestimmt wurden, lag mit 18 Bezirken jedoch kaum höher als bei der LDP. ${ }^{488}$ Diese Tatsache konterkariert jedoch das Selbstbild der DPJ, die sich ja in Bezug auf die Kandidatenrekrutierung gern als transparentere Partei darstellt.

Abbildung 23: Ergebnis der Unterhauswahl 2005

\begin{tabular}{l||c|c|c|c|c|c|c|c||c} 
Partei & LDP & DPJ & CGP & KPJ & SDPJ & $\begin{array}{c}\text { Nihon } \\
\text { Shintô }\end{array}$ & $\begin{array}{c}\text { Kokumin } \\
\text { Shintô }\end{array}$ & $\begin{array}{c}\text { Unabh./ } \\
\text { Sonstige }\end{array}$ & Gesamt \\
\hline \hline Direktmandat & 219 & 52 & 8 & - & 1 & - & 2 & 18 & $\mathbf{3 0 0}$ \\
\hline Listenplatz & 77 & 61 & 23 & 9 & 6 & 1 & 2 & 1 & $\mathbf{1 8 0}$ \\
\hline \hline & $\mathbf{2 9 6}$ & $\mathbf{1 1 3}$ & $\mathbf{3 1}$ & $\mathbf{9}$ & $\mathbf{7}$ & $\mathbf{1}$ & $\mathbf{4}$ & $\mathbf{1 9}$ & \\
Gesamt & $(237)$ & $(177)$ & $(34)$ & $(9)$ & $(6)$ & $(-)$ & $(-)$ & $(17)$ & $\mathbf{4 8 0}$
\end{tabular}

Quelle: Asahi Shinbun: Yotô 327, shûin-no 2/3 okosu, in: Asahi Shinbun, 12.09.2005, S. 1. (Asahi Shinbun: 327 [Mandate] für die Regierungsparteien - 2/3-Mehrheit im Unterhaus .)

Die Erfahrungen, die die LDP mit den öffentlichen Auswahlverfahren bei dieser Wahl machte, scheinen sehr positiv zu sein. Die Kandidatenaufstellung wurde in diesen Fällen als gut

\footnotetext{
${ }^{487}$ Vgl.: Yomiuri Shinbun: Shûinsen-no kôhoyôritsu-rasshu, jimin-ha kenkyû-kôbo, http://www.yomiuri.co.jp/politics/news/20050813it01.htm, Download vom 13.08.2005. (Vorstoß bei der Unterstützung der Kandidaten, notwendige öffentliche Ausschreibungen bei der LDP) ${ }^{488}$ Vgl.: Ebd.
} 
nachvollziehbar gelobt und die Partei konnte einige der betroffenen Wahlkreise nach langer Zeit wieder zurückerobern. ${ }^{489}$

Dennoch scheint sich die Erbfolge als Rekrutierungspfad weiterhin behaupten zu können, sowohl in der LDP als auch in der DPJ. Unter den aufgestellten Kandidaten waren 158 Erbpolitiker, das entspricht einem Anteil von 14\% an der Gesamtzahl der Kandidaten. ${ }^{490}$

Einige Erbfolgen sorgten bereits für Schlagzeilen. Zwei ehemalige Parteigrößen der LDP, der frühere Ministerpräsident Hashimoto Ryûtarô und Mutô Kabun kündigten bereits an, nicht mehr zu kandidieren und stattdessen ihre Söhne aufstellen zu wollen. Gerade die Erbfolge des Sohnes von Hashimoto Ryûtarô, der während seiner politischen Laufbahn in zahlreiche Skandale verstrickt war, rief Kritik hervor. Allerdings wurde die Kandidatur seines 31-jährigen Sohnes nur möglich, weil eine weitere potenzielle Kandidatin verzichtete. Die kôenkai von Hashimoto Ryûtarô favorisierte nämlich seine Ehefrau und versuchte vergeblich, sie zu einer Kandidatur zu bewegen. ${ }^{491}$ Hashimoto Gaku konnte in der Wahl zwar kein Direktmandat erringen, ist aber über die Parteiliste ins Unterhaus eingezogen.

\footnotetext{
${ }^{489}$ Vgl.: Asahi Shinbun: Kôbo. Kihara-shi, sessen seisu, in: Asahi Shinbun, 12.09.2005, S. 1. (Asahi Shinbun: Öffentliche Ausschreibung: Kihara kann Kopf-an-Kopf-Rennen für sich entscheiden .)

${ }^{490}$ Vgl.: Johnston, Eric: Family-bred politicians fan out, in: The Japan Times Online, http://www.japantimes.com/cgi-bin/getarticle.pl5?nn20050910f1.htm, Download vom 12.09.2005.

${ }^{491}$ Vgl.: Yomiuri Shinbun: Hashimoto gen-shushô-no kôkei jinan yôritsu, okayama 4 ku-kara shutsuba, http://www.yomiuri.co.jp/politics/news/20050812ia24.htm, Download vom 13.08.2005.

(Unterstützung der Kandidatur des jüngeren Sohnes vom ehemaligen Ministerpräsidenten Hashimoto, er startet inOkayama 4.); Asahi Shinbun: Hashimoto gen-shushô, intai mitooshi, a.a.O., S. 1.
} 


\section{Konklusion}

Ziel der Arbeit war es, die Entstehung dieses Rekrutierungspfades in der japanischen Politik zu erklären und die Auswirkungen der politischen Reformen Mitte der 1990er Jahre auf die Erbfolge zu analysieren. Dabei konzentrierte sich die Analyse auf die Unterhausabgeordneten der LDP und der DPJ. Das Rekrutierungsmodell nach Norris und Lovenduski, das auf die vorliegende Untersuchung angewendet wurde, hat sich als sinnvoller Rahmen zur Strukturierung der Analyse erwiesen.

Die Erbfolge wird häufig als typisch japanisches Phänomen betrachtet. ${ }^{492}$ Dieser Karrierepfad ist, wie im Rahmen dieser Arbeit gezeigt wurde, eng mit den politischen Rahmenbedingungen unter dem so genannten 1955er-System und mit der größten japanischen Partei, der LDP, verbunden. Die Opportunitätsstruktur jener Zeit führte dazu, dass sowohl auf der Nachfrageseite, der Auswahl seitens der Parteien und der Wahlkreisorganisationen, als auch auf der Angebotsseite, den vorhandenen Ressourcen der Aspiranten, Erbpolitiker über Vorteile gegenüber anderen Kandidaten verfügten, die keine familiären Verbindungen in die Politik hatten.

Das Wahlsystem der Mehrerwahlkreise, in denen auch Kandidaten derselben Partei gegeneinander antraten, förderte den Aufbau von Wahlkampforganisationen, die an die Person des Kandidaten gebunden waren, und schwächte die offiziellen Parteiorganisationen. Das galt vor allem für die bis 1993 regierende Liberaldemokratische Partei, die darüber hinaus in mehrere Faktionen, die habatsu, gespalten war. Sowohl die Bedeutung dieser persönlichen Unterstützerorganisationen als auch der Einfluss der habatsu bei der Kandidatenrekrutierung führten dazu, dass eine LDP-Kandidatur nur einem kleinen Personenkreis offen stand. Nicht nur bestimmte Berufsgruppen, namentlich Politiker aus der Regionalpolitik, Sekretäre von Abgeordeten und ehemalige Ministerialbeamte, dominierten unter den Abgeordneten der Liberaldemokraten. Bei der Auswahl von Kandidaten spielten vor allem familiäre Verbindungen eine große Rolle.

Im Wahlkreis stellten die persönlichen Unterstützerorganisationen eine Art gatekeeper dar. Sie entschieden durch ihre Gefolgschaft häufig über die Aufstellung eines Kandidaten. Da die kôenkai an einer erfolgreichen Weiterführung ihrer Beziehungen zum Abgeordneten interessiert waren, wurde ein Nachfolger nicht selten im engeren Familienkreis gesucht. Die Anerkennung als offizieller Kandidat erfolgte zwar auf zentraler Ebene durch die Partei. Hier lag die Entscheidung aber zumeist bei den habatsu, die ein Interesse daran hatten, den Nachfolger einer ,ihrer“ Abgeordneten an sich zu binden. Eine Nachfolgeregelung innerhalb der Familie war

${ }^{492}$ Vgl.: Masayama, Mikitaka: A.a.O., S. 49. 
deshalb willkommen, um die Loyalitätsverhältnisse weiterzuführen. Auf der Angebotsseite verfügten Kandidaten, die eine Erbfolge antraten, neben einer zumeist erfahrenen Wahlkreisorganisation über einen bekannten Namen, der in den personalisierten Wahlkämpfen von großer Bedeutung war. Daneben war es für Erbpolitiker häufig einfacher, aufgrund der Netzwerke ihrer Vorgänger finanzielle Ressourcen zu mobilisieren. Mit diesen Ressourcen ausgestattet, bestand für Erbpolitiker immer noch die Möglichkeit, als unabhängiger Kandidat anzutreten und nachträglich in die Partei aufgenommen zu werden.

Man muss allerdings einschränken, dass die genannten Vorteile, mit Ausnahme des bekannten Namens, für alle Kandidaten galten, die irgendeine Form der Nachfolge antraten, sei es ein Familienmitglied oder ein ehemaliger Sekretär des Abgeordneten. Jedoch war die Wahrscheinlichkeit, als Kandidat von der kôenkai ausgesucht zu werden, für Familienmitglieder relativ hoch. $^{493}$

Dass dieses System der politischen Rekrutierung von den Wählern getragen wurde, lässt sich wiederum mit dem Wahlsystem, mit einem ausgeprägten Klientelismus und Patronage erklären. Auch einige historisch-kulturelle Faktoren, etwa die japanische Familienstruktur, begünstigten die Übertragung eines gewachsenen Loyalitätsverhältnisses vom Abgeordneten auf seinen Nachfolger. Dieses System des Klientelismus und der Patronage ließ sich aber nur so lange aufrecht erhalten, wie die LDP-Abgeordneten als Angehörige der Regierungspartei entsprechende Ressourcen erhielten und an ihre Wählerschaft verteilen konnten.

Der Zusammenbruch des 1955er-Systems und die geplanten Reformen weckten nicht zuletzt die Hoffnung auf mehr Offenheit und Transparenz sowohl bei der Kandidatenauswahl als auch in den Wahlkämpfen selbst. Als Folge der Reformen, die vor allem das Wahlsystem und die Parteienfinanzierung betrafen, erhoffte man eine Stärkung der lokalen Parteiorganisationen gegenüber den persönlichen Unterstützerorganisationen und ein Ende der money-powered politics. Auf diese Weise sollten Bedingungen geschaffen werden, unter denen eine Kandidatur einem breiteren Personenkreis offen steht. Diese Hoffnungen haben sich bisher im Großen und Ganzen nicht erfüllt.

Da es zu den Auswirkungen der politischen Reformen auf die Erbfolge in der japanischen Politik insgesamt nur recht wenig Literatur gibt, baute der zweite Teil der vorliegenden Arbeit vor allem auf qualitativen Interviews auf. Die Analyse der Interviews, aber auch die vorhandene Literatur zeigen, dass sich die Rekrutierungspfade in der japanischen Politik zwar leicht geöffnet haben und Kandidaten ohne entsprechenden familiären Hintergrund vermehrt Chan-

\footnotetext{
${ }^{493}$ Vgl.: Ishibashi, Michihiro; Reed, Steven R.: A.a.O., S. 373.
} 
cen für eine Kandidatur erhalten. Generell konnte sich der Rekrutierungspfad des Erbabgeordneten aber bisher behaupten und wird wohl auch weiterhin eine große Rolle in der japanischen Politik spielen.

Auf der Nachfrageseite, bei der Auswahl der Kandidaten in den Parteien, ist es weniger zu grundlegenden Veränderungen als vielmehr zu Machtverschiebungen gekommen. Während in der LDP die habatsu offensichtlich an Einfluss verloren haben, hat die Parteizentrale bei der Kandidatenaufstellung deutlich mehr Macht als noch vor zehn Jahren. Auch im Zusammenhang mit den neu eingeführten Parteilisten, kann man von Zentralisierungstendenzen sprechen. Das heißt aber nicht zwangsläufig, dass sich die Chancen von Erbpolitikern, eine offizielle Kandidatur zu erhalten, verschlechtert haben. Gerade in der LDP haben sich häufig eingeübte Verhaltensmuster bei der Kandidatenauswahl erhalten.

Verstärkend wirkt, dass es die Partei bisher nicht geschafft hat, einheitliche Auswahlmechanismen durchzusetzen. Die kôenkai sind in vielen Wahlkreisen immer noch die Selektoren, die eine Nachfolge der Unterstützerorganisation mitentscheiden. Auch von der Stärkung der Parteien im Zuge der politischen Reformen hat man sich offensichtlich zu viel versprochen. In vielen konservativen Parteien sind für eine erfolgreiche offizielle Nominierung die informellen Netzwerke in den Parteien immer noch bedeutender als die zumeist recht schmale Parteibasis in den Wahlkreisen. Allerdings, so ist nicht zuletzt in den geführten Interviews deutlich geworden, kann sich die Parteizentrale bei Konflikten verstärkt durchsetzen. Die Rekrutierungswege haben sich durchaus leicht geöffnet.

Auch auf der Angebotsseite verfügen die Erbabgeordneten mit ihren Ressourcen über einen Vorsprung vor ihren Konkurrenten, da sich die Verhältnisse in den Wahlkreisen zumindest in den ländlichen Regionen nicht grundlegend geändert haben. Die persönliche Unterstützerorganisationen sind immer noch die Basis konservativer Kandidaten, deren Wahlkämpfe weiterhin durch Personalisierung und Klientelismus geprägt sind. Interessant ist dabei der Hinweis vieler Interviewpartner auf so genannte soft skills, über die Erbabgeordnete durch das Aufwachsen in einer Politikerfamilie verfügen. Diese Fähigkeiten, auch die Motivation und das vorhandene Interesse an Politik sollten nicht unterschätzt werden.

In der LDP sind die Chancen für Kandidaten, die nicht über entsprechende persönliche Unterstützergruppen und die finanziellen Mittel verfügen, gering. Eine Stärkung der lokalen Parteiorganisationen und damit eine aktivere Teilnahme der Parteimitglieder, eines der Reformziele, ist bisher noch nicht in dem erhofften Ausmaß erfolgt. In den Augen einiger der befragten Abgeordneten hat die Einführung der Einerwahlkreise sogar dazu geführt, dass die Chancen für Erbabgeordnete gestiegen sind. Für einen Herausforderer aus der Partei ist es demnach schwie- 
riger geworden, sich gegen den Abgeordneten des Wahlkreises oder dessen designierten Nachfolger durchzusetzen, da nur noch ein Kandidat pro Partei aufgestellt wird.

Die Veränderungen in der Opportunitätsstruktur und auf der Nachfrageseite haben also nicht ausgereicht, die nationale Politik einem größeren Personenkreis zu öffnen und die Chancen anderer Kandidaten denen der Erbabgeordneten anzugleichen. Das lässt sich zum einen mit unvollständigen Reformen, vor allem uneinheitlichen Regelungen innerhalb der Parteien, zum anderen mit der nach wie vor großen Ressourcenausstattung auf der Angebotsseite erklären. Die Chancen für Kandidaten ohne familiäre Bindungen innerhalb der DPJ, und in wohl geringem Maß in der LDP, sind zwar teilweise gestiegen, die Erbfolge wird in der japanischen Politik aber mittelfristig eine Zukunft haben, auch wenn dabei zwischen der LDP und anderen konservativen Oppositionsparteien differenziert werden muss. Diese Aussagen über die Zukunft der Erbfolge kann man aufgrund der im zweiten Teil vorgestellten qualitativen Interviews mit japanischen Unterhausabgeordneten treffen. Es sind allerdings Tendenzen sichtbar, die Auswahlmodi zu vereinheitlichen und transparenter zu gestalten, was langfristig die Vorteile von Erbpolitikern reduziert.

Vorreiter auf diesem Gebiet ist sicherlich die DPJ, die verstärkt zu Vorwahlen und öffentlichen Auswahlsystemen übergegangen ist. Allerdings entspricht hier das dargestellte Selbstbild einer für einen großen Personenkreis offenen und transparenten Partei nicht immer der Realität. Inwieweit das Image einer Reformpartei, vor allem nach der geplanten Fusion mit der Liberalen Partei, gerechtfertigt ist, und wie stabil die aus verschiedenen politischen Strömungen bestehende Partei in Zukunft sein wird, ist eine andere Frage. Fest steht, dass die Chancen für Kandidaten, die keine Erbabgeordneten sind, größer sind, sowohl eine Kandidatur als auch Unterstützung durch die Partei im Wahlkampf zu bekommen. Angesichts transparenterer Auswahlverfahren in der DPJ erweisen sich die Defizite bei der Kandidatenauswahl zunehmend als problematisch für LDP. Wie die Interviewpartner anmerkten, tritt die DPJ immer stärker in Konkurrenz mit der LDP um geeignete Nachwuchskräfte. Die Oppositionspartei ist vor allem für Politiker attraktiv, die sich bei den Liberaldemokraten beim Kampf um eine offizielle Kandidatur nicht gegen die Erbpolitiker durchsetzen können.

Dieses Problem hat man auch in der LDP erkannt, so dass auf lange Sicht wohl kein Weg daran vorbeiführt, die Kandidatenrekrutierung transparenter und von einer Erbfolge unabhängiger zu machen. Zumal die Erfahrungen der vergangenen Wahl 2005 in den Wahlkreisen, in denen öffentliche Auswahlverfahren durchgeführt wurden, durchaus positiv waren, sowohl was die Qualität der aufgestellten Kandidaten als auch die Unterstützung seitens der Wählerschaft 
betraf. Ansätze seitens verschiedener Reformkommissionen, z.B. unter Abe Shinzô, eine einheitliche und transparente Kandidatenauswahl einzuführen, sind zwar erkennbar, konnten sich jedoch noch nicht vollständig durchsetzen. Da die Notwendigkeit zu transparenteren und offeneren Auswahlverfahren nicht zuletzt einem Eigeninteresse der Partei entspringt, wird es hier jedoch langfristig Veränderungen geben. 


\section{Anhang}

\subsection{Tabellen- und Abbildungsverzeichnis}

Abbildung 1: Typen politischer Rekrutierung …............................................................. 19

Abbildung 2: Rekrutierungsmodell nach Norris ................................................................ 20

Abbildung 3: Liste der befragten Abgeordneten.................................................................. 27

Abbildung 4: Wahlkreise der befragten Abgeordneten...................................................... 28

Abbildung 5: Japanische Parteien und organisierte Interessen ............................................. 36

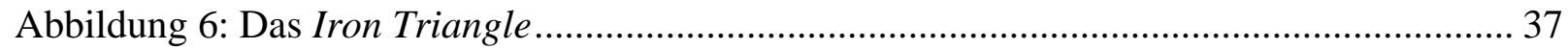

Abbildung 7: Karrierepfade ehemaliger Ministerialbeamter ............................................... 57

Abbildung 8: Japanische Erbabgeordnete 1994 nach Verwandtschaftsgrad ........................... 63

Abbildung 9: Politikerfamilien 1980/1990 nach Parteizugehörigkeit..................................... 66

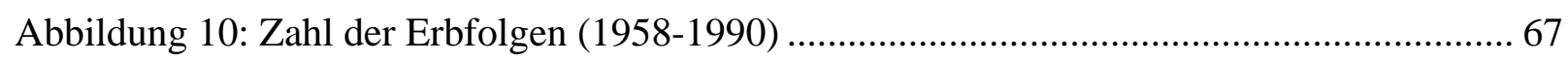

Abbildung 11: Japanische Erbabgeordnete 1990 nach Generation......................................... 68

Abbildung 12: Entwicklung der japanischen Parteien ab 1992 (vereinfacht)........................... 96

Abbildung 13: Die neuen Bestimmungen zur Politikfinanzierung ........................................ 106

Abbildung 14: Erbabgeordnete nach der Wahl 1994 (40. Legislaturperiode) ......................... 117

Abbildung 15: Erbabgeordnete nach der Wahl 2000 (42. Legislaturperiode) ........................ 117

Abbildung 16: Ergebnis der Unterhauswahl 2003 (aktuelle Sitzverteilung 2004) ................. 118

Abbildung 17: Erbabgeordnete nach der Wahl 2003 (43. Legislaturperiode) ........................ 119

Abbildung 18: Erbabgeordnete nach Direktmandat/Listenplatz ........................................... 124

Abbildung 19: Faktionszugehörigkeit der Interviewpartner (LDP) ...................................... 130

Abbildung 20: Neu gewählte Erbabgeordnete 2003 (43. Legislaturperiode) .......................... 161

Abbildung 21: Neu gewählte Erbabgeordnete 1993-2003 .................................................... 161

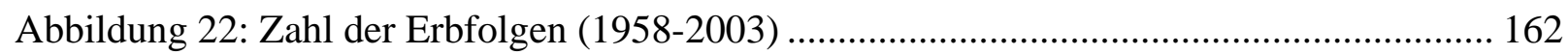

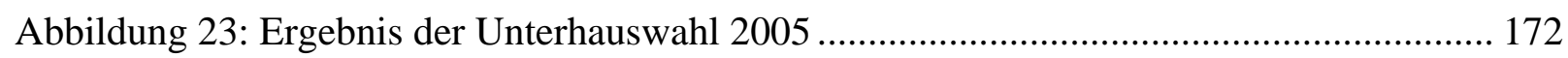

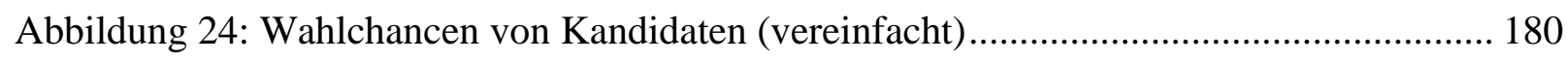

Abbildung 25: Berufe von japanischen Abgeordneten vor ihrer Wahl 1990......................... 180

Abbildung 26: Berufe von japanischen Abgeordneten vor ihrer Wahl 1996.......................... 181

Abbildung 27: Japanische Unterhausabgeordnete: Bildungsstrukturen................................. 181 


\subsection{Daten zu japanischen Abgeordneten}

\section{Abbildung 24: Wahlchancen von Kandidaten (vereinfacht)}

\begin{tabular}{l||c|c|c|c}
\multicolumn{1}{c|}{ Land } & Kandidaten & Abgeordnete & Gewählt (\%) & Jahr \\
\hline \hline \multicolumn{1}{l||}{ Schweden } & 5256 & 349 & 6,6 & 1988 \\
\hline Italien & 8631 & 630 & 7,3 & 1987 \\
\hline Schweiz & 2411 & 200 & 8,3 & 1987 \\
\hline Belgien & 2114 & 212 & 10,0 & 1987 \\
\hline Kanada & 1574 & 295 & 18,7 & 1988 \\
\hline Frankreich & 1788 & 577 & 20,7 & 1988 \\
\hline Spanien & 1663 & 350 & 21,0 & 1989 \\
\hline Großbritannien & 2946 & 651 & 221 & 1991 \\
\hline Dänemark & 606 & 179 & 29,5 & 1990 \\
\hline USA & 836 & 435 & 52,0 & 1992 \\
\hline Japan & $\mathbf{9 5 3}$ & $\mathbf{5 1 2}$ & $\mathbf{5 3 , 7}$ & $\mathbf{1 9 9 0}$ \\
\hline
\end{tabular}

Nach: Norris, Pippa: Through the Eye of the Needle: Comparative Political Recruitment in Western Democracies, Unveröff. Mskr., APSA, Washington 1993, S. 29.

\section{Abbildung 25: Berufe von japanischen Abgeordneten vor ihrer Wahl 1990}

\begin{tabular}{|c|c|c|c|c|c|c|c|c|c|}
\hline & LDP & SPJ & Kômeitô & $\mathrm{KPJ}$ & DSP & Shaminren & Shinpô & Parteilos & Gesamt \\
\hline Ministerialbürokratie & 66 & 1 & 1 & $\mathbf{0}$ & $\mathbf{0}$ & 0 & $\mathbf{0}$ & 4 & 72 \\
\hline Lokale Beamte & 4 & 0 & 0 & 0 & 0 & 0 & 0 & 0 & 4 \\
\hline Öffentlicher Dienst & 0 & 2 & 2 & 0 & 0 & 0 & 0 & 0 & 4 \\
\hline Gouverneure & 2 & 0 & 0 & 0 & 0 & 0 & 0 & 0 & 2 \\
\hline Regionalparlament & 66 & 45 & 8 & 6 & 3 & 1 & $\mathbf{0}$ & 7 & 136 \\
\hline Bürgermeister & 8 & 5 & 0 & 0 & 1 & 0 & 0 & 0 & 14 \\
\hline Lokalpolitiker & 3 & 3 & 3 & 0 & 1 & 0 & 0 & 1 & 11 \\
\hline Sekretäre v. Abgeordneten & 67 & 4 & 2 & $\mathbf{0}$ & 4 & 1 & $\mathbf{0}$ & 1 & 79 \\
\hline Parteifunktionäre / Mitarbeiter & 1 & 7 & 9 & 1 & 3 & 0 & 0 & 0 & 21 \\
\hline Gewerkschaften & $\mathbf{0}$ & 41 & $\mathbf{0}$ & 4 & 2 & $\mathbf{0}$ & $\mathbf{0}$ & 2 & 49 \\
\hline Universitätsdozenten & 3 & 5 & 1 & 0 & 0 & 0 & 0 & 0 & 9 \\
\hline Kultur & 1 & 1 & 0 & 0 & 0 & 0 & 0 & 0 & 2 \\
\hline Medienberufe & 6 & 2 & 0 & 0 & 0 & 0 & 1 & 1 & 10 \\
\hline tarento („Prominente“) & 0 & 0 & 0 & 0 & 0 & 0 & 0 & 0 & 0 \\
\hline Justiz & 0 & 0 & 0 & 0 & 0 & 0 & 0 & 0 & 0 \\
\hline Anwälte & 5 & 11 & 7 & 4 & 0 & 1 & 0 & 0 & 28 \\
\hline Lehrer & 0 & 1 & 1 & 1 & 0 & 0 & 0 & 2 & 5 \\
\hline Ärzte & 1 & 2 & 1 & 0 & 0 & 0 & 0 & 0 & 4 \\
\hline Selbstverteidigungskräfte & 0 & 0 & 0 & 0 & 0 & 0 & 0 & 0 & 0 \\
\hline Angestellte & 13 & 1 & 0 & 0 & 0 & 0 & 0 & 1 & 15 \\
\hline Gewerbetreibende & 13 & 0 & 0 & 0 & 0 & 0 & 0 & 0 & 13 \\
\hline $\begin{array}{l}\text { Funktionäre in Interessen- } \\
\text { gruppen, Genossenschaften }\end{array}$ & 4 & 4 & 6 & 0 & 0 & 0 & 0 & 2 & 16 \\
\hline Unternehmer & 4 & 0 & 0 & 0 & 0 & 0 & 0 & 0 & 4 \\
\hline Freie Berufe, Selbständige & 0 & 0 & 1 & 0 & 0 & 1 & 0 & 0 & 2 \\
\hline Andere & 8 & 1 & 3 & 0 & 0 & 0 & 0 & 0 & 12 \\
\hline Sitze im Parlament & 275 & 136 & 45 & 16 & 14 & 4 & 1 & 21 & 512 \\
\hline
\end{tabular}

Nach: Yomiuri Shinbun: Gekihen-no seijisentaku. 89 san 90 shûgiinsen tettei bunseki, Yomiuri Shinbun-sha, Tôkyô o.J., S. 204. (Yomiuri Shinbun: Politische Auswahl im Umbruch. Analyse der Wahlen zum Oberhaus 1989 und zum Unterhaus 1990.) 
Abbildung 26: Berufe von japanischen Abgeordneten vor ihrer Wahl 1996

\begin{tabular}{|c|c|c|c|c|c|c|c|c|c|}
\hline & LDP & SDPJ & KPJ & DPJ & NFP & NPS & Minkairen & Parteilos & Gesamt \\
\hline Beamte $^{494}$ & 49 & $\mathbf{0}$ & $\mathbf{0}$ & 2 & 22 & $\mathbf{0}$ & 0 & 1 & 74 \\
\hline $\begin{array}{l}\text { Lokale Selbstverwatungsor- } \\
\text { gane }\end{array}$ & 1 & 0 & 0 & 0 & 2 & 0 & 0 & 0 & 3 \\
\hline Gouverneure & 2 & 0 & 0 & 1 & 1 & 1 & 0 & 0 & 5 \\
\hline Regionalparlament & 64 & 1 & 5 & 7 & 31 & $\mathbf{0}$ & $\mathbf{0}$ & 3 & 111 \\
\hline Bürgermeister & 6 & 1 & 0 & 2 & 5 & 0 & 0 & 0 & 14 \\
\hline Lokalpolitiker & 9 & 0 & 2 & 1 & 4 & 0 & 0 & 0 & 16 \\
\hline Sekretäre v. Abgeordneten & 51 & $\mathbf{0}$ & 1 & 4 & 17 & 1 & $\mathbf{0}$ & 2 & 76 \\
\hline Parteifunktionäre/Mitarbeiter & 2 & 3 & 12 & 4 & 15 & 0 & 0 & 0 & 36 \\
\hline Gewerkschaften & $\mathbf{0}$ & 4 & $\mathbf{0}$ & 7 & 8 & $\mathbf{0}$ & $\mathbf{0}$ & $\mathbf{0}$ & 19 \\
\hline Universitätsdozenten & 3 & 2 & 1 & 2 & 5 & 0 & 0 & 0 & 13 \\
\hline Medienberufe & 9 & 0 & 0 & 4 & 2 & 0 & 0 & 0 & 15 \\
\hline Anwälte & 6 & 0 & 4 & 8 & 11 & 0 & 0 & 0 & 29 \\
\hline Lehrer & 0 & 0 & 0 & 1 & 0 & 0 & 0 & 1 & 2 \\
\hline Ärzte, Zahnärzte & 2 & 0 & 1 & 1 & 4 & 0 & 0 & 0 & 8 \\
\hline $\begin{array}{l}\text { Angestellte öffentlicher Un- } \\
\text { ternehmen }\end{array}$ & 14 & 0 & 0 & 2 & 6 & 0 & 0 & 1 & 23 \\
\hline $\begin{array}{l}\text { Bürgerrechtsbewegungen, } \\
\text { Verbraucherschutz }\end{array}$ & 0 & 3 & 0 & 1 & 0 & 0 & 0 & 0 & 4 \\
\hline Unternehmer & 13 & 0 & 0 & 1 & 3 & 0 & 0 & 1 & 18 \\
\hline Freie Berufe, Selbständige & 0 & 0 & 0 & 1 & 1 & 0 & 0 & 0 & 2 \\
\hline $\begin{array}{l}\text { Landwirtschaftliche Genos- } \\
\text { senschaften }\end{array}$ & 1 & 0 & 0 & 1 & 0 & 0 & 0 & 0 & 2 \\
\hline Religiöse Vereinigungen & 0 & 0 & 0 & 0 & 3 & 0 & 1 & 0 & 4 \\
\hline $\begin{array}{l}\text { Angestellte sonstiger Verbän- } \\
\text { de }\end{array}$ & 1 & 0 & 0 & 0 & 8 & 0 & 0 & 0 & 9 \\
\hline Andere & 6 & 1 & 0 & 2 & 8 & 0 & 0 & 0 & 17 \\
\hline Sitze im Parlament & 239 & 15 & 26 & 52 & 156 & 2 & 1 & 9 & 500 \\
\hline
\end{tabular}

Nach: Asahi Shinbun: Senkyô-Taikan 1996, Asahi Shinbun-sha, Tôkyô 1996, S. 116f. (Asahi Shinbun: Ein Überlick über die Wahl 1996).

\section{Abbildung 27: Japanische Unterhausabgeordnete: Bildungsstrukturen}

\begin{tabular}{l|c|c|c|c} 
& $\mathbf{1 9 7 7}$ & $\mathbf{1 9 8 7}$ & $\mathbf{1 9 9 3}$ & $\mathbf{1 9 9 6}$ \\
\hline Tôkyô Daigaku & 116 & 103 & 96 & 95 \\
\hline Waseda & 61 & 61 & 66 & 73 \\
\hline Keiô & 28 & 42 & 53 & 46 \\
\hline Chûô Daigaku & 32 & 38 & 42 & 32 \\
\hline Kyôto Daigaku & 20 & 16 & 18 & 21 \\
\hline Gesamt & $\mathbf{2 5 7}$ & $\mathbf{1 8 8}$ & $\mathbf{2 7 5}$ & $\mathbf{2 6 7}$
\end{tabular}

Nach: Tanaka, Aiji: Nihon, in: Yoshino, Takashi; Imamura, Osamu; Tanitô, Etsushi (Hrsg.): Dare-ga Seijika-ni naru-no-ka - Kôhoshaerabi-no Kokusaihikaku, Waseda-Daigaku Shuppanbu, Tôkyô 2001, S. 21. (Tanaka, Aiji: Japan, in: Yoshino, Takashi et.al.: Wer wird Politiker? Ein internationaler Vergleich der Kandidatenauswahl.)

\footnotetext{
${ }^{494}$ An dieser Stelle wird von der Asahi Shinbun nicht nach Beamten auf lokaler Ebene und nationaler Ebene unterschieden, sondern nach Dienststellen und höheren bzw. mittlerem Dienst. Interessant ist hierbei, dass größte Gruppe, 20 Personen, von der mittleren Führungsebene des Finanzministeriums gestellt wird.
} 


\subsection{Leitfadeninterviews mit Erbabgeordneten}

\subsubsection{Interviewleitfaden 2003}

\section{Fragekomplex}

- Schlüsselfragen

o Eventualfragen

\section{Einstiegsfragen}

- Wie sind Sie in die Politik gekommen?

どうして政治の道に進んだのか。

o Haben sie den Wahlkreis ihres Vaters etc. übernommen? 前任者から選挙区を受け継いだのか。

o Welche Anreize bot der Einstieg in die Politik? どのようなモチベーションをもって政治家になったのか。

o Gab es Druck seitens der Familie in die Politik zu gehen? Oder gab es Ablehnung?

政治家になる上で家族の側からの圧力があったか。または家族の反対が あったのか。

\section{Erbabgeordnete unter dem 1955er-System}

- Wie erklären Sie die hohe Zahl von Erbabgeordneten unter dem 1955er-System?

55 年体制において世襲代議士が多数を占めた原因をどのように考えるのか。

o Welche Rolle spielte das Wahlsystem?

選挙制度が影響をもたらしたのか。

o Welche Rolle spielte die Politikfinanzierung?

政治資金が影響をもたらしたのか。

o Welche Rolle spielte die Parteienstruktur?

政党内の構造が影響をもたらしたのか。

- Welche Anreize, welche Zwänge gab es für Erbabgeordnete in die Politik zu gehen? 
一般的にどのようなモチベーションから世襲代議士が生まれたのか。

- Wie wurde unter dem 1955er-System dieses Phänomen bewertet?

55 年体制にはこの現象がどのように捉えられていたのか。

\section{Die Reformen nach 1994}

- Welche Folgen hatten die Reformen für Sie und Ihren Wahlkreis?

あなたとその選挙区に政治改革はどのような結果をもたらしたのか。

o Hat sich der Einfluss der Partei verändert?

政党が及(およ)ぼす影響に変化があったか。

o Hat sich die Bedeutung der kôenkai verändert?

後援会が及(およ)ぼす影響に变化があったか。

- Haben sich die Rekrutierungsmechanismen verändert?

候補者の選択の仕組みに変化があったか。

o Wer bestimmt jetzt die neuen Kandidaten?

小選挙区の候補者は誰がどのように決定するのか。

\section{Chancen für die Weitergabe eines Mandats}

- Haben Sie bereits einen Nachfolge bestimmt?

すでに後継者を決めているのか。

Falls Antwort „Nein“:

- Wer wird dann den Wahlkreis übernehmen?

それでは誰が受け継ぐことになると考えるのか。

- Wer wird den Nachfolger bestimmen?

誰が決めることになるのか。

- Warum übernimmt kein Verwandter etc. den Wahlkreis?

なぜ親戚の誰が゙受け継がないのか。

o Hätten Sie gern den Wahlkreis einem Verwandten übergeben?

親戚に受け継いでほしかったか。 
o Gibt es keine Motivation seitens eines Familienmitgliedes zur Übernahme des Wahlkreises?

親戚の誰かにはモチベーションがないのか。

Falls Antwort „Ja“:

- Warum haben Sie ihn [den Nachfolger, z.B. den Sohn] ausgewählt?

どうして彼に決めたのか。

- Warum übernimmt er [der Nachfolger, z.B. der Sohn] den Wahlkreis?

どうして彼が選挙区を受け継ぐのか。

o Über welche Motivationen verfügt er?

彼がどのようなモチベーションを持っているのか。

o Über welche Fähigkeiten verfügt er? Verfügt er beispielsweise über Erfahrungen in der Regional- oder Lokalpolitik?

彼は政治家としてどのような能力を持っているのか。例えば、県議員、 地方議員としての経験を持っているのか。

o Wie ist die Meinung in der kôenkai zu dem Nachfolger? その後継者について後援会はどのように考えるのか。

o Wie ist die Meinung in der Partei zu dem Nachfolger? その後継者について政党はどのように考えるのか。

- Welche Chancen rechnen sie ihrem Nachfolger aus?

その後継者が当選するチャンスはどの程度あると考えるのか。

\section{Zukunft der Erbfolge}

- Gibt es eine Zukunft für Erbfolge in den Wahlkreisen unter den neuen Bedingungen des politischen Systems?

新しい選挙制度において世襲代議士の将来はどのようになるのか。

o Wird die Zahl der Erbabgeordneten Zu- oder Abnehmen?

世襲代議士の数が増加・減少するのか。 
o Gab es nicht auch im Oberhaus unter ähnlichen Bedingungen Erbabgeordnete?

しかし、参議院にも同じ選挙制度で世襲代議士がいた。

o Gibt es dabei Unterschiede zwischen Einerwahlkreisen und Listenwahlsystem? 比例代表制と小選挙区制で違いがあるのか。

o Bewerten Sie Erbabgeordnete als überwiegend positiv oder negativ?

一般的には世襲代議士という現象についてどのように考えているか。ポ ジティブまたはネガティブに考えるのか。

- Welche Veränderungen wird es Ihrer Meinung nach bei der Kandidatenrekrutierung geben?

これから候補者選択の仕組みにどのような変化があると思うか。 


\subsubsection{Interviewleitfaden 2005}

\section{Fragekomplex}

- Schlüsselfragen

o Eventualfragen

\section{Einstiegsfragen}

- Wie sind Sie in die Politik gekommen?

どうして政治の道に進んだのか。

o Haben sie den Wahlkreis von einem Verwandten übernommen?

親戚から選挙区を受け継いだのか。

o Welche Motivation gab es, in die Politik zu gehen?

どのようなモチベーションをもって政治家になったのか。

o Gab es Druck seitens der Familie in die Politik zu gehen? Oder gab es Ablehnung? Welchen Einfluss hatte das Aufwachsen in einer Politikerfamilie?

政治家になる上で家族の側からの圧力があったか。または家族の反対が あったのか。政治家の家で育ったことでどのような影響があったか。

o Welche politischen Erfahrungen hatten Sie?

それ以前に政治的活動の経験があったのか。

- Welche Rolle spielten die Erwartungen der kôenkai?

後援会側からの期待または反対があったか。

\section{Erbabgeordnete unter dem 1955er-System}

- Wie erklären Sie die hohe Zahl von Erbabgeordneten unter dem 1955er-System?

55 年体制において世襲代議士が多数を占めた原因をどのように考えるのか。

o Welche Rolle spielten das Wahlsystem und die Politikfinanzierung?

選挙制度、または政治資金などが影響をもたらしたのか。

- Welche Anreize, welche Zwänge gab es für Erbabgeordnete in die Politik zu gehen?

一般的にどのようなモチベーションから世襲代議士が生まれたのか。 
- Wie wurde unter dem 1955er-System dieses Phänomen bewertet?

55 年体制にはこの現象がどのように捉えられていたのか。

\section{Auswirkungen der politischen Reformen auf den eigenen Wahlkreis.}

- Welche Folgen hatten die Reformen für Sie und Ihren Wahlkreis?

あなたとその選挙区に政治改革はどのような結果をもたらしたのか。

o Hat sich die Bedeutung der kôenkai verändert?

後援会が及ぼす影響に変化があったか。

o Welche Rolle spielt die Partei bei der Auswahl der Kandidaten?

政党が及ぼす影響に変化があったか。

- Haben Sie bereits einen Nachfolge bestimmt?

すでに後継者を決めているのか。

Falls Antwort „Nein“:

- Wer wird dann den Wahlkreis übernehmen?

それでは誰が受け継ぐことになると考えるのか。

- Wer wird den Nachfolger bestimmen?

誰が決めることになるのか。

- Warum übernimmt kein Verwandter etc. den Wahlkreis?

なぜ親戚の誰かが受け継がないのか。

o Hätten Sie gern den Wahlkreis einem Verwandten übergeben?

親戚に受け継いでほしかったか。

Falls Antwort „Ja“:

- Warum haben Sie ihn [den Nachfolger, z.B. den Sohn] ausgewählt? どうして彼に決めたのか。

- Über welche Motivationen verfügt er?

彼がどのようなモチベーションを持っているのか。 
- Über welche Fähigkeiten und Erfahrungen (z.B. in der Lokalpolitik) verfügt er? 彼は政治家としてどのような能力を持っているのか。例えば、県議員、地 方議員としての経験を持っているのか。

o Wie ist die Meinung in der kôenkai zu dem Nachfolger? その後継者について後援会はどのように考えるのか。

o Wie ist die Meinung in der Partei zu dem Nachfolger? その後継者について政党はどのように考えるのか。

\section{Die allgemeinen Auswirkungen der politischen Reformen auf die Erbfolge}

- Wie haben sich die Rekrutierungsmechanismen insgesamt verändert? 候補者の選択の仕組みに変化があったか。

o Wer bestimmt jetzt die neuen Kandidaten? 小選挙区の候補者は誰がどのように決定するのか。

o Welchen Einfluss haben die kôenkai auf die Auswahl der Kandidaten? Welchen Einfluss haben die offiziellen Parteiorganisationen?

後援会が候補者選びに及ぼす影響に変化があったか。 政党が候補者選びに及ぼす影響に変化があったか。

o Welche Anreize bestehen noch für Erbabgeordnete? 現在の政界において世襲代議士に有利な条件があるとすれば何か。

o Haben Erbabgeordnete noch Vorteile gegenüber ihren Konkurrenten? 選挙の際、同一選挙区の他の候補者に比べ世襲代議士の方が有利である か。それで何故か。

- Gibt es eine Zukunft für Erbfolge in den Wahlkreisen unter den neuen Bedingungen des politischen Systems?

新しい選挙制度において世襲代議士の将来はどのようになるのか。

o Wird die Zahl der Erbabgeordneten Zu- oder Abnehmen? 世襲代議士の数が増加・減少するのか。

o Gibt es dabei Unterschiede zwischen Einerwahlkreisen und Listenwahlsystem? 
比例代表制と小選挙区制で違いがあるのか。

- Welche Veränderungen wird es Ihrer Meinung nach bei der Kandidatenrekrutierung geben?

これから候補者選択の仕組みにどのような変化があると思うか。

- Gibt es Probleme, geeignete Nachwuchskräfte zu finden?

適当な後継者探しに困難を感じているか。

- Bewerten Sie Erbabgeordnete als überwiegend positiv oder negativ?

一般的には世襲代議士という現象についてどのように考えているか。ポジティ ブまたはネガティブに考えるのか。 


\subsubsection{Interviewpartner}

Mit folgenden Politikern wurden im März/April 2003 im Rahmen eines DAADKurzzeitstipendiums für Abschlussarbeiten Interviews geführt:

o Ôno Yoshinori, LDP, geboren 1941, Wahlkreis Kagawa 3, Abgeordneter in zweiter Generation. Ônos Schwiegervater war Unterhausabgeordneter, sein früh verstorbener Vater Gouverneur der Präfektur Kagawa. Ôno Yoshinori war zunächst im Außenministerium tätig und trat 1978 bei den Gouverneurswahlen von Kagawa an, die er allerdings verlor. 1986 wurde Ôno ins Unterhaus gewählt. Ôno Yoshinori war unter anderem stellvertretender Bildungsminister und Chef des Verteidigungsamtes (Verteidigungsminister).

o Usui Hideo, LDP, geboren 1939, Wahlkreis Chiba 1, Abgeordneter in zweiter Generation. Usui Hideo war als Sekretär seines Vaters tätig und übernahm nach dessen Rücktritt den Wahlkreis. Er war in verschiedenen Kabinetten als Minister tätig, u.a. als Justizminister und Chef des Verteidigungsamtes (Verteidigungsminister). Sein Sohn, Usui Shôichi, wurde im April 2003 ins Parlament der Präfektur Chiba gewählt. Zum Zeitpunkt der zweiten Befragung war Usui Hideo LDP-Parteivorsitzender von Chiba und nicht als Abgeordneter tätig. Seit 2005 sitzt der Politiker aber wieder im japanischen Unterhaus.

o Itô Shintarô, LDP, geboren 1951, Wahlkreis Miyagi 4, Abgeordneter in zweiter Generation. Itô war als Nachrichtensprecher, Regisseur und Universitätsdozent tätig, bevor er Sekretär seines Vaters Itô Soichirô wurde, dem ehemaligen Sprecher des Unterhauses. Nach dessen Tod übernahm er den Wahlkreis Miyagi 4.

o Hamada Yasukazu, LDP, geboren 1955, Wahlkreis Chiba 12, Abgeordneter in zweiter Generation. Hamada ist ein interessanter Fall, da er sich in seinem Wahlkreis mit einem anderen Abgeordneten, Nakamura Shôzaburô, nach dem so genannten „Costa-RicaSystem“ abwechselte. Hamada war vor seiner Abgeordnetentätigkeit Sekretär seines Vaters.

o Matsuno Yorihisa, DPJ, geboren 1960, Wahlkreis Kumamoto 1, Abgeordneter in dritter Generation. Matsuno war in der Parteizentrale der Nihon Shintô und später in der DPJ in verschiedenen Positionen tätig. Matsuno wurde Sekretär des ehemaligen Ministerpräsidenten und Begründers der Nihon Shintô, Hosokawa Morihiro, dessen Wahlkreis er übernahm.

o Watanabe Shû, DPJ, geboren 1961, Shizuoka 6, Abgeordneter in zweiter Generation. Vor seiner Tätigkeit als Unterhausabgeordneter war Watanabe Redakteur bei der „Yomiuri Shinbun“-Zeitung und Abgeordneter des Regionalparlaments von Shizuoka. Der jüngere Bruder von Watanabe Shû, Watanabe Jin, ist sein Sekretär. 
Mit folgenden Politikern wurden von Februar bis Mai 2005 im Rahmen eines DAADKurzzeitstipendiums für Doktoranden Interviews geführt:

o Harada Yoshitsugu, LDP, geboren 1952, Wahlkreis Shizuoka 2, Abgeordneter in zweiter Generation. Harada war als Journalist und Leiter der Presseabteilung bei NHK tätig. Als sein Vater Harada Shôzô schwer erkrankte, übernahm er dessen Wahlkreis.

o Hori Kôsuke, LDP, geboren 1934, Wahlkreis Saga 3, Abgeordneter in zweiter Generation. Hori war für eine japanische Firma in Frankreich tätig, als er den Wahlkreis seines verstorbenen Vaters übernahm. Der Abgeordnete war u.a. als Bildungsminister tätig.

o Funada Hajime, LDP, geboren 1953, Wahlkreis Tochigi 1, Abgeordneter in zweiter Generation. Funada übernahm nach dem Tod seines Großvaters dessen Wahlkreis. Sein Vater war Gouverneur von Tochigi. Funada Hajime galt als „Prinz“ der LDP und war mit 39 Jahren jüngster Minister der japanischen Nachkriegszeit. In den 1990er Jahren gehörte Funada der Shinshintô an. Nach einem Skandal verlor er zwischenzeitlich sein Mandat.

o Ishiba Shigeru, LDP, geboren 1957, Wahlkreis Tottori 1, Abgeordneter in zweiter Generation. Ishibas Vater war Oberhausabgeordneter, weshalb es keine Unterstützerorganisation gab, die er übernehmen konnte. Zu Beginn der 1990er Jahre setzte sich Ishiba für politische Reformen ein und wechselte in die Shinseitô, die er nach zwei Jahren wieder verließ. Ishiba trat 1998 als unabhängiger Kandidat an und kehrte in die LDP zurück.

o Kômura Masahiko: geboren 1942, Wahlkreis Yamaguchi 1, Abgeordneter in zweiter Generation. Kômura, gelernter Rechtsanwalt, übernahm den Wahlkreis seines Vaters und ist heute Faktionsführer der gleichnamigen habatsu.

o Kosaka Kenji, LDP, geboren 1946, Wahlkreis Nagano 1, gehört zu den wenigen Abgeordneten, deren Familie bereits in 4. Generation in der Politiktätig ist. Kosaka Kenji arbeitete für Japan Airlines in England, bevor er seine politische Karriere als Abgeordneter des Präfekturparlaments von Nagano begann.

Abweichende Fälle:

o Terata Manabu, DPJ, geboren 1976, Wahlkreis Akita 1. Terata stellt nicht nur wegen seines Alters eine Ausnahme dar, sein Vater war als Gouverneur von Akita nur auf regionaler Ebene tätig. Deshalb ist Terata kein Erbabgeordneter im engeren Sinn, stellt aber einen interessanten Fall dar, da er sich intensiv mit politischer Rekrutierung beschäftigt.

o Usui Shôichi, LDP, geboren 1975, Wahlkreis Mihama-ku. Der Sohn von Usui Hideo ist ein abweichender Fall, da er als Präfekturabgeordneter von Chiba nur auf regionaler Ebene tätig ist. Dennoch ist er ein interessanten Fall, da sich hier ein Erbfolge beobachten läßt. Vor seiner politischen Karriere war Usui Shôichi bei Disney-Land Tôkyô beschäftigt. 


\subsubsection{Beispielinterview mit Ôno Yoshinori am 21.4.2003}

- Herr Ôno, wie sind Sie in die Politik gekommen?

Ich habe während meiner Studienzeit an einem Studentenaustausch mit den USA teilgenommen. Das war von 1961 bis 1963. Man träumte damals davon, nach Amerika gehen zu können. Auch wenn die Eltern reich waren, war es sehr schwer, in den USA studieren zu können. Aber weil ich einen Test bestanden hatte, bekam ich ein Fulbright-Stipendium.

Damals gab es solche Dinge wie Highways oder fließendes heißes Wasser noch nicht in Japan. Die Straßen war schlecht. Ich sah die Zentralheizungen in Amerika und dachte: „Das wäre was, wenn es die in Japan geben würde.“ Richtige Supermärkte hatten wir auch noch keine in Japan. Es gab also einen großen Unterschied in der Lebensqualität. Ich wollte den Lebensstandard Japans dem Amerikas angleichen. Das war der Hintergrund.

Der direkte Einstieg war 1978. 1978 waren die Gouverneurswahlen in Kagawa. Der damalige Gouverneur war von der SPJ. Ôhira Masayoshi war in dieser Zeit Kandidat für den LDP-Vorsitz. Er dachte daher, man müsse Kagawa-ken für die LDP gewinnen. „Wer ist wohl dafür geeignet“, dachte er. So kam man auf mich. Ich wollte nämlich in die nationale Politik. Ministerpräsident Miki Takeo hatte mir gesagt: „Die Arbeit des Gouverneurs ist, abgesehen von Außenpolitik und Außenhandel, die Arbeit, die am meisten der des Ministerpräsidenten ähnelt.“ Das war natürlich eine Herausforderung.

So kam es zum Kampf zwischen der LDP und der SPJ. Mein Gegner war also von der SPJ, ich war neuer Kandidat der LDP. Im Wahlkampf sagt man sehr viele schlechte Dinge über einander. Das größte Problem aber dabei war, dass der Abgeordnete Katô Tsunetarô mein Schwiegervater war. Er hatte eine Fährgesellschaft. Zu dieser Zeit sollte aber die Seto-Ô-Hashi-Brücke gebaut werden. Um nun diese Fährgesellschaft zu retten, sollte ich Gouverneur werden. So sagte man jedenfalls.

Dieses Gerücht hielt sich hartnäckig, obwohl es nicht so war. Ich habe es damals so oft gesagt. Ôhira hatte mich ja als Kandidaten ausgesucht. Aber dennoch verlor ich knapp. Danach war ich 8 Jahre Rônin. Kennen Sie den Begriff? „Unemployed warrior“ auf Englisch. Dann stellte ich mich wieder zur Wahl.

o Gab es einen Einfluss seitens der Familie?

Als mein Vater starb, war ich 28. Daher gab es diesen Einfluss nicht. Ich fühle mich also nicht wie ein Erbabgeordneter.

o Haben Sie den Wahlkreis Ihres Vaters übernommen?

Ich habe die kôenkai meines Vaters übernommen, aber sie war vergleichsweise klein.

- Wie erklären Sie sich die hohe Zahl von Erbabgeordneten unter dem 1955er-System?

Damals gab es die Mehrerwahlkreise. Ob damals die Zahl der Erbabgeordneten stark anstieg, kann ich nicht sagen. Damit habe ich mich nicht befasst. Damals wurde die LDP ewige Regierungspartei, nicht wahr? Stieg damals die Zahl der Erbabgeordneten so plötzlich an?

o Der große Anstieg war in den 1960er Jahren.

Es gab einen Wechsel der Abgeordneten, nicht wahr? Die Abgeordneten, die um 1955 gewählt wurden, stellten einen Generationswechsel dar, nicht? Es gab nicht so viele Abgeordnete, die seit der Vorkriegs- 
zeit in der Politik waren. Das politische System der Nachkriegszeit war ganz anders. Als es den Wechsel der Abgeordneten gab, stieg die Zahl, oder?

o $\quad$ Also das Phänomen der Erbabgeordneten ist ein Phänomen der Nachkriegszeit?

Ja, das ist ein Phänomen der Nachkriegszeit. Kurz gesagt gab es vorher ein System mit Einerwahlkreisen und zwei Parteien. Als die Mehrerwahlkreise eingeführt wurde, gab es ein Parteiensystem mit vielen Parteien. Das Wahlsystem spielte die größte Rolle unter den verschiedenen Gründen, denke ich.

Das heißt, die Mehrerwahlkreise waren der wichtigste Grund für die große Zahl der Erbpolitiker... Es ist nicht von der Hand zu weisen, dass die Mehrerwahlkreise einer der wichtigsten Gründe waren. Die große Zahl der Erbpolitiker war außerdem mit der Regierungspartei verbunden. Warum...?!

Erstens war es einfacher für Kandidaten, unter diesem Wahlsystem Mandate zu bekommen. Zum Beispiel bekamen in einem Wahlkreis drei Kandidaten von der gleichen Partei ein Mandat. Wenn nun ein Kandidat starb, war es einfach für den Sohn, aus Mitleid gewählt zu werden. Das stand in keiner Beziehung zu den tatsächlichen Fähigkeiten des Kandidaten. „Der arme Sohn“, hieß es dann, „den müssen wir unterstützen.“

Der zweite Grund waren die kôenkai. Die Kandidaten wurden nicht aufgrund der Stärke einer Partei gewählt, sondern aufgrund der kôenkai, die sie aufgebaut hatten. In England beispielsweise zählt die Stärke der Partei. Daher gibt es dort kaum Erbpolitiker. In Deutschland ist es das gleiche, oder?

In Japan zählt die Stärke der kôenkai, das ist ein Charakteristikum des 1955er-System, das bis heute in den Einerwahlkreisen fortbesteht. Warum werden Erbpolitiker gewählt? Es ist wegen der Stärke ihrer kôenkai. Eine kôenkai aufzubauen ist sehr kostenintensiv. Die Versammlungen, Essen und Trinken kosten viel Geld. Obwohl die Wahlgesetze da eigentlich sehr streng sind...

In den kôenkai sind Leute, die einen persönlichen Gewinn erwarten. Wenn man diese kôenkai aufgebaut hat, denken die Mitglieder: „Wie rührend!“. Zum zweiten bedanken sich die Kandidaten für die Unterstützung, indem sie beispielsweise Arbeitsplätze verteilen. Wenn man also viel Geld eingesetzt hat, um die kôenkai aufzubauen, denkt man, dass es schade wäre, sie nicht an einen Nachfolger weiterzugeben. Daher wird dann der Sohn zum Nachfolger. Deshalb ist die Zahl der Erbpolitiker angestiegen. Der Hauptgrund ist also das Wahlsystem.

Wie wird sich das also unter dem neuen Wahlsystem entwickeln? Da befinden wir uns noch in der Testphase. Unter dem neuen System gab es zwei Wahlen, aber nach wie vor gibt es Dritt- und Viertgenerationspolitiker, oder? Immer noch sind die kôenkai die Basis der Kandidaten.

Zum Beispiel: Der Wahlkreis Kagawa 3 ist der Wahlkreis, aus dem auch Ôhira Masayoshi kam. Sein Schwiegersohn ist Herr Morita. Ôhira konnte normalerweise 60.000 bis 70.000 Stimmen gewinnen. Als Ôhira starb, konnte der Schwiegersohn auf Anhieb mehr als 15.000 Stimmen gewinnen. Das waren Sympathiestimmen oder Stimmen von Leuten, die durch ihre Interessen mit Ôhira verbunden waren. In unserem Mehrerwahlkreis waren wir drei Abgeordnete. Als der Wahlkreis zum Einerwahlkreis wurde, gewann ich ihn. Die anderen beiden kôenkai sind aber noch da. Sie sind überhaupt nicht zusammengewachsen. Das ist typisch japanisch. Wenn wir ein System hätten, in dem Parteien stärker wären, gäbe es keine Erbabgeordneten mehr.

Das heißt, es ist fraglich, ob es gut ist, dass die Wahlen weiterhin durch solche zwischenmenschlichen Beziehungen geprägt sind. So könnte auch ein Kandidat aufgrund der Parteizugehörigkeit gewählt werden, der nicht über solche Beziehungen verfügt, weil die Partei ihn berufen hat. Der Hintergrund zählt in 
Japan bei den Erbabgeordneten. Das heißt, dass attraktive Kandidaten mit entsprechendem familiären Hintergrund eine größere Chance haben als sie nur aufgrund der Parteizugehörigkeit hätten.

Wenn man wie die LDP Regierungspartei ist, „government party“, nicht wahr, kann man, wenn man die Macht lange erhält, Geld in die kôenkai leiten. Das ist ein großer Vorteil. Die Oppositionsparteien haben diese Möglichkeiten nicht. Jetzt in den Einerwahlkreisen sind diese Probleme übrig geblieben wie etwa die Zentrierung auf zwischenmenschliche Beziehungen und kôenkai, also dass es keine Parteibindung gibt.

- $\quad$ Welche Folgen hatten die Reformen für Sie und Ihren Wahlkreis?

Was gab es da an politischen Reformen? Es gab ja eine ganze Reihe von Problemen. Aber vereinfacht gesagt, wurden aus den Mehrerwahlkreisen Einerwahlkreise. Vorher waren also 3 bis 5 Kandidaten in einem Wahlkreis gewählt worden, nicht wahr.

Eine große Motivation, die Reformen durchzuführen, war den Geldmitteleinsatz in den Wahlkämpfen zu verringern und damit auch die Korruption in der Politik zu vermindern. In Mehrerwahlkreisen traten mehrere Kandidaten der gleichen Partei gegeneinander an und brauchten dafür viel Geld. Der Kampf unter Kandidaten der gleichen Partei war sehr teuer. Es war ja kein Wahlkampf zwischen Parteien. Das war also ein Grund: Das Problem der teuren Wahlkämpfe zu lösen. Wenn Politiker viel Geld brauchen, werden sie korrupt.

Es gab noch ein anderes Problem, aber das war das größte Problem. Das andere Problem: Was bedeutete das 1955er-System? Das 1955er-System bedeutete, dass es eine ewige Regierungspartei und eine ewige Oppositionspartei gab. Es gab kein Konkurrenzverhältnis. Es gab immer nur eine regierende Partei. Daher wollte man ein System schaffen, in dem der Machtwechsel möglich ist. Das ist nicht ein System in dem Kandidaten der gleichen Partei sich mit ihrem Geld bekämpfen. Daher sagte man sich, dass ein Einerwahlkreis-System besser sei, in dem es einen Kandidaten der LDP und einen anderen Kandidaten gibt und ein Machtwechsel möglich ist.

Man strebte also ein System an, in dem der Regierungswechsel von Parteien auf Basis der Parteipolitik möglich war. Man strebte ein System an, in dem weniger Geld verbraucht wird. Das war die Basis der politischen Reformen.

Das erste, was sich also geändert hat, war, dass man ein Gesetz verabschieden musste, das die Wahlkampfkosten begrenzt. Also hat man das Gesetz über die politische Finanzierung verabschiedet. So wurde transparenter, wie viel Geld ein Kandidat im Wahlkampf ausgibt. Außerdem wurde ein System geschaffen, in dem der Machtwechsel möglich war. Das war das Wichtigste.

Wie weit sich das alles auf die Politikerfamilien auswirkt, das heißt auf die Erbabgeordneten, das ist noch in der Testphase. Dass es also soweit kommt, dass in den Wahlkreisen die Wähler sagen, ,ah, das ist der Sohn von dem und dem, das geht aber nicht“, dass man sagt: „Das System der Erbfolge ist nicht gut“, wäre ein Fortschritt. Aber das ist nicht die Norm. Das befindet sich noch in der Testphase.

- $\quad$ Haben sich die Rekrutierungsmechanismen verändert?

Das ist wirklich eine schwere Frage... 
o $\quad$ Also, dass beispielsweise der Einfluss der Partei stärker geworden ist...

Bei den Unterhausabgeordneten wird der offizielle Kandidat der Partei ja eigentlich zentral bestimmt. Aber die Leute im Wahlkreis, die sagen, „ich möchte mich aufstellen lassen, ich möchte mich aufstellen lassen“ können einfach ohne jede Voraussetzung antreten. Wenn sie die Wahl dann gewonnen haben, wechseln sie in die LDP.

Man muss diese Sache unter zwei Gesichtspunkten sehen. Für Kandidaten, deren Eltern Abgeordnete waren, also Erbpolitiker, sind die Möglichkeiten nach wie vor recht gut, gewählt zu werden. Wer nun aber als offizieller Kandidat aufgestellt wird, wird zentral entschieden. Das machen die Gremien der Präfektur, also beispielsweise die Kagawa-Kenrengôkai. Da hat sich prinzipiell nicht viel verändert.

Aber ich persönlich denke, dass es gut wäre, wenn es ein System wie in Amerika mit den Vorwahlen geben würde. Der Einfluss der Parteimitglieder in Japan ist sehr gering. Von 300.000 Einwohnern sind 30.000 Parteimitglieder.

o $\quad$ Aber die sind kaum aktiv.

Nein, nicht aktiv, die sind bloß in die Partei eingetreten. Die Parteimitglieder und meine kôenkai sind zusammengewachsen. Aber unter den anderen Parteimitgliedern sind oft welche, die einen anderen Kandidaten unterstützen. Dieses Problem müsste man öffentlich diskutieren.

Zur Zeit wird die Auswahl der Kandidaten entweder durch Erbfolge oder die Parteizentrale vorgenommen. Daher frage ich mich, ob es nicht gut wäre, wenn es Vorwahlen geben würde. Die Kandidaten würden dann miteinander in Konkurrenz treten. Dann würde es auch weniger Erbabgeordnete geben.

Die andere Sache ist die Qualität der Kandidaten. Wenn man über die Qualität der Kandidaten nachdenkt, bemerkt man, dass die LDP viele Erbabgeordnete hervorgebracht hat. Die Kandidatenplätze werden hauptsächlich von Erbabgeordneten eingenommen. Die Ausübung einer Funktion in der Partei, die Tätigkeit in der Lokalpolitik oder die Erbfolge sind die drei Möglichkeiten, die als Vorraussetzung für eine Kandidatur für die LDP gelten. Doch die Erbabgeordneten gewinnen meistens. Die Funktionsträger aus der Partei, die keine Erbabgeordneten sind, wechseln dann oft in die DPJ. Das macht mir ein wenig Angst.

- $\quad$ Haben Sie bereits einen Nachfolger bestimmt?

Nein, habe ich noch nicht bestimmt.

- $\quad$ Wer wird dann den Wahlkreis übernehmen? Wer wird den Nachfolger bestimmen?

Früher hat immer die kôenkai entschieden. Wenn die Familie den Sohn schickte, wurde er meist von der kôenkai unterstützt. Aber das muss aufhören. Meiner Meinung nach.

- $\quad$ Gibt es denn dann eine Zukunft für die Erbfolge in der japanischen Politik?

Wenn der Erbpolitiker gut ist, wird er auch Erfolg haben. Nur allein aufgrund der Erbfolge gewählt zu werden, wäre ein wenig merkwürdig, oder? Gute Nachfolger, die eine Erbfolge antreten, werden auch gute Politiker werden können. Wenn sich der Sohn aber nicht so für die Politik interessiert, sollte er einen anderen Weg gehen. Zum Beispiel sollte die Familie Ôno einmal Pause machen... Das Zeitalter, in dem der Sohn Politiker wurde, weil der Vater es war, geht langsam vorbei, nicht wahr. 
- Welche Veränderungen wird es Ihrer Meinung nach bei der Kandidatenrekrutierung geben?

Leider ist keine Veränderung absehbar. Man hat das Gefühl, dass es sich verändern muss, nicht wahr. Zum Beispiel ist für die Wähler immer noch wichtig, dass der Name Ôno leicht zu merken und leicht zu schreiben ist.

o $\quad$ Also der bekannte Name, das kanban des sanban-Systems.

[Lacht.] Es wäre merkwürdig, wenn dieses System nicht zu Ende ginge, nicht wahr. Zum Beispiel Bush, der Sohn von George Bush: In Amerika wird Bush nicht aufgrund der Tatsache gewählt, dass er der Sohn von Bush ist. Nur weil man den Namen Bush hört, wählt man ihn nicht. Es kann sein, dass der Hintergrund des Vaters als Präsident eine Rolle spielt, aber es ist nicht der Hauptgrund für die Wahl des Sohnes. In Japan kann der Sohn nur aufgrund dieses kanban gewählt werden. Es gibt noch keine Anzeichen, dass sich das ändert. Es wäre aber besser, wenn sich dieses System ändert. Wenn in Japan Wähler gefragt werden, ob sie einen Kandidaten wählen, nur weil er ihnen menschlich sympathisch erscheint, gibt es viele Ja-Antworten. Es kommt also häufig vor, dass die Parteizugehörigkeit weniger wichtig ist als die Sympathie. Daher führen in Japan die Politiker immer noch Wahlkämpfe weniger auf Grundlage der Parteipolitik als vielmehr auf der Grundlage von Sympathien und zwischenmenschlichen Beziehungen.

- Wird die Zahl der Erbabgeordneten zu- oder abnehmen?

Die Zahl wird abnehmen. Aber es wird nicht so sein, dass die Erbabgeordneten als Phänomen ganz verschwinden. Wahlen in Japan sind immer noch sehr personenzentriert. Daher wird das Phänomen der Erbabgeordneten weiterhin Bestand haben. 


\subsubsection{Beispielinterview mit Funada Hajime am 04.03.2005}

- Herr Funada, Wie sind Sie in die Politik gekommen?

Ja, das ist schwierig zu beantworten. Der Punkt, also der erste Punkt, ist, dass ich ja auch Erbabgeordneter bin. Mein Grossvater war 41 Jahre lang Unterhausabgeordneter. Dann starb er. Daraufhin bat die kôenkai im Wahlkreis, die politische Unterstützerorganisation, darum, dass sein Sohn oder sein Enkel die Nachfolge antreten.

Sein Sohn, also mein Vater, war zu dieser Zeit in Tochigi-ken - das ist in der Nähe von Tokyo, ganz im Norden - Gouverneur. „Es ist Unsinn, den Posten des Gouverneurs aufzugeben, um für das Parlament zu kandidieren“, sagte er. Man übersprang eine Generation, und so war ich der Nächste in der Reihenfolge. Das ist der erste Punkt. Ich hatte allerdings die Wahl, zu sagen „ich mache das nicht“. Es gibt unter den Erbpolitikern viele Leute, die den Wahlkreis nicht übernehmen. Das heisst, dass der Sohn nicht antritt, obwohl der Vater Abgeordneter ist.

Die Politik in der japanischen Nachkriegszeit, also nach Ende des Zweiten Weltkriegs, konzentrierte sich auf die Stärkung der Wirtschaft und nicht auf die Militär- und Sicherheitspolitik oder die Außenpolitik. Anders ausgedrückt: Es wurde nur für den Wirtschaftsaufschwung gearbeitet, ohne über diese Probleme nachzudenken oder irgendetwas zu investieren. Natürlich entwickelte sich die Wirtschaft sehr gut. Aber das internationale Engagement, eine Armee zum Schutz des eigenen Landes, solche grundlegenden Sachen wurden vernachlässigt. Diese Poltitikfelder sind in Japan sehr schwach ausgeprägt, da ist Japan ein schwaches Land. Ich hatte damals das Gefühl, dass sich das ändern muss.

Deshalb kann man kein Politiker werden, in gewisser Hinsicht gilt das auch für Beamte, wenn man nicht den Wunsch hat, das Land zu verändern und eine gute Politik für das Land zu machen. Ich hatte also das Ziel, Politiker zu werden, um meine eigenen Vorstellungen umzusetzen. Natürlich bin ich Erbabgeordneter, aber ich hatte eigene Pläne, die ich verwirklichen wollte. Aus diesen beiden Gründen wollte ich Politiker werden.

o Gab es Druck seitens der Familie in die Politik zu gehen? Oder gab es Ablehnung? Welchen Einfluss hatte das Aufwachsen in einer Politikerfamilie?

Seitens meiner Familie [Pause] gab es eigentlich keinen großen Widerstand. Sie meinten alle, dass ich das tun sollte. Viele waren der Meinung, dass ich, wie soll ich sagen, das Erbe meines Großvaters antreten sollte, sein Vermächtnis. Also, es gab keine Ablehnung.

- $\quad$ Wie erklären Sie die hohe Zahl von Erbabgeordneten unter dem 1955er-System?

Tja, also mit dem 1955er-System als solchem hatte die Erbfolge ja nichts zu tun. Es war das Wahlsystem. Unter dem System der Mehrerwahlkreise, also im Fall der Unterhausabgeordneten, gab es ja pro Wahlkreis drei, vier oder auch fünf Sitze. Daher hatte man die Situation, dass eine Vielzahl von Kandidaten gegeneinander antreten konnte. Das heißt, weil in den Einerwahlkreisen von der LDP oder der DPJ nur noch ein Kandidat antritt, ist eine Erbfolge schwieriger geworden.

Als es die Mehrerwahlkreise noch gab, konnte man die eigene kôenkai, also die kôenkai, die der Vater aufgebaut hatte, dem Sohn weitergeben. Durch diese Unterstützung war es überaus einfach, die Wahl zu gewinnen. Wegen dieses Umfelds (gab es so viele Erbabgeordnete - d.A.), meiner Meinung nach. 
- Wie wurde unter dem 1955er-System dieses Phänomen bewertet? Gab es Kritik in den Medien oder seitens der Wähler?

An der Erbfolge?

o $\underline{\text { Ja. }}$

Nun, ich selbst war damals erst 25 Jahre alt. Obwohl ich noch im Graduiertenseminar war, und daher noch über sehr wenig gesellschaftliche Erfahrungen verfügte, meine Karriere war ja noch nicht sehr weit gekommen, trat ich sozusagen als Jugendlicher bei einer Wahl an. Es gab Kritik, dass so jemand vom Staat Diäten bekommt. Deshalb gab es seitens der Medien einige Angriffe.

- Welche Folgen hatten die Reformen für Sie und Ihren Wahlkreis?

Es hat ja eine ganze Reihe von politischen Reformen gegeben. Ich denke, eine große Reform war die Einführung der Einerwahlkreise. So gesehen wurden zunächst einmal die Wahlkreise kleiner, nicht wahr? Zur Zeit des Mehrerwahlkreise, nun, da war es auch gut, wenn man als einer von fünf gewählten Kandidaten ins Parlament einzog. Selbst als Fünftplatzierter war es in Ordnung. Jetzt unter dem System der Einerwahlkreise muss man den ersten Platz erringen. Das heißt, dass man in den Mehrerwahlkreisen, wenn man nicht zu faul war, immer seinen Sitz behalten konnte. Seitdem es Einerwahlkreise gibt, ist es dagegen viel schwieriger geworden, gewählt zu werden.

Wenn man... [Pause], also in meinem Fall gab es ja einen kleinen Skandal, und ich wurde einmal abgewählt, nicht wahr. [Eine Affäre mit einer Oberhausabgeordneten, die er später heiratete - d.A.] Wenn man also nachlässig wird, der Ruf der LDP ist ja auch etwas schlechter geworden, dann wird man unter dem Einerwahlsystem wahrscheinlich eher verlieren. Es hat also auch die Veränderung gegeben, dass die Wahlen spannender geworden sind.

- $\quad$ Hat sich die Bedeutung der kôenkai verändert?

In den Mehrerwahlkreisen reichte es, wenn man von den Wählern ein Viertel, naja, sagen wir ein Fünftel zusammen bekam. Wenn allein die mich wählten, konnte ich gewinnen. Wenn mich jetzt unter dem Einerwahlsystem nicht die Hälfte, ja, wenn möglich mehr als die Hälfte der Wähler, unterstützt, kann ich nicht gewinnen. Kurz gesagt, reicht meine kôenkai, meine bisherige Organisation, allein nicht mehr aus. Man braucht viel mehr...

Also damals, als ich in einem Mehrerwahlkreis antrat, kamen drei Abgeordnete von der LDP. In meinem Wahlkreis wurden ja fünf Abgeordnete gewählt. [Pause] Drei Abgeordnete [von der LDP - d.A.] traten an. Und jeder hatte seine eigene kôenkai. Ich habe nun die Mitglieder der anderen beiden kôenkai gefragt, ob sie nicht in meine Organisation eintreten wollen und sie gebeten, mich zu unterstützen. Und nach und nach traten sie bei mir ein. Das heißt, die Unterstützerorganisationen wurden ausgeweitet, also die Zahl der Mitglieder.

Nun ist es so, dass es Leute gibt, die man mag, und welche, die man nicht mag. [Lacht.] Leute, die vorher für einen anderen Abgeordneten von der LDP gearbeitet haben, arbeiten jetzt für mich. Das heißt also - man kann es sich natürlich denken - dass es Leute gibt, die in Wirklichkeit das Gefühl haben: „Na ja, den Kandidaten möchte ich ja eigentlich doch nicht unterstützen." Von denen gibt es nicht viele, nur einige wenige. Aber dass solche Leute unter meinen Anhängern sind, ist doch ein großes Problem. 
- Hat sich die Rolle der Partei bei der Auswahl der Kandidaten verändert?

Ja, hat sie. In den Einerwahlkreisen tritt nur noch ein Kandidat von der LDP, ein Kandidat von der DPJ usw. an. Damit ist der Einfluss der Partei größer geworden. Wer als Kandidat antritt, entscheidet die Parteizentrale. Dann wird aus der Parteizentrale Geld, also ein Budget, für den Wahlkampf und die Aktivitäten, zur Verfügung gestellt. Dass heißt, dass man nicht Sachen sagen darf, die von der Meinung der Parteizentrale abweichen. Solche Anordnungen gibt es. Die Macht der Partei, also der Parteizentrale, ist also größer geworden. Sowohl bei der LDP als auch bei der DPJ.

o Also mehr als die Macht der lokalen Parteiorganisationen hat die Macht der Parteizentralen zugenommen?

Genau. Die Parteizentrale ist stärker.

- Haben Sie bereits einen Nachfolge bestimmt?

Meinen Nachfolger? Nein, den habe ich noch nicht bestimmt. Ich möchte noch eine Zeit lang als Abgeordneter tätig sein. [Lacht.]

- Wer wird dann den Wahlkreis übernehmen?

Ja, wenn man das unter dem Aspekt der Erbfolge sieht... also ich habe ja einen Sohn. Aber er hat kein Interesse, ihn zu übernehmen. Wenn er sagen würde, dass er das unbedingt machen möchte, würde ich ihn der kôenkai vorstellen. Danach würde ich das Urteil der kôenkai abwarten, aber von meiner Seite würde kein Druck ausgeübt werden. Ich werde nichts erzwingen. Das heißt, ich habe auch nicht die Absicht, von der Parteizentrale zu fordern, dass mein Sohn auf jeden Fall aufgestellt wird.

- Wir haben ja gerade über die Auswirkungen der Reformen auf Ihren Wahlkreis gesprochen. Wie haben sich die politischen Reformen denn allgemein auf die Rekrutierungsmechanismen ausgewirkt?

Die Rekrutierungsmechanismen?

o $\mathrm{Ja}$, in der LDP.

Innerhalb der LDP? [Pause] Die Mechanismen der Kandidatenauswahl...

o Also zum Beispiel die Einführung von Vorwahlen.

Ach so. Ja, in einem Wahlkreis, in dem es keinen Nachfolger mehr gibt, also in einem Wahlkreis ohne LDP-Kandidaten, gibt es einen Aufruf. Nun kann es vorkommen, dass es viele Leute gibt, die kandidieren möchten. Gibt es zum Beispiel zwei oder drei mögliche Kandidaten, wird unter den Parteimitgliedern eine Vorwahl durchgeführt. Wenn also eine Vorwahl durchgeführt wird und die LDP-Mitglieder entscheiden, dass sie diesen Kandidaten wünschen, wird die Zentrale diesen Kandidaten anerkennen und aufstellen. Dieses System wurde eingeführt.

Wenn es einen Kandidat gibt, der bereits ein- oder zweimal als Kandidat der LDP gewonnen hat, wird bei der nächsten Wahl der derzeitige Amtsinhaber automatisch Kandidat. Es sei denn, er hat in seinem Wahlkreis äußerst schlechte Sachen gemacht. Beispielsweise, wenn er festgenommen oder verhaftet wurde, nicht wahr. [Lacht.] Oder wenn seine körperliche Verfassung so schlecht ist, dass er aufhören muss. An dieser Situation hat sich nichts verändert. 
o Wie hat sich der Einfluss der kôenkai verändert?

Der Einfluss der kôenkai bei der Kandidatenauswahl? Deren Einfluss ist dagegen schwächer geworden, nicht wahr. Schwächer, würde ich sagen. Nehmen wir einmal an, ich würde bei der nächsten Wahl verlieren, aber sagen „ich versuche es noch einmal“ und die kôenkai würde auch so denken. Die Parteizentrale könnte dann sagen: „Wahrscheinlich ist Dein Gegner doch stärker als Du, wenn Du kandidierst. Deshalb tritt jemand anders an." Solche Fälle gibt es.

In letzter Zeit hat es in anderen Wahlkreisen einige solcher Fälle gegeben. Kurz gesagt, ist die Macht der Parteizentrale bei der Auswahl der Kandidaten größer geworden. Der Einfluss der einzelnen kôenkai im Wahlkreis ist schwächer geworden.

o Welche Anreize bestehen noch für Erbabgeordnete?

In erster Linie, dass die kôenkai, also die Basisorganisationen im Wahlkreis, übernommen werden. Deshalb ist es im Allgemeinen, normalerweise, nicht nötig, eine Basis oder Wahlkampforganisation selbst aufzubauen. Mit anderen Worten, man kann in Ruhe seinen Wahlkampf führen und sich der Arbeit im Parlament widmen. Diesen Vorteil hat man.

Die Leute, die diesen Vorteil nicht haben, müssen, selbst wenn sie die letzte Wahl gewonnen haben, schon daran denken, dass sie die nächste Wahl gewinnen müssen. Darum müssen sie noch mehr Anhänger gewinnen, im Wahlkreis Versammlungen und Veranstaltungen durchführen und verbrauchen dafür viel Energie. Deshalb haben sie dann für die Parlamentsarbeit weniger Zeit. Da haben wir also Vorteile. Wenn man allerdings so viele Wahlkämpfe geführt hat, steigt ja auch das Alter der kôenkai. Die Organisation wird älter. So ist es auch mit meiner kôenkai. Die Mitglieder der kôenkai meines Großvaters sind ja schon 80, 90 Jahre alt. Das geht natürlich nicht. Das funktioniert nicht. [Lacht.]

Ich muss das Alter der kôenkai senken, sie verjüngen. Diese Arbeit muss ich natürlich machen. Aber das ist etwas, dass mit den Erbabgeordneten nichts zu tun hat, sondern eine Situation, in der sich alle Kandidaten befinden.

o Haben Erbabgeordnete noch Vorteile gegenüber ihren Konkurrenten? Also während des Wahlkampfes?

Während des Wahlkampfes? Ja, das ist letztendlich der bekannte Name, den man hat. Der Bekanntheitsgrad. Natürlich wird das wahrscheinlich der Ruf des Vorgängers, des Vaters, sein. Zum Beispiel, wenn man Kinder hat, haben die den gleichen Familiennamen. Dann haben sie auch den Bekanntheitsgrad. Das ist ein Vorteil.

o Also das sanban-System.

Genau. Das sind also jiban [eine Basis], kanban [ein Aushängeschild], kaban [eine Tasche]. Wobei allerdings die „Tasche“ in letzter Zeit schwer zu füllen ist, nicht wahr. Also mit kaban hat das nicht allzuviel zu tun, meine ich. Es ist vor allem kanban, das einen großen Einfluss hat, ein großes Plus darstellt.

- Gibt es eine Zukunft für Erbfolge in den Wahlkreisen unter den neuen Bedingungen des politischen Systems?

Ich denke, auf der Grundlage des neuen politischen Systems wird die Zahl der Abgeordneten, die eine Nachfolge antreten, langsam abnehmen. Wie ich ja bereits sagte, nimmt der Einfluss der kôenkai ab, und der Einfluss der Parteizentrale nimmt zu. 
Wenn ich zum Beispiel meinen Sohn nachfolgen lassen wollte, aber dieser Sohn hätte nichts im Kopf oder so. [Lacht.] Oder er würde schlecht aussehen. Oder wäre ein völlig missratener Sohn, bei dem man sowieso nichts mehr retten kann...

Unter dem bisherigen Wahlsystem konnte wirklich jeder, wenn er über eine große Organisation verfügte, zumindest einmal gewinnen. Aber in den Einerwahlkreisen muss man mehr als die Hälfte [der Stimmen d.A.] bekommen. Deshalb kann man nicht gewählt werden, wenn man dumm ist.

Seitens der Parteizentrale kann also nicht mehr die Entscheidung gefällt werden: „Wenn es Ihr Sohn ist, ist es egal, was er für ein Mensch ist. Er wird aufgestellt.“ Sondern es besteht die Möglichkeit, dass die Parteizentrale sagt: „Dein Sohn wird die Wahl wahrscheinlich nicht schaffen. Lass ihn aufhören, jemand anders tritt an.“

Das heißt, die Erbabgeordneten..., also wenn alle so überragend sind, werden sie auch weiterhin eine Nachfolge antreten können. Aber ich glaube nicht, dass sie alle so gut sind, nicht wahr? Deshalb wird automatisch die Zahl der Kandidaten, die eine Nachfolge antreten, abnehmen.

- Welche Veränderungen wird es Ihrer Meinung nach bei der Kandidatenrekrutierung geben?

Wie ich schon sagte, wird die Macht der Parteizentrale zunehmen. Darüber hinaus besteht die Möglichkeit, dass Vorwahlen, die unter den Parteimitgliedern durchgeführt werden, häufiger werden. Wenn es niemanden gibt, wird bei der Kandidatenauswahl im Wahlkreis ein Aufruf gemacht. Alle Leute, die kandidieren möchten, melden sich, und die Parteizentrale wählt einen passenden Kandidaten aus. Diese Methode wird jetzt angewendet.

o Allerdings kann es ja passieren, dass sich bei einer Vorwahl unter den Parteimitgliedern nur wenige kôenkai -Mitglieder befinden oder umgekehrt unter den Mitgliedern der kôenkai nur wenige Parteimitglieder sind. Ist das nicht ein Problem?

Ja, diesen „Gap“ gibt es. Das ist sicherlich der größte Schwachpunkt der LDP. In meiner kôenkai sind nur 10\% der Mitglieder in der Partei. Deshalb gibt es nur die Möglichkeit, die Vorwahlen in kleinem Kreise durchzuführen. Wo ist dann der Nutzen von Vorwahlen? Ich glaube, dass es derzeit landesweit nur wenige Wahlkreise gibt, wo Vorwahlen von Nutzen sind.

- Gibt es Probleme, geeignete Nachwuchskräfte zu finden?

Geeignete Nachwuchskräfte?

o Ja, z.B. ausreichend junge Kandidaten?

Ach so, nun...

o Das ist vielleicht überall das gleiche Problem, aber...

Die Wahrscheinlichkeit wird immer größer, dass von der Partei Kandidaten auswählt werden, die geeignet sind, bei der Wahl ihren Gegner zu schlagen.

Natürlich gibt es auch welche, die ihre eigenen Parteifreunde attackieren, also zum Beispiel „Koizumi hat da ein paar sehr komische Sachen gesagt“ oder so und eine Gegenposition einnehmen. Dieses Problem gibt es.

Aber es werden wohl geeignete Kandidaten ausgewählt, die für die Partei Vorteile mitbringen, und die auch gegenüber ihren Gegnern Vorteile haben. Ich denke, dass sich bald die Effizienz dieses Systems 
Erbabgeordnete - Entstehung und Zukunft eines Rekrutierungspfades

zeigen wird. Aber wie ich schon sagte, wird die Macht der Parteizentrale zunehmen. Die Zahl der Erbabgeordneten wird sich verringern. Diese Veränderungen gibt es. 


\subsection{Glossar}

amakudari

天下り

chûsenkyokusei

dôshi-uchi

gakubatsu

habatsu

派閥

並立制

shôsenkyokusei

小選挙区制

hireidaihyôsei

中選挙区制

同士打ち

学閥
Netzwerke von Absolventen, v.a. der japanischen Eliteuniversitäten.

„Faktion“, engl. faction, innerparteiliche Gruppen v.a. in der LDP, die eine entscheidende Rolle bei der Verteilung von Posten und Macht spielen.

Ab 1996 für das Unterhaus angewandtes Wahlsystem, (auch „Grabensystem“) in dem ein Teil der Sitze über das Mehrheitswahlsystem in Einerwahlkreisen und ein anderer Teil in sogenannten „Blocks“ über Parteilisten vergeben wird. 
Iron Triangle

kinken seiji

金権政治

kôenkai

後援会

kosutarika-hôshiki

コスタリカ方式

oyabun - kobun 親分·子分
Das „Eisernes Dreieck“ war ein Symbol des 1955erSystems und bezeichnete die engen Verflechtungen zwischen Politik, Wirtschaft und Bürokratie.

Money-powered politics, viel kritisierter Politikstil während des 1955er-System.

„Unterstützerorganisationen“ der Kandidaten. Dabei ist zwischen so genannten „Spenden-kôenkai“ zum Sammeln von politischen Spenden und „Wählerkôenkai“ als Wählerbasis der Kandidaten zu unterscheiden. Diese kôenkai bestehen oft aus einem Netzwerk von Organisationen, von Unternehmervereinen bis Hobby-Clubs, und sind an die Person des Kandidaten gebunden.

Bei dieser Vorgehensweise wechseln sich zwei Politiker der gleichen Partei, die beide den gleichen Einerwahlkreis für sich beanspruchen in der Kandidatur dort ab. Tritt zunächst Politiker A als Direktkandidat an, weicht Politiker B auf die Parteiliste aus. Bei der nächsten Wahl verfahren beide umgekehrt. “495

“Elternteil“ - „Kindteil”, Bezeichnung von so genannten Patron-Klient-Beziehungen in japanischen Gruppen, die durch gegenseitige Fürsorge bzw. Loyalität geprägt sind.

Policy Affairs Research Council, zentrales Organ der LDP, das während der LDP-Regierungszeit jede Kabinettsvorlage vor der Eingabe in das Parlament prüfte. Das PARC verfügt über Unterausschüsse zu ein-

${ }^{495}$ Klein, Axel: A.a.O., S. 284. 
zelnen Politikfeldern, die von einflussreichen zoku giin geführt werden.

sâbissu gassen サービス合戦 „Service-Schlachten“, in denen die Kandidaten die Wähler mit „Wohltaten“, kleinen Geschenken und Gefälligkeiten umwerben.

sanban

さんばん

Drei Dinge, über die ein aussichtsreicher Kandidat in einem Wahlkampf verfügen muss. Dazu zählen

jiban

地盤 eine feste Basis im Wahlkreis, bestehend aus den kôenkai und lokalen Netzwerken,

kaban

鞄

eine Tasche mit Wohltaten für die Anhängerschaft und den Wahlkreis (Subventionen etc.) und

kanban

看板 ein Aushängeschild, d.h. ein bekanntes Gesicht bzw. ein bekannter Name.

sangiin

参議院

„Beraterkammer“, japanisches Oberhaus, das eigentlich als Ort überparteilicher Beratungen konzipiert war und geringeren politischen Einfluss als das Unterhaus besitzt.

senpai - kôhai

先輩· 後輩

„Älterer“ - „Jüngerer“. In der japanischen Gesellschaft häufig anzutreffende Hierarchie, die auf Seniorität basiert.

seshûdaigishi

世襲代議士

„Erbabgeordnete“, die ein Mandat in

niseigiin

二世議員

zweiter Generation oder

sanseigiin

seijikazoku

三世議員

dritter Generation (oder höher) wahrnehmen und somit aus Politikerfamilien stammen.

政治家族

sewanin

世話人

Personen, die Führungsaufgaben in den kôenkai übernehmen. 
shôkaisha

shûgiin

Sôka Gakkai

tarento

zoku giin
紹介者

衆議院

創価学会

タレント

ともだおれ
„Leute die vorgestellt wurden“. Mit den kôenkai eines Kandidaten lose verbundene Wähler.

Japanisches Unterhaus.

„Wissenschaftliche Gesellschaft zur Schaffung von Werten“. 1930 von Tsunesaburô Makiguchi gegründete Sekte, die in der Nichiren-Schule ihre Wurzeln hat. Sie betont die sozialpolitischen Verpflichtungen der Religion und ist relativ nationalistisch. ${ }^{496}$

“Talente”, Abgeordnete, die vor ihrer Karriere Prominente, Sportler etc. waren und v.a. aufgrund ihrer Popularität ins Parlament gewählt werden.

„Gemeinsames Fallen“ von Kandidaten einer Partei, die unter dem alten Wahlsystem im selben Wahlkreis gegeneinander antraten. Stellte eine Partei zu viele Kandidaten auf, nahmen sie sich gegenseitig die Stimmen weg, so dass keiner der aufgestellten Kandidaten ins Parlament einzog.

Erfahrene Politiker der LDP, die zu einem „Stamm“ gezählt werden, d.h. die sich auf ein bestimmtes Politikfelder spezialisiert haben. Sie übernehmen nicht nur die Führung in Ausschüssen von Partei und Parlament, sondern sind aufgrund ihrer guten Kontakte auch als Vermittler zwischen Bürokratie und Wirtschaft gefragt und verfügen über einen guten Zugang zu Ressourcen.

\footnotetext{
${ }^{496}$ Ehrhard, Franz-Karl; Fischer-Schreiber, Ingrid: Das Lexikon des Buddhismus, 3. Auflage, Goldmann Verlag, München 1995, S. 160; 206.
} 


\subsection{Abkürzungsverzeichnis (Parteien)}

(Fett gedruckt die Parteien, die im Sommer 2005 im Unterhaus vertreten waren.)

\begin{tabular}{|c|c|c|}
\hline CGP & $\begin{array}{l}\text { Clean Government Party } \\
\text { (1964 ) }\end{array}$ & $\begin{array}{l}\text { (New) Kômeitô } \\
\text { 公明党 }\end{array}$ \\
\hline DPJ & $\begin{array}{l}\text { Democratic Party of Japan } \\
\text { (1996 ) }\end{array}$ & $\begin{array}{l}\text { Minshutô } \\
\text { 民主党 }\end{array}$ \\
\hline DSP & $\begin{array}{l}\text { Democratic Socialist Party } \\
(1960-1994)\end{array}$ & $\begin{array}{l}\text { Minshu-Shakaitô (Minshatô) } \\
\text { 民主社会党 }\end{array}$ \\
\hline JNP & $\begin{array}{l}\text { Japan New Party } \\
(1992-1994)\end{array}$ & $\begin{array}{l}\text { Nihon Shintô } \\
\text { 日本新党 }\end{array}$ \\
\hline JRP & $\begin{array}{l}\text { Japan Renewal Party } \\
\text { (1993-1994) }\end{array}$ & $\begin{array}{l}\text { Shinseitô } \\
\text { 新生党 }\end{array}$ \\
\hline KPJ/JPC & $\begin{array}{l}\text { Japan Communist Party } \\
(1922 \sim)\end{array}$ & $\begin{array}{l}\text { Kyôsantô } \\
\text { 共産党 }\end{array}$ \\
\hline LDP & $\begin{array}{l}\text { Liberal Democratic Party } \\
\text { (1955 ) }\end{array}$ & $\begin{array}{l}\text { Jiyûminshutô (Jimintô) } \\
\text { 自由民主党 }\end{array}$ \\
\hline LP & $\begin{array}{l}\text { Liberal Party } \\
(1998 \sim 2003)\end{array}$ & $\begin{array}{l}\text { Jiyûtô } \\
\text { 自由党 }\end{array}$ \\
\hline NCP & $\begin{array}{l}\text { (New) Conservative Party } \\
(2000 \sim)\end{array}$ & $\begin{array}{l}\text { Hoshutô } \\
\text { 保守党 }\end{array}$ \\
\hline NFP & $\begin{array}{l}\text { New Frontier Party } \\
(1994-1998)\end{array}$ & $\begin{array}{l}\text { Shinshintô } \\
\text { 新進党 }\end{array}$ \\
\hline NPS & $\begin{array}{l}\text { New Party Sakigake } \\
(1993-2001)\end{array}$ & $\begin{array}{l}\text { Shintô Sakigake } \\
\text { 新党さきがけ }\end{array}$ \\
\hline SPJ / SDPJ & $\begin{array}{l}\text { Socialist Party of Japan / Socialdemoc- } \\
\text { ratic Party of Japan } \\
(1945 \sim)\end{array}$ & $\begin{array}{l}\text { Shakaitô / Shakai-Minshutô } \\
\text { (Shamintô) } \\
\text { 社会党 / 社会民主党 }\end{array}$ \\
\hline
\end{tabular}


Nach der Unterhauswahl 2005 sind noch weitere, kleinere Gruppierungen im japanischen Parlament vertreten. 


\subsection{Literatur}

\subsubsection{Monographien}

Arnim, Hans Herbert von: Fetter Bauch regiert nicht gern. Die politische Klasse selbstbezogen und abgehoben, Knaur, München 1999.

Asahi Shinbun: Senkyô-Taikan 1996, Asahi Shinbun-sha, Tôkyô 1996.

\section{朝日新聞 「選挙大観 1996 」朝日新聞社 東京 1996 . (Asahi Shinbun: Ein Überlick über die Wahl 1996.)}

Atteslander, Peter: Methoden der empirischen Sozialforschung, 8. bearb. Auflage, de Gruyter, Berlin 1995.

Beasley, W.G.: The Rise of Modern Japan, St. Martin’s Press, New York 1990.

Beyme, Klaus von: Die politische Klasse im Parteienstaat, 2. Aufl., Suhrkamp, Frankfurt/M. 1995.

Blechinger, Verena: Politische Korruption in Japan, Institut für Asienkunde, Hamburg 1998.

Bruns, Gabriele: Die japanische Demokratie. Kieler Schriften zur politischen Wissenschaft 10, Peter Lang Verlag, Frankfurt/M. 1999.

Curtis, Gerald L.: The Logic of Japanese Politics: Leaders, Institutions and the Limits of Change, Columbia University Press, New York 1999.

Ehrhard, Franz-Karl; Fischer-Schreiber, Ingrid: Das Lexikon des Buddhismus, 3. Auflage, Goldmann Verlag, München 1995.

Feldman, Ofer: Politics and the News Media in Japan, University of Michigan Press, Ann Arbor 1996.

Flick, Uwe: Qualitative Forschung. Theorie, Methoden, Anwendung in Psychologie und Sozialwissenschaften, 4. Auflage, Rowohlt Verlag, Hamburg 1999.

Flick, Uwe: Qualitative Sozialforschung. Eine Einführung, 6. Auflage, Rowohlt Verlag, Hamburg 2002.

Foreign Press Center Japan (Hg.): Changing Japanese Politics. “About Japan” Series, Nr. 26, 2. überarb. Aufl., Foreign Press Center Japan, Tôkyô 2003.

Foreign Press Center Japan (Hg.): Facts and Figures of Japan 2003, Foreign Press Center Japan, Tôkyô 2003.

Foreign Press Center Japan (Hg.): The Diet, Election and Political Parties. “About Japan” Series, Nr. 13, 3. Aufl., Foreign Press Center Japan, Tôkyô 1995.

Hanai, Hirotada: Seiji-no Shikumi, Daiyamondo-sha, Tôkyô 1996.

花井ひろただ「政治のしくみ」ダイヤモンド社＼cjkstart東京1996. (Hanai, Hirotada: Mechanismen der Politik.) 
Hartmann, Jürgen: Politik und Gesellschaft in Japan, USA und Westeuropa: Ein einführender Vergleich, Campus Verlag, Frankfurt/M. 1983.

Hirano, Hiroshi; Kôno, Masaru: Nihon seijiron, Nihon Keizaihyôronsha, Tôkyô 2003. 平野 河野「日本政治論」日本経済評論社東京 2003 . (Hirano, Hiroshi; Kôno, Masaru: Theorien japanischer Politik.)

Ichikawa Taiichi: Seshûdaigishi-no kenkyû, Nihon Keizai Shinbun-sha, Tôkyô 1990. 市川太－「世襲代議士の研究」日本経済新聞社 東京1990. (Ichikawa, Taiichi: Forschung zu Erbabgeordneten.)

Ishida, Hiroshi: Social Mobility in Contemporary Japan, Macmillan Press, Basingstoke 1993.

Iwanaga, Kazuki: Democracy and Electoral Systems in Japan. A Comparative Perspective, Center for Pacific Asia Studies at Stockholm University, Stockholm 1995.

Johnson, Chalmers: Japan. Who governs? The Rise of the developmental State, Norton, New York 1995.

Johnson, Stephen: Opposition Politics in Japan. Strategies under a One-Party dominant Regime, Routledge, London 2000.

Kagaya, Katsuo: Asahi kîwâdo. Bessatsu seiji, kempô, Tôkyô 2000.

加賀勝雄「朝日キーワード別冊一政治・憲法」朝日新聞社 東京 2000 . (Kagaya, Katsuo: Asahi Keywords. Sonderband Politik und Verfassung.)

Kasawara, Morio: Asahi kîwâdo. Bessatsu seiji, kempô, Tôkyô 1997. 笠原もりお「朝日キーワード別冊一政治・憲法」朝日新聞社 東京 1997 . (Kasawara Morio: Asahi Keywords. Sonderband Politik und Verfassung.)

Katô, Shûjirô: Seiji-no shikumi. Nihon-ha kô natte iru, PHP Kenkyûsho, Tôkyô 1995. 加藤秀治郎「政治のしくみ 日本はこうなっている」PHP 研究所 東京 1995. (Katô, Shûjirô: Mechanismen der Politik. So ist Japan.)

Kenji, Hayao: The Japanese Prime Minister and Public Policy, University of Pittsburgh Press, Pittsburgh 1993.

Kerbo, Harold R.: Who rules Japan? The inner circle of economic and political power, Praeger Publishers, London 1995.

Klein, Axel: Das Wahlsystem als Reformobjekt. Eine Untersuchung zu Entstehung und Auswirkung politischer Erneuerungsversuche am Beispiel Japan. Bonner Japanforschungen Bd. 18, Bier’sche Verlagsanstalt, Bonn 1998.

Koh, Byung Chol: Japan’s Administrative Elite, University of California Press, Berkeley 1989. 
Kuckartz, Udo: Computergestützte Analyse qualitativer Daten: Eine Einführung in Methoden und Arbeitstechniken, Westdeutscher Verlag, Opladen 1999.

Kunio, Yoshihara: Sogo Shôsha. The vanguard of the Japanese economy, Oxford 1982.

Lamnek, Siegfried: Qualitative Sozialforschung. Band 2: Methoden und Techniken.

Psychologische Verlagsunion, München 1989.

Masumi, Junnosuke: Contemporary Politics in Japan, University of California Press, Berkeley 1995.

Masumi, Junnosuke: Postwar Politics in Japan 1945-1955, University of California Press, Berkeley 1985.

Masuzoe, Yôichi: Naikaku-Sôridaijin - Sono Rikiryô to Shishitsu-no mi-kiwamekata, Kootobukurain, Tôkyô 2002.

忡添要一「内閣総理大臣 - その力量と資質の見極め方」株式会社コオトブッ クライン 東京 2002 .

(Masuzoe, Yôichi: Der Ministerpräsident - Ein Forschungsansatz seiner Begabung und Qualität.)

Nakane, Chie: Die Struktur der japanischen Gesellschaft, Suhrkamp, Frankfurt/M. 1992.

Norris, Pippa; Lovenduski, Joni: Political Recruitment. Gender, race and class in the British parliament, Cambridge University Press, Cambridge 1995.

Okada, Kenji: Seijiseido-no Shikumi, Kabushikikaisha Nashime-sha, Tôkyô 1999. 岡田憲治「政治制度のしくみ」株式会社ナシメ社＼cjkstart東京１９９９． (Okada, Kenji: Mechanismen des politischen Systems.)

Park, Cheol Hee: Daigishi-no tsukurarekata. Shosenkyoku-no senkyo-senryaku, Bunshushinsho, Tôkyô 2000.

バク・チョルヒー「代議士のつくられ方 小選挙区の選挙戦略」文春新書

東京 2000 .

(Park Cheol-Hee: Wie Abgeordnete gemacht werden. Wahlkampfstrategien in den Einerwahlkreisen.)

Patzelt, Werner J.: Abgeordnete und ihr Beruf: Interviews - Umfrage - Analysen, Akad. Verlag, Berlin 1995.

Pharr, Susan J.: Political Women in Japan: The Search for a Place in political Life, University of California Press, Berkeley 1981.

Pharr, Susan J.; Krauss Ellis S.: Media and Politics in Japan, University of Hawaii Press, Honolulu 1996.

Ramseyer, J. Mark; MacCall Rosenbluth, Frances: Japan’s Political Marketplace, Harvard 1993. 
Rothacher, Albrecht: The Japanese Power Elite, St. Martins Press, New York 1993.

Saitô, Keiko: Seikanyôran, Vol. 19, No. 45, Seisakujihô Ai Pi, Tôkyô 2001.

齋藤京子「政官要覧 2001 、Vol.19、No.45」セイサクジホウ・ア イ・ピイ 東京 2001 .

(Saitô, Keiko: Führer durch Politik und Bürokratie.)

Saitô, Keiko: Seikanyôran, Vol. 20, No. 47, Seisakujihô Ai Pi, Tôkyô 2002.

齋藤京子「政官要覧 2002 、Vol. 20 、No.4 7 セセイサクジホウ・ア イ・ピイ 東京 2002 .

(Saitô, Keiko: Führer durch Politik und Bürokratie.)

Saitô, Keiko: Seikanyôran, Vol. 22, No. 50/2003, Seisakujihô Ai Pi, Tôkyô 2004.

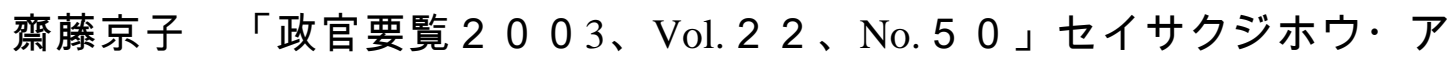
イ・ピイ 東京 2004.

(Saitô, Keiko: Führer durch Politik und Bürokratie.)

Schlesinger, Jacob M.: Shadow Shoguns. The Rise and Fall of Japan's Postwar Political Machine, $2^{\text {nd }}$ Edition, Standford University Press, Stanford 1999.

Seifert, Wolfgang: Gewerkschaften in der japanischen Politik von 1970 bis 1990. Der dritte Partner? Westdeutscher Verlag, Opladen 1997.

Shiratori, Rei: Shôsenkyokusei-de Seiji-ha dô naru-ka, Libati-Shobô, Tôkyô 1995.

白鳥令「小選挙区制で政治はどうなるか」リバティ書房 東京 1995 .

(Shiratori Rei: Wie entwickelt sich die Politik unter dem Einerwahlsystem?)

Tôkyô Shinbun: Dai 40 kai shûgiinsen dêtabukku, Tôkyô Shinbun Senkyohôdôhonbu, Tôkyô 1993.

東京新聞「第 40 回衆院選データブック」東京新聞選挙報道本部＼cjkstart東京 19 93.

(Tokyo Shinbun: Datenhandbuch zur 40. Unterhauswahl.)

Uji, Toshihiko: Shushô retsuden. Itô Hirobumi kara Koizumi Junichirô made, Tôkyô Shoseki Kabushikikaisha, Tôkyô 2001.

宇治敏彦「首相列伝 伊藤博文から小泉純一郎まで」東京書籍株式会社 東 京 20001.

(Uji, Toshihiko: Die Biographien der japanischen Ministerpräsidenten. Von Itô Hirobumi bis Koizumi Junichirô, Tôkyô 2001.)

Wada, Hideki: Nagatachô “seishinbunseki” hôkoku, Shôgakukanbunko, Tôkyô 2002. 和田秀樹「永田町『精神分析』報告」小学館文庫 東京 2002 . 
(Wada, Hideki: Eine psychologische Analyse von Nagatachô. )

Wada, Junichiro: The Japanese election System: three analytical perspectives, Routledge, London 1996.

Weber, Max: Politik als Beruf [1919], Reclam, Stuttgart 1992.

Wehling, Hans-Georg: Japan. Kohlhammer Taschenbücher Bd. 1071: Bürger im Staat, Kohlhammer, Stuttgart 1985.

White, James W.: The Sokagakkai and Mass Society, Stanford University Press, Stanford 1970.

Woodall, Brian: Japan under construction: corruption, politics and public work, University of California Press, Berkeley 1996.

Wray, Harry; Conroy, Hilary (Hrsg.): Japan examined: Perspectives on modern Japanese history, University of Hawaii Press, Honolulu 1983.

Yamaguchi, Kazuto: Das parlamentarische System Japans. Wissenschaftliche Dienste des Deutschen Bundestages Nr. 128, Bonn 1998.

Yomiuri Shinbun: Gekihen-no seijisentaku. 89 san 90 shûgiinsen tettei bunseki, Yomiuri Shinbun-sha, Tôkyô 1990.

読売新聞「激変の政治選択。8 9 参 90 衆院選徹底分析」読売新聞社編 東 京 1990 .

(Yomiuri Shinbun: Politische Auswahl im Umbruch. Eine umfassende Analyse der Wahlen zum Oberhaus 1989 und zum Unterhaus 1990.)

Yoshino, Takashi; Imamura, Osamu; Tanitô, Etsushi (Hrsg.): Dare-ga Seijika-ni naru-no-ka - Kôhoshaerabi-no Kokusaihikaku, Waseda-Daigaku Shuppanbu, Tôkyô 2001. 吉野孝、今村治、谷藤悦史「誰が政治家になるのか - 候補者選びの国際比 較」早稲田大学出版部 東京 2001 .

(Yoshino, Takashi et.al.: Wer wird Politiker? Ein internationaler Vergleich der Kandidatenauswahl.)

\subsubsection{Aufsätze}

Allinson, Gary D.: The Structure and Transformation of Conservative Rule, in: Gordon, Andrew (Hrsg.): Postwar Japan as History, University of California Press, Berkeley 1993, S. 123-144.

Beyme, Klaus von: Der Begriff der politischen Klasse - eine neue Dimension der Elitenforschung? In: Politische Vierteljahresschrift, Jg. 33, Heft 1/1992, S. 2-31.

Beyme, Klaus von: Der Führungsanspruch der politischen Klasse, in: Bude, Heinz; Schleissing, Stephan: Junge Eliten: Selbständigkeit als Beruf, Kohlhammer, Stuttgart 1997, S. 39-50. 
Blechinger, Verena: Auf dem Weg zu „sauberer Politik“ und transparenten Strukturen? Korruption und Selbstreinigung in der japanischen Politik, in: Borchert, Jens; Leitner, Sigrid; Stolz, Klaus (Red.): Politische Korruption. Jahrbuch für Europa- und Nordamerika-Studien 3, Leske+Budrich, Opladen 2000, S. 145-184.

Blechinger, Verena: Politik und Familienbande - „Erbabgeordnete“ im japanischen Parlament, in: Nachrichten der Gesellschaft für Natur- und Völkerkunde Ostasiens, Nr. 159/160, 1997, S. 71-87.

Blechinger, Verena: Politische Reformen in Japan - Auswirkungen und Perspektiven, in: Bosse, Friederike; Köllner Patrick (Hrsg): Reformen in Japan, Mitteilungen des Instituts für Asienkunde Nr. 337, Institut für Asienkunde, Hamburg 2001, S. 89-110.

Borchert, Jens: Politik als Beruf: Die politische Klasse in westlichen Demokratien, in: Borchert, Jens (Hg.): Politik als Beruf. Die politische Klasse in westlichen Demokratien, Leske+Budrich, Opladen 1999, S. 7-39.

Borchert, Jens: Pathways to Professionalism: Institutional Context and the Emergence of a Political Class in the U.S. and Germany, Unveröff. Mskr., APSA, Washington, D.C. 1997.

Borchert, Jens; Golsch, Lutz: Die politische Klasse in westlichen Demokratien: Rekrutierung, Karriereinteressen und institutioneller Wandel, in: Politische Vierteljahresschrift, Nr. 35/1995, S. 609-629.

Bouisseau, Jean-Marie: Organizing One’s Support Base under the SNTV: The Case of Japanese Koenkai, in: Grofman, Bernard et.al. (Hg.): Elections in Japan, Korea and Taiwan under the Single Non-Transferable Vote, The University of Michigan Press 1999, S. 87-120.

Campbell, John Creighton: Democracy and Bureaucracy in Japan, in: Ishida, Takeshi; Krauss, Ellis S. (Hg.): Democracy in Japan, 3.Auflage, University of Pittsburgh Press, Pittsburgh 1994, S. 113-137.

Christensen, Raymond V.: The New Japanese Election System, in: Pacific Affairs, No. 69/1996, S. 49-70.

Christensen, Raymond V.: The Effect of electoral Reform on Campaign Practices in Japan. Putting New Wine into Old Bottles, in: Asian Survey, No. 38/1998, S. 986-1004.

Colignon, Richard; Usui, Chikako: The resilience of Japan's iron triangle, in: Asian Survey, Vol. 41, No. 5/2001, S. 865-890.

Cox, Gary W.; McCall Rosenbluth, Frances: The structural determinants of electoral cohesiveness: England, Japan, and the United States, in: Cowhey, Peter F.; McCubbins, Matthew D.: Structure and Policy in Japan and the U.S., Cambridge University Press, Cambridge 1995, S. 19-80.

Cox, Gary W.; Niou, Emerson: Seat Bonuses under the Single Non-Transferable Vote 
System: Evidence from Japan and Taiwan, in: Grofman, Bernard et.al. (Hg.): Elections in Japan, Korea and Taiwan under the Single Non-Transferable Vote, The University of Michigan Press 1999, S. 354-374.

Derichs, Claudia; Kerbo, Harold: Japan. Politische Karriere zwischen Ministerialbürokratie und Erbhöfen, in: Borchert, Jens (Hrsg.): Politik als Beruf. Die politische Klasse in westlichen Demokratien. Reihe Europa- und Nordamerika-Studien Bd. 5, Leske + Budrich, Opladen 1999, S. 283-201.

Derichs, Claudia; Thompson, Mark: Dynastien und politische Führerinnen in Asien. Project Discussion Paper No. 1/2003, Universität Duisburg Essen und Universität ErlangenNürnberg, 2003.

Dore, R.P.: Rural Conservatism, in: : Livingston, John et. al. (Hg.): Postwar Japan. 1945 to the Present, Pantheon Books, New York, S.331-335.

Dore, Ronald: Japan's Reform Debate: Patriotic Concern or Class Interest? Or Both? In: Journal of Japanese Studies, Vol. 25, No. 1/1999, S. 65-89.

Farley, Maggie: Japans Press and the Politics of Scandal, in: Farley, Maggie; Krauss, Ellis S.: Media and Politics in Japan, University of Hawaii Press, Honolulu 1996, S. 133164.

Flick, Uwe; Kardoff, Ernst von; Steinke, Ines: Was ist qualitative Forschung? Einleitung und Überblick, in: Flick, Uwe; von Kardoff, Ernst; Steinke, Ines (Hg.): Qualitative Forschung. Ein Handbuch, Rowohlt, Reinbek 2000, S. 13-29.

Schmidt, Christiane: Analyse von Leitfadeninterviews, in: Flick, Uwe; von Kardoff, Ernst; Steinke, Ines (Hg.): Qualitative Forschung. Ein Handbuch, Rowohlt, Reinbek 2000, S. 447-456.

Fowler, Linda: Theories of Recruitment in Comparative Perspective, Unveröff. Mskr., IPSA, Berlin 1994.

Fukui Haruhiro: Japan, in: Norris, Pippa: Passages to power: legislative recruitment in advanced democracies, Cambridge University Press, Cambridge 1997, S. 98-114.

Fukui, Haruhiro; Fukai, Shigeko: Pork Barrel Politics, Networks, and local economic Development in Contemporary Japan, in: Asian Survey, Vol. 36, No. 3/1996, S. 268286.

Hallier, Christoph: Politikverdrossenheit in Japan, in: Pohl, Manfred; Wieczorek, Iris (Hg.): Japan 2000/2001, Institut für Asienkunde Hamburg 2001, S. 53-81.

Hrebenar, Ronald R.: The Changing Postwar Party System, in: Hrebenar, Ronald J: Japan’s new party system, Westview Press, Oxford 2000, S. 1-36.

Hrebenar, Ronald J.: Rules of the Game: The Impact of the Electoral System on Political Parties, in: Hrebenar, Ronald J: Japan’s new party system, Westview Press, Oxford 
2000, S. 37-58.

Hrebenar, Ronald J.: The Liberal Democratic Party: Still the Most Powerful Party in Japan, in: Hrebenar, Ronald J: Japan’s new party system, Westview Press, Oxford 2000, S. 85-148.

Hrebenar, Ronald J.: The New Parties of the Second Party System, in: Hrebenar, Ronald J: Japan’s new party system, Westview Press, Oxford 2000, S. 149-166.

Hrebenar, Ronald J.: The Komeito Returns: The Party of "Buddhist Democracy", in: Hrebenar, Ronald J: Japan's new party system, Westview Press, Oxford 2000, S. 167207.

Inoguchi, Takashi: A Step Toward One-Party Predominance: Japan's General Election of 20 October 1996, in: Government and Opposition, Vol. 32, No. 1/1997, S. 48-64.

Ishibashi, Michihiro; Reed, Steven R.: Second-Generation Diet Members and Democracy in Japan, in: Asian Survey, Vol. 32, No. 4/1992, S. 366-379.

Ishida, Takeshi; Krauss, Ellis S.: Democracy in Japan: Issues and Questions, in: Ishida, Takeshi; Krauss, Ellis S. (Hg.): Democracy in Japan, 3.Auflage, University of Pittsburgh Press, Pittsburgh 1994, S. 3-16.

Jain, Purnendra C.: Party Politics at the Crossroads, in: Jain, Purnendra C.; Inoguchi, Takashi: Japanese Politics Today. Beyond Karaoke Democracy? St. Martin’s Press, New York 1997, S. 11-29.

Jain, Purnendra C.; Todhunter, Maureen: The 1996 General Election: Status Quo or Step forward? In: Jain, Purnendra C.; Inoguchi, Takashi: Japanese Politics Today. Beyond Karaoke Democracy? St. Martin’s Press, New York 1997, S. 224-239.

Kataoka, Masaaki: Changing local Politics: Party Realignment and growing Competition, in: Jain, Purnendra C.; Inoguchi, Takashi: Japanese Politics Today. Beyond Karaoke Democracy? St. Martin’s Press, New York 1997, S. 206-223.

Kato, Junko: When the Party Breaks Up: Exit and Voice among Japanese Legislators, in. American Political Science Review, Vol. 93, No. 4/1998, S. 857-870.

Köllner, Patrick: Drei Jahre nach den politischen Reformen - Eine Zwischenbilanz. Teil 1: das Wahlgesetz und seine Auswirkungen, in: Japan aktuell. Wirtschaft. Politik. Gesellschaft, Heft April/1998, S. 167-177.

Köllner, Patrick: Drei Jahre nach den politischen Reformen - Eine Zwischenbilanz. Teil 2: Die Finanzierung politischer Aktivitäten, in: Japan aktuell. Wirtschaft. Politik. Gesellschaft, Heft Juni/1998, S. 272-283.

Köllner, Patrick: Informelle Elemente in der japanischen Politik. Teil 1: Faktionen, in: Japan aktuell. Wirtschaft. Politik. Gesellschaft, Heft Februar 1999, S. 65-75.

Köllner, Patrick: Informelle Elemente in der japanischen Politik. Teil 2: 
Unterstützervereinigungen und Erbabgeordnete, in: Japan aktuell. Wirtschaft. Politik. Gesellschaft, Heft April/1999, S. 165-173.

Köllner, Patrick: Parteienfinanzierung in Japan: Regulierungsmechanismen und Anpassungsstrategien, in: Japan aktuell. Wirtschaft. Politik. Gesellschaft, Heft April/2000, S. 147-158.

Köllner, Patrick: Faktionalismus in politischen Parteien: Der japanische Fall aus konzeptioneller und komparativer Perspektive, in: Seifert, Wolfgang; Weber, Claudia (Hg.): Japan im Vergleich, Iudicum, München 2002, S. 281-306.

Köllner, Patrick: Zwischen Konflikt, Kohäsion und parteiübergreifender Kooperation: Probleme und Perspektiven der Demokratischen Partei Japans, in: Japan aktuell. Wirtschaft. Politik, Heft Februar/2003, S. 55-63.

Krauss, Ellis S.: Politics and the Policymaking Process, in: Ishida, Takeshi; Krauss, Ellis S. (Hg.): Democracy in Japan, 3.Auflage, University of Pittsburgh Press, Pittsburgh 1994, S. 39-64.

Krauss, Ellis S.: Pekkanen, Robert: Explaining Party Adaption to Electoral Reform. The Discreet Charme of the LDP? In: Journal of Japanese Studies, Vol. 30, No. 1/2004, S. 134.

Krauss, Ellis S.; Nyblade, Benjamin: "Presidentialization” in Japan? The Prime Minister, Media and Elections in Japan, in: British Journal of Political Studies, Vol. 35, No. 2/2005, S. 357-368.

Kurtz, Donn M.: First Families in Japan, Mexico and the United States: 1946-2000, in: Comparative Sociology, Vol. 42, No. 5 / 2001, S. 461-478.

MacDougall, Terry E.: Democracy and Local Government in Postwar Japan, in: Ishida, Takeshi; Krauss, Ellis S. (Hg.): Democracy in Japan, 3.Auflage, University of Pitts burgh Press, Pittsburgh 1994, S.139-169.

Masayama Mikitaka: Seijika, seitô, in: Hirano, Hiroshi; Kôno, Masaru: Nihon seijiron, Nihon Keizaihyôronsha, Tôkyô 2003, S. 49-72.

増山幹高「政治家・政党」平野河野『日本政治論』日本経済評論社 東京 $2003, p .49-72$.

(Masayama Mikitaka: Politiker und Parteien, in: Hirano, Hiroshi; Kôno, Masaru: Theorien japanischer Politik.)

Muramatshu, Michio; Krauss, Ellis S.: The Conservative Policy Line and the Development of Patterned Pluralism, in: Yamamura Kozo; Yasuba Yasukichi (Hg.): The Political Economy of Japan. Volume 1. The Domestic Transformation, Stanford University Press, Stanford 1987, S. 516-554.

Norris, Pippa: theories of recruitment, in: Norris, Pippa: Passages to power: legislative recruitment in advanced democracies, Cambridge University Press, Cambridge 1997, S. 1-14. 
Norris, Pippa: Through the Eye of the Needle: Comparative Political Recruitment in Western Democracies, Unveröff. Mskr., APSA, Washington 1993.

Otake, Hideo: Forces for Political Reform: The Liberal Democratic Party’s Young Reformers and Ozawa Ichirô, in: Journal of Japanese Studies, Vol. 22, No. 2/1996, S. 270294.

Pempel, T.J.: Japan's Creative Conservatism. Continuity under Challenge, in: Castles, Francis G.: The Comparative History of Public Policy, Polity Press, Cambridge 1989, S. 149-191.

Pempel, T.J.: Prerequisites for Democracy: Political and Social Institutions, in: Ishida, Takeshi; Krauss, Ellis S. (Hg.): Democracy in Japan, 3.Auflage, University of Pittsburgh Press, Pittsburgh 1994, S. 17-37.

Pippa, Norris: Conclusion: comparing passages to power, in: Norris, Pippa: Passages to power: legislative recruitment in advanced democracies, Cambridge University Press, Cambridge 1997, S. 209-231.

Pohl, Manfred: Demokratie auf Japanisch, in: Wehling, Hans Georg: Japan. Kohlhammer Taschenbücher, Bd. 1071, Stuttgart 1985, S. 80-101.

Pohl, Manfred: Die politische Kultur Japans, in: Barloewen, Constantin von; Werhahn-Mees, Kai (Hg.): Japan und der Western. Band 3: Politik, Kultur und Gesellschaft, Frankfurt/M. 1986, S. 19-37.

Reed, Steven R.; Bolland, John M.: The Fragmentation Effect of SNTV in Japan, in: Grofman, Bernard et.al. (Hg.): Elections in Japan, Korea and Taiwan under the Single Non-Transferable Vote, The University of Michigan Press 1999, S. 211-226.

Schaede, Ulrike: The “Old Boy” Network and Government-Business Relationships in Japan, in: Journal of Japanese Studies, Vol. 21, No. 2/1995, S. 293-317.

Seligmann, Albert L.: Japan’s new electoral system, in: Asian Survey, Vol. 37, No. 5/1997, S. 409-428.

Steel, Gill: Gender and Voting Preferences in Japanese Lower House Elections, in: Japanese Journal of Political Science, Vol. 4, Part 1, May 2003, S. 1-39.

Stockwin, J.A.A.: Parties, Politicians and the Political System, in: Stockwin, J.A.A.: Dynamic and Immobilist Politics in Japan, University Press of Hawaii, Honolulu 1989, S. 22-53.

Stockwin, J.A.A.: Political Parties and Political Opposition, in: Ishida, Takeshi; Krauss, Ellis S. (Hg.): Democracy in Japan, 3.Auflage, University of Pittsburgh Press, Pittsburgh 1994, S. 89-111.

Stockwin, J.A.A.: Reforming Japanese Politics: Highway of Change or Road to Nowhere? In: Jain, Purnendra C.; Inoguchi, Takashi: Japanese Politics Today. Beyond Karaoke Democracy? St. Martin’s Press, New York 1997, S. 75-91. 
Stockwin, J.A.A.: The Socialist Democratic Party (Formerly Japan Socialist Party): A Turbulent Odysse, in: Hrebenar, Ronald J: Japan’s new party system, Westview Press, Oxford 2000, S. 209-252.

Tanaka, Aiji: Nihon, in: Yoshino, Takashi; Imamura, Osamu; Tanitô, Etsushi (Hrsg.): Darega Seijika-ni naru-no-ka - Kôhoshaerabi-no Kokusaihikaku, Waseda-Daigaku Shuppanbu, Tôkyô 2001, S. 3-32.

田中愛治「日本」吉野孝、今村治、谷藤悦史「誰が政治家になるのか - 候補 者選びの国際比較」早稲田大学出版部 東京 $2001 、 p .3-32$.

(Tanaka, Aiji: Japan, in: Yoshino, Takashi et.al.: Wer wird Politiker? Ein internationaler Vergleich der Kandidatenauswahl.)

Woodall, Brian: The Politics of Reform in Japan's Lower House Electoral System, in: Grofman, Bernard et.al. (Hg.): Elections in Japan, Korea and Taiwan under the Single Non-Transferable Vote, The University of Michigan Press 1999, S. 23-50.

Yoshino, Takashi; Imamura, Osamu: Nihon: Yôyaku hajimatta jôsei-no seiji-shinshutsu, in: Imamura Isamu (Hrsg.): Dare-ga seijika-ni naru-no-ka, Waseda Daigaku Shuppan-bu, Tôkyô 2001, S. 147-177.

吉野孝、今村浩「日本：ようやく始まった女性の政治進出」吉野孝、今村 治、谷藤悦史「誰が政治家になるのか - 候補者選びの国際比較」早稲田大学 出版部 東京 $2001 、 \mathrm{p} .147-177$.

(Yoshino, Takashi, Imamura, Osamu: Japan: Allmählich hat das Vorrücken der Frauen in der Politik begonnen, in: Yoshino, Takashi; Imamura, Osamu; Tanitô Etsushi (Hrsg.): Wer wird Politiker?)

\subsubsection{Zeitungs-/Zeitschriftenartikel}

Asahi Shinbun: Kônin meguri kakuha shinogi, in: Asahi Shinbun, 08.06.2000, S. 4.

Asahi Shinbun: Sôkaku-ni ,taika issô’ atsuryoku, in:Asahi Shimbun, 28.06.2000, S. 4.

朝日新聞「総閣に『滞貨一掃』圧力」朝日新聞 2000 年 6 月 28 日、 p. 4 .

(Asahi Shinbun: Der Druck auf die Regierung, die Postenanhäufung zu beseitigen.)

Asahi Shinbun: Niokuhassenman-en jimin-wa gengaku, in:Asahi Shimbun, 28.06.2000, S. 4. 朝日新聞 $「$ 二億八千万円自民は減額」朝日新聞 2000 年 6 月 28 日、 p. 4 .

(Asahi Shinbun: 280 Millionen Yen: Einbußen für die LDP.)

Asahi Shinbun: Kôbo, yobisen, ze-ka hi-ka, in: Asahi Shinbun, 08.07.2003, S. 4.

朝日新聞「公募・予備選・是か非か」朝日新聞 2003 年 7 月 8 日、 p. 4 。

(Asahi Shinbun: Öffentliche Ausschreibung, Vorwahl: ja oder nein?) 
Asahi Shinbun: Hashimoto gen-shushô, intai mitooshi, in: Asashi Shinbun, 12.08.2005, S. 1. 朝日新聞「橋本元首相、引退見通し」朝日新聞 2005 年 8 月 12 日、 p. 1 .

(Asahi Shinbun: Rücktritt des ehemaligen Ministerpräsidenten Hashimoto absehbar.)

Asahi Shinbun: Yotô 327, shûin-no 2/3 okosu, in: Asahi Shinbun, 12.09.2005, S. 1.

朝日新聞 「与党 327 、衆院の $2 / 3$ 起す」朝日新聞 2005 年 9 月 12 日、 p. 1 .

(Asahi Shinbun: 327 [Mandate] für die Regierungsparteien - 2/3-Mehrheit im Unterhaus .)

Asahi Shinbun: Kôbo. Kihara-shi, sessen seisu, in: Asahi Shinbun, 12.09.2005, S. 1.

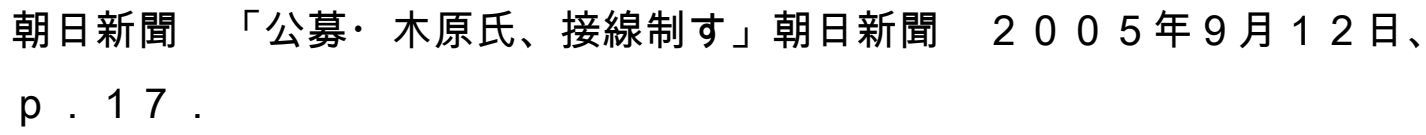

(Asahi Shinbun: Öffentliche Ausschreibung: Kihara kann Kopf-an-Kopf-Rennen für sich entscheiden .)

Der Spiegel: Abgang des Saubermanns, in: Der Spiegel, Nr. 15/1994, S. 138-140.

Der Spiegel: Ende der Verlässlichkeit, in: Der Spiegel, Nr. 26/1993, S. 138-139.

Der Spiegel: Politische Kopfgeburt, in: Der Spiegel, Nr. 49/1994, S. 156.

Der Spiegel: Revolution von oben, in: Der Spiegel, Nr. 30/1993, S. 116-117.

Der Spiegel: Tiefes Bedauern, in: Der Spiegel, Nr. 28/1993, S. 123-124.

Do Rosario, Louise: Father's shoes. Political success becomes a matter of heredity, in: Far Eastern Economic Review, 22.7.1993, S. 16.

Japan aktuell: Gerangel in der LDP um Parlamentskandidaturmodi geht weiter, in: Japan aktuell. Wirtschaft. Politik. Gesellschaft, Heft April/2000, S. 147-158.

Japan aktuell: Gerangel in der LDP um Parlamentskandidatenmode geht weiter, in: Japan aktuell. Wirtschaft. Politik. Gesellschaft, Heft April/2003, S. 130-131.

Japan aktuell: Die Unterhauswahl vom November 2003 im Überblick, in: Japan aktuell. Wirtschaft. Politik. Gesellschaft, Heft Dezember/2003, S. 530-531.

Japan aktuell: Geplante institutionelle Veränderungen in der LDP: Entscheidungsprozesse und Kandidatenauswahl, in: Japan aktuell. Wirtschaft. Politik. Gesellschaft, Heft Februar/2004, S. 33-34.

Japan aktuell: Durchschnittsvermögen von Unterhausabgeordneten niedriger als zuvor, in: Japan aktuell. Wirtschaft. Politik. Gesellschaft, Heft Juni/2004, S. 213.

Japan aktuell: Einkommen von Parlamentariern sinkt 2003 auf neuen Tiefstand, in: Japan aktuell. Wirtschaft. Politik. Heft August/2004, S. 289.

Japan aktuell: Abe als Parteireformer in der LDP? In: Japan aktuell. Wirtschaft. Politik. Ge- 
sellschaft, Heft Oktober/2004, S. 379-380.

Mainichi Shinbun: Jibanmeguri giin honsô, in: Mainichi Shinbun, 14.7.2002, S. 3.

毎日新聞 「地盤めぐり議員奔走」毎日新聞 2002 年 7 月 14 日、 $\mathrm{p}$. 3 .

(Mainichi Shinbun: Die Mühen der Abgeordneten, durch den Wahlkreis zu reisen.)

Moroi, Kaoru: Seshûkeiei-no kenkyû, in: Bungeishunjû, Nr. 10/1992, S. 308-313.

諸井薫「世襲経営の研究」ぶんげいしゅんじゅう No.10/1992,

p. $308-313$.

(Moroi, Kaoru: Eine Untersuchung zu „Erbhöfen“.)

Nihon Keizai Shinbun: Jiyû, kaihô shi gôryû, in: Nihon Keizai Shinbun, 24.07.2003, S. 1.

日本経済新聞「自由、解放し合流」日本経済新聞 2003 年 7 月 24

日、 $\mathrm{p} \cdot 1$.

(Nihon Keizai Shinbun: Liberale: Befreiender Zusammenschluss.)

Yoshida, Tsukasa: Abe Shinzô. Kishi Nobosuke no DNA, ima futatabi, in: AERA, 30.12.2002, S. 67-71.

吉田司「安部晋三。岸信介の DNA、いま再び」AERA、2 002 年 12 月 30 日、p.67- 71 .

(Tsukasa, Yoshida: Die DNA von Kishi Nobosuke, jetzt erneut.)

Yomiuri Shinbun: Sedaikôtai-mo seshû nezuyoku, in: Yomiuri Shinbun, 14.06.2000, S. 4.

読売新聞 「世代交代も世襲根強く」読売新聞 朝刊 2000 年 6 月 14 日、p.4.

(Yomiuri Shinbun: Generationswechsel und Erbfolge tief verwurzelt.)

Yomiuri Shinbun: „kokuminshintô“ 5 nin-de kessei, in Yomiuri Shinbun, 18.08.2005, S. 1. 読売新聞 $「 『$ 国民新党』5人で結成」読売新聞 2005 年 8 月 18 日、 p. 1 .

(Yomiuri Shinbun: „Kokuminshintô“ von fünf Personen gegründet.)

Diverse japanische Zeitungsberichterstattung der Jahre 1998-2005: Asahi Shinbun, Japan Times, Mainichi Shinbun, Nihon Keizai Shinbun, Yomiuri Shinbun.

\subsubsection{Internetquellen}

Japan Times: Lawmakers' average income drops to new low, in: The Japan Times Online, http://search.japantimes.co.jp/print/news/nn07-2005/nn20050705a5.htm, Download vom 12.07.2005. 
Johnston, Eric: Family-bred politicians fan out, in: The Japan Times Online, http://www.japantimes.com/cgi-bin/getarticle.pl5?nn20050910f1.htm, Download vom 12.09.2005.

Kyôto Shinbun: Jiban, kanban, kaban - "sesshû” kôho 180 nin-mo, http://www.kyotonp.co.jp/kp/topics/2000jun/09/13.html, Download vom 28.08.2002.

京都新聞「地盤、看板、かばん『世襲』候補 180 人も」 (Kyôto Shinbun: Jiban, kanban, kaban - unter den Kandidaten wieder 180 Erbpolitiker.)

Maeda, Toshi: Dailies helping bureaucrats keep status quo: van Wolferen, in: The Japan Times Online, http://www.japantimes.co.jp/cgi-bin/getarticle/pl5?nn20000623b1.htm, Download vom 02.07.2005.

Matsuda Takaji: Kohôsha jinzainan, in: Mainichi Shinbun, http://www.mainichimsn.co.jp/shakai/wadai/archive/news/2005/05/16/20050516ddm005070043000c.html, Download vom 02.07.2005.

松田喬知「候補者人材難」

(Matsuda Takaji: Qualitätsschwierigkeiten bei den Kandidaten.)

Ogawa, Tatsuyuki: Daigishi-ni kansuru kenkyû, Hyôgo Kyôiku Daigaku Daigakuin Gakuironbun, Heisei 9, http://ww1.tiki.ne.jp/ otatsu/ron/syn,

Download vom 28.08.2002.

小河達之「代議士に関する研究」兵庫教育大学大学院学位論文 平成 9 年.

(Ogawa Tatsuyuki: Eine Studie zu Abgeordneten, Diss. Hyôgo Kyôiku Daigaku, 1998.)

Yomiuri Shinbun: Hashimoto gen-shushô-no kôkei jinan yôritsu, okayama 4 ku-kara shutsuba http://www.yomiuri.co.jp/politics/news/20050812ia24.htm

Download vom 13.08.2005

読売新聞「橋本元首相の後継二男擁立、岡山 4 区から出馬」

(Unterstützung der Kandidatur des jüngeren Sohnes vom ehemaligen Ministerpräsidenten Hashimoto, er startet inOkayama 4.)

Yomiuri Shinbun: Jimin, 1-ji kônin-kôho 170-nin-o hyômei, http://www.yomiuri.co.jp/politics/news/20050813it13.htm, Download vom 13.08.2005.

読売新聞「自民、1 次公認候補 170 人を表明」

(Die Liberaldemokraten stellen die ersten 170 offiziellen Kandidaten vor.)

Yomiuri Shinbun: Jimin-no Yoshida Rokuzaemon-shi, Kamei-ha-o ridatsu, http://www.yomiuri.co.jp/politics/news/20050812ia24.htm

Download vom 13.08.2005 


\section{読売新聞「自民の吉田六左エ門氏、亀井派を離脱」}

(Yoshida Rokuzaemon von der LDP verlässt die Kamei-ha.)

Yomiuri Shinbun: Shûinsen-no kôhoyôritsu-rasshu, jimin-ha kenkyû-kôbo, http://www.yomiuri.co.jp/politics/news/20050813it01.htm Download vom 13.08.2005

読売新聞「衆院選の候補擁立ラッシュ、自民は緊急公募」

(Vorstoß bei der Unterstützung der Kandidaten, notwendige öffentliche Ausschreibungen bei der LDP.)

http://www.asahi.com/politics/update/0502/003.html, Download vom 02.05.05.

\subsubsection{Sonstige Dokumente}

Jiyûminshutô: Senkyotaisaku yôkô, heisei 6, 24.11.

自由民主党「選挙対策要綱」平成六年十一月二十四日.

(Liberaldemokratische Partei: Prinzipien für die Wahlen, 24.11.1994.)

Jiyûminshutô: Kôhosha sentei kijun, heisei 6, 24.11.

自由民主党「候補者選定基準」平成六年十一月二十四日.

(Liberaldemokratische Partei: Grundsätze der Kandidatenbestimmung, 24.11.1994.)

Jiyûminshutô: Seitô to senkyo, heisei 14, 8.28.

自由民主党「政党と選挙」平成十四年八月二十八日.

(Liberaldemokratische Partei: Partei und Wahlen, 28.08.2002.) 


\subsubsection{Interviews}

In qualitativen Interviews befragte Abgeordnete:

Funada Hajime

Hamada Yasukazu

Harada Yoshitsugu

Hori Kôsuke

Ishiba Shigeru

Itô Shintarô

Kômura Tadamasa

Kôsaka Kenji

Matsuno Yorihisa

Ôno Yoshinori

Terata Manabu

Usui Hideo

Usui Shôichi

Watanabe Shû

Weitere Interviews:

Ishikawa Akimasa, Sprecher der Wahlkampfzentrale der LDP (senkyo taisaku honbu)

Shiratori Rei, Institute for Political Studies in Japan, IPSJ 


\section{Curriculum Vitae}

\section{Kai-Friedrich Donau}

Wilhelmstraße 35

25336 Elmshorn

E-Mail : k_donau@gmx.de

\section{Persönliche Daten}

geboren am 23.07.1976 in Aurich

Staatsangehörigkeit: deutsch

\section{Bildungsweg}

$1983-1987$

$1987-1996$

$1997-2004$

Januar 2004

2004-2006

Juli 2006
Grundschule Hafenstrasse, Elmshorn

Gymnasium Bismarckschule Elmshorn, mit Abiturprüfung

Studium der Politikwissenschaft, Medien- und Kommunikationswissenschaft, Japanologie an der Georg-AugustUniversität Göttingen

Studienabschluss mit Verleihung des Hochschulgrades Magister Artium (mit Auszeichnung)

Promotionsstudium Politikwissenschaft an der Georg-AugustUniversität Göttingen

Disputation

\section{Studien- und Forschungsaufenthalte im Ausland}

März 2001

$2001-2002$

April 2003 - Juni 2003

Februar 2005 - April 2005
Kyôto University of Foreign Studies (Programm des Japanisch-Deutschen Zentrums Berlin)

Studium an der Universität Chiba/Japan (Stipendium des japanischen Bildungsministeriums)

Forschungsaufenthalt an der Tôkyô University of Foreign Studies (DAAD-Kurzstipendium für Abschlussarbeiten)

Forschungsaufenthalt an der Universität Tôkyô (Doktorandenstipendium des DAAD) 